\title{
Ontology of Designing
}

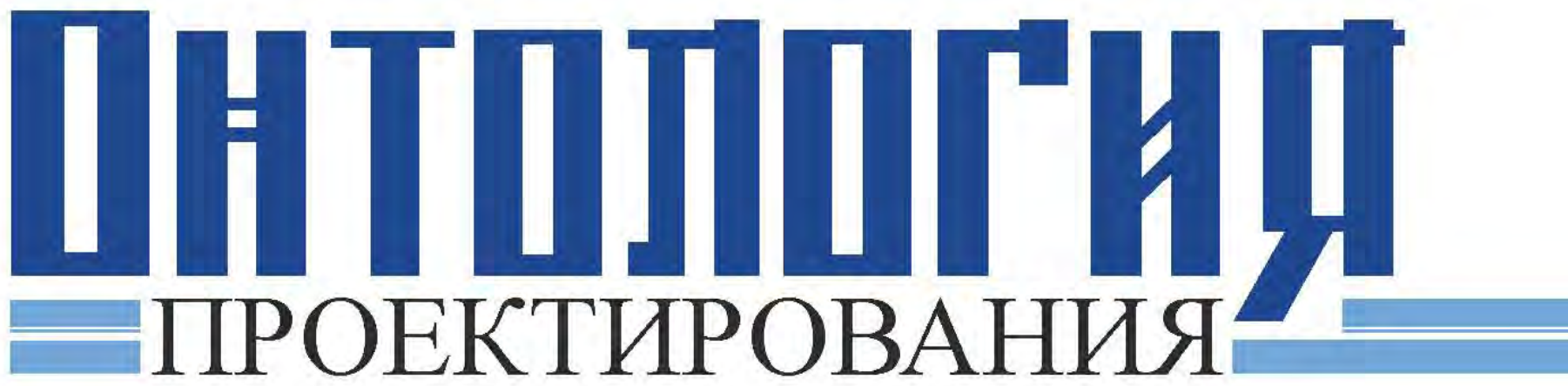




\section{Ontology of Designing}

\section{ㅁТ미ㅁㅐㅐㅐ \\ ПРОЕКТИРОВАНИЯ}

Научный журнал

Том 7

№ 2(24)

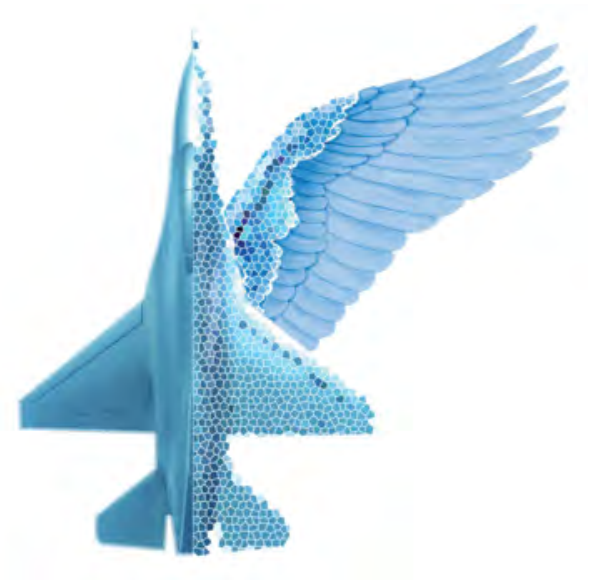


ISSN 2223-9537 (Print)

ISSN 2313-1039 (Online)
«ОНТОЛОГИЯ ПРОЕКТИРОВАНИЯ»

том 7 № 2(24)/2017

DOI:10.18287/2223-9537-2017-7-2

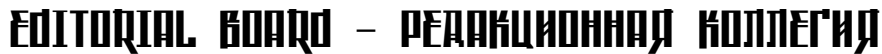

Anatoly I. Belousov Белоусов Анатолий Иванович, д.т.н., профессор, Самарский университет, г. Самара

Nikolay M. Borgest Боргест Николай Михайлович, к.т.н., профессор Самарский университет, член IАОА, г. Самара

Yuri R. Valkman Валькман Юрий Роландович, д.т.н., профессор, МНУЦ ИТиС НАН и МОН Украины, г. Киев

Stanislav N. Vasiliev Васильев Станислав Николаевич, д.т.н., профессор, академик РАН, ИПУ РАН, г. Москва

Vladimir A. Vittikh Виттих Владимир Андреевич, д.т.н., профессор, ИПУСС РАН, г. Самара

Vladimir V. Golenkov Голенков Владимир Васильевич, д.т.н., профессор, БГУИР, г. Минск

Vladimir I. Gorodetsky Городецкий Владимир Иванович, д.т.н., профессор, СПИИРАН, г. Санкт-Петербург

Alexander S. Kleshchev Клещёв Александр Сергеевич, д.ф.-м.н., профессор, ИАПУ ДВО РАН, г. Владивосток

Valery A. Komarov Комаров Валерий Андреевич, д.т.н., профессор, Самарский университет, г. Самара

Sergey M. Krylov Крылов Сергей Михайлович, д.т.н., профессор, СамГТУ, г. Самара

Victor M. Kureichik Курейчик Виктор Михайлович, д.т.н, профессор, Технологический институт ЮФУ, г. Таганрог

Dmitry V. Lande Ландэ Дмитрий Владимирович, д.т.н, с.н.с., ИПРИ НАН Украины, г. Киев

Lyudmila V. Massel Macсель Людмила Васильевна, д.т.н., профессор, ИСЭМ СО РАН, г. Иркутск

Dmitry A. Novikov Новиков Дмитрий Александрович, член-корреспондент РАН, ИПУ РАН, г. Москва

Alexander V. Palagin Палагин Александр Васильевич, д.т.н., проф., академик НАН Украины, Ин-т кибернетики, г. Киев

Semyon A. Piyavsky Пиявский Семён Авраамович, д.т.н., профессор, СамГТУ, г. Самара

Yury M. Reznik Резник Юрий Михайлович, д.филос.н., проф., Институт философии РАН, г. Москва

George Rzevski Ржевский Георгий, профессор, Открытый университет, г. Лондон

Peter O. Skobelev Скобелев Петр Олегович, д.т.н., НПК «Разумные решения», г. Самара

Sergey V. Smirnov Смирнов Сергей Викторович, д.т.н., ИПУСС РАН, г. Самара

Peter I. Sosnin Соснин Петр Иванович, д.т.н., профессор, УлГТУ, г. Ульяновск

Dzhavdet S. Suleymanov Сулейманов Джавдет Шевкетович, академик, вице-президент АН РТ, г. Казань

Robert I. Tuller Таллер Роберт Израилевич, д.филос.н., профессор, Самарский университет, г. Самара

Boris E. Fedunov Федунов Борис Евгеньевич, д.т.н., профессор, ГосНИИ авиационных систем, г. Москва

Altynbek Sharipbay Шарипбай Алтынбек, д.т.н., профессор, Институт искусственного интеллекта, г. Астана

Boris Ya. Shvedin Шведин Борис Яковлевич, к.психол.н., ООО «Дан Роуз», член IAOA, г. Ростов-на-Дону

\section{Executive Editorial Board - Исполнительная редакция}

Chief Editor Smirnov S.V.

Executive Editor Borgest N.M. Editor Kozlov D.M. Editor Krylov S.M.

Technical Editor Simonova A.U. Translation Editor Korovin M.D.
Главный редактор

Выпускающий редактор

Редактор

Редактор

Технический редактор

Редактор перевода
Смирнов С.В.

Боргест Н.М.

Козлов Д.М.

Крылов С.М.

Симонова А.Ю.

Коровин М.Д. зам. директора ИПУСС РАН

директор изд-ва «Новая техника» профессор Самарского университета профессор СамГТУ

редактор изд-ва «Новая техника» аспирант Самарского университета

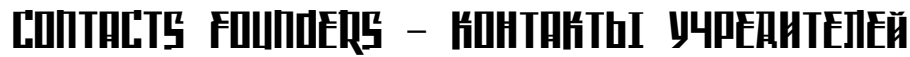

\section{ИПУСС РАН}

443020, Самара, ул. Садовая, 61

тел./факс.: +7 (846) 3332770

\section{Самарский университет}

443086, Самара, Московское шоссе 34, корп. 10, кафедра КиПЛА

тел.: +7 (846) 26746 47, факс.: +7 (846) 2674646
Смирнов С.В.

smirnov@iccs.ru

Боргест H.M.

borgest@yandex.ru

ООО «Новая техника» (издательство)

Адрес редакции: 443010, Самара, ул.Фрунзе, 145, тел.: +7 (846) 33267 84, факс: +7 (846) 3326781

The journal has entered into an electronic licensing relationship with EBSCO Publishing, the world's leading aggregator of full text journals, magazines and eBooks. The full text of JOURNAL can be found in the EBSCOhost ${ }^{\mathrm{TM}}$ databases.

Журнал размещен в коллекции «Издания по естественным наукам» на платформе EastView.

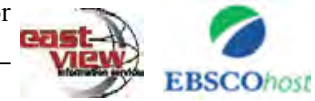

The journal has been successfully evaluated in the evaluation procedure for the ICI Journals Master List 2014, 2015 and journal received the ICV (Index Copernicus Value) of $\mathbf{6 7 . 4 6}$ points (2014), 67.64 (2015).

Журнал включён в перечень рецензируемых научных изданий, в которых должны быть опубликованы основные научные результаты диссертаций на соискание учёной степени кандидата наук, на соискание учёной степени доктора наук (Письмо Департамента аттестации научных и научно-педагогических работников Минобрнауки РФ от 01.12.2015 № 13-6518).

Журнал включён в Российский индекс научного цитирования. Пятилетний импакт-фактор РИНЦ 0.839 (2013 г.), 0.754 (2014 г.), 1.256 (2015 г.). Журнал зарегистрирован в Федеральной службе по надзору в сфере связи, информационных технологий и массовых коммуникаций (Роскомнадзор). Свидетельство ПИ № ФС 77-70157 от 16.06.2017 г.

\section{http://agora.guru.ru/scientific_journal/}

Отпечатано в ООО «Новая техника», г. Самара, пр.К.Маркса, 24-76. Дата выхода 29.06.2017. Тираж 300 экз. Свободная цена. (6+).
(C) Все права принадлежат авторам публикуемых статей (c) Издательство «Новая техника», 2011-2017

(с) Самарский университет, 2015-2017

(с) ИПУСС РАН, 2015-2017 
[DIEP米月HHE

\section{ОТ РЕДАКЦИИ}

ГЕШТАЛЬТ: ГЕНЕЗИС, ПОИСК, ПРОЕКТИРОВАНИЕ

121-122

\section{ПРИКЛАДНЫЕ ОНТОЛОГИИ ПРОЕКТИРОВАНИЯ}

В.В. Голенков, В.В. Таберко, Д.С. Иванюк, К.В. Русецкий, Д.В. Шункевич,

И.Т. Давыденко, В.В. Захаров, В.П. Ивашенко, Д.Н. Корончик

123-144 ПРОЕКТИРОВАНИЕ ПРЕДПРИЯТИЙ

РЕЦЕПТУРНОГО ПРОИЗВОДСТВА НА ОСНОВЕ ОНТОЛОГИЙ

С.В. Лебедев, Н.А. Жукова

СЛИЯНИЕ МЕДИЦИНСКИХ ДАННЫХ НА ОСНОВЕ ОНТОЛОГИЙ

А.М. Беглер

160-171

ИНФОРМАЦИОННАЯ МОДЕЛЬ РЕЗУЛЬТАТОВ НАУЧНОГО ИССЛЕДОВАНИЯ

\section{МЕТОДЫ И ТЕХНОЛОГИИ ПРИНЯТИЯ РЕШЕНИЙ}

Э.Я. Рапопорт, Ю.Э. Плешивцева

172-190

ТЕХНОЛОГИЯ ОПТИМАЛЬНОГО ПРОЕКТИРОВАНИЯ

СЛОЖНОСТРУКТУРИРОВАННЫХ СИСТЕМ

С РАСПРЕДЕЛЁННЫМИ ПАРАМЕТРАМИ:

ПРОГРАММНЫЕ СТРАТЕГИИ ПРИНЯТИЯ РЕШЕНИЙ

В.А. Комаров

191-206

ПРОЕКТИРОВАНИЕ СИЛОВЫХ АДДИТИВНЫХ КОНСТРУКЦИЙ:

ТЕОРЕТИЧЕСКИЕ ОСНОВЫ

\section{ИНЖИНИРИНГ ОНТОЛОГИЙ}

Г.С. Розенберг

ОЦЕНКА ДЛИНЫ ОБУЧАЮЩЕЙ ПОСЛЕДОВАТЕЛЬНОСТИ

В ЗАДАЧЕ РАСПОЗНАВАНИЯ ОБРАЗОВ (БИОИНДИКАЦИЯ)

П.С. Шильников

216-226

ОНТОЛОГИИ УПРАВЛЕНИЯ КАЧЕСТВОМ

КОМПЬЮТЕРНЫХ ДАННЫХ ОБ ИЗДЕЛИИ

Коммюнике Онтологического Саммита 2017:

ИИ, МАШИННОЕ ОБУЧЕНИЕ, ЛОГИЧЕСКИЙ ВЫВОД И ОНТОЛОГИИ

(перевод на русский)

Научные конференции 2017

239-240 


\section{FROM THE EDITORS}

GESTALT: GENESIS, SEARCH, DESIGNING

\section{APPLIED ONTOLOGY OF DESIGNING}

V.V.Golenkov, V.V.Taberko, D.S. Ivanyuk, K.V. Rusetski, D.V. Shunkevich,

I.T. Davydenko, V.V. Zakharov, V.P. Ivashenko, D.N. Koronchik

S.V. Lebedev, N.A. Zhukova

E.Ya. Rapoport, Yu.E. Pleshivtseva TECHNOLOGY OF OPTIMAL DESIGNING OF COMPLEX STRUCTURED SYSTEMS WITH DISTRIBUTED PARAMETERS: PROGRAM STRATEGY OF DECISION MAKING

V.A. Komarov THEORETICAL BASIS FOR DESIGN OF LOAD-BEARING STRUCTURES PRODUCED USING ADDITIVE TECHNOLOGIES

\section{ONTOLOGY ENGINEERING}

\section{G.S. Rozenberg}

THE ESTIMATE OF THE LENGTH OF THE TRAINING SEQUENCE

IN THE TASK OF PATTERN RECOGNITION (BIOINDICATION)

P.S. Shilnikov

ONTOLOGIES OF PRODUCT DATA QUALITY MANAGEMENT

ONTOLOGY SUMMIT 2017 COMMUNIQU'E AI, LEARNING, REASONING AND ONTOLOGIES

(Translation into Russian) 


\section{PERПก́แн月}

\section{ГЕШТАЛЬТ: ГЕНЕЗИС, ПОИСК, ПРОЕКТИРОВАНИЕ GESTALT: GENESIS , SEARCH, DESIGNING} «ештальт - антипод структуры... человек мыслит
гештальтами, а формализовать можно только структуры» ${ }^{1}$

\section{Дорогой наш читатель, уважаемые авторы и члены редакционной коллегии!}

Сформировавшийся образ нашей традиционной рубрики «От редакции» сподвигнул нас обратиться к понятию гештальт. Философский словарь толкует гештальт (нем. Gestalt форма, образ, структура) как пространственно-наглядную форму воспринимаемых предметов, а в переносном смысле употребляется также по отношению к психическим и иным образованиям². Высокоразвитые структуры сознания имеют тенденцию к завершённости, единству, простоте, правильности, к симметрии, к чёткости. Сформировавшиеся гештальты всегда являются целостностями. Ограничение, являющееся основой гештальта, характеризуется степенью резкости и замкнутостью очертаний «контура» образа. При этом каждый гештальт имеет свой «центр тяжести», свой «фокус». О фокусе или сформировавшемся образе олтологии проектирования как научно-образовательной дисциплины рассуждает профессор Самарского университета Н.М. Боргест в своей статье в первом номере журнала за 2017 год «Границы онтологии проектирования», на которую мы ждём отклики от наших читателей.

Актуальной темой для одного из учредителей нашего журнала, Самарского университета, является не столько поиск, сколько проектирование образа униберситета на ближайшую перспективу ${ }^{3}$. Озабоченность этой проблематикой обусловлена амбициозными планами руководства в скорейшем его развитии и прошедшими структурными преобразованиями. Стратегическая цель Самарского университета - войти в число ведущих мировых научнообразовательных центров и создать среду, в которой вырастают высокопрофессиональные специалисты, исследователи, конструкторы, инноваторы, инженеры, востребованные широким спектром отраслей экономики. Одна из стратегических инициатив - продвижение Самарского университета как ведущего в мире университета бысоких технологий, а ближайшая цель коммуникационной стратегии на 2017-2018 годы - формирование образа университета в качестве одного из ведуиих технических вузов России, узнаваемого и признаваемого международным сообществом ${ }^{4}$ В ближайшее время планируется провести стратегические сессии, по итогам которых будет определена целевая модель Самарского университета на ближайшие 15-25 лет и разработаны мероприятия по её реализации ${ }^{5}$. Желаемый гештальт, формируемый усилиями самарских учёных, в первую очередь, в сознании самих «стейкхолдеров», а затем и всех потенциальных «оценщиков» и «заинтересантов» - ближайшая цель и средство продвижения университета в мировое образовательное и научное пространство.

\footnotetext{
' Валькман 1О,Р. Гештальты и метафоры в когнитивной семнотике. Междунар. научн. конф. «Ингеллектуальный анализ информациим, КІІИ, Киев, 2015. - с.31-39.

${ }_{2}^{2}$ Философский энциклопедический словарь. 2010. - http://dic.academic ru/dic.nsf/enc_philosophy/2073/ГЕIITAЛЬТ

${ }^{3}$ На тему будущего университета читайте статьи в нашем журнале «Будущее университета: онтологический подход. В 3 -х частях». Часть I - № 1(2), 2011; Часть 2 - № 1(3), 2012; Часть 3 - № 1(11), 2014.

4 План мероприятий по реализации программы повьшения конкурентоспособности («дорожная карта») федерального государственного автономного образовательного учреждения выспего образования «Самарский нагиональный исследовательский университет имени академика С. П. Королёвал (Самарский университет) на 2013-2()20 годы (3 этап - 2017 год).

${ }^{5}$ Каким Самарский университет будет нерез 15 лет? - http://www.ssau.ru/news/14082/.
} 
На прошедшем в мае Петербургском международном экономическом форуме Глава государства указал общий вектор развития университетов - поддержка передовых научных школ и цифровизация всей страны! Сюда же он отнес и тематику, близкую Самарскому университету: «... обработка и анализ больших массивов данных, искусственный интеллект...» и ряд других. Президент отметил, что «...в авиации и в других сферах, которые являются критически важными для развития России, нужны свои разработки и компетенции. Надо их восстанавливать или создавать заново ${ }^{6}$ ». Сформированный ранее гештальт Самарского университета, основной его аэрокосмический профиль как никогда вновь востребован. Для будущего развития необходимо на «.. .базе ведущих вузов начать формирование инновационных научно-технологических центров, где на одной территории будут сосредоточены и образование, и исследовательская база, и высокотехнологичные, и венчурные компании...». И здесь в Самарском регионе совпадение понимания в векторе развития с Президентом, где важнейшим проектом является создание научно-образовательного и технико-внедренческого комплекса «Гагарин-центр» во главе с якорным университетом.

Гештальты - это тема, которую активно разрабатывал член редколлегии нашего журнала Юрий Роландович Валькман, доктор технических наук, заведующий отделом распределённых интеллектуальных систем Международного научно-учебного центра информационных технологий и систем НАН Украины и МОН Украины, профессор кафедры математических методов системного анализа «Института прикладного системного анализа» Киевского политехнического института им. Игоря Сикорского (КПИ). Он всегда был инициатором и сторонником тесного сотрудничества между российскими и украинскими учёными, интересовался новыми областями искусственного интеллекта и был одним из пионеров таких науч-

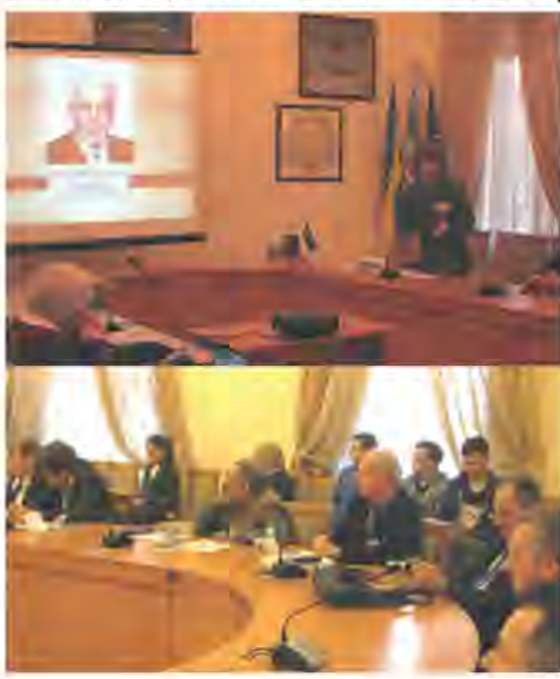
ных направлений как моделирование образного мышления и когнитивная семиотика ${ }^{7}$, готовил обстоятельную статью о гештальтах в наш журнал. Но 16 апреля его не стало...

В мае в КПИ очередная Международная конференция «Интеллектуальный анализ информации-2017» прошла уже без его участия. Ю.Р. Валькман был постоянным членом Программного комитета этой конференции, выступал с пленарными докладами. На открытии конференции с тёплыми словами выступили организаторы, друзья и коллеги Юрия Роландовича - O.P. Чертов, В.С. Степашко, Л.А. Святогор, В.В. Голенков, Н.М. Боргест ${ }^{8}$. Образ, который навсегда останется в нашей памяти о Юрии Роландовиче, - это образ известного учёного, замечательного педагога, доброго и светлого человека ${ }^{9}$.

\section{Уважсаемый автор!}

Мы по-прежнему озабочены формируемым образом нашего журнала, его целостностью, качеством публикуемых работ, стремимся к тому, чтобы его гештальт в сознании наших читателей, авторов, экспертов, научной общественности, в целом соответствовал нашим честным и добрым намерениям. Поэтому мы ждём новых результатов в области компьютерного моделирования знаний, в создании интеллектуальных систем, в онтологии проектирования!

\section{Ontologists and designers of all countries and subject areas, join us!}

\footnotetext{
${ }^{6}$ Путин: Россия ускорит внедрение шифровых технологий - http://www:vestifinance.ru/articles/86338

${ }^{7}$ Валькман Ю.Р. Когнитивная семиотика: гештальты, структуры, целостность, процессы. Междунар. научн. конф. «Интеллектуальный анализ информации〉, КІІ, Киев, 2016, - с.15-24.

${ }^{8}$ Интеллектуалыный анализ информации-2017 - http://pma.fpm.kpi.ua/ru/node/1259

${ }^{9}$ Памяти Юрия Роландовича Валькмана - http://www.raai.org/news/arch_news/2017/04/Valkman_necrolog.pdf
} 


\title{
ПРОЕКТИРОВАНИЕ ПРЕДПРИЯТИЙ РЕЦЕПТУРНОГО ПРОИЗВОДСТВА НА ОСНОВЕ ОНТОЛОГИЙ
}

\author{
В.В. Голенков ${ }^{1}$, В.В. Таберко ${ }^{2}$, Д.С. Иванюк ${ }^{2}$, К.В. Русецкий ${ }^{1}$, Д.В. Шункевич ${ }^{1}$, \\ И.Т. Давыденко ${ }^{1}$, В.В. Захаров ${ }^{1}$, В.П. Ивашенко ${ }^{1}$, Д.Н. Корончик ${ }^{1}$ \\ ${ }^{1}$ Белорусский государственный университет информатики и радиоэлектроники, Минск, Республика Беларусь \\ golen@bsuir.by \\ ${ }^{2} О А О$ «Савушкин продукт», Брест, Республика Беларусь \\ id@pda.savushkin.by
}

\begin{abstract}
Аннотация
В данной работе предлагается подход к проектированию предприятий рецептурного производства на основе формальных онтологий на примере белорусского предприятия ОАО «Савушкин продукт». Рассмотрены вопросы формализации стандартов, на основе которых осуществляется деятельность производства на примере стандарта ISA-88. Предлагается рассматривать стандарт ISA88 как онтологию предметной области рецептурного производства, записанную на естественном языке. Формализация стандарта, таким образом, состоит в отображении структуры и содержания исходного текста документа стандарта на иерархию предметных областей и соответствующих им онтологий. Формальное представление стандарта является основой корпоративной интеллектуальной системы и позволяет автоматизировать решение целого ряда задач, включая информационное обслуживание сотрудников, приведение производства в соответствие стандартам и формальную оценку такого соответствия. Принципиально новым в данной работе является применение в качестве основы для построения интеллектуальной системы предприятия формального представления промышленного стандарта, выполненного на базе графодинамических моделей представления и обработки знаний.
\end{abstract}

Ключевые слова: онтология, онтологическое проектирование, интеллектуальная система, предприятие рецептурного производства, стандарт ISA-88.

Цитирование: Голенков, В.В. Проектирование предприятий рецептурного производства на основе онтологий / В.В. Таберко, Д.С. Иванюк, В.В. Голенков, К.В. Русецкий, Д.В. Шункевич, И.Т. Давыденко, В.В. Захаров, В.П. Ивашенко, Д.Н. Корончик // Онтология проектирования. 2017. - Т. 7, №2(24). - С. 123-144. - DOI: 10.18287/2223-9537-2017-7-2-123-144.

\section{Введение}

Статья посвящена рассмотрению различных аспектов повышения уровня автоматизации предприятий на примере предприятия рецептурного производства ОАО «Савушкин продукт». Актуальность этих исследований обусловлена тем, что без высокого уровня автоматизации и без высоких темпов повышения этого уровня современное предприятие не может добиться высокого уровня конкурентоспособности. Эффективность использования интеллектуальных технологий для повышения уровня автоматизации предприятия, прежде всего, определяется готовностью руководства к развитию предприятия в данном направлении, а также пониманием того, что переход предприятия на новый уровень автоматизации требует фундаментального переосмысления организации его деятельности.

Целью работы является построение онтологической модели предприятия рецептурного производства как средства повышения уровня автоматизации такого рода предприятий и способности адаптации предприятия к постоянно меняющимся внешним условиям. 


\section{1 Проблемы автоматизации предприятий}

Существующие средства автоматизации деятельности предприятия имеют высокую стоимость, трудны в освоении и адаптации к конкретному производству. Как правило, такие средства, с одной стороны, жёстко ориентированы на решение некоторого ограниченного класса задач, с другой стороны, разработчики стремятся сделать такого рода средства как можно более универсальными, наращивая их частными решениями, что приводит к сложности и громоздкости таких систем. Вследствие подобного подхода к наращиванию функционала существующие средства автоматизации деятельности предприятия имеют низкий уровень гибкости (возможности внесения изменений), что приводит к существенным накладным расходам при адаптации таких средств к новым требованиям. Как правило, внесение изменений в указанные средства требует вмешательства разработчиков (часто сторонних с точки зрения предприятия), что влечёт значительные временные и финансовые затраты. Как следствие указанных проблем, далеко не всякое предприятие может обеспечить высокий уровень автоматизации своей деятельности, даже в случае наличия на рынке подходящих решений.

Отсутствие общих унифицированных моделей и средств построения систем автоматизации деятельности предприятия приводит к большому количеству дублирований аналогичных решений как в рамках различных предприятий, так и в рамках разных подразделений одного предприятия. При этом часто возникает ситуация, когда некоторые частные системы, решающие различные задачи в рамках одного предприятия, оказываются несовместимыми между собой, что приводит к дополнительным расходам на реализацию механизмов согласования, например, преобразование форматов данных. Отсутствие такого рода моделей препятствует дальнейшему повышению уровня автоматизации предприятия, в частности, в области автоматизации принятия решений в нештатных ситуациях, прогнозирования дальнейшего развития событий.

\section{2 Требования, предъявляемые к средствам автоматизации предприятий}

Средства автоматизации предприятия должны оперативно и с минимальными затратами времени сотрудников адаптироваться к любым изменениям самого производства - к расширению или сокращению объёмов производства, изменениям номенклатуры производства, изменению используемого оборудования, изменению общей структуры производства, изменению взаимодействия с поставщиками и потребителями, к изменению нормативноправовых актов (включая стандарты), к различного рода непредвиденным обстоятельствам. Адаптация средств автоматизации предприятия ко всем видам изменений самого предприятия и всем аспектам его взаимодействия с внешней средой требует внесения изменений в модель предприятия, полностью отражающую текущее состояние его деятельности.

Средства автоматизации предприятия должны быть гибкими не только для оперативной адаптации к реконфигурации производства, но и для оперативного внесения изменений в сами средства автоматизации в направлении их постоянного совершенствования. Здесь существенным является не только снижение трудоёмкости повышения уровня автоматизации, но и поддержка высоких темпов повышения уровня автоматизации, а также чётко продуманный переходный процесс от одного уровня автоматизации к следующему, в ходе которого одновременно используется и устаревший вариант, и новый.

Эксплуатация системы автоматизации предприятия текущего уровня и перманентный процесс повышения этого уровня требуют согласованного и квалифицированного взаимодействия сотрудников предприятия. Основой такого взаимодействия является хорошо структурированная, достаточно полная и оперативно актуализируемая модель предприятия, отражающая все аспекты текущего состояния структуры и деятельности предприятия, а также 
планы его развития. Такого рода комплексная модель называется знаниями предприятия, которыми надо управлять (добывать, хранить, модернизировать, распространять и т.д.) [1, 2].

Повышение уровня автоматизации предприятия предполагает существенное расширение числа автоматически или автоматизированно решаемых задач, а это, в свою очередь, приводит к автоматизации решения интеллектуальных задач, т.е. к использованию технологий искусственного интеллекта. К числу интеллектуальных задач, решаемых на предприятии, можно отнести:

- анализ производственных ситуаций (в том числе нештатных);

- принятие решений на различных уровнях;

- планирование поведения в сложных обстоятельствах;

- генерация, актуализация документации;

- обучение новых и повышение квалификации действующих сотрудников; и т.д.

Для того, чтобы обеспечить широкое применение технологий искусственного интеллекта в автоматизации предприятия, все корпоративные знания предприятия должны быть записаны на формальном языке представления знаний. При этом указанный язык должен быть удобен не только для использования в интеллектуальных компьютерных системах, но и для использования всеми сотрудниками предприятия.

\section{3 Подходы к автоматизации предприятий}

В настоящее время существует ряд подходов, ориентированных на повышение уровня автоматизации и гибкости предприятий различного рода. Рассмотрим те из них, которые оказали влияние на развитие подхода, предлагаемого в данной работе.

Онтологические модели предприятий. Подход к проектированию различного рода систем на основе онтологических моделей широко используется в настоящее время [3], при этом в особую область исследований выделяют «онтологии предприятия» [2]. Суть предлагаемых подходов состоит в построении онтологий, описывающих деятельность того или иного предприятия или его подразделений. Недостатками данных моделей являются отсутствие унификации представления различных видов знаний предприятия, отсутствие единого подхода к выделению и формированию онтологий, отсутствие единого подхода к построению иерархии онтологий, что ограничивает возможность построения комплексной взаимосвязанной системы онтологий.

Модели управления знаниями предприятий. Управление знаниями в организации - это систематический процесс идентификации, использования и передачи информации, знаний, которые люди могут создавать, совершенствовать и применять. Это процесс, в ходе которого организация генерирует знания, накапливает их и использует в интересах получения конкурентных преимуществ. В настоящее время управление знаниями предприятия реализуется в виде систем управления знаниями [4]. Наиболее актуальным направлением в формализации процесса накопления и управления знаниями предприятия является применение онтологического подхода к построению моделей такого рода процессов.

Модели ситуационного управления. Термин «ситуационное управление» впервые появился в работах Д.А. Поспелова [5]. Это направление получило дальнейшее развитие [6, 7], а в ряде новых работ показано применение в реализации методов ситуационного управления онтологического подхода $[8,9]$. Таким образом, модели и методы ситуационного управления могут быть использованы при построении онтологической модели предприятия с целью повышения эффективности разрабатываемых решений в конкретных производственных ситуациях. 
Многоагентные модели предприятий. В настоящее время многоагентная модель широко применяется при проектировании систем автоматизации производства на различных уровнях. Удобство такого подхода и широта его использования обусловлены схожестью многоагентной модели с реальными процессами, происходящими на предприятии. Действительно, в классической многоагентной системе под агентом понимается некий субъект, как правило, активный и способный взаимодействовать с окружающей средой [10, 11]. Будучи объединёнными в коллективы, такие агенты способны решать задачи гораздо более сложные, чем мог бы решить один агент. К достоинствам многоагентного подхода можно отнести возможность построения на его основе распределённых многоуровневых систем.

Наиболее очевидной интерпретацией такого рода модели в применении к конкретному предприятию является рассмотрение его работников как агентов, каждый из которых способен решать определённый класс задач, вынужденных координировать свои действия для достижения общей коллективной цели. С учётом иерархии структурных подразделений конкретной организации могут быть выделены и уровни иерархии агентов, соответствующие отделам или цехам.

Модели реинжиниринга бизнес-процессов предприятий. Реинжиниринг бизнеспроцессов - это фундаментальное переосмысление и радикальное перепроектирование бизнес-процессов предприятий для достижения резких, скачкообразных улучшений в основных актуальных показателях их деятельности: стоимость, качество, услуги и темпы [12]. Он базируется на понятиях будущего образа фирмы и модели бизнеса, раскрываемых в [13]. Для того, чтобы повысить эффективность реинжиниринга, необходимо обеспечить возможность построения формальных моделей, описывающих предприятие на разных уровнях детализации, и обеспечить унификацию таких моделей, их интегрируемость и иерархичность.

Основной недостаток всех приведённых выше моделей заключается в том, что ни одна из них не обладает достаточной полнотой, и для наиболее адекватного соответствия реальному предприятию его модель должна быть результатом интеграции всех этих моделей.

\section{4 Предлагаемый подход к автоматизации предприятий}

В основе предлагаемого подхода к решению указанных проблем лежат следующие принципы.

- Предприятие рассматривается как распределённая, интеллектуальная социотехническая система, в основе которой лежит хорошо структурированная общая база знаний предприятия.

- В рамках базы знаний предприятия интегрируются все вышеуказанные модели.

- Предприятие рассматривается как иерархическая многоагентная система. В качестве агентов выступают как сотрудники предприятия, так и программные (программноаппаратные) агенты. Иерархичность многоагентной системы означает то, что агенты могут быть неатомарными, т.е. коллективами взаимодействующих между собой агентов, причём такая структура может быть многократно вложенной.

- Весь комплекс средств (как информационных, так и материальных), обеспечивающих деятельность предприятия, оформляется в виде интегрированной распределённой интеллектуальной системы, которую будем называть интеллектуальной корпоративной системой. Основными пользователями этой системы являются сотрудники предприятия.

- Проектирование онтологической модели предприятия сводится к проектированию онтологической модели его интеллектуальной корпоративной системы, которая далее может интерпретироваться имеющимся набором материальных ресурсов. При этом онтологическая модель предприятия является и объектом, и результатом проектирования. 
Для реализации корпоративной системы предприятия предлагается использовать технологию OSTIS $[14,15]$. Из этого следует:

- в качестве основы для представления знаний используется унифицированный, универсальный язык представления - SC-код;

- разработка системы сводится к разработке её модели, описанной средствами SC-кода (sc-модели), которая затем интерпретируется одной из платформ интерпретации;

- база знаний имеет иерархическую структуру, позволяющую рассматривать хранимые знания на различных уровнях детализации (прежде всего это иерархия предметных областей (ПрО) и соответствующих им онтологий [16]);

- частью технологии являются средства коллективного проектирования баз знаний, средства проектирования машин обработки знаний и их компонентов;

- модель обработки знаний основана на многоагентном подходе, позволяющем строить параллельные асинхронные машины обработки знаний, интегрировать различные частные модели обработки в рамках одной системы;

- все агенты взаимодействуют исключительно посредством общей памяти, хранящей конструкции SC-кода (sc-памяти); такой подход позволяет обеспечить гибкость системы и возможность параллельного решения различных задач [17];

- для разработки программ агентов используется внутренний параллельный язык SCP, тексты которого также представлены в SC-коде, что позволяет обеспечить платформенную независимость таких агентов.

На данном этапе работы основное внимание уделено решению задачи разработки онтологической модели базы знаний, в частности - построению набора онтологий ПрО, описывающих содержание основных стандартов. Формальное представление стандартов является основой для согласования всех ключевых аспектов деятельности предприятия и построения общей онтологической модели всего предприятия в целом и отдельных его компонентов.

Чтобы убедиться в актуальности решаемых задач, рассмотрим текущее состояние и историю развития автоматизации на конкретном предприятии.

\section{5 Анализ развития средств автоматизации производства предприятия ОАО «Савушкин продукт»}

В конце 90-х на предприятии было принято решение разрабатывать своими силами платформу системы, которая в дальнейшем позволяла бы реализовать не только промышленные проекты автоматизированных систем управления технологическими процессами (АСУТП), но и решать бухгалтерские, складские и т.п. задачи. В разрабатываемой SCADA-системе (Supervisory Control And Data Acquisition. - диспетчерское управление и сбор данных), названной EasyServer, первым был реализован проект по контролю температур в технологических ёмкостях (танках) аппаратного цеха. После его успешного запуска и получения подтверждения эффективности принятых решений, были реализованы проекты по автоматизации моечной станции, цеха приёмки молока, цеха сгущения.

В ходе развития системы уровень автоматизации постоянно повышался. На начальном этапе охватывался только сбор и протоколирование данных с датчиков (температура, давление и т.д.). На последующем этапе появился уровень управления технологическими операциями и техническими устройствами. В настоящее время реализуется уровень рецептурного (партионного) производства.

Для разработки проектов также используется среда разработки CODESYS от компании 3S-Smart Software Solutions для контроллеров WAGO. Она бесплатна, позволяет использовать инженерные языки для программируемых логических контроллеров (IEC 61131-3) - IL, 
LD, FBD, SFC, ST. Такой подход используется для разработки относительно простых автономных проектов (например, посты приёмки молока) инженером по автоматизации без привлечения инженера-программиста. Реализованные проекты интегрируются в систему за счёт использования открытого протокола обмена MODBUS TCP.

Для эффективной организации работы цеха требуется решение задач организации взаимодействия между отдельными проектами. Однако нет универсального подхода - в одних проектах используется MODBUS TCP, в других - дополнительный контроллер в качестве коммуникационного шлюза. Кроме того, используются физические соединения для обмена сигналами. Всё это дополнительно усложняет систему.

Использование собственной разработки (как проектов, так и системы в целом) обладает следующими достоинствами.

- Высокая скорость разработки новых проектов. Вся накопленная при разработке функциональность становится частью системы. В настоящее время типовой проект можно разработать силами буквально одного инженера по автоматизации в течение нескольких часов, что позволило реализовать уже более 200 проектов.

- Относительная дешевизна разработанной SCADA-системы EasyServer. Hесмотря на содержание штата квалифицированных разработчиков, затраты на неё оказались гораздо ниже, чем при использовании сторонних решений.

- Обширный функционал собственной системы. Возможности системы сопоставимы с коммерческими аналогами (Simatic Step 7 + Simatic WinCC) за счёт тесной связи с реальным производством.

Существуют следующие недостатки текущего варианта системы автоматизации производства.

- Представление данных реализовано в форме простых отчётов. На этапе запуска первых проектов отчёты были реализованы в виде отдельных приложений на Delphi, данные из таблиц через BDE от сервера проекта экспортировались в MS Excel. На текущий момент принято решение организовать обработку данных (и формирование отчётов в том числе) на более высоком уровне, а на уровне АСУТП оставить только базовые отчёты о работе проектов. Поэтому открыт вопрос о платформе верхнего уровня (уровень предприятия в целом - ERP) для решения отчётных задач.

- Так как приоритетом является скорость разработки и модификации, имеет место низкий уровень документации как системы в целом, так и отдельных проектов. В связи с этим требуются решения, в которых документация становится частью проектируемой системы. В настоящее время описание функциональной схемы автоматизации, электрической части и спецификации проекта реализовано в CAD Eplan. Описание технологической части также выполняется с помощью CAD Eplan, но хранится отдельно в виде сценариев на языке Lua. Описание отдельных устройств (частотные преобразователи, клапаны) хранится в формате PDF и поступает от их производителей.

- Ограниченное время на тестирование и отладку. Поэтому востребованы модули диагностики и самодиагностики для проектов.

- Для успешного освоения рынка необходимо привести организацию производства на предприятии «Савушкин продукт» в соответствие с международными стандартами (в частности, со стандартом ISA-88). Однако стандарты могут являться и сдерживающим фактором - они громоздки, есть сложности с их толкованием, их внедрение может требовать неоправданно больших затрат. Поэтому для реализации процесса приведения предприятия в соответствие с международными стандартами необходимо использовать более гибкую организацию самого производства с учётом эволюции самого стандарта. 
Растёт охват предприятия автоматизацией. Теперь это уже не только отдельные цехи, но и всё предприятие в целом. Поэтому востребованы системы управления не на уровне «оператор» - «мнемокарта техпроцесса» - «отдельный техпроцесс», а «диспетчер производственной логистики» - «интеллектуальный веб-интерфейс» - «завод в целом». Роботизация производства - необходимый элемент развития современного предприятия. Поэтому нужны решения, позволяющие интегрировать и реализовывать новые проекты роботизации производственных процессов и переходу к безлюдному производству.

\section{6 Формализация стандартов рецептурного производства}

\section{1. Принципы фрормализации стандартов}

Основой онтологического подхода к проектированию предприятия является формализация стандартов. Каждый стандарт рассматривается как онтология соответствующей ПрО, являющаяся основой для автоматизированного решения ряда задач, включая информационное обслуживание сотрудников, формальную оценку соответствия предприятия этим стандартам и т.д.

Одной из важных проблем внедрения стандарта на предприятии является возможность неоднозначной трактовки некоторых положений стандарта, а также необходимость постоянной коррекции такой трактовки с целью приближения её к смыслу оригинала. Кроме того, существуют особенности применения стандарта на каждом предприятии, необходимость актуализации используемого стандарта (т.к. любой стандарт постоянно эволюционирует), с последующим внесением изменений в структуру и организацию деятельности предприятия для обеспечения соответствия стандарту.

Одним из путей решения такого рода проблем является построение его формальной семантической модели, которая могла бы одинаково интерпретироваться как компьютерной системой, так и человеком. Формальное семантическое представление стандарта позволяет без внесения каких-либо изменений в структуру такого представления дополнять его различного рода дидактической информацией (примерами, пояснениями и т.д.), которая способствует пониманию стандарта сотрудниками предприятия. Кроме того, формальное семантическое представление стандарта обеспечивает значительное упрощение внесения изменений в такое представление стандарта, которые могут быть вызваны либо уточнением трактовки этого стандарта, либо эволюцией самого стандарта (его исходного документа).

Построение формальной модели стандарта сводится к построению интегрированной формальной онтологии, специфицирующей соответствующую ПрО. Для этого необходимо отобразить структуру и содержание исходного текста стандарта на иерархию ПрО и соответствующих им онтологий.

Использование онтологического подхода позволяет путём добавления интеллектуальных агентов построить на его основе интеллектуальную справочную систему, предоставляющую широкий спектр информационных услуг пользователям, в том числе способную отвечать на широкий спектр вопросов, ответ на которые может быть в явном виде не представлен в тексте стандарта, или поиск его в таком тексте затруднителен.

\section{2. Формализация стандарта ISA-88}

Среди множества стандартов, регулирующих деятельность предприятий, наиболее полно отражает специфику рецептурного производства стандарт ISA-88 [18]. Основным достоинством ISA-88 является разделение ПрО рецептурного производства на максимально независимые ПрО рецептов, оборудования и управления [19]. Такое разделение позволяет специа- 
листам предприятия при решении своих задач осуществлять поиск их решения строго в своей ПрО, независимо от остальных. Этот факт закладывает фундамент в обеспечение гибкости рецептурного производства и сам по себе является основой для построения онтологической модели предприятия.

Стандарт ISA-88 состоит из четырёх частей, однако особую ценность для построения формальной онтологии имеет первая часть, поскольку она предоставляет терминологию и целостный набор понятий и моделей, используемых в управлении рецептурным производством [20]. В статье используются англоязычные термины из [18], русскоязычные термины из ГОСТ Р МЭК 61512-1-2016 [21]. Термины, вновь появившиеся в ревизии ISA-88.00.01 2010 года, в частности, касающиеся процедурной модели, скомбинированы из уже существующих и переведены авторами статьи, исходя из имеющихся официальных переводов.

Структурно исходный документ первой части стандарта ISA-88 состоит из 6 разделов:

1) область применения стандарта (Scope of the standard);

2) нормативные ссылки (Normative References);

3) термины и определения (Definitions);

4) процессы серийного производства и оборудование (Batch Processes and Equipment);

5) понятия, используемые при управлении серийным производством (Batch Control Concepts);

6) действия и функции процесса управления серийным производством (Batch Control Activities and Functions).

Отобразим структуру документа на иерархию ПрО и соответствующих им онтологий. Стандарт ISA-88 в целом соответствует ПрО предприятий рецептурного производства. Первые два раздела специфицируют документ стандарта и непосредственно к описанию ПрО рецептурных производств не относятся и, соответственно, не отображаются на иерархию ПрО и онтологий. Раздел 3 соответствует терминологической онтологии и логической онтологии [16] ПрО предприятий рецептурного производства. Подраздел 4.1 соответствует ПрО процессных моделей рецептурных производств. Остальные подразделы четвёртого раздела соответствуют ПрО физических моделей рецептурных производств. Раздел 5 соответствует ПрО моделей процедурного управления. Раздел 6 соответствует ПрО деятельности по управлению рецептурным производством.

Запишем иерархию ПрО базового уровня в формальном виде на языке SCn [22]:

\section{ПрО предприятий рецептурного производства}

$=>$ частная $\Pi р O^{*}$ :

- ПрО физических моделей рецептурных производств

- ПрО проиессных моделей рецептурных производств

- ПрО моделей прочедурного управления оборудованием рецептурных производств

- ПрО деятельности по управлению рецептурнылм производством

Приведём структурные спецификации [16] частных ПрО на языке SCn.

ПрО физических моделей рецептурных производств

э максимальный класс объектов исследования':

equipment entity

э немаксимальный класс объектов исследования':

- area

= область производства

- site

= место производства

- process cell

= производственный цех

- unit

= технологическая установка

- equipment module 
= блок оборудования

- control module

= блок управления

- enterprise

= препдриятие

- equipment relation

= связь оборудования

ᄏ исследуемое отношение':

содержит *

$=$ contains $*$

\section{ПрО процессных моделей рецептурных производств}

Э максимальный класс объектов исследования':

process element

= процессный элемент

Э немаксимальный класс объектов исследования':

- $\quad$ process stage

= стадия технологического прочесса

- $\quad$ process operation

= технологическая операиия

- process action

= производственное действие

э исследуемое отношение':

process element link*

$=$ связь прочессных элементов*

ПрО моделей процедурного управления оборудованием рецептурных производств

э максимальный класс объектов исследования':

procedural element

= процедурный элемент

э немаксимальный класс объектов исследования':

- $\quad$ process cell procedure

= проиедура производственного иеха

- $\quad$ unit procedure

= процедура технологической установки

- operation

= операция

- $\quad$ phase

= фаза

- $\quad$ recipe procedural element

= проиедурный элемент рецепта

- $\quad$ equipment procedural element

= проиедурный элемент оборудования

- $\quad$ recipe process cell procedure

= рецептурная процедура производственного иеха

- $\quad$ recipe unit procedure

= рецептурная процедура технологической установки

- $\quad$ recipe operation

= рецептурная операчия

- $\quad$ recipe phase

= реиептурная фаза

- $\quad$ equipment process cell procedure

= процедура оборудования производственного ичеха

- $\quad$ equipment unit procedure

= процедура оборудования технологической установки

- equipment operation

= операчия оборудования

- equipment phase 
= фаза оборудования

$\exists$ исследуемое отношение':

execution order*

$=$ порядок исполнения*

Ниже рассмотрены примеры описания физической и процедурной моделей предприятия «Савушкин продукт» на основе системы понятий, исследуемых в соответствующих ПрО.

\section{3. Формализация фризической модели предприятия «Савушкин продукт»}

Предприятие можно специфицировать в различных аспектах, некоторые из которых (процессы, процедуры, оборудование, управление) регулируются стандартом ISA-88. Этим аспектам соответствуют иерархически организованные ПрО. В качестве иллюстрации приведём фрагмент спецификации предприятия «Савушкин продукт» в рамках ПрО физических моделей рецептурных производств. Спецификация имеет семь уровней: enterprise (предприятие), site (место производства), area (область производства), process cell (ячейка процесса), unit (аппарат), equipment module (блок оборудования), control module (блок управления).

\subsection{1. Уровень Enterprise (предприятия)}

Предприятие - самая крупная производственная единица, под которой обычно понимается компания в целом. В нашем случае это ОАО «Савушкин продукт». Формализованная запись этого факта приведена на рисунке 1. Здесь и далее для формализованного представления используется язык SCg [22].

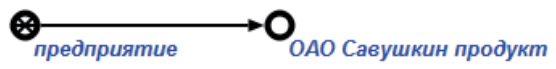

Рисунок 1 - Формализованная запись предприятия «Савушкин продукт»

\subsection{2. Уровень Site (мест производства)}

Предприятие состоит из одного или более мест производств. Места производства чаще всего выделяются по географическому принципу. Места производства, входящие в ОАО «Савушкин продукт», показаны на рисунке 2. Формализованное представление этой информации приведено на рисунке 3. Далее рассматривается место производства «Брест 2».

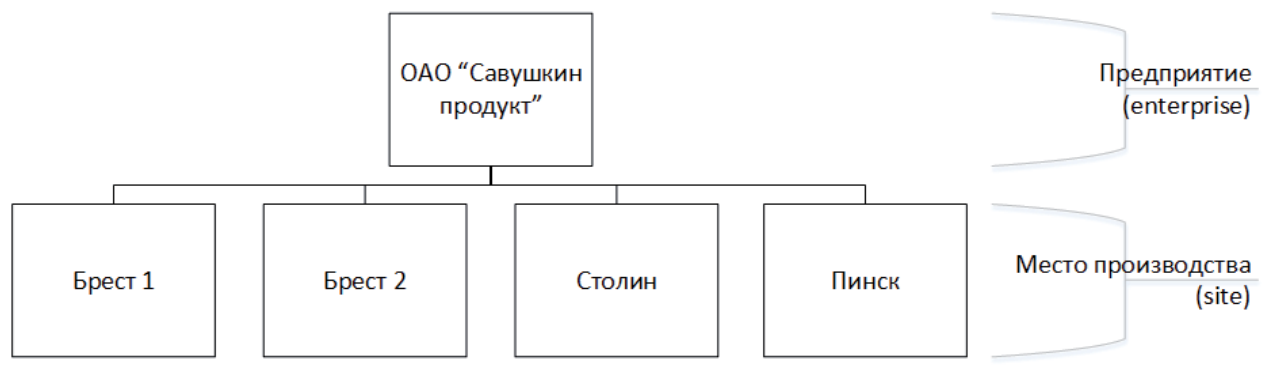

Рисунок 2 - Места производства предприятия «Савушкин продукт»

\subsection{3. Уровень Area (областей производства)}

Место производства состоит из одного или нескольких областей производства. В рамках ISA-88 не все части производства будут являться частью области производства, а только те, которые имеют непосредственное отношение к процессу рецептурного производства. Области производства «Брест 2» приведены на рисунке 4. Формализованное представление данного фрагмента структуры предприятия приведено на рисунке 5. В дальнейшем будет рассматриваться «Цех мягких сыров и творога», выделенный на рисунке 4. 


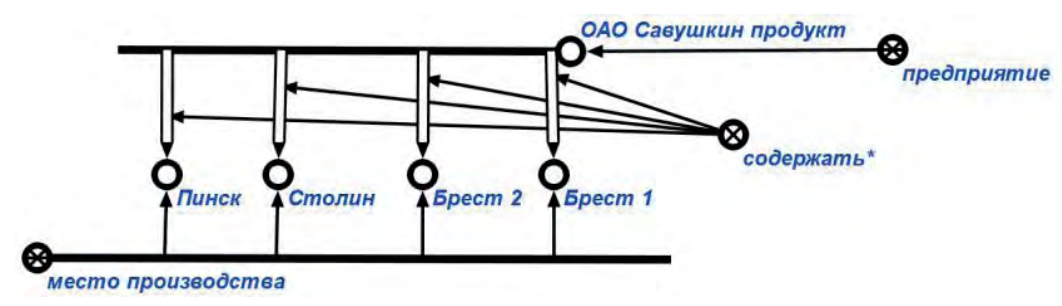

Рисунок 3 - Формализованное представление связи двух верхних уровней физической модели

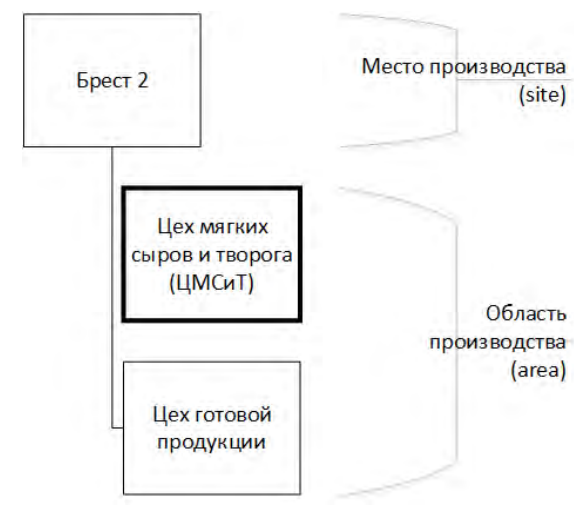

Рисунок 4 - Производственные участки производства «Брест 2»

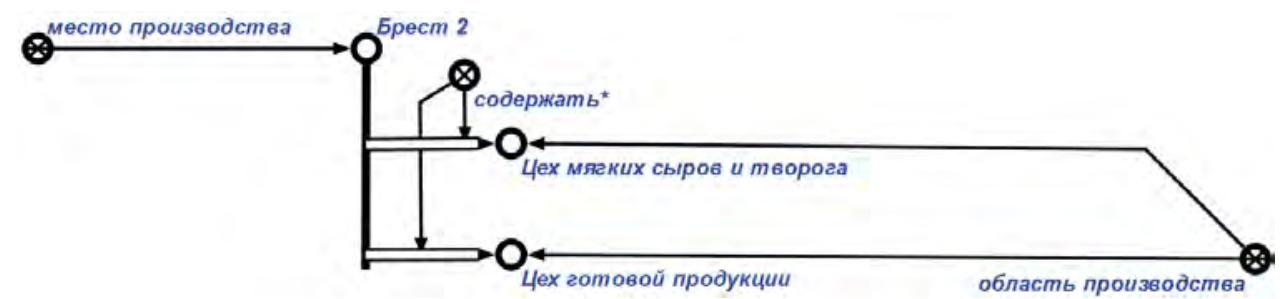

Рисунок 5 - Формализованное представление связи второго и третьего уровней физической модели

\subsection{4. Уровень Process Cell (ячейки процесса)}

Область производства содержит всё оборудование, необходимое для производства партии продукта. Иногда для описания комплекса оборудования производственного участка используется термин «линия» (train). В процессной ячейке может быть более одной линии, последовательность оборудования в которых называется маршрутом (раth). Ячейка процесса получает сырье (raw) или промежуточное вещество (work-in-progress) и преобразует их в готовую продукцию или другое промежуточное вещество. Цех мягких сыров и творога производства «Брест 2» содержит две ячейки процесса - одна отвечает за изготовление творожной массы, а вторая - за её формовку и фасовку (рисунок 6). На рисунке 7 приводится формализованное представление этого фрагмента структуры предприятия. В дальнейшем рассматривается ячейка процесса производства творожной массы «Хуторок» (выделена на рисунке 6).

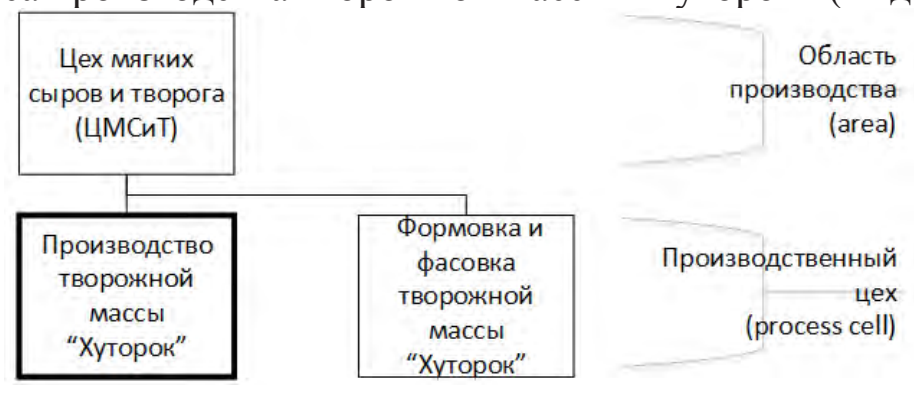

Рисунок 6 - Ячейки процесса в цехе мягких сыров и творога 


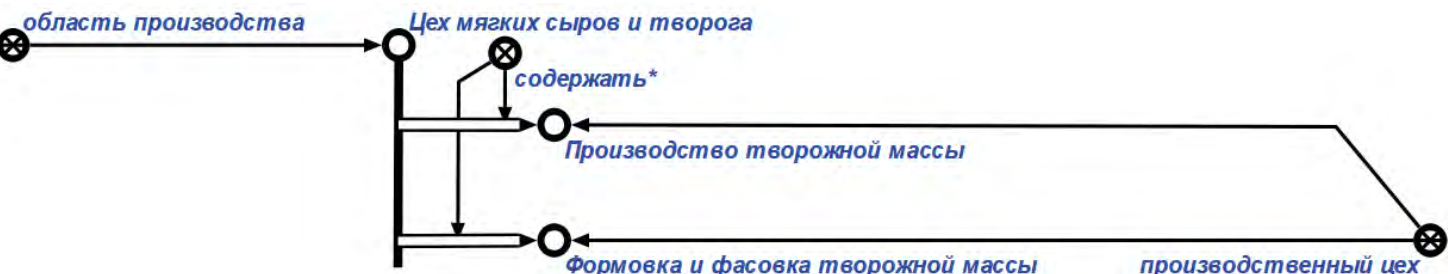

Рисунок 7 - Формализованное представление связи третьего и четвёртого уровня модели

\subsection{5. Нижние уровни фризической модели}

Три нижних уровня модели оборудования рассматриваются совместно, поскольку, согласно ISA-88, и unit, и equipment module, и control module могут непосредственно содержаться в process cell, как показано на рисунке 8.

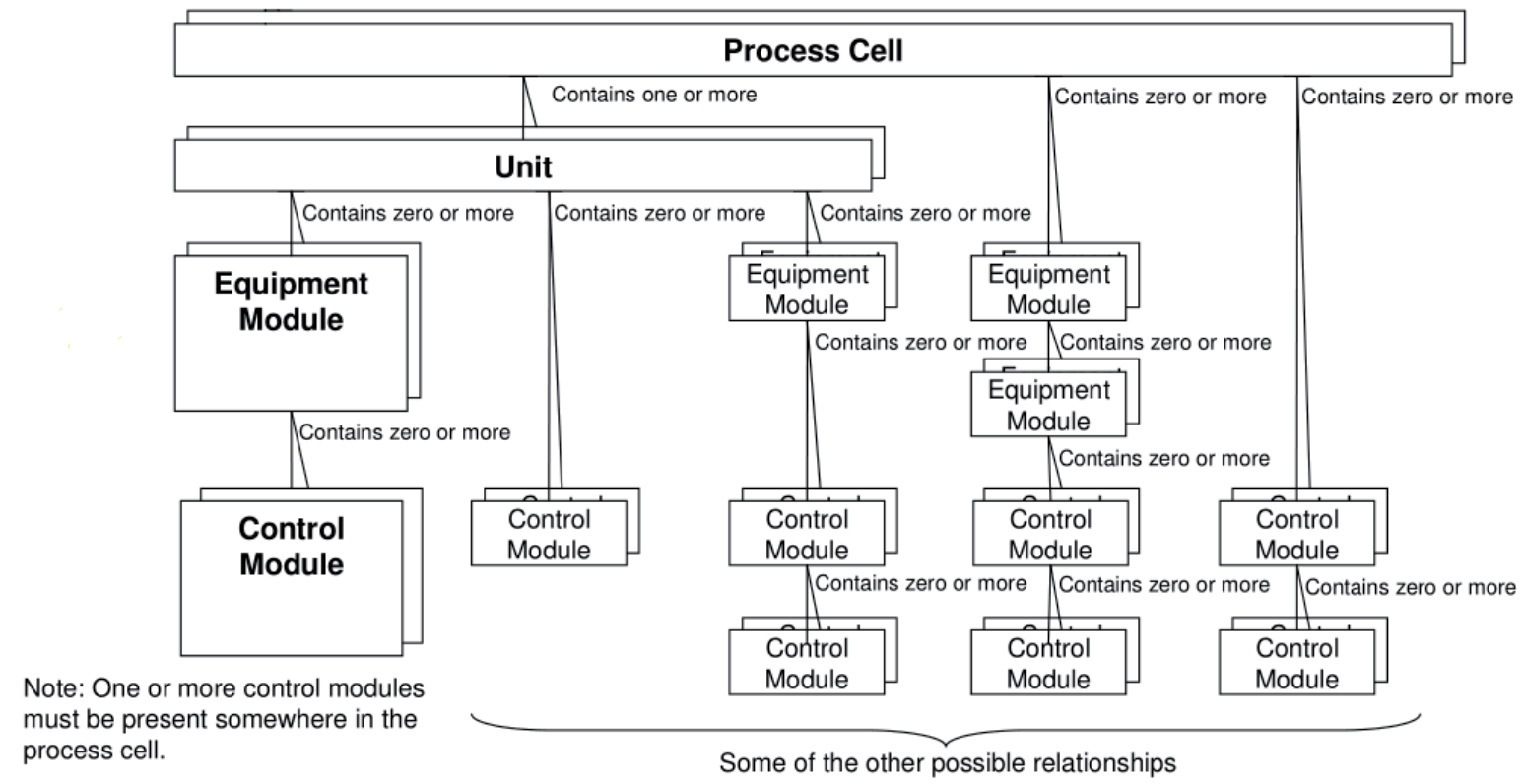

Рисунок 8 - Возможные отношения между сущностями трёх нижних уровней физической модели в ISA-88

Аналогичным образом, аппарат (технологическая установка) может непосредственно содержать как блок оборудования, так и блок управления. Технологическая установка является основной единицей рецептурного производства. Процесс рецептурного производства происходит именно в технологических установках, которые выполняют главные производственные действия (major processing activities), придающие ценность промежуточному или конечному продукту - смешивание, осуществление химических реакций и др.

Блок оборудования представляет собой группу физических устройств, образованную для выполнения некоторой конкретной функции в рамках рецептурного производства. Стандартом ISA-88 подразумевается, что блок оборудования способен реализовывать логику, основанную на принятии решений (decision-based logic). Например, контроль уровня жидкости или давления в некоторой ёмкости или поддержание определённого температурного режима обрабатываемого вещества.

Блок управления - базовый элемент физической модели, представляющий собой связанный набор управляющих модулей (датчики, клапаны и пр.), рассматриваемых как единая сущность. Следует заметить, что не всегда блок управления будет иметь физическую реализацию - он может быть инструкцией или группой инструкций программируемого микроконтроллера или драйвером некоторого устройства. 
В рассматриваемой ячейки процесса по производству творога имеется 32 коагулятора (технологических установок), две линии приёмки смеси, восемь линий выдачи, восемь линий откачки сыворотки и 16 бойлеров (блоков оборудования), что показано на рисунке 9. Формализованное представление нижних уровней физической модели показано на рисунке 10.

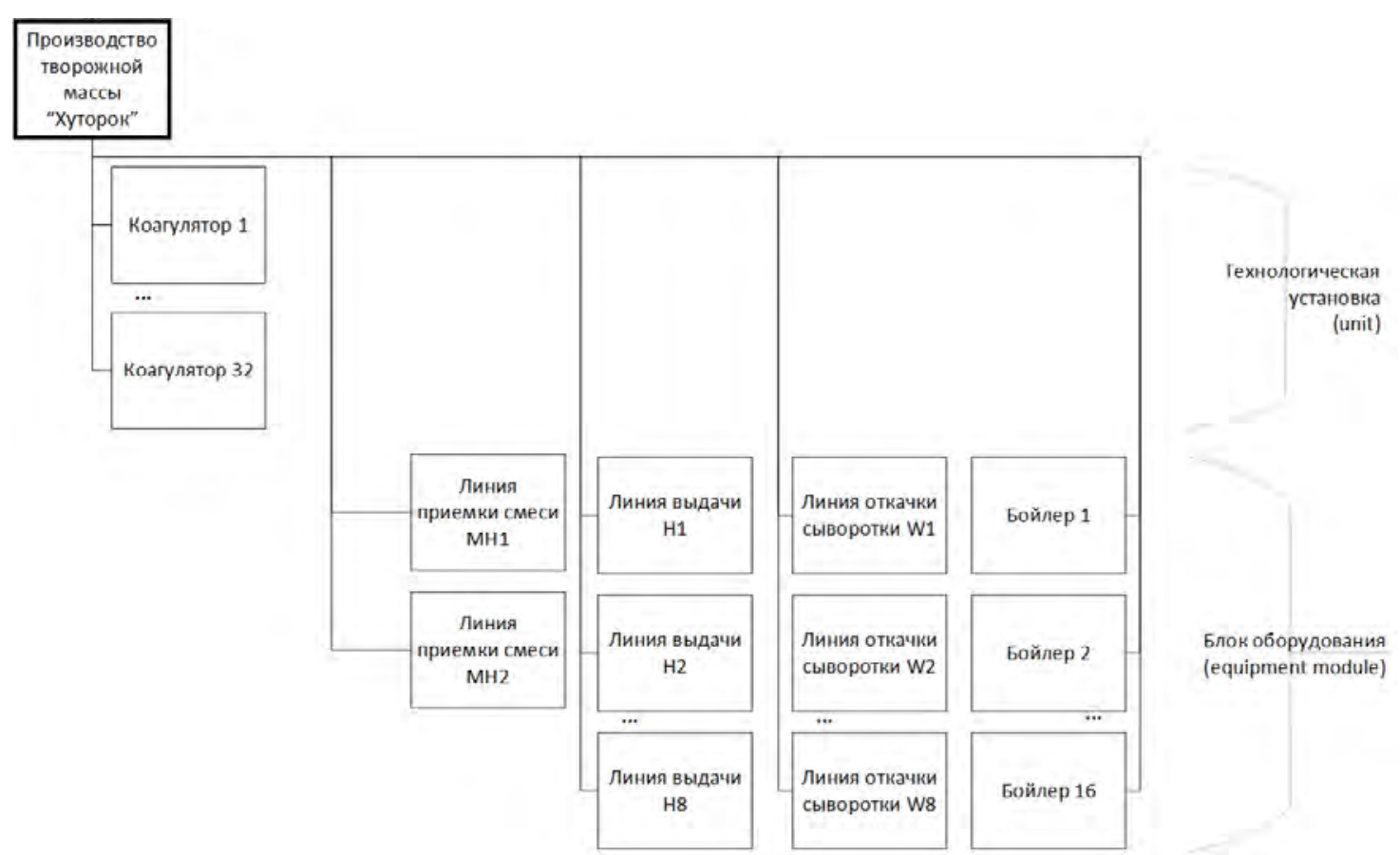

Рисунок 9 - Технологические установки и блоки оборудования в ячейке процесса производства творожной массы
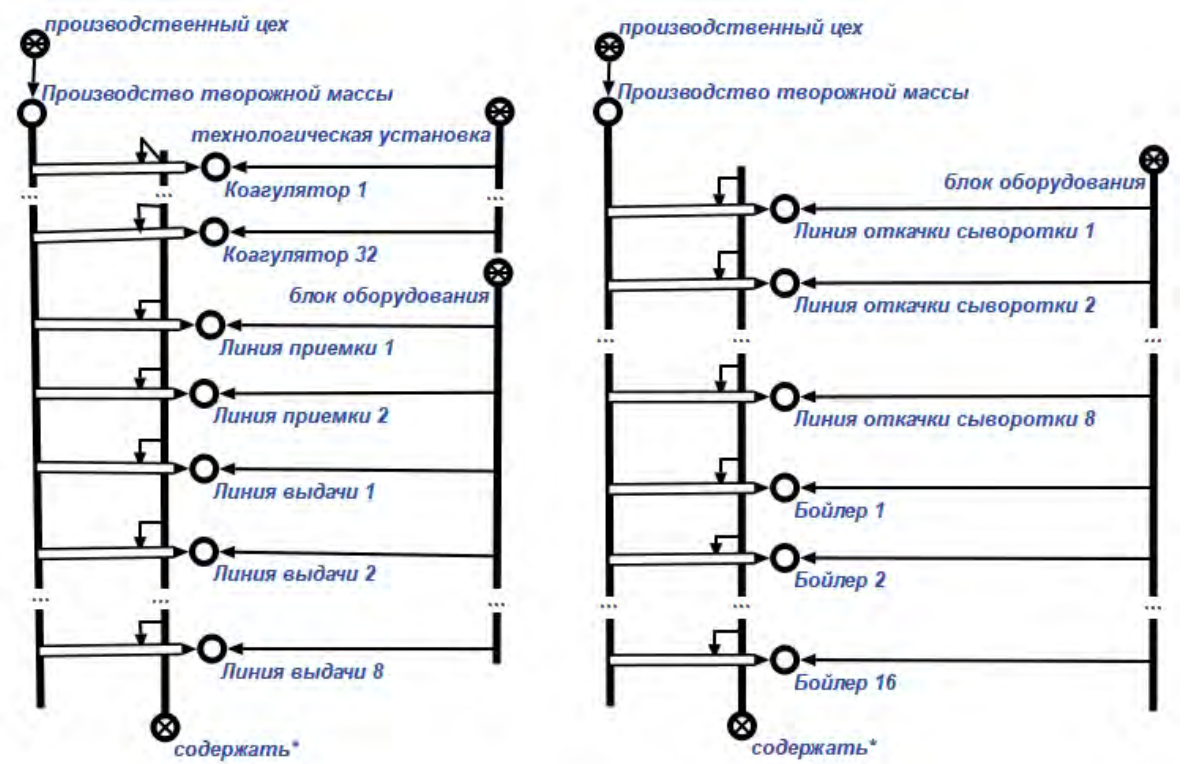

Рисунок 10 - Формализованное представление нижних уровней физической модели

\subsection{6. Формализация технической документации}

Практическое приложение физической модели состоит в формализации документации предприятия, в частности функциональных схем. Принципиальная простота взаимного пере- 
хода между технологическим чертежом и его семантическим представлением даёт возможность обращаться к элементам чертежа, инициировать команды, воздействующие на состояние оборудования, и отслеживать его динамику. Эта функциональность обеспечивается рецепторными и эффекторными агентами, работающими над семантическим представлением документации, хранящимся в общей семантической памяти. Это позволяет «оживить» техническую документацию предприятия, сделать её многоцелевой.

В качестве примера практического приложения рассмотрим изображенный на рисунке 11 в виде функциональной схемы [23] фрагмент ячейки процесса на этапе нагрева творожной массы, использующий два коагулятора KOAG14 и KOAG15 и бойлер В1. Кроме того, на ней изображён ряд датчиков (температурный - TE4, уровня - LS41, LS42), насосов (N4) и клапанов (V40, V41, 14V1, 15V1), а также теплообменник (W1), которые, в соответствии со стандартом ISA-88, относятся к модулям управления, поскольку являются базовыми элементами оборудования, выполняющими атомарные функции. Стрелками показана циркуляция греющей воды по системе подогрева.

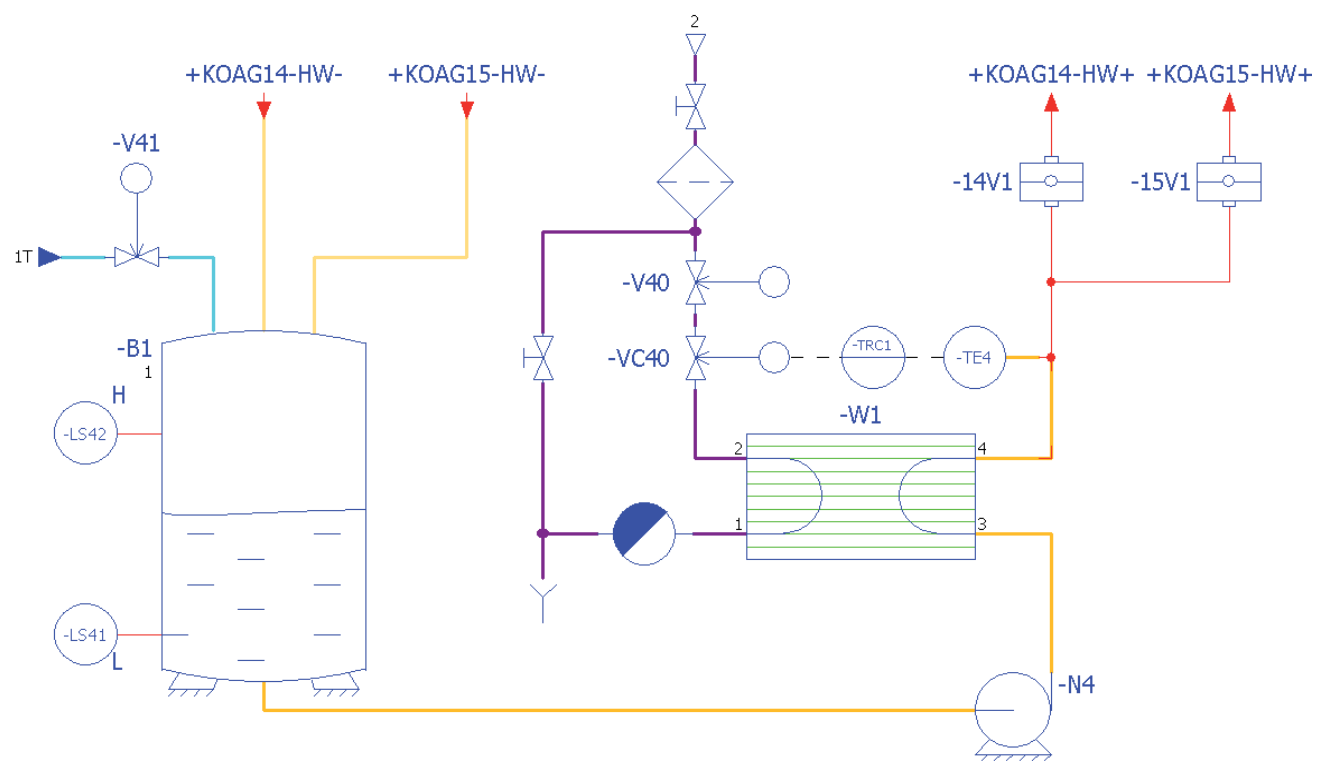

Рисунок 11 - Функциональная схема фрагмента ячейки процесса, выполненная средствами ГОСТ 21.404-85

Рассмотрим процесс перевода технологической схемы в её семантическое представление. Каждой единице оборудования - конкретному датчику, насосу, трубе, коагулятору соответствует узел семантической сети. Тот факт, что конкретная единица оборудования является экземпляром того или иного класса оборудования, обозначается связью от узла, обозначающего класс, к узлу оборудования (рисунок 12).

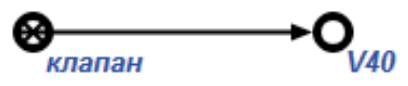

Рисунок 12 - Пример семантического представления экземпляра класса оборудования

Трубы относятся к особому виду оборудования, соединяющему другое производственное оборудование между собой. Если в рамках технологического процесса обрабатываемое вещество по трубе переходит от одной единицы оборудования к другой, то узел единицы оборудования, из которой исходит вещество, связывается со знаком трубы бинарным отношением оборудование-источник*, а узел единицы оборудования, в которое вещество поступает, связывается со знаком трубы отношением оборудование-приемник* (рисунок 13). 


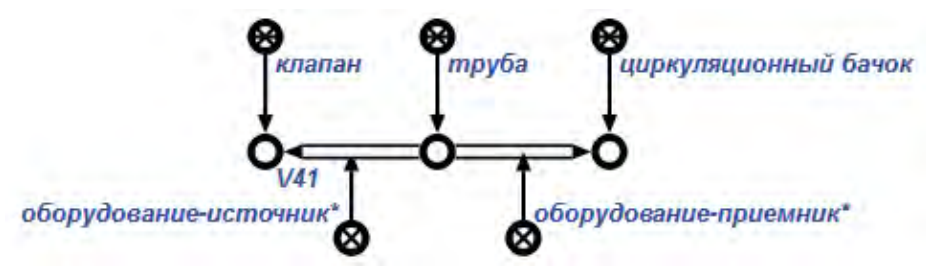

Рисунок 13 - Семантическое представление соединения двух единиц оборудования трубой

Может иметь место односторонняя связь датчика с трубой, непосредственно не относящаяся к перемещению вещества. Она обозначается связкой отношения соединение* между знаком датчика и знаком трубы (рисунок 14).

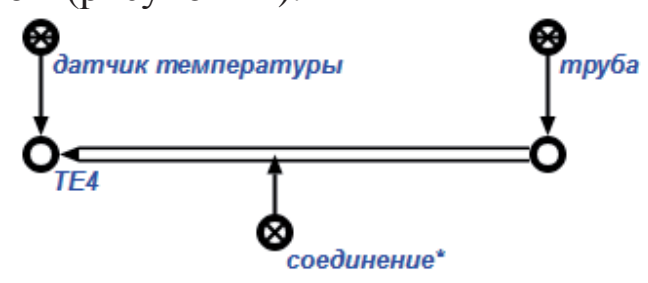

Рисунок 14 - Семантическое представление связи датчика и трубы

На рисунке 11 также представлены в виде отдельных сущностей конструктивные части оборудования (датчики температуры коагуляторов). Для связи единицы оборудования с её конструктивной частью используется связка отношения часть-целое*, связывающая узел единицы оборудования и узел его конструктивной части (рисунок 15).

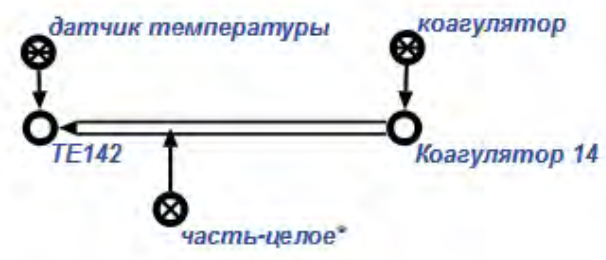

Рисунок 15 - Семантическое представление связи единицы оборудования с его конструктивной частью

Для отдельного цеха полученного описания достаточно. Однако для предприятия с множеством цехов необходима унификация семантического представления на основе общей онтологии. В качестве общей онтологии может выступить онтология ПрО физических моделей рецептурных производств, построенная в результате формализации стандарта ISA-88. Она будет находиться в онтологической модели предприятия уровнем выше онтологии конкретного отраслевого производства, но уровнем ниже онтологии рецептурных производств в целом. Такая иерархическая организация онтологий позволяет обеспечить независимость конкретной производственной рецептуры от конкретного производственного оборудования, поскольку их взаимосвязь будет устанавливаться на более высоком уровне абстракции, что сродни шаблону проектирования «Мост» в объектно-ориентированной парадигме [24].

Чтобы установить связь описания, полученного на первом этапе, с понятиями, определёнными в физической модели, необходимо сопоставить конкретные элементы оборудования с соответствующими уровнями модели оборудования, рассмотренными выше. Совместив эти два описания, можно увидеть, каким образом связаны модули управления и модули оборудования. Эта связь является ключевой для обеспечения общности и повторного использования компонентов в рамках управления рецептурным производством [25]. К примеру, чтобы сказать, что конкретная единица оборудования (например, клапан) является моду- 
лем управления, необходимо включить обозначающий этот клапан узел семантической сети во множество модулей управления, как показано на рисунке 16.

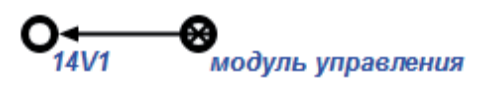

Рисунок 16 - Семантическое представление клапана - модуля управления

В результате подобных преобразований получаем фрагмент формализованной семантической сети, например представленный на рисунке 17.

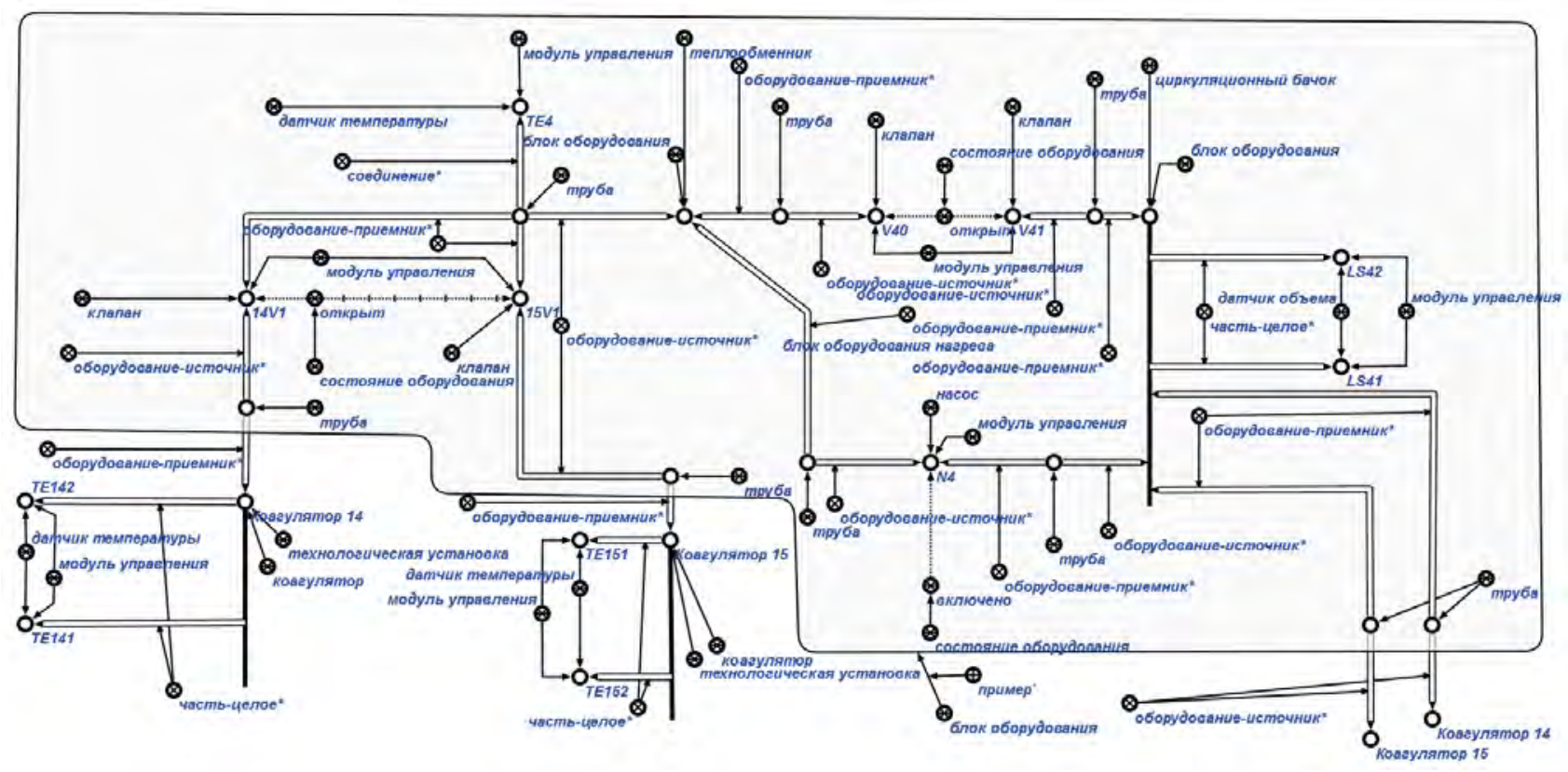

Рисунок 17 - Фрагмент формализованной семантической сети, построенной на основе исходной технологической документации

Представленная таким образом исходная документация позволяет осуществить различные запросы. Например, на запрос: «Найти блок оборудования, содержащий теплообменник» используется sc-агент поиска по произвольному образцу. Его аргументом является sс-узел, обозначающий образец поиска, приведённый на рисунке 18. Пример ответа на этот запрос показан на рисунке 19 в виде фрагмента семантической сети.

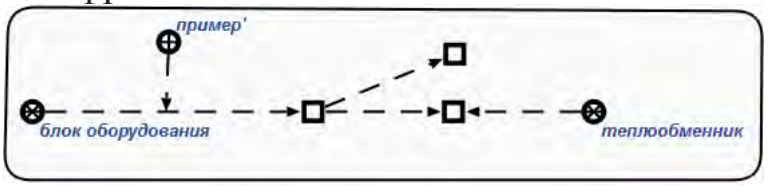

Рисунок 18 - Пример образца поиска

\section{4. Процедурная модель предприятия «Савушкин продукт»}

Процедурная модель предприятия исследуется в рамках ПрО моделей процедурного управления оборудованием рецептурных производств. В данной ПрО объектом исследования является класс процедурных элементов (procedural element) - составных частей процедурной модели. По уровню детализации различаются следующие их разновидности - процедуры производственного цеха (process cell procedure), процедуры технологических установок (unit procedure), операции (operation) и фазы (phase). Кроме того, процедурные элементы могут относиться как к оборудованию - процедурный элемент оборудования (equipment proce- 
dural element), так и к рецептуре (recipe) - рецептурный элемент оборудования (recipe procedural element).

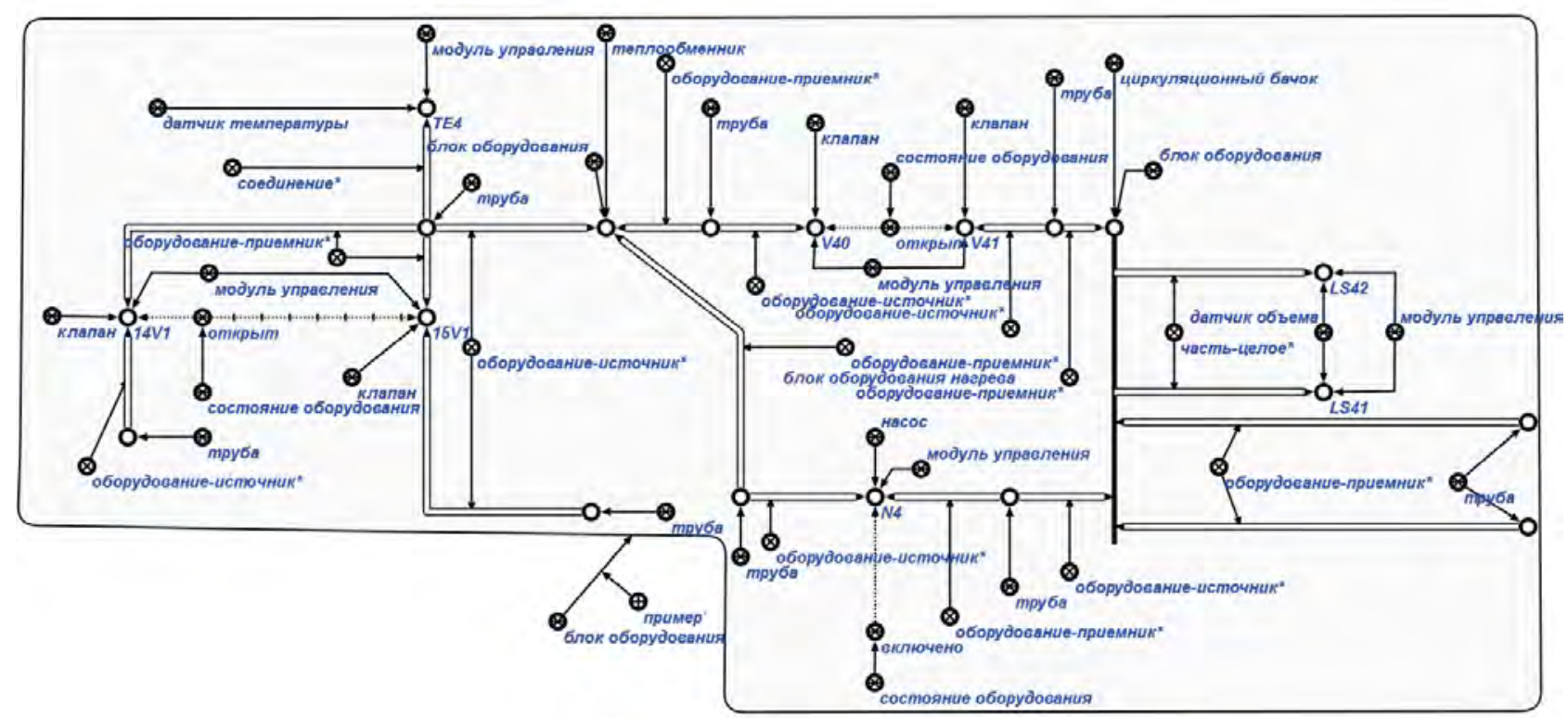

Рисунок 19 - Пример ответа системы на запрос о блоке оборудования, содержащем теплообменник

В качестве примера приведём фрагмент процедурной модели производства творога в цехе мягких сыров и творога ОАО «Савушкин продукт», выполненный средствами [26]. Процедура изготовления творога «Хуторок» заключается в последовательном выполнении процедур технологических установок приготовления, формовки и фасовки (см. левую треть рисунка 20). Unit procedure приготовления творожной массы включает, среди прочих, операции наполнения, нагрева и мойки (см. центральную часть рисунка 20). Операция нагрева включает единственную одноименную фазу, что видно в правой трети рисунка 20.

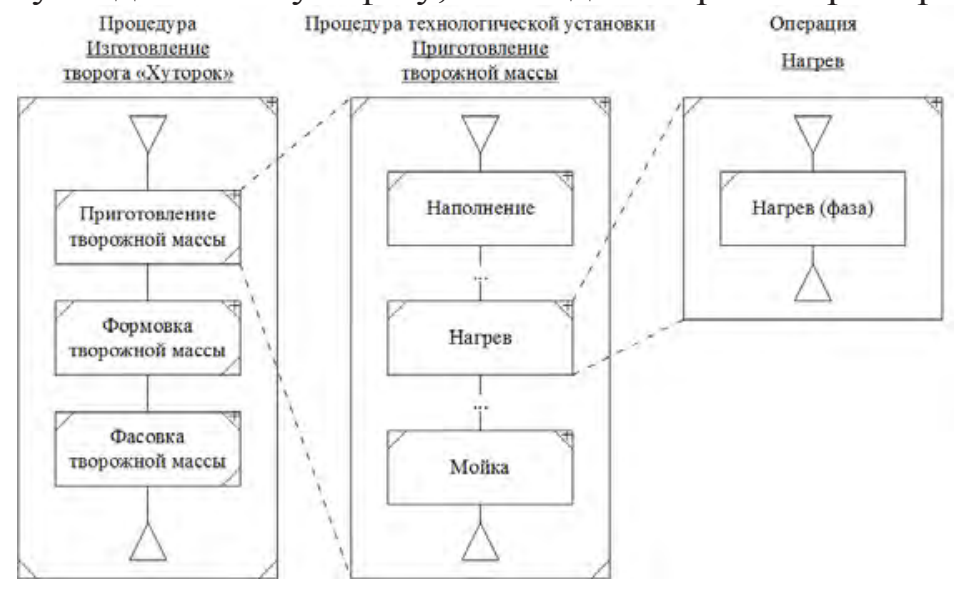

Рисунок 20 - Процедурная модель, выполненная в соответствии с ISA-88.00.02

В рамках формального представления каждому процедурному элементу будет соответствовать узел семантической сети. Конкретная разновидность процедурного элемента указывается аналогично тому, как было рассмотрено указание типа оборудования в описании модели оборудования. Процедурные элементы разного уровня детализации связываются между собой отношением декомпозиция*. Последовательность выполнения процедурных элементов в рамках процедурного элемента более высокого уровня задаётся связками отношения 
порядок исполнения *. После выполнения указанных преобразований получается фрагмент семантической сети, изображённый на языке SCg на рисунке 21.

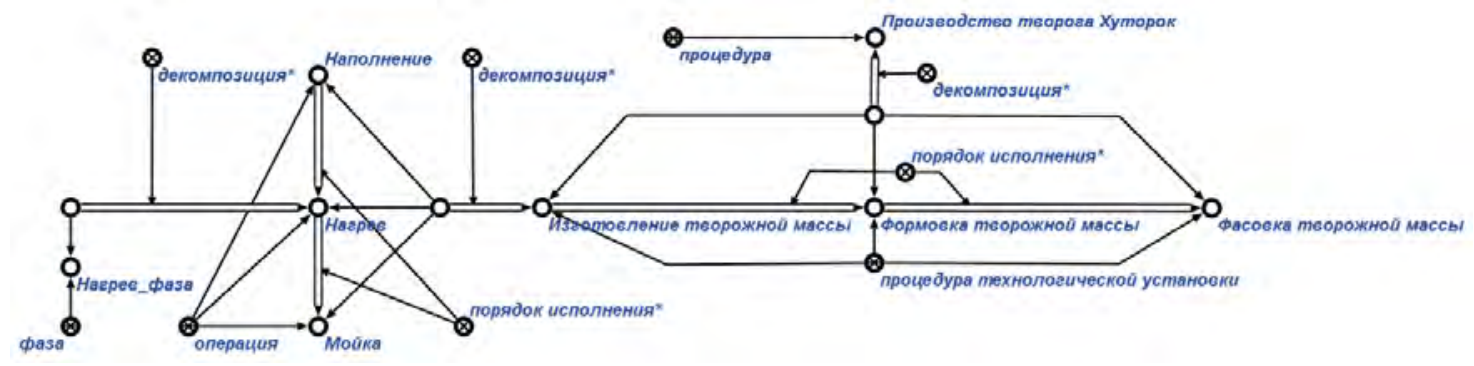

Рисунок 21 - Формализованное представление процедурной модели производства творога

\section{Заключение}

В работе показаны основные принципы онтологического проектирования предприятий рецептурного производства на примере предприятия «Савушкин продукт».

Ключевые положения работы.

- Предприятие рассматривается как интеллектуальная многоагентная система, управляемая знаниями, хранимыми в памяти, которая является общей для всех агентов этой системы и называется корпоративной памятью предприятия. Агентами такого интеллектуального предприятия являются как программные агенты, работающие над указанной корпоративной памятью, так и сотрудники предприятия, работающие и взаимодействующие через эту память.

- Основой онтологического проектирования предприятия является целостная система формальных онтологий, каждая из которых описывает принципы того или иного аспекта организации деятельности предприятий заданного класса. Совокупность моделей, которые описывают предприятие, составляют интегрированную онтологическую модель проектируемого предприятия.

- Принципы организации деятельности, направленной на совершенствование и адаптацию предприятия к изменяющимся условиям, должны быть описаны в виде онтологии совериенствования предприятий заданного класса, которая должна быть декомпозирована на несколько частных онтологий, описывающих совершенствование различных аспектов структуризации или деятельности предприятия.

- Основным критерием качества разработанной системы формальных онтологий является возможность декомпозиции онтологии совершенствования предприятий, позволяющей совершенствование различных аспектов предприятия независимо друг от друга. Этот критерий характеризует степень гибкости предприятий, проектируемых на основе указанной системы формальных онтологий.

- В основе системы формальных онтологий для предприятий рецептурного производства лежит формализация стандарта ISA-88, который представляется в виде системы следующих онтологий:

- онтологии физических моделей рецептурных производств;

- онтологии процессных моделей рецептурных производств;

- онтологии моделей процедурного управления оборудованием рецептурных производств;

- онтологии деятельности по управлению рецептурным производством.

Важно подчеркнуть высокое качество стандарта ISA-88, позволяющее проектировать предприятия рецептурного производства, обладающие высокой степенью гибкости. 


\section{Список источников}

[1] Гаврилова, T.A. Интеллектуальные технологии в менеджменте: инструменты и системы / Т.А. Гаврилова, Д.И. Муромцев // Учеб. пособие. - СПб: «Высшая школа менеджмента», 2008.

[2] Шведин, Б.Я. Онтология предприятия: экспириентологический подход: Технология построения онтологической модели предприятия / Б.Я. Шведин. - М: Ленанд, 2010. - 240 с.

[3] Боргест, Н.M. Онтология проектирования: теоретические основы. Часть 1. Понятия и принципы / Н.М. Боргест // Учеб. пособие. - Самара: Изд-во СГАУ, 2010. - 88 с.

[4] Тузовский, А.Ф. Системы управления знаниями (методы и технологии) / А.Ф. Тузовский, С.В. Чириков, В.3. Ямпольский // Под общ. ред. В.З. Ямпольского. $\square$ Томск: Изд-во НТЛ, 2005. - 260 с.

[5] Поспелов, Д.А. Принципы ситуационного управления / Д.А. Поспелов // Известия АН СССР, Техническая кибернетика. - 1971. - №2. - С. 10-17.

[6] Клыков, Ю.И. Ситуационное управление большими системами / Ю.И. Клыков. - М.:Энергия. - 1974. $134 \mathrm{c.}$

[7] Поспелов, Д.А. Ситуационное управление. Теория и практика / Д.А. Поспелов. - М.: Наука, $1986 .-284$ с.

[8] Скобелев, П.О. Онтологии деятельности для ситуационного управления предприятиями в реальном времени / П.О. Скобелев // Онтология проектирования. - 2014. - №1. - c.6-38.

[9] Терехин, Д.Э. Системы ситуационного управления на основе технологий Semantic Web / Д.Э. Терехин, А.Ф. Тузовский // «Знания - Онтологии - Теории» (ЗОНТ-15): Мат-лы V Всероссийской конференции. Новосибирск, Институт математики СО РАН, 2015. - с.151-155.

[10] Russel, S.J. Artificial Intelligence: A Modern Approach. 3rd Edition / S.J. Russel, P. Norvig. - Prentice Hall, 2010. - 1152 p.

[11] Тарасов, В.Б. От многоагентных систем к интеллектуальным организациям / В.Б. Тарасов // Эдиториал УPCC, 2002. - $352 \mathrm{c}$.

[12] Hammer, M. Reengineering Work: Don't Automate, Obliterate / M. Hammer. - Harvard Business Review, JulyAugust, 1990. - p. 104-112.

[13] Алиев, Р.А. Производственные системы с искусственным интеллектом / Р.А. Алиев, Н.М. Абдикеев, М.М. Шахназаров. - М.: Радио и связь, 1990. - 264 с.

[14] Голенков, В.B. Проект открытой семантической технологии компонентного проектирования интеллектуальных систем. Часть 1: Принципы создания / В. В. Голенков, Н.А. Гулякина // Онтология проектирования. - 2014. - №1. - с.42-64.

[15] Голенков, В.B. Проект открытой семантической технологии компонентного проектирования интеллектуальных систем. Часть 2: Унифицированные модели проектирования / В. В. Голенков, Н.А. Гулякина // Онтология проектирования. - 2014. - №4. - с.34-53.

[16] Давыденко, И.Т. Средства структуризации семантических моделей баз знаний / И.Т. Давыденко, Н.В. Гракова, Е.С. Сергиенко, А.В. Федотова // Открытые семантические технологии проектирования интеллектуальных систем (OSTIS-2016): материалы VI Междунар.научн.-техн.конф. - Мн.: БГУИР, 2016. - с. 93-106.

[17] Шункевич, Д.В. Смысловая модель обработки знаний в интеллектуальных системах / Д.В. Шункевич, К.В. Русецкий // Электроника-инфо. - 2014. - № 3. - с. 35-37.

[18] ISA88, Batch Control [Электронный pecypc]. - Режим доступа: https://www.isa.org/isa88. - Дата доступа: 20.11.2016.

[19] Parshall, J. Applying S88: Batch Control from a user's perspective / J. Parshall and L. Lamb. - ISA, 1999. $171 \mathrm{p}$.

[20] ANSI/ISA-88.00.01-2010 Batch Control Part 1: Models and Terminology. - ISA, 2010. - 158 p.

[21] ГОСТ Р МЭК 61512-1-2016. Управление серийным производством. Часть 1. Модели и терминология. - М.: Стандартинформ, 2016. - 70 с.

[22] Метасистема IMS [Электронный ресурс]. - Режим доступа: http://ims.ostis.net. - Дата доступа: 24.11.2016

[23] ГОСТ 21.404-85. Система проектной документации для строительства. Автоматизация технологических процессов. Обозначения условные приборов и средств автоматизации в схемах. - М.: Стандартинформ, 2007. - 12 c.

[24] Gamma, E. Design patterns: elements of reusable object-oriented software / E. Gamma, R. Helm, R. Johnson, J. Vlissides. - Addison-Wesley, 1995. - 395 p.

[25] JBF: Introduction to S88. For the improvement of the design of batch systems. - 2007. - 56 p.

[26] ISA-88.00.02-2001 Batch Control Part 2: Data Structures and Guidelines for Languages. - ISA, $2001 .-124$ p. 


\title{
DESIGNING BATCH-MANUFACTURING ENTERPRISES USING ONTOLOGIES
}

\author{
V.V.Golenkov ${ }^{1}$, V.V.Taberko ${ }^{2}$, D.S. Ivanyuk ${ }^{2}$, K.V. Rusetski ${ }^{1}$, D.V. Shunkevich ${ }^{1}$, \\ I.T. Davydenko ${ }^{1}$, V.V. Zakharov ${ }^{1}$, V.P. Ivashenko ${ }^{1}$, D.N. Koronchik ${ }^{1}$ \\ ${ }^{1}$ Belarusian State University of Informatics and Radioelectronics, Minsk, Belarus \\ golen@bsuir.by \\ ${ }^{2}$ JSC «Savushkin product», Brest, Belarus \\ id@pda.savushkin.by
}

\begin{abstract}
This article presents an ontological approach to designing batch manufacturing enterprises based on formal ontologies. Belarusian enterprise JSC «Savushkin product» is used as an example. In particular, this article covers formalization of standards that form the basis for enterprise operation, with ISA-88 used as an example. We propose to treat the ISA-88 standard as an ontology of a batch manufacturing subject domain, represented in the form of the natural language text. Formalization of a standard then boils down to mapping structure and contents of the standard to a hierarchy of subject domains and respective ontologies. Formal representation of a standard forms the basis for corporate intelligent system and simplifies automation of a wide range of tasks, such as providing informational service for employees, making enterprise adherent to standards and formal standard adherence evaluation. Fundamental new result presented in this article is comprised of using formal representation of the industry standard, implemented using graphodynamical model of knowledge representation and processing as a basis for creating an enterprise-wide intelligent system.
\end{abstract}

Key words: ontology, ontology-based design, intelligent system, batch manufacturing enterprise, ISA-88 standard.

Citation: Golenkov VV, Taberko VV, Ivanyuk DS, Rusetski KV, Shunkevich DV, Davydenko IT, Zakharov VV, Ivashenko VP, Koronchik DN. Designing Batch Manufacturing Enterprises Using Ontologies. Ontology of designing. 2017. 24(2): 123-144. - DOI: 10.18287/2223-9537-2017-7-2-123-144.

\section{References}

[1] Gavrilova TA, Muromtsev DI. Intelligent technologies in management: tools and systems [In Russian]. - SaintPetersburg: «Graduate School of Management»; 2008.

[2] Shvedin BYa. Enterprise ontology: an experientological approach: Technology for constructing an ontological model of an enterprise [In Russian]. - Moscow: Lenand publ.; 2010.

[3] Borgest NM. Ontology of designing: theoretical basics. Part 1. Terms and principles [In Russian]. - Samara: Samara State Aviation University publ.; 2010.

[4] Tuzovskiy AF, Tchirikov SV, Yampolskiy VZ. Knowledge management systems (methods and technologies) [In Russian]. Ed. by Yampolskiy VZ. - Tomsk: NTL publ.; 2005.

[5] Pospelov DA. Situational control principles [In Russian]. USSR AS News 1971; 2: 10-17.

[6] Klykov YuI. Situational control of large systems [In Russian]. - Moscow: Energiya publ.; 1974.

[7] Pospelov DA. Situational control. Theory and practice [In Russian]. - Moscow: Nauka publ.; 1986.

[8] Skobelev PO. Activity ontologies for realtime situational control of an enterprise [In Russian]. Ontology of Designing 2012;1(3): 6-38.

[9] Terekhin DE, Tuzovskiy AF. Situational control systems based on Semantic Web technologies [In Russian]. «Knowledge - Ontologies - Theories»: Proc. of $5^{\text {th }}$ Nationwide Russian Conference. - Novosibirsk: Math Institute of CO RAS; 2015: 151-155.

[10] Russel SJ, Norvig P. Artificial Intelligence: A Modern Approach (3 ed.). Prentice Hall; 2010.

[11] Tarasov VB. From multiagent systems to intelligent organizations [In Russian]. Editorial URSS; 2002.

[12] Hammer M. Reengineering Work: Don't Automate, Obliterate. Harvard Business Review; 1990: 68.4: $104-112$.

[13] Aliyev RA, Abdikeyev NM, Shakhnazarov MM. Enterprise systems with artificial intelligence [In Russian]. Moscow: Radio i svyaz publ.; 1990.

[14] Golenkov VV, Guliakina NA. Project of Open Semantic Technology of the Componental Design of Intelligent Systems. Part 1: The Principles of Creation [In Russian]. Ontology of designing 2014; 1(11): 42-64.

[15] Golenkov VV, Guliakina NA. Project of Open Semantic Technology of the Componental Design of Intelligent Systems. Part 2: Unified Model Design [In Russian]. Ontology of designing 2014; 4(14): 34-53. 
[16] Davydenko IT, Grakova NV, Sergienko ES, Fedotova AV. The Tools of Structuring the Semantic Models of Knowledge Bases [In Russian]. Open Semantic Technologies for Intelligent Systems (OSTIS-2016): proceedings of the $6^{\text {th }}$ international scientific and technical conference. - Minsk: BSUIR; 2016: 93-106.

[17] Shunkevich DV, Rusetski KV. Sense model of knowledge processing in intelligent systems. Electronics-info 2014; 3(105): 35-38.

[18] ISA88, Batch Control. - 2017. - URL: https://www.isa.org/isa88. - Valid on: 01.03.2017.

[19] Parshall J, Lamb L. Applying S88: Batch Control from a user's perspective. ISA; 1999.

[20] ANSI/ISA-88.00.01-2010 Batch Control Part 1: Models and Terminology. ISA; 2010.

[21] GOST 61512-1-2016. Batch Control Part 1: Models and Terminology [In Russian]. - Moscow: Standartinform; 2016.

[22] IMS Metasystem. - 2017. - URL: http://ims.ostis.net. - Valid on: 01.03.2017.

[23] GOST 21.404-85. System of design documents for construction. Industrial process automation. Instrumentation symbols for use in diagrams [In Russian]. - Moscow: Standartinform; 2007.

[24] Gamma E, Helm R, Johnson R, Vlissides J. Design patterns: elements of reusable object-oriented software. Addison-Wesley; 1995.

[25] JBF: Introduction to S88. For the improvement of the design of batch systems. JBF; 2007.

[26] ISA-88.00.02-2001 Batch Control Part 2: Data Structures and Guidelines for Languages. ISA; 2001.

\section{Сведения об авторах}

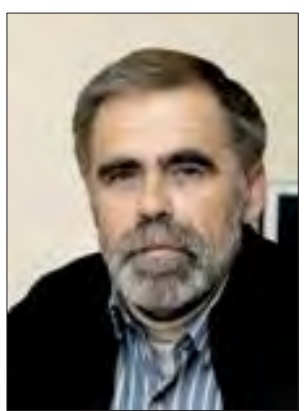

Голенков Владимир Васильевич, 1949 г. рождения. В 1971 г. с отличием окончил физический факультет Белорусского государственного университета, д.т.н. (1996), профессор, заведующий кафедрой интеллектуальных информационных технологий Белорусского государственного университета информатики и радиоэлектроники, инициатор ежегодной международной научно-технической конференции «Открытые семантические технологии проектирования интеллектуальных систем», член Российской ассоциации искусственного интеллекта, член редколлегий журналов «Программные продукты и системы», «Онтология проектирования», «Электроника ИНФО», «Речевые технологии». В списке научных трудов более 100 работ в области семантических технологий.

Vladimir Golenkov (b. 1949). In 1971 he graduated from the physics department of Belarusian State University with honors diploma, Dr. of science (1996), professor. The head of the department of Intelligent information technologies of Belarusian State University of Informatics and Radioelectronics, initiator of the annual international science and technical conference «Open Semantic technologies for Intelligent Systems» (OSTIS), member of Russian association of artificial intelligence, editorial board member of such journals as «Program products and systems», «Ontology of designing», «Electronics-INFO», «Speech technologies». The list of his published works consists of more than 100 articles on the various aspects of semantic approach in the intelligent systems design.

Таберко Валерий Васильевич, 1963 г. рождения. В 1985 г. окончил факультет прикладной математики Белорусского государственного университета. С 1985 г. до 1993 г. работал на Брестском электромеханическом заводе, в 1993 г. перешел на предприятие «Брестский молочный комбинат» начальником информационно-вычислительного центра, в настоящее время занимает должность заместителя генерального директора по логистике на ОАО «Савушкин продукт».

Valery Taberko (b. 1963). In 1985 he graduated from the faculty of Applied Mathematics of Belarusian State University. Since 1985 till 1993 worked at Brest electromechanical plant, in 1993 moved to a new job at Brest milk plant as Head of Information and Computing Center, now works as Deputy Director for Logistics at JSC «Savushkin product».
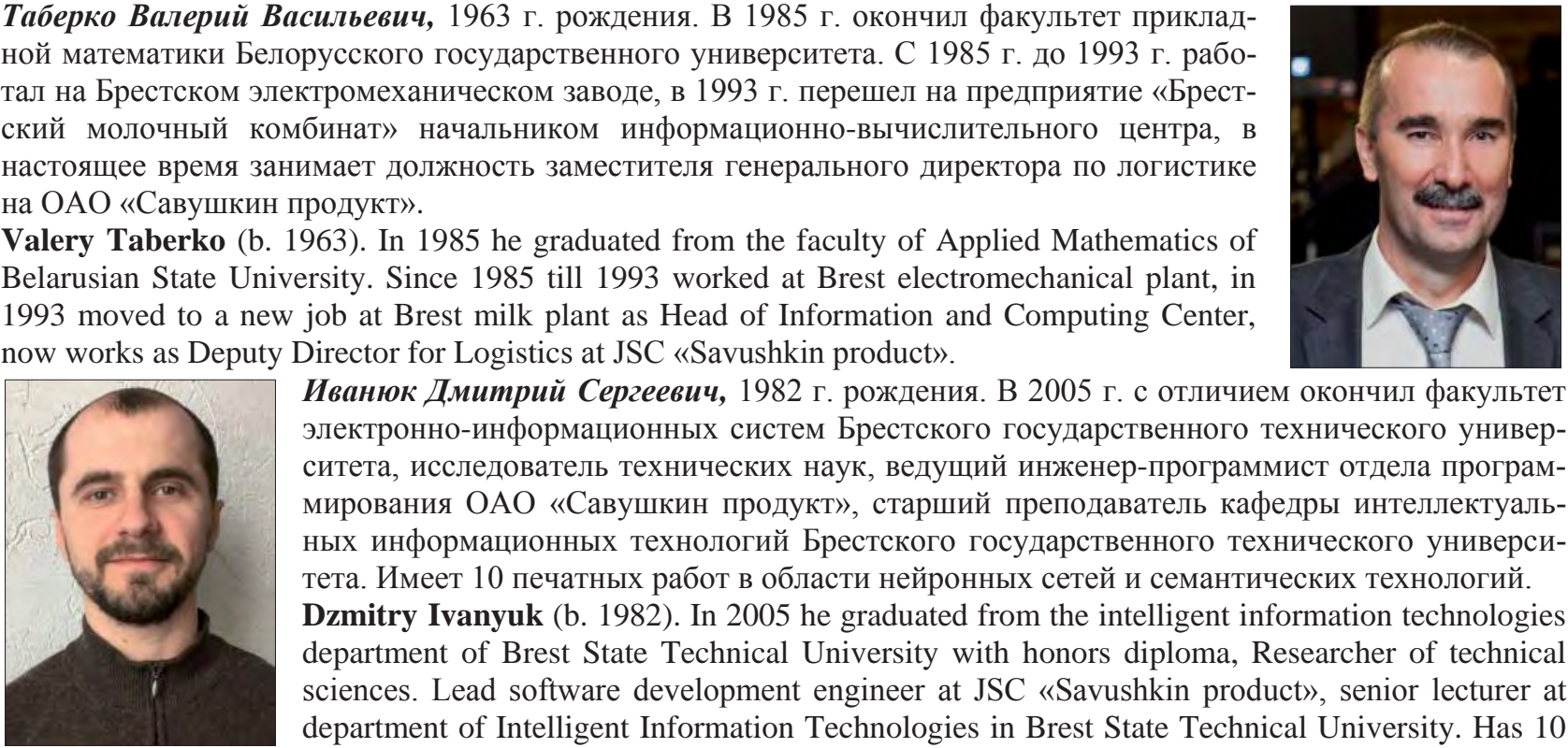

Иванюк Дмитрий Сергеевич, 1982 г. рождения. В 2005 г. с отличием окончил факультет электронно-информационных систем Брестского государственного технического университета, исследователь технических наук, ведущий инженер-программист отдела программирования ОАО «Савушкин продукт», старший преподаватель кафедры интеллектуальных информационных технологий Брестского государственного технического университета. Имеет 10 печатных работ в области нейронных сетей и семантических технологий. Dzmitry Ivanyuk (b. 1982). In 2005 he graduated from the intelligent information technologies department of Brest State Technical University with honors diploma, Researcher of technical sciences. Lead software development engineer at JSC «Savushkin product», senior lecturer at department of Intelligent Information Technologies in Brest State Technical University. Has 10 
published works on the subject of semantic technologies and artificial neural networks.

Русецкий Кирилл Валерьевич, 1990 г. рождения. В 2013 г. окончил Белорусский государственный университет информатики и радиоэлектроники по специальности «Искусственный интеллект», магистр технических наук, аспирант кафедры интеллектуальных информационных технологий Белорусского государственного университета информатики и радиоэлектроники. Имеет около 20 печатных работ в области семантических технологий.

Kirill Rusetski (b. 1990). In 2013 he graduated from Belarusian State University of Informatics and Radioelectronics majoring in Artificial intelligence. Master of technical sciences. Post graduate student of Belarusian State University of Informatics and Radioelectronics. Has near 20 published works on the subject of semantic technologies.
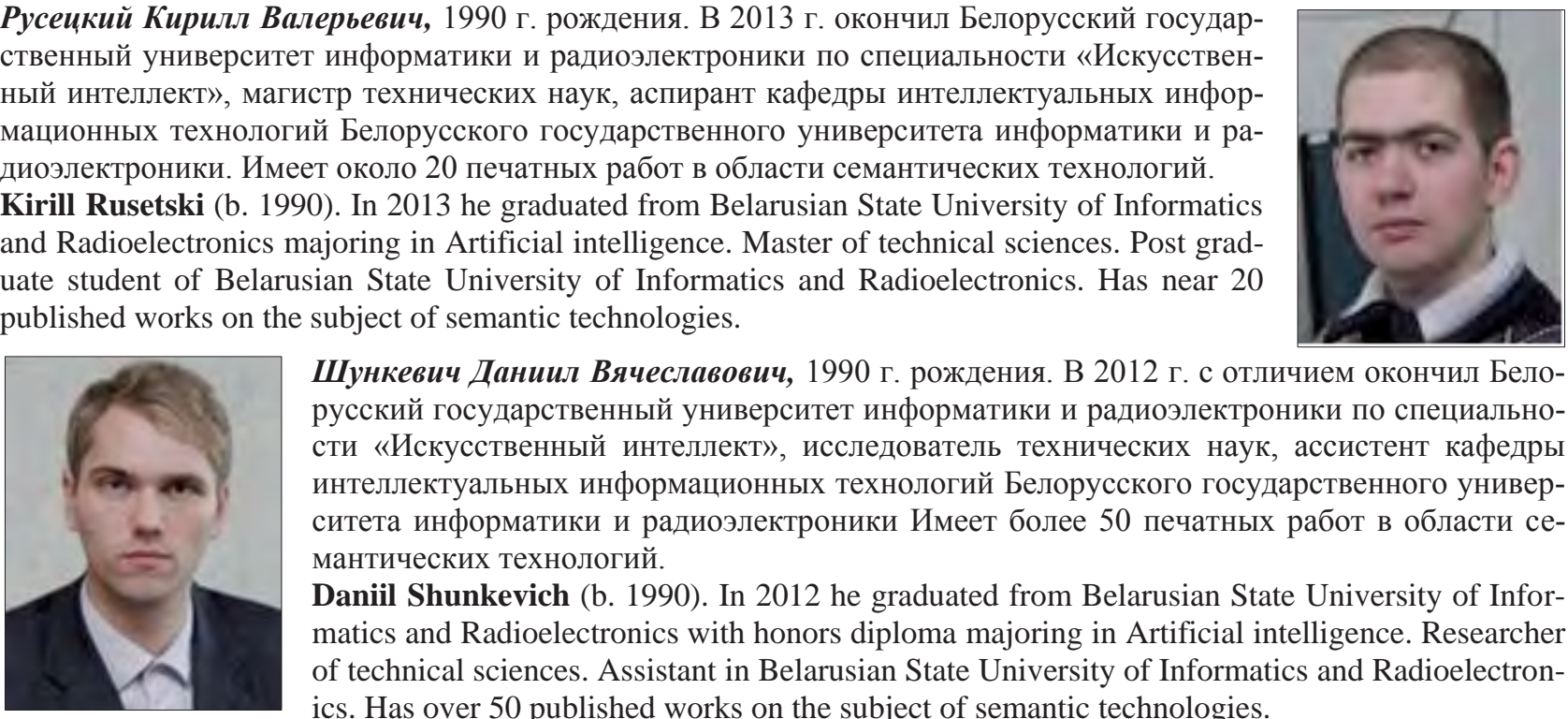

Шункевич Даниил Вячеславович, 1990 г. рождения. В 2012 г. с отличием окончил Белорусский государственный университет информатики и радиоэлектроники по специальности «Искусственный интеллект», исследователь технических наук, ассистент кафедры интеллектуальных информационных технологий Белорусского государственного университета информатики и радиоэлектроники Имеет более 50 печатных работ в области семантических технологий.

Daniil Shunkevich (b. 1990). In 2012 he graduated from Belarusian State University of Informatics and Radioelectronics with honors diploma majoring in Artificial intelligence. Researcher of technical sciences. Assistant in Belarusian State University of Informatics and Radioelectronics. Has over 50 published works on the subject of semantic technologies.

Давыденко Ирина Тимофеевна, 1987 г. рождения. В 2010 г. окончила Белорусский государственный университет информатики и радиоэлектроники по специальности «Искусственный интеллект», исследователь технических наук, ассистент кафедры интеллектуальных информационных технологий Белорусского государственного университета информатики и радиоэлектроники Имеет более 60 печатных работ в области семантических технологий.

Irina Davydenko (b. 1987). In 2010 she graduated from Belarusian State University of Informatics and Radioelectronics majoring in Artificial intelligence. Researcher of technical sciences. Assistant in Belarusian State University of Informatics and Radioelectronics. Has over 60 published works on the subject of semantic technologies.

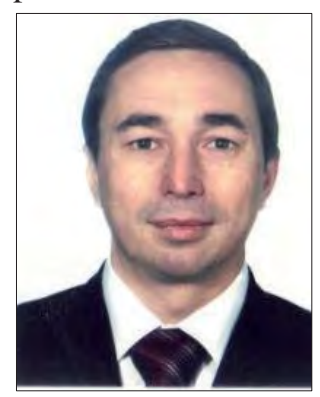

Захаров Владимир Владимирович, 1964 г. рождения. В 1986 г. Окончил Минское высшее инженерное зенитное ракетное училище ПВО, кандидат технических наук (2004), доцент кафедры интеллектуальных информационных технологий Белорусского государственного университета информатики и радиоэлектроники. Имеет более 40 печатных работ в области синтеза спецпроцессоров и защиты информации.

Vladimir Zakharov (b. 1964). In 1986 he graduated from Minsk Higher Air Defense Antiaircraft Missile Academy. PhD (2004). Assistant professor of Belarusian State University of Informatics and Radioelectronics. Has over 40 published works on the subject of synthesis of special processors and information protection.

Ивашенко Валерьян Петрович, 1978 г. рождения. В 2000 г. окончил Белорусский государственный университет информатики и радиоэлектроники по специальности «Искусственный интеллект», кандидат технических наук (2015), доцент кафедры интеллектуальных информационных технологий Белорусского государственного университета информатики и радиоэлектроники Имеет более 50 печатных работ в области семантических технологий.

Valerian Ivashenko (b. 1978). In 2000 he graduated from Belarusian State University of Informatics and Radioelectronics majoring in Artificial intelligence. PhD (2015). Assistant pro-

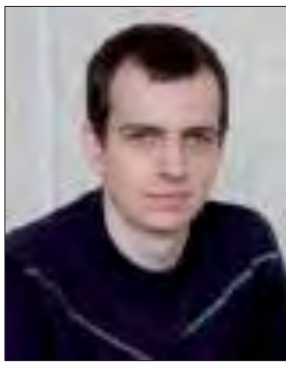
fessor in Belarusian State University of Informatics and Radioelectronics. Has over 50 published works on the subject of semantic technolo-

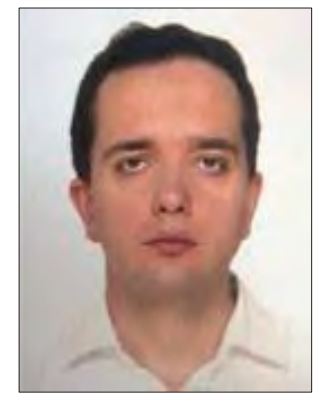
gies.

Корончик Денис Николаевич, 1987 г. рождения. В 2009 г. окончил Белорусский государственный университет информатики и радиоэлектроники по специальности «Искусственный интеллект», исследователь технических наук. Имеет около 10 печатных работ в области семантических технологий.

Denis Koronchik (b. 1987). In 2009 he graduated from Belarusian State University of Informatics and Radioelectronics majoring in Artificial intelligence. Researcher of technical sciences. Has over 10 published works on the subject of semantic technologies. 


\title{
УДК 004.89, 004.6, 614
}

\section{СЛИЯНИЕ МЕДИЦИНСКИХ ДАННЫХ НА ОСНОВЕ ОНТОЛОГИЙ}

\author{
С.В. Лебедев ${ }^{1}$, Н.А. Жукова ${ }^{1,2}$ \\ ${ }^{1}$ Санкт-Петербургский государственный электротехнический университет «ЛЭТИ» \\ им. В.И. Ульянова (Ленина), Санкт-Петербург, Россия \\ lebedev.sv.etu@gmail.com \\ ${ }^{2}$ Санкт-Петербургский национальный исследовательский университет информаичионных технологий, \\ механики и оптики, Санкт-Петербург, Россия \\ nazhukova@mail.ru
}

\begin{abstract}
Аннотация
В информационных системах медицинских учреждений хранится большой объём данных, относящихся к проводимым лечебно-диагностическим мероприятиям. Собираемые данные представляют собой слабосвязанный и слабоструктурированный массив. Для повышения эффективности информационной поддержки лечения необходимо внедрить в информационные медицинские системы процесс непрерывного слияния данных и знаний. В статье для реализации этого процесса предложен подход, в котором совместно используются модель слияния данных JDL (Joint Directors of Laboratories) и онтологии. Модель определяет состав этапов процесса слияния данных, их функции и взаимосвязь. Онтологии используются для представления и связывания отдельных наборов данных и знаний. Для детализации этапов и проверки подхода в целом рассмотрен упрощённый сценарий слияния данных и знаний. Сценарий разработан по запросу специалистов Северо-Западного федерального медицинского исследовательского центра имени В.А. Алмазова Минздрава России, который является одним из ведущих центров в области кардиологии (Россия, Санкт-Петербург). Сценарий включает несколько этапов, состав которых определён уровнями JDL модели. Предварительно строятся собственные медицинские онтологии. На первом этапе из исходных неструктурированных данных выделяются отдельные объекты - лекарства и диагнозы для каждого пациента. На втором этапе извлечённые объекты связываются с элементами собственных и сторонних онтологий. На третьем этапе на основе установленных связей выявляются пары взаимодействующих лекарств. На четвёртом этапе формируется графическое представление данных. Сценарий позволил подтвердить применимость подхода для слияния медицинских данных, уточнить детали реализации каждого из этапов, определить набор технологий для реализации. В статье предложен новый подход к совместному использованию модели слияния данных и онтологий, позволяющий повысить связанность данных в медицинских информационных системах.
\end{abstract}

Ключевые слова: онтология, модель слияния данных, медицинские информациионые системы, открытые связанные данные.

Цитирование: Лебедев, С.В. Слияние медицинских данных на основе онтологий / С.В. Лебедев, Н.А. Жукова // Онтология проектирования. - 2017. - Т. 7, №2(24). - С. 145-159. - DOI: 10.18287/2223-9537-2017-7-2-145-159.

\section{Введение}

В повседневной работе медицинских учреждений накапливается большой объём лечебно-диагностических данных. К ним относятся экспертные заключения профильных врачей, результаты проведения диагностических исследований, дневники лечащих врачей и т.п. Медицинские информационные системы (МИС), которые используется для управления этими данными, обладают рядом недостатков [1]: 
- накапливаемые данные слабо структурированы и слабо связаны как между собой, так и с имеющимися базами знаний;

- МИС различных учреждений не связаны между собой.

Для эффективной информационной поддержки лечебно-диагностических мероприятий необходимо внедрить в МИС процесс непрерывного связывания медицинских данных и знаний. На вход процесса могут поступать данные в разных форматах и из различных источников. Результатом должно быть единое представление, которое можно было бы использовать, например, для прогноза течения болезни, планирования лечения и т.п.

Формат представления данных, используемый в таких процессах, должен отвечать следующим требованиям.

- Быть доступным для машинной обработки. Это позволит применять алгоритмы обработки и анализа данных, строить информативные графические представления (графы, таблицы, диаграммы и т.п.), использовать сложные алгоритмы поиска зависимостей и извлечения знаний.

- Быть достаточно выразительным для описания предметной области (ПрО) медицины.

- Позволять связывать разнородные массивы данных и базы знаний.

Таким образом, для решения проблемы необходимо решить две задачи:

- выбрать и адаптировать модель слияния данных;

- выбрать средства для представления и связывания данных и знаний.

\section{1 Текущее состояние области слияния медицинских данных}

Рассмотрим существующие решения поставленных задач. В [2] описаны модели процессов слияния данных:

- цикл OODA (O - observe, O - orient, D - decide, A - act);

- модель слияния данных JDL (Joint Directors of Laboratories);

- модель ситуационной осведомленности Эндсли и др.

Для реализации предлагаемого подхода выбрана модель JDL как наиболее общая. Подробное описание модели можно найти в [3, 4]. Модель JDL включает следующие уровни: уровень работы с сырыми данными, уровень извлечения объектных данных, уровень построения ситуации, уровень вычисления последствий и уровень взаимодействия с пользователем. Для каждого уровня описаны функции и способ взаимодействия с соседними уровнями. Модель не ограничивает способы реализации уровней и не привязана к какой-либо ПрО.

Один из вариантов применения JDL модели в области медицины представлен в [5]. Авторы статьи используют модель для объединения методов анализа медицинских данных в единый вычислительный процесс. В отличие от указанной работы в предлагаемом подходе акцент сделан на применение онтологий как основы процесса слияния.

Онтологии - это центральное понятие глобальной семантической сети (semantic web), которое является одним из перспективных направлений развития МИС [6]. Онтологии позволяют формально описать ПрО, связать множество таких описаний друг с другом, использовать описание для аннотирования данных. Таким образом, данные становятся связанными как с общими знаниями, так и с другими данными, обеспечивается интерпретация данных на машинном уровне.

Ключевыми в семантической сети являются модель и схема описания метаданных (RDF/RDFS), язык описания онтологий (OWL), язык запросов к RDF-описаниям (SPARQL). Инструменты семантической сети включают редакторы, хранилища, средства отображения, средства построения систем правил, машины логического вывода и т.п. 
Некоторые общие вопросы использования технологий семантической сети в медицине рассмотрены в $[7,8]$. В частности, в работе [8] рассматривается само понятие онтологии и применение онтологий в медицине. Авторы обосновывают целесообразность совместного использования онтологий и методов интеллектуального анализа данных (data mining). Приводятся примеры инструментов, позволяющих строить онтологии по текстам на естественном языке.

В целом, задача извлечения знаний в области медицины должна решаться применительно к различным типам данных - числовым, текстовым, графическим. Основное внимание уделяется обработке текстов, т.к. значимые результаты врачебной деятельности представлены именно в таком формате. Один из подходов работы с текстами описан в [9].

Важным условием для построения процесса слияния является наличие открытых медицинских баз знаний, представленных в форматах семантической сети. Одним из проектов по созданию доступных онтологий биомедицины является OBO Foundry ${ }^{1}$ (Open Biomedical Ontologies). В этот проект входит ряд онтологий, включая онтологию человеческих болезней (Human Disease Ontology), Онтологию взаимодействия лекарств (Drug Interaction and Evidence Ontology) и др. Онтологии ОВО Foundry образуют одну из основных частей портала Национального центра биомедицинских онтологий (National Center for Biomedical Ontology, NCBO's BioPortal ${ }^{2}$ ). Портал содержит 541 онтологию. Инициатива по созданию и распространению открытых данных в рамках направления семантической сети называется omкрытылми связаннылми данныли (Linked Open Data, LOD).

B [10] описан облачный сервис, который использует открытые связанные данные для повышения эффективности оказания неотложной помощи. В частности, используется онтология Международной классификации болезней (International Classification of Diseases, ICD). Сервис организован как набор модулей, реализующих извлечение, хранение, преобразование, отображение и связывание данных.

Таким образом, семантическая сеть предоставляет искомые средства для описания и связывания медицинских данных и знаний, позволяет использовать открытые медицинские онтологии. Слияние медицинских данных на основе онтологий можно рассматривать как один из вариантов решения проблемы интеграции системы документооборота медицинского учреждения с существующими базами медицинских знаний. Решение этой проблемы необходимо, например, при автоматизации интеллектуальной деятельности на базе экспертных систем в области медицины $[11,12]$.

\section{2 Слияние медицинских данных на основе онтологий}

Конкретизируем предложенный подход, основанный на реализации JDL модели. Для этого:

1) уточним каждый из функциональных уровней JDL модели слияния данных применительно к медицинской области;

2) определим место онтологий в процессе слияния данных.

На рисунке 1 представлена уточнённая модель слияния данных. На первом уровне получают слабоструктурированные и слабосвязанные данные: результаты анализов и диагностических процедур, заключения, дневники врачей и т.п. Данные могут поступать из реляционных баз данных, браться из файлов.

На втором уровне из слабоструктурированных данных извлекают отдельные термины и числовые данные. Например, названия назначенных лекарств, поставленный основной диа-

\footnotetext{
${ }^{1}$ OBO Foundry - http://www.obofoundry.org/

${ }^{2}$ BioPortal, the world's most comprehensive repository of biomedical ontologies - https://bioportal.bioontology.org/
} 
гноз, перечень сопутствующих диагнозов, числовые показатели параметров крови. К каждому формату данных должны применяться подходящие методы.

На третьем уровне - построение ситуации - связывают извлечённые термины и данные с понятиями собственных и сторонних онтологий. Например, диагноз пациента можно привязать к соответствующему понятию из Международного классификатора болезней. Под ситуацией будем понимать множество взаимосвязанных данных, ассоциированных с конкретным пациентом.

На четверном уровне полученная ситуация используется для порождения нового знания. Например, используя онтологическое описание воздействия лекарства на течение болезни, можно спрогнозировать последствия приёма лекарства конкретным пациентом. На этом этапе могут быть использованы запросы к онтологиям, логический вывод, продукционные системы.

На пятом уровне отображают результаты двух предыдущих уровней пользователю в форме таблиц, графов, диаграмм, функциональных зависимостей.

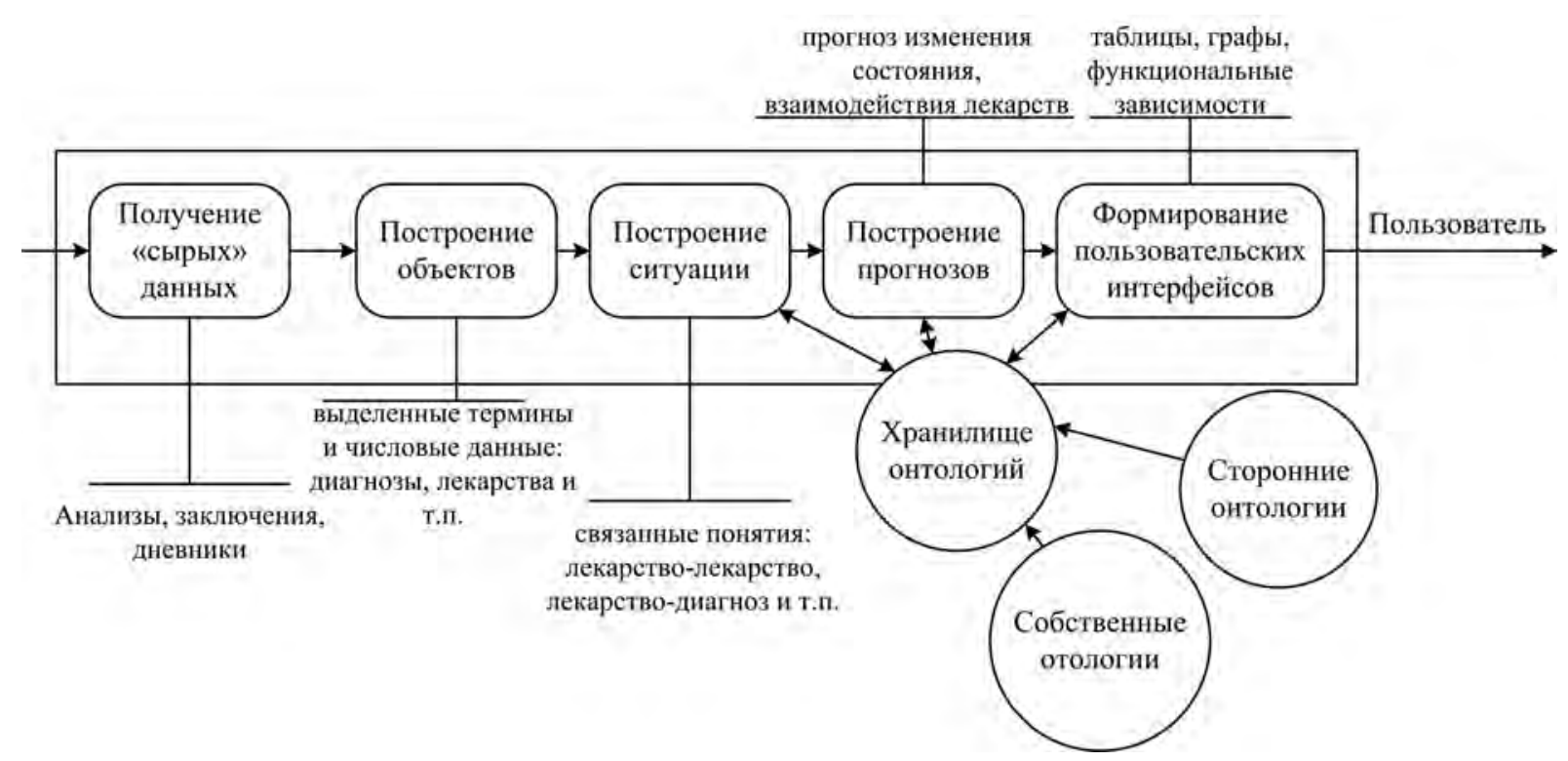

Рисунок 1 - Расширенная модель слияния медицинских данных

В представленной модели онтологии используются, начиная с третьего уровня. Путём аннотирования данные привязываются к элементам онтологии. Существующие между понятиями онтологии связи позволяют связать разрозненные данные. На четвёртом уровне установленные связи используются для получения новых знаний. На последнем уровне на основе онтологий строятся графические преставления.

\section{3 Проверка предложенного подхода}

Для проверки подхода подготовлен и реализован упрощённый сценарий поиска взаимодействия лекарств, назначенных пациенту. Сам сценарий подготовлен в интересах СевероЗападного федерального медицинского исследовательского центра имени В. А. Алмазова как демонстрация возможностей технологий семантической сети.

Цель проверки заключается в решении следующих задач:

- определить перечень инструментов и операций для реализации каждого уровня;

- выявить возможные проблемы;

- наметить пути дальнейшего развития подхода. 


\section{1 Описание сценария}

Упрощённый сквозной сценарий разделён на этапы, каждый из которых соответствует уровню JDL модели (см. рисунок 2).

На первом этапе получают файл в формате CSV (Comma Separated Values - значения, разделённые запятыми). Файл представляет собой таблицу с заголовком. Каждому пациенту соответствует несколько строк. Строки состоят из нескольких блоков неструктурированного текста. В сценарии используются блок текста истории болезни, содержащий поставленный диагноз, и блок рекомендаций, содержащий перечисление назначенных лекарств.

На втором этапе из блоков сначала извлекаются фрагменты текста, после чего из фрагментов извлекаются интересующие термины - названия лекарств и диагнозы.

На третьем этапе выполняется связывание извлечённых терминов с понятиями онтологий. Используются как собственные, так и сторонние онтологии.

На четвёртом этапе формируется и выполняется SPARQL-запрос на поиск лекарств, которые могут вступить во взаимодействие.

На пятом этапе строится граф взаимодействия лекарств, предписанных одному конкретному пациенту.

Помимо перечисленных этапов введён предварительный этап, на котором строятся собственные онтологии.

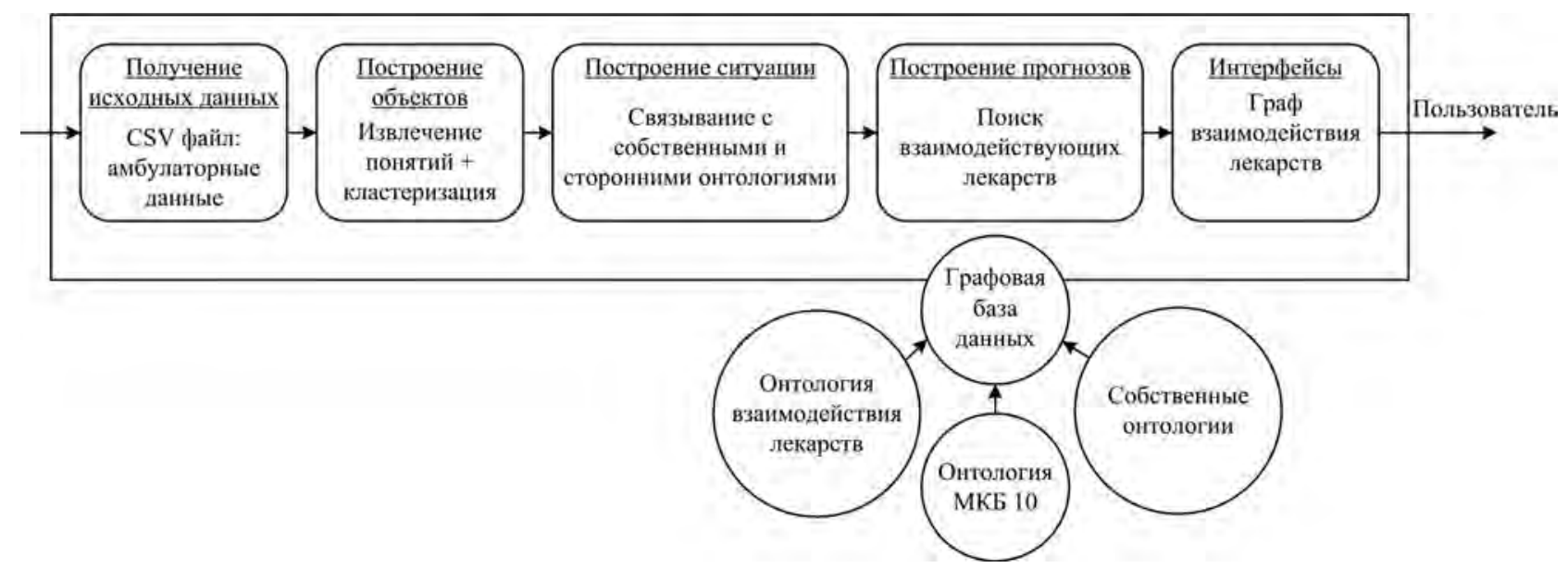

Рисунок 2 - Модель сценария слияния данных

\section{2 Используемые инструменты}

Для построения процесса слияния данных были использованы следующие инструменты:

OpenRefine ${ }^{3}$ - приложение для преобразования данных из одного формата в другой. Данные представляются в форме электронных таблиц. Для работы с RDF устанавливается специальное расширение. Расширение позволяет проводить автоматическое связывание данных с элементами выбранной онтологии и экспорт данных в формате RDF. Инструмент используется на втором и третьем этапах.

Blazegraph ${ }^{4}$ - графовая база данных. Поддерживает работу с RDF и SPARQL. Позволяет организовать SPARQL-endpoint - точку доступа для удалённого получения данных с помощью SPARQL запросов. Используется на всех этапах, начиная с третьего.

\footnotetext{
${ }^{3}$ OpenRefine - http://openrefine.org/

${ }^{4}$ Blazegraph Database - https://wiki.blazegraph.com/wiki/index.php/Main_Page
} 
M etaphactory ${ }^{5}$ - каркас для построения веб-приложений. Особенностью данного каркаса является формирование HTML-страниц на базе SPARQL-запросов. Каждая страница строится на основе фрагмента кода, включающего запрос и отображение результатов запроса на элементы графического интерфейса. Запросы выполняются к привязанной к каркасу базе Blazegraph через механизм SPARQL-endpoint. Используется на пятом этапе согласно рисунку 2.

TopBraid $^{6}$ - редактор онтологий на основе платформы Eclipse ${ }^{7}$. Поддерживает логический вывод, выполнение SPARQL-запросов, совместную разработку онтологий. Используется на предварительном этапе для построения собственных онтологий.

Схема совместного использования инструментов представлена на рисунке 3.

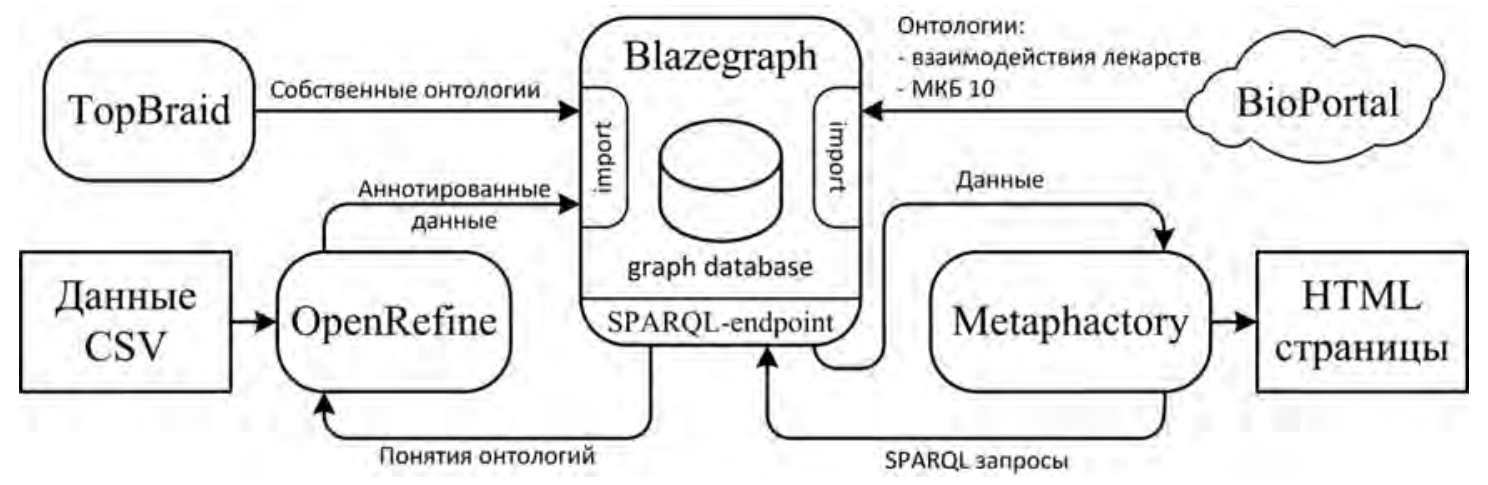

Рисунок 3 - Схема совместного использования инструментов

Перейдем к описанию отдельных этапов сценария.

\section{3 Онтологии}

В основе предлагаемого подхода лежит использование онтологий. Используются как собственные онтологии, так и сторонние. Собственные онтологии представляют единую модель, которая позволяет объединить разрозненные данные и отдельные сторонние онтологии. Собственные онтологии организованы в иерархию (см. рисунок 4) и разделены на два множества - метаонтологии и конкретные онтологии. Метаонтологии содержат общие понятия. Конкретные онтологии содержат медицинские данные, аннотированные элементами метаонтологий. Такое разделение позволяет отделить данные от общих понятий и открыто распространять последние.

Онтология Hospital объединяет все метаонтологии и представляет собой онтологию для медицинского учреждения. Онтология Diagnostics содержит понятия для аннотирования результатов различных исследований. В частности, онтология Echocardiography расширяет онтологию Diagnostics понятиями, специфическими для эхокардиографического исследования. Онтология Disease включает понятия для описания болезней. Её расширяет онтология лекарств - M edicineTreatment.

На рисунке 5 представлен фрагмент, объединяющий несколько метаонтологий. Граф визуализирован с помощью средства визуализации онтологий Ontodia ${ }^{8}$. В качестве источника данных использован SPARQL-endpoint на основе базы Blazegraph. Отметим, что названия

\footnotetext{
${ }^{5}$ Metaphactory - http://www.metaphacts.com/

${ }^{6}$ Topbraid editor - http://www.topquadrant.com/products/

${ }^{7}$ The Eclipse Foundation - https://eclipse.org/

${ }^{8}$ Ontodia toolkit - http://www.ontodia.org/
} 
конкретных болезней и лекарств были извлечены из конкретных данных и включены в состав метаонтологии, так как они относятся к общим понятиям.

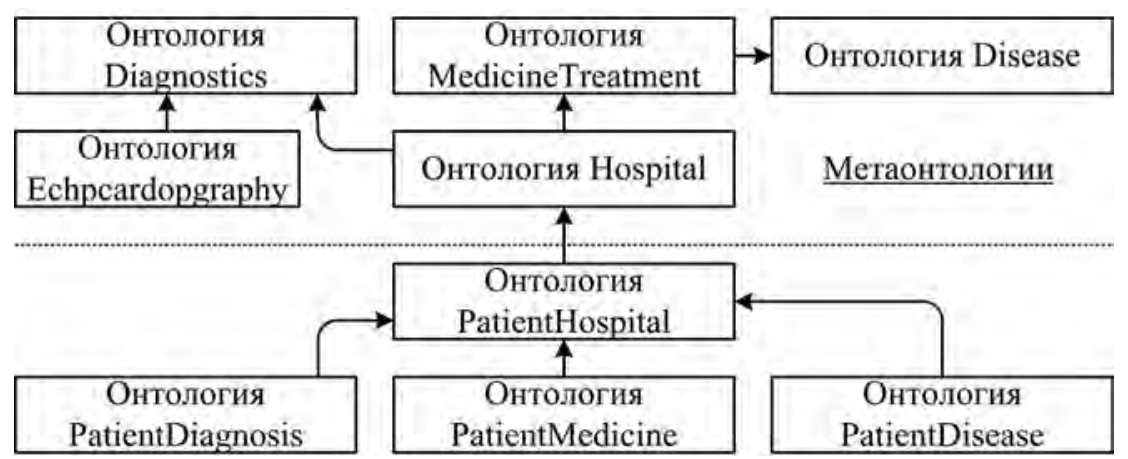

Рисунок 4 - Иерархия онтологий

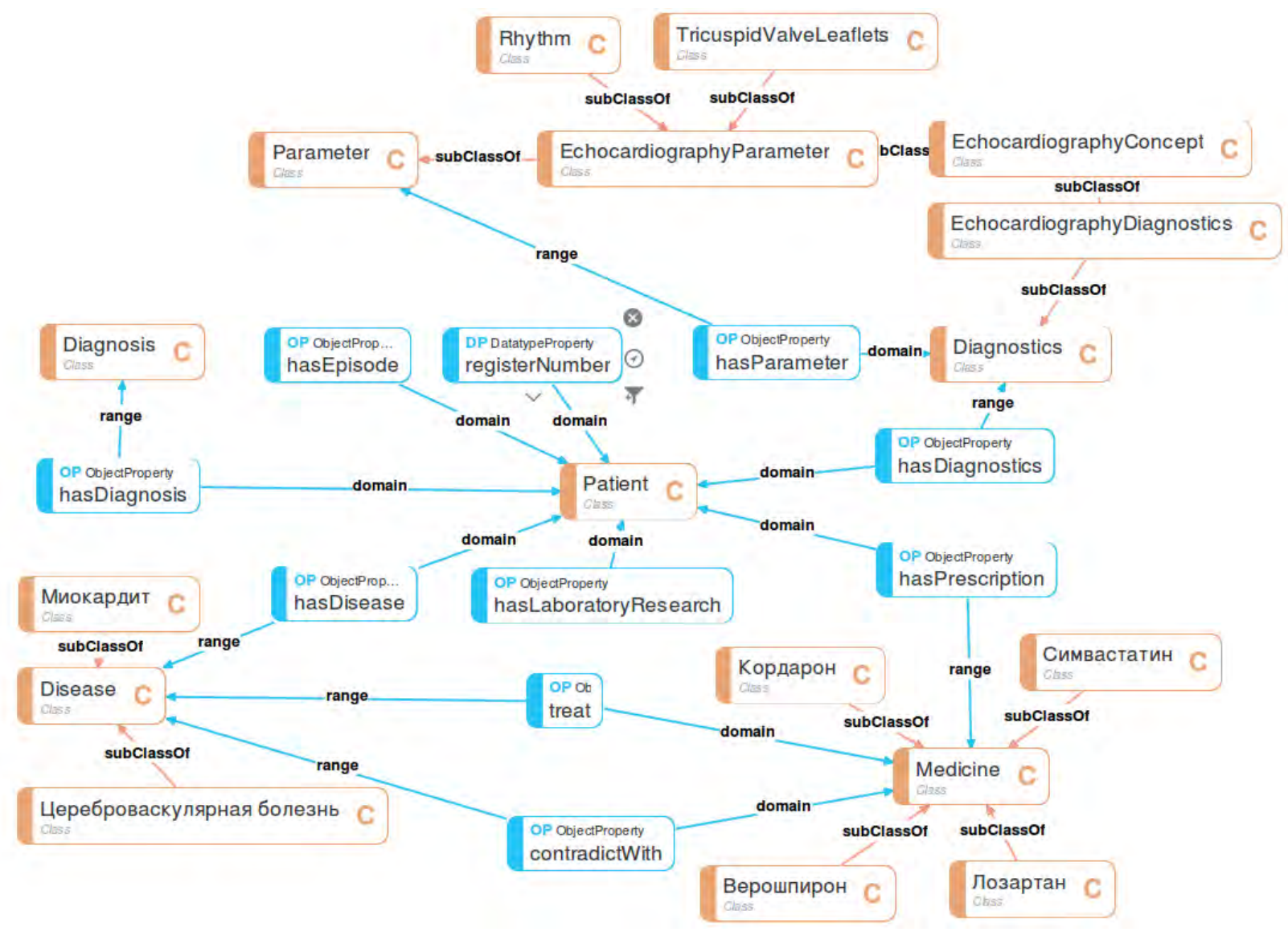

Рисунок 5 - Фрагмент метаонтологии

В качестве сторонних онтологий были использованы онтология Международной классификации болезней ${ }^{9}$ в 10 редакции и Онтология взаимодействия лекарств ${ }^{10}$.

Упомянутые онтологии используется на следующих этапах:

- $\quad$ на третьем уровне для аннотирования извлечённых терминов;

- на четвёртом уровне для поиска возможных взаимодействий лекарств;

- на пятом уровне для подготовки пользовательских представлений.

${ }_{9}^{9}$ ICD10CM ontology - http://bioportal.bioontology.org/ontologies/ICD10CM

${ }^{10}$ The Drug-Drug Interactions Ontology - https://bioportal.bioontology.org/ontologies/DINTO 


\section{4 Извлечение терминов}

На рисунке 6 представлены этапы извлечения названий отдельных лекарств из фрагмента текста. Каждый фрагмент содержит рекомендации для одного пациента в части медикаментозного лечения. В результате обработки фрагмента получаем список лекарств, которые ему назначены.

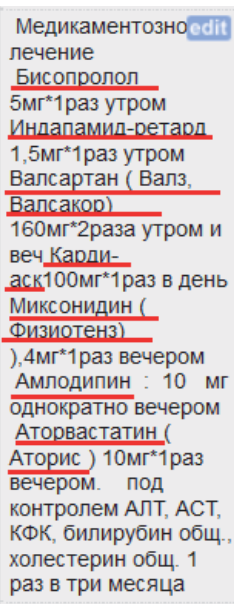

a) Исходный фрагмент

\begin{tabular}{l} 
Бисопролол edit \\
Индапамид- \\
ретард \\
Валсартан \\
Валз \\
Валсакор \\
Карди-аск \\
Моксонидин \\
Физиотенз \\
\hline Амлодипин \\
\hline Аторвастатин \\
\hline Аторис \\
\hline
\end{tabular}

б) Извлеченные названия

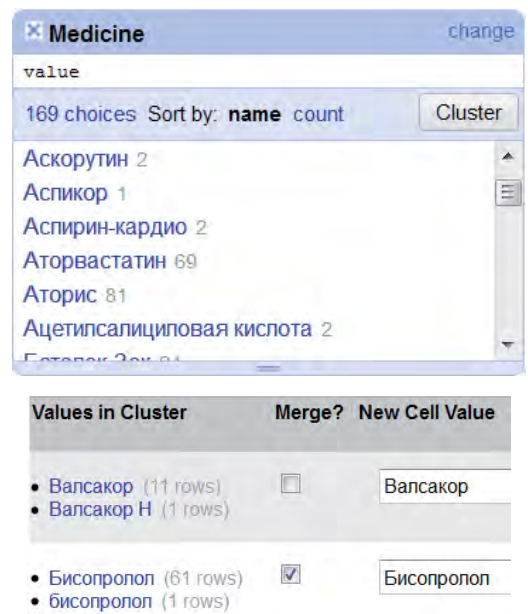

в) Словарь лекарств и унификация названий

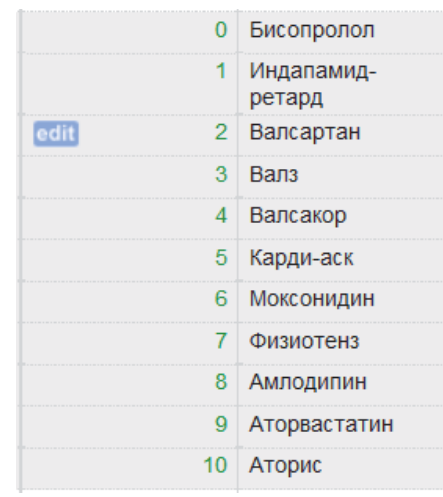

г) Нумерация лекарств

Рисунок 6 - Извлечение названий лекарств

На рисунке 6а представлен фрагмент текста, в котором выделяемые названия подчёркнуты красным. Названия извлекаются из текста с помощью операций на основе регулярных выражений, разделения и объединения данных (split, join) [13]. В результате получаем список - рисунок 6б. По извлечённым для всех пациентов названиям строится словарь лекарств - рисунок 6в (верхнее окно). Встроенные в OpenRefine методы кластеризации позволяют провести ограниченную унификацию элементов словаря - рисунок 6в (нижнее окно). В частности, унифицировать названия лекарств, написанных с прописной и строчной буквы. На последнем шаге (рисунок 6г), список назначенных лекарств нумеруется. В дальнейшем это позволит для каждого лекарства сформировать уникальный идентификатор назначения. Помимо названий лекарств из данных также извлекался основной диагноз. На данном этапе используется инструмент OpenRefine. Результатом выполнения операции в OpenRefine является либо модификация исходных столбцов, либо расширение исходной таблицы. Например, пронумерованный список лекарств по каждому пациенту сохранялся в новом столбце.

\section{5 Связывание извлечённых терминов с понятиями онтологий}

Уровень связывания данных включает два последовательных шага:

1) связывание отдельных понятий с понятиями из собственных и сторонних онтологий;

2) аннотирование данных понятиями собственных онтологий и экспорт конкретной онтологии, т.е. онтологии содержащей конкретные данные.

На этом этапе используется OpenRefine. Инструмент предлагает два механизма для связывания данных. Первый механизм - это механизм автоматического связывания (reconciling). Для его использования в OpenRefine регистрируется внешний источник данных (рисунок 7):

- указывается адрес SPARQL-endpoint (онтология, с понятиями которой будут связываться данные, должна быть доступна по этому адресу); 
- выбираются и/или задаются свойства онтологии, которые будут просматриваться при связывании.
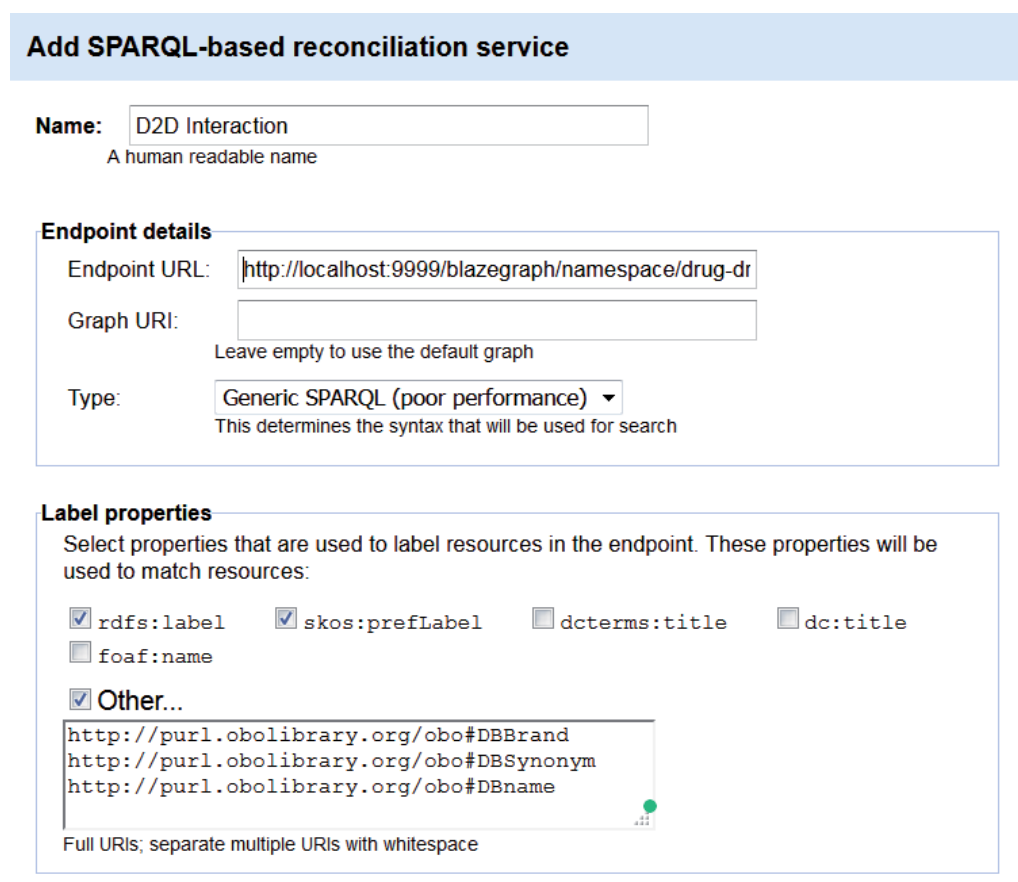

Рисунок 7 - Регистрация внешнего источника данных

Далее для выбранного столбца данных запускается операция автоматического связывания. В ходе этого процесса инструмент пытается подобрать для каждого элемента столбца элемент из онтологии.

На рисунке 8 представлен фрагмент результата связывания названий лекарств, выделенных на предыдущем этапе, и понятий из Онтологии взаимодействия лекарств. Как видно, из трёх лекарств только для двух было найдено понятие в онтологии.

\begin{tabular}{|c|c|c|}
\hline$\nabla$ Medicine name & $\nabla$ Medication english name & $\nabla$ LinkOnD2D \\
\hline Бисопролол & $\begin{array}{l}\text { Bisoprolol } \\
\text { Choose new match }\end{array}$ & $\begin{array}{l}\text { http://purl.obolibrary.org } \\
\text { /obo/CHEBI_3127 }\end{array}$ \\
\hline $\begin{array}{l}\text { Индапамид- } \\
\text { ретард }\end{array}$ & $\begin{array}{l}\text { Indapamide retard } \\
\text { Search for match }\end{array}$ & \\
\hline Валсартан & $\begin{array}{l}\text { valsartan } \\
\text { Choose new match }\end{array}$ & $\begin{array}{l}\text { http://purl.obolibrary.org } \\
\text { /obo/CHEBI_9927 }\end{array}$ \\
\hline
\end{tabular}

Рисунок 8 - Фрагмент результата связывания названий лекарств

Для использования Онтологии взаимодействия лекарств русскоязычные названия извлечённых лекарств (точнее, торговых марок) были переведены на английский язык с помощью онлайн-справочника ${ }^{11}$. Для связывания извлечённых названий лекарств с понятиями онтологии использовались свойства онтологии, которые задают названия действующего вещества (DBname), торговых марок (DBBrand) и синонимы действующих веществ (DBSynonym) - cм. рисунок 7, поле Other. Данный механизм также был использован для связывания выделенных диагнозов с элементами онтологии Международной классификации болезней.

${ }^{11}$ Реестр лекарственных средств России - https://www.rlsnet.ru/ 
Второй механизм позволяет привязывать данные к строго заданным понятиям онтологии. Для этого на основе выделенных терминов составляется SPARQL-запрос к SPARQLendpoint, в которую предварительно загружается онтология. Полученный от сервиса результат в формате JSON-строки (JavaScript Object Notation) сохраняется в отдельном столбце. Встроенными в OpenRefine средствами синтаксического разбора из неё извлекаются нужные данные и помещаются в новый столбец.

На втором шаге данные аннотируются понятиями собственной онтологии. Для этого средствами OpenRefine строится схема (RDF Skeleton) отображения колонок с данными на понятия выбранной онтологии - рисунок 9. Данная схема используется для сохранения данных в формате RDF, иными словами, для аннотированных данных. Каждая строка такой схемы представляет собой тройку: субъект-предикат-объект. Например, в первой строке рисунка 9 указано, что регистрационный номер пациента «Рег.№» представляет собой URI, который через свойство rdf:type связан с классом Patient онтологии H ospital.

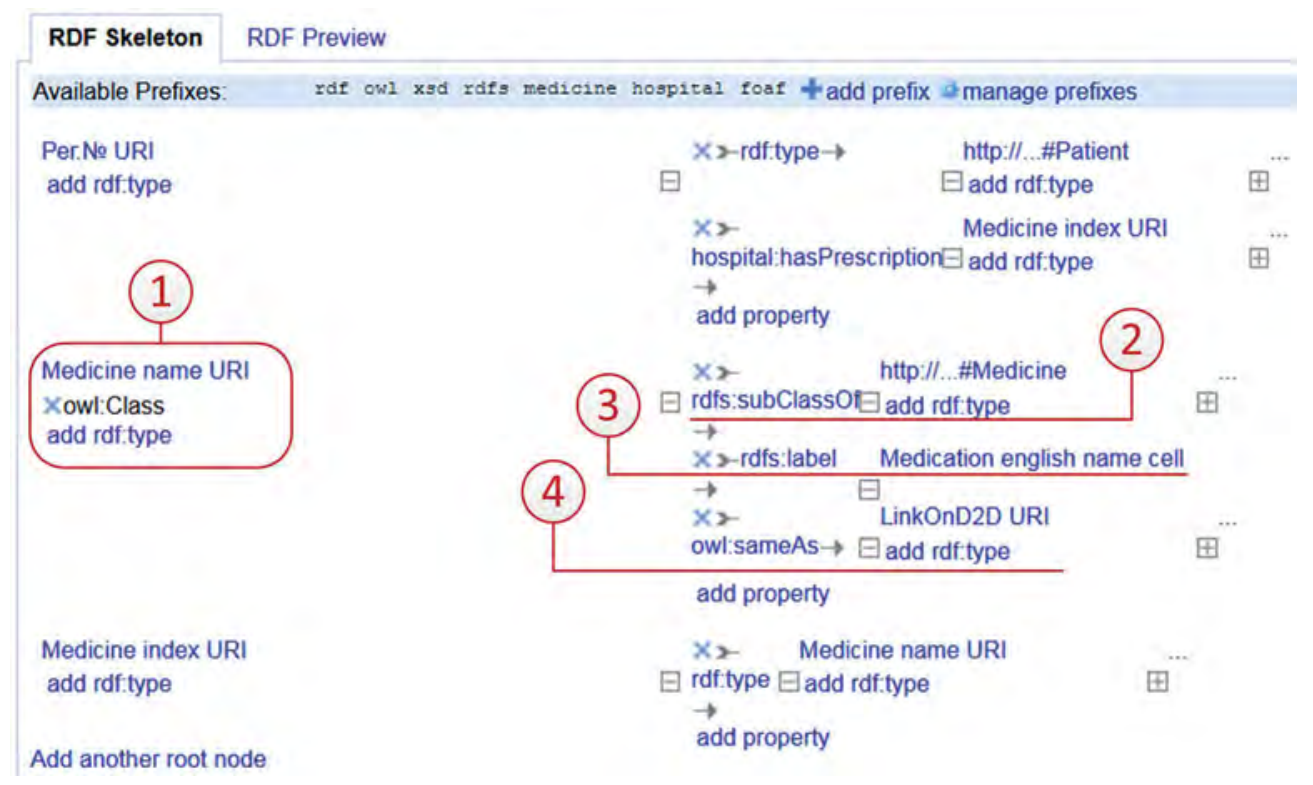

Рисунок 9 - Схема отображения данных на понятия онтологии

Для установления связи с онтологией взаимодействия лекарств формируется три строки схемы отображения (см. рисунок 9). Каждая строка описывает одну тройку. Для всех троек задан один общий субъект - OWL класс, идентификатор для которого строится по русскоязычному названию лекарства (см. рисунок 9-1):

1) В первой строке (рисунок 9-2) задаётся тройка, в которой указывается, что созданный класс лекарства является дочерним классом класса M edicine. Множество таких дочерних классов образует словарь лекарств, сформированный на базе конкретных данных. Словарь включается в метаонтологию M edicineTreatment.

2) Вторая строка задаёт англоязычное название для класса лекарства (рисунок 9-3). Используется отношение rdfs:label.

3) Третьей строкой задаётся тройка, которая привязывает класс лекарства к классам сторонней онтологии через отношение owl:sameAs (рисунок 9-4). Понятия сторонней онтологии после проведения автоматического связывания содержатся в отдельном столбце (см. рисунок 8).

Отображение формирует собственный словарь лекарств, часть элементов которого связана со сторонней онтологией. 
На основе построенной схемы отображения генерируется онтология, которая содержит конкретные данные, проаннотированные понятиями выбранной метаонтологии.

\section{6 Поиск взаимодействующих лекарств}

Возможность поиска обеспечена привязкой назначенных пациенту лекарств к элементам Онтологии взаимодействия лекарств. В последней между отдельными лекарствами установлено отношение «may interact with».

Результат запроса представлен на рисунке 10 в виде графа (регистрационный номер пациента закрашен), построенного с помощью каркаса Metaphactory. Для наглядности граф построен для одного пациента.

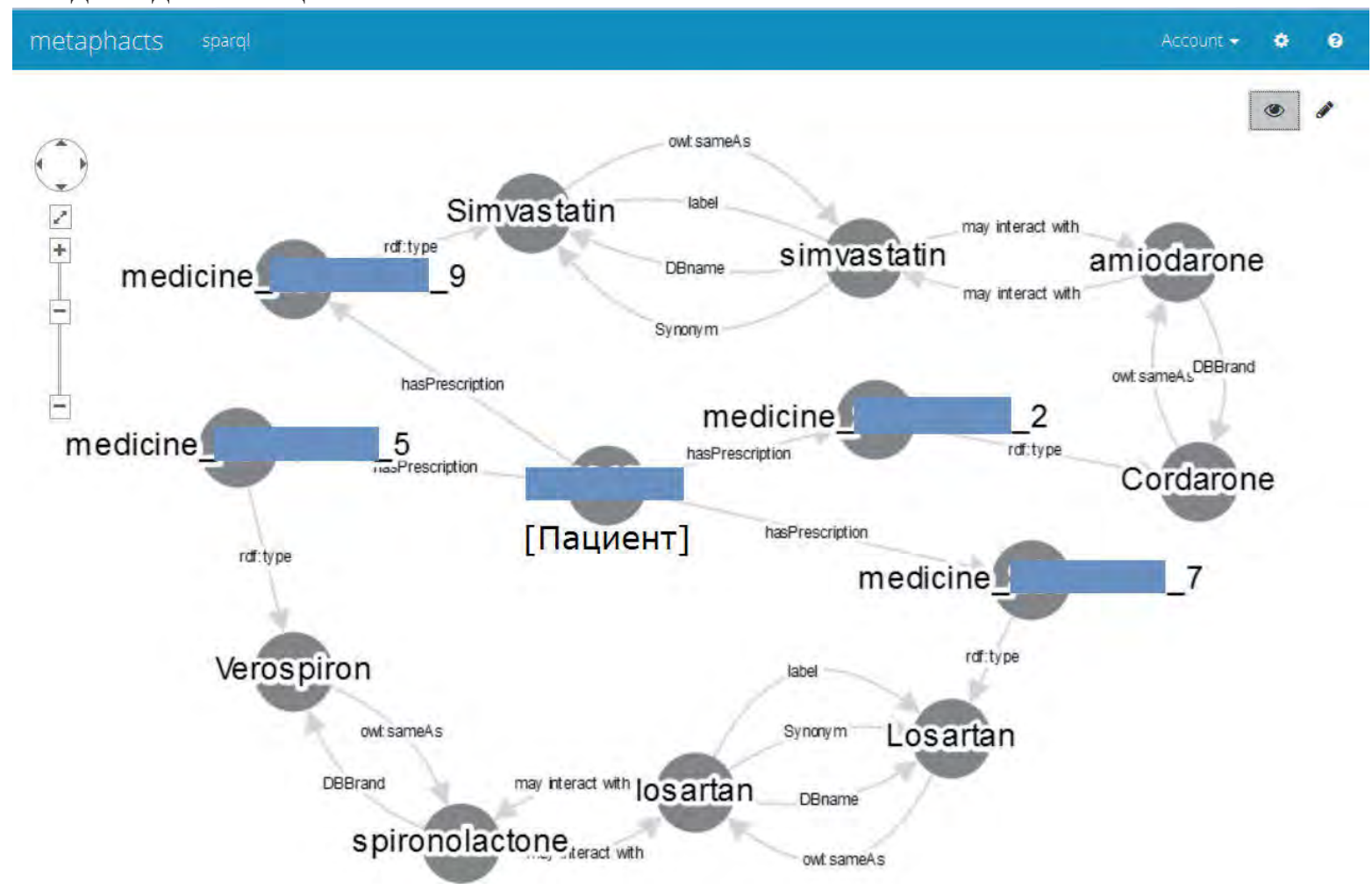

Рисунок 10 - Пример найденного возможного взаимодействия лекарств

Свойством hasPrescription пациент связан с объектом, представляющим собой назначение препарата. Для каждого объекта назначения задан класс лекарственного препарата, взятый из онтологии MedicineTreatment. Класс лекарственного препарата связан свойством owl:sameAs c элементом Онтологии взаимодействия лекарств.

В частности, установлено, что среди прописанных пациенту лекарств могут взаимодействовать две пары: Симвастатин (Simvastatin) - Кордарон (Cordarone) и Верошпирон (Verospiron) - Лозартан (Losartan). Стоит обратить внимание, что взаимодействие Верошпирона (торговая марка) установлено через действующее вещество Спиронолактон (Spironolactone). Как отмечено в п.3.5, это возможно благодаря тому, что в Онтологии взаимодействия лекарств для торговых марок указаны соответствующие действующие вещества.

Для отображения графа использовался Metaphactory. Граф строится с использованием специализированного скриптового языка. Фрагмент кода, описывающий структуру и отображение графа, включает две части:

- SPARQL-запрос для выбора взаимодействующих лекарств, предписанных заданному пациенту; 
- описание, по которому результаты запроса отображаются на элементы графического интерфейса.

\section{7 Представление данных пользователю}

Кроме представленного в предыдущем разделе графа, возможны и иные графические представления. Например, на рисунке 11 представлена таблица пациентов, где каждому пациенту сопоставлен диагноз из Международного классификатора болезней.

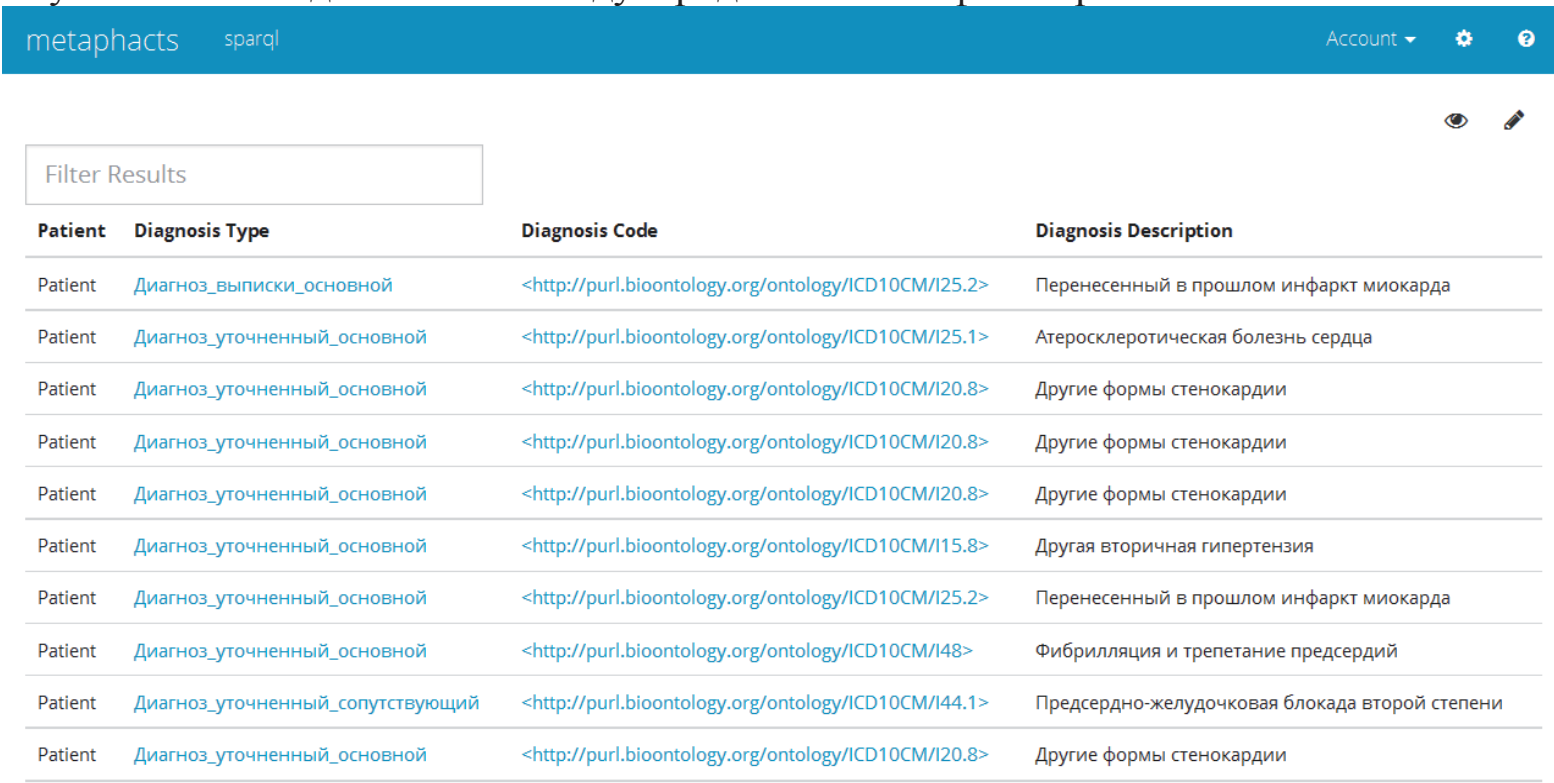

Рисунок 11 - Таблица диагнозов пациентов

На рисунке 12 представлена сводная диаграмма, отражающая частоту назначения отдельных лекарств в рамках отдельного учреждения (в объеме предоставленных данных).
metaphacts sparal
Account $\%$ ○

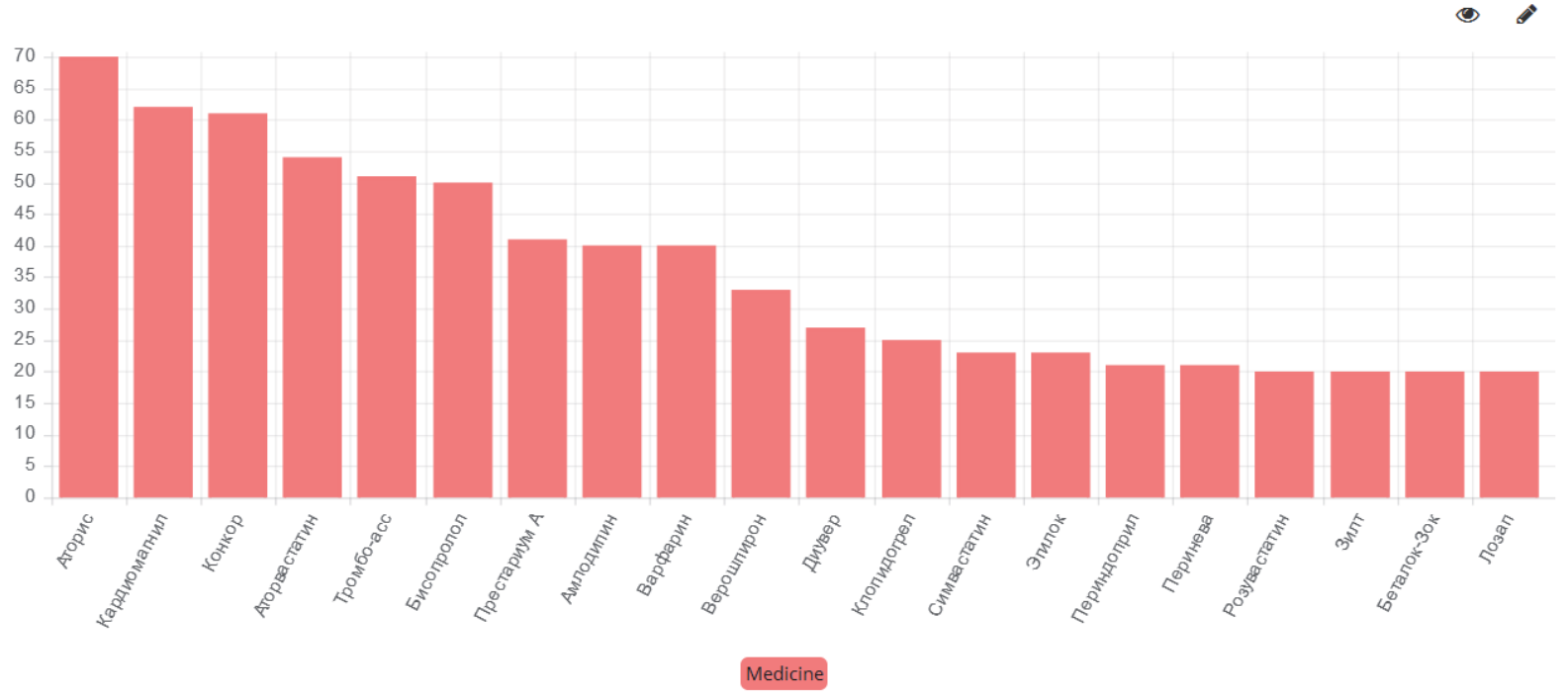

Рисунок 12 - Диаграмма частоты назначения лекарств 


\section{Заключение}

Представлен подход к совместному использованию онтологий и JDL модели слияния данных. Подход проиллюстрирован на примере сценария поиска взаимодействующих лекарств. Онтологии позволили: формально описать фрагмент ПрО; аннотировать в терминах ПрО медицинские данные; связать данные между собой и со сторонними онтологиями; получить на основе связанных данных дополнительную информацию - пары взаимодействующих лекарств. Модель слияния данных использована в качестве общей схемы, определяющей уровни процесса обработки и их взаимосвязь.

Даны источники открытых медицинских онтологий и представлены варианты использования некоторых из них. Описаны варианты использования инструментов для подготовки данных и онтологий, хранения и отображения онтологий.

При реализации сценария выявлен ряд затруднений. Значительных усилий потребовало выделение названий лекарств, их унификация и перевод на английский язык. Использовать английские названия необходимо для совместимости со сторонними онтологиями. Кроме того, использование русскоязычных идентификаторов онтологических сущностей приводило к ошибкам в работе используемых инструментов.

Развитие представленного сценария может пойти по нескольким направлениям: автоматизация процесса слияния данных, анализ и включение дополнительных сторонних медицинских онтологий, расширение представленного сценария.

Предложенный подход является перспективной основой для реализации процессов слияния данных и знаний в рамках МИС.

\section{Список источников}

[1] Лушнов, А.M. Медицинские информационные системы: многомерный анализ медицинских и экологических данных / А.М. Лушнов, М.С. Лушнов. - Санкт-Петербург, «Геликон Плюс», 2013 - 460 с.

[2] F oo P.H ., Ng G.W. High-level Information Fusion: An Overview // J. Adv. Inf. Fusion. - 2013. - Vol. 8. - №. 1. C. 33-72.

[3] Steinberg A.N., B owman C.L. Revisions to the JDL Data Fusion Model / Llinas J., Hall D.L., Liggins M.E. (ed.). Handbook of Multisensor Data Fusion: Theory and Practice. - CRC Press, 2009. - P. 44-67.

[4] B owman C.L., Steinberg A.N. Systems Engineering Approach for Implementing Data Fusion Systems / Llinas J., Hall D. L., Liggins M. E. (ed.). Handbook of Multisensor Data Fusion: Theory and Practice. - CRC Press, 2009. P. 561-596.

[5] Lushnov, M. Medical knowledge representation for evaluation of patient's state using complex indicators / M. Lushnov, V. Kudashov, A. Vodyaho, M. Lapaev, N. Zhukova, D. Korobov // International Conference on Knowledge Engineering and the Semantic Web. - Springer International Publishing, 2016. - P. 344-359.

[6] Rodrigues, J J. (ed.) Health Information Systems: Concepts, Methodologies, Tools and Applications / J.J. Rodrigues, B. Vaidya // Health Information Systems: Concepts, Methodologies, Tools and Applications - IGI Global, 2009. - Vol.1.

[7] Parry, D. Healthcare Information Systems and the Semantic Web / D. Parry // Health Information Systems: Concepts, Methodologies, Tools and Applications - IGI Global, 2009. - Vol. 1. - P. 178-184.

[8] Simonet, M. Ontologies in the Health Field / M. Simonet, R. Messai, G. Diallo, A. Simonet // Health Information Systems: Concepts, Methodologies, Tools and Applications - IGI Global, 2009. - Vol. 1. - P. 185-203.

[9] Lushnov, M. Medical text processing for SMDA project / M. Lushnov, T. Safin, M. Lapaev, N. Zhukova // CEUR Workshop Proceedings. 2016. - Vol. 1613. - P. 1-13.

[10] Poulymenopoulou, M. A LOD-Based Service for Extracting Linked Open Emergency Healthcare Data / M. Poulymenopoulou, F. Malamateniou, G. Vassilacopoulos // International Conference on Bioinformatics and Biomedical Engineering. - Springer International Publishing, 2015. - P. 85-92.

[11] Клещев, А.C. Парадигма автоматизации интеллектуальной профессиональной деятельности. Часть 1. Особенности интеллектуальной профессиональной деятельности / А.С. Клещев, М.Ю. Черняховская, Е.А. Шалфеева // Онтология проектирования. 2013. №3(9). - С. 53-69. 
[12] Клещев, $\boldsymbol{A . C .}$ Парадигма автоматизации интеллектуальной профессиональной деятельности. Часть 2. Автоматизация отрасли / А.С. Клещев, М.Ю. Черняховская, Е.А. Шалфеева // Онтология проектирования. 2013. №4(10). - C. 28-41.

[13] Verborgh, V. Widle, M. D. Using OpenRefine. -Birmingham, Packt Publishing Ltd., 2013 - 114 p.

\title{
ONTOLOGY-DRIVEN APPROACH TO MEDICAL DATA FUSION
}

\author{
S.V. Lebedev ${ }^{1}$, N.A. Zhukova ${ }^{2}$ \\ ${ }^{1}$ Saint Petersburg Electrotechnical U niversity "LETI", Saint P etersburg, Russia \\ lebedev.sv.etu@gmail.com \\ ${ }^{2}$ ITM $O$ U niversity, Saint Petersburg, Russia \\ nazhukova@mail.ru
}

\begin{abstract}
A large volume of operational and statistical data has been stored within medical information system databases. The stored data represents a loosely-coupled and semi-structured clump. To increase information support of medical procedures it is necessary to implement data linking processes into the medical information systems. The aforementioned processes should be able to collect data from diverse sources and produce a linked view that can be used for medical evaluation, planning, etc. To solve this problem, an approach based on JDL (Joint Directors of Laboratories) data fusion model and ontologies is presented. JDL model defines functional levels of data fusion and their interconnections. Ontologies are used to set links between data and knowledge. To specify and evaluate the proposed approach, a simplified scenario is constructed. The scenario was prepared for the Federal Almazov North-West Medical Research Center. The proposed scenario includes the following actions: extraction of a list of drugs and diagnosis from semi-structured data; annotation of extracted data using author's ontology and third-party ontologies; revealing interacting drugs that are prescribed to a patient; data representation. The scenario shows that ontology-driven data fusion approach has a great potential for linking data and knowledge within medical information systems. It helps to detail each level of data fusion, reveal problems that need further research and describe use cases for Semantic Web instruments.
\end{abstract}

Key words: data fusion model, ontology, medical information system, Linked O pen Data.

Citation: Lebedev SV, Zhukova NA. Ontology-driven approach to medical data fusion. Ontology of designing, 2017; 7(2): 145-159. DOI: 10.18287/2223-9537-2017-7-2-145-159.

\section{References}

[1] L ushnov AM, L ushnov MS. Medical Information Systems: multidimensional medical and ecological data analysis [In Russian] - Saint-Petersburg, «Helicon Plus», 2013 - 460 pp.

[2] F oo PH, Ng G W. High-level Information Fusion: An Overview. J. Adv. Inf. Fusion. - 2013. - Vol. 8 (1). - P 3372.

[3] Steinberg AN, B owman CL. Revisions to the JDL Data Fusion Model. Llinas J., Hall D. L., Liggins M. E. (ed.). Handbook of Multisensor Data Fusion: Theory and Practice. - CRC Press, 2009. - P. 44-67.

[4] B owman CL, Steinberg AN. Systems Engineering Approach for Implementing Data Fusion Systems. Llinas J., Hall D. L., Liggins M. E. (ed.). Handbook of Multisensor Data Fusion: Theory and Practice. - CRC Press, 2009. P. 561-596.

[5] L ushnov M, Kudashov V, Vodyaho A, Lapaev M, Zhukova N, Korobov D. Medical knowledge representation for evaluation of patient's state using complex indicators. International Conference on Knowledge Engineering and the Semantic Web. - Springer International Publishing, 2016. - P. 344-359.

[6] Rodrigues JJ (ed.), Vaidya B. Health Information Systems: Concepts, Methodologies, Tools and Applications IGI Global, 2009. - Vol.1.

[7] Parry D. Healthcare Information Systems and the Semantic Web. Health Information Systems: Concepts, Methodologies, Tools and Applications - IGI Global, 2009. - Vol. 1. - P. 178-184. 
[8] Simonet M, Simonet M, Messai R, Diallo G, Simonet A. Ontologies in the Health Field. Health Information Systems: Concepts, Methodologies, Tools and Applications - IGI Global, 2009. - Vol. 1. - P. 185-203.

[9] Lushnov M, Safin T, Lapaev M, Zhukova N. Medical text processing for SMDA project. CEUR Workshop Proceedings. 2016. - Vol. 1613. - P. 1-13.

[10] Poulymenopoulou $\boldsymbol{M}$, Malamateniou F, Vassilacopoulos G. A LOD-Based Service for Extracting Linked Open Emergency Healthcare Data. International Conference on Bioinformatics and Biomedical Engineering. - Springer International Publishing, 2015. - P. 85-92.

[11] Kleshhev A.S., Chernyahovskaya E .A., Shalfeeva E .A. Paradigm of intellectual professional activity automation. Part 1. Features of intellectual professional activity [In Russian]. Ontology of designing. - No. 3(9). - 2013. - pp. 63-69.

[12] Kleshhev A.S., Chernyahovskaya E .A., Shalfeeva E .A. Paradigm of intellectual professional activity automation. Part 2. Features of intellectual professional activity [In Russian]. Ontology of designing. - No. 4(10). - 2013. - pp. 28-41.

[13] Verborgh, V. Widle, M. D. Using OpenRefine. -Birmingham, Packt Publishing Ltd., 2013 - 114 p.

\section{Сведения об авторах}

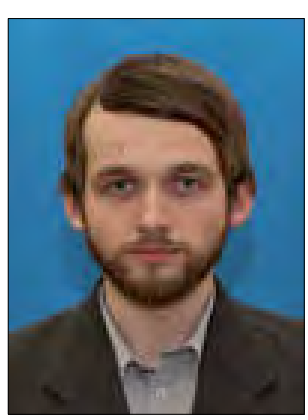

Лебедев Сергей Вячеславович, 1984 г. рождения. Окончил Санкт-Петербургский государственный электротехнический университет «ЛЭТИ» им. В.И. Ульянова (Ленина) (СПбГЭТУ ЛЭТИ) в 2010 году. Ассистент кафедры вычислительной техники СПбГЭТУ ЛЭТИ. В списке научных трудов более 10 работ в области многоагентных систем и технологий семантического Web.

Lebedev Sergey Vyacheslavovich (b. 1984) graduated from the Saint Petersburg Electrotechnical University "LETI" (ETU) in 2010. He is an assistant at ETU (Department of Computer Science and Engineering). He is co-author of more than 10 publications in the field of multiagent systems and semantic web technologies.

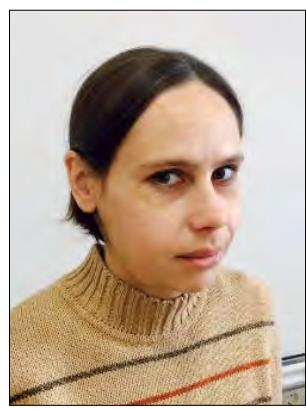

Жукова Наталья Александровна, 1983 г. рождения. Окончила Санкт-Петербургский государственный электротехнический университет «ЛЭТИ» им. В.И. Ульянова (Ленина) (СПбГЭТУ ЛЭТИ) в 2006 году, к.т.н. (2008). Доцент кафедры математического обеспечения ЭВМ СПбГЭТУ ЛЭТИ и кафедры информатики и прикладной математики СанктПетербургского национального исследовательского университета информационных технологий, механики и оптики. В списке научных трудов более 20 работ в области обработки и анализа данных.

Zhukova Nataly Alexandrovna (b. 1983) graduated from the Saint Petersburg Electrotechnical University "LETI" (ETU) in 2006. Ph.D (2008), Associate Professor, ETU and ITMO University. She is co-author of more than 20 publications in the field of data processing and analyses. 


\title{
УДК 001.812
}

\section{ИНФОРМАЦИОННАЯ МОДЕЛЬ РЕЗУЛЬТАТОВ НАУЧНОГО ИССЛЕДОВАНИЯ}

\author{
А.М. Беглер \\ Санкт-Петербургский государственный университет, Санкт-Петербург, Россия \\ alena.begler@gmail.com
}

\begin{abstract}
Аннотация
Результаты научного исследования существуют в разных формах, появляются на разных этапах процесса исследования и описываются несколькими относящимися к ним понятиями. Во-первых, это этапы исследования, на которых они производятся. Выделяют несколько этапов - от поиска информации до распространения результатов. На каждом из них исследователь осуществляет деятельность, направленную на получение результата. Во-вторых, сами результаты исследования существуют в разных формах - от традиционных статей до наборов данных, получаемых в результате экспериментов или знаний исследователя, лежащих в основе гипотез. Их условно можно разделить на контент, данные и знания. Для производства результатов, в-третьих, используется набор инструментов, которые могут быть классифицированы по результату, который они обеспечивают. В-четвёртых, результатам может быть обеспечен разный уровень доступности, который складывается из степени их открытости научному сообществу и интегрированности в существующие системы организации исследовательской информации. В работе впервые предлагается информационная модель результатов исследования, соединяющая рассмотренные характеристики. Изложенный материал может быть полезен при организации научно-исследовательских работ, в особенности на уровне руководителей и администраторов научных групп.
\end{abstract}

Ключевые слова: результаты научного исследования, продукты исследования, жизненный ичил исследования, этапы исследования, инструменты исследования, организация исследования.

Цитирование: Беглер, А.М. Информационная модель результатов научного исследования / A.М. Беглер // Онтология проектирования. - 2017. - Т. 7, №2(24). - С. 160-171. - DOI: 10.18287/2223-9537-2017-7-2-160-171.

\section{Введение}

Основной результат научного исследования - увеличение знания об исследуемой области. Это знание существует в разных формах: оно описано в статьях, лежит в основе гипотез исследования, определяет используемые экспериментальные методы и так далее. Перечисленные формы результатов можно назвать продуктами научного исследования (scholarly products). Они представлены, по большей части, в цифровом виде, и их количество быстро растёт [1]. При этом доступность полученных ранее результатов со временем снижается [2].

Проблема снижения доступности результатов исследований при возрастании их общего количества отчасти решается появлением специализированного инструментального обеспечения. Например, сейчас функционирует более полутора тысяч хранилищ исследовательских данных и несколько десятков систем организации информации для лабораторий и научноисследовательских организаций. Существующие решения хотя и обеспечивают сохранение продуктов научного исследования, но обладают двумя недостатками. Во-первых, они не универсальны и решают определённые частные проблемы. Во-вторых, они не позволяют определить, организация какой части результатов страдает. Так, многие инструменты и системы позволяют сохранить данные, получаемые в результате экспериментов, но не всегда 
позволяют обозначить их взаимосвязи с другими продуктами исследования (например, статьями) и сохранить связанные с ними знания исследователей (например, о процессе сбора этих данных).

Решение обеих проблем могла бы обеспечить базовая, не привязанная к определённой предметной области (ПрО), модель продуктов научного исследования. Такая модель эксплицитно обозначит весь спектр имеющихся продуктов исследования, их взаимосвязи и характеристики. Подобный подход предлагается в работах [3,4] для конструирования порталов научных знаний. Они используют базовые онтологии научной деятельности и научного знания, которые лежат в основе портала и могут быть расширены за счёт онтологий ПрО.

В работе предлагается базовая информационная модель результатов научного исследования, описывающая, что именно получается в результате исследования (продукт исследования), каким образом (на каком этапе) и с помощью чего (инструмент).

Предлагаемая модель позволит представить структуру продуктов научного исследования во всей полноте и выстроить единую и расширяемую систему, которая может быть использована несколькими способами.

1) Для организации информационных потоков исследования, в первую очередь - для руководителей и администраторов научных групп. При таком использовании она может выступать в качестве «каркаса», на котором будет построена система организации конкретной научной группы.

2) При подготовке инструментов для обеспечения научной информации - для выявления потребностей в инструментальном обеспечении.

3) При конструировании баз и порталов знаний ПрО. В этом случае она должна быть дополнена онтологиями продуктов исследования и инструментария ПрО.

Применение предлагаемой модели описано в заключении к статье. В четырёх разделах основной части статьи представлена каждая из входящих в модель характеристик продукта научного исследования: в первом кратко рассматривается процесс производства продукта исследования; во втором - виды продуктов; в третьем - их доступность, в четвёртом - инструментальное обеспечение. В каждом разделе приведён конкретный способ применения каждой из частей модели на примере подготовки текста научной статьи. Для большинства используемых терминов в скобках даны англоязычные аналоги.

\section{1 Процесс научного исследования}

Существует несколько классификаций научно-исследовательского процесса (research lifecycle, scientific workflow) по этапам. Kramer и Bosman [5] выделяют шесть этапов научного исследования: поиск информации, анализ, написание текста, публикация, распространение и оценка. Kubilius [6] предлагает цикл исследовательских работ, характерных для наук о поведении и нейронаук: формулирование гипотезы, разработка программы эксперимента, сбор данных, анализ данных, подготовка рукописи, публикация материала. Nicholas и Rowlands [7] выделяют восемь стадий жизненного цикла исследования и соотносят их с использованием разных социальных медиа: определение возможностей для исследования, научное сотрудничество, получение финансирования, обзор литературы, сбор данных, анализ данных, распространение результатов, менеджмент процесса исследования.

Если рассматривать только этапы, относящиеся к производству непосредственно продукта научного исследования, и не брать в расчёт обеспечение работ, то видно, что описанные классификации совпадают в нескольких ключевых пунктах (рисунок 1). Сначала происходит поиск информации по теме исследования (например, анализ литературы), в результате которого оценивается уже имеющаяся информация и формулируется гипотеза исследования. По- 
сле этого начинается сбор и анализ данных (в том числе планирование эксперимента), с помощью которого сформулированная гипотеза проверяется. Когда данные проанализированы, происходит подготовка текста, в котором полученная новая информация сформулирована в явной форме. Далее происходит публикация материалов исследования (не только статей по результатам, но и, например, массивов данных). Оценка эффекта от публикации (комментарии, цитирования) помогает исследователям оценить, насколько актуальна полученная информация среди коллег и общественности. К условно завершающему этапу, который в описанных классификациях назван «Распространение», добавлена «Интеграция»: распространение ориентировано на общественность и коллег - посты в социальных сетях (в том числе профессиональных) и блогах, интервью, выступления с лекциями и так далее, а интеграция касается скорее внедрения новой информации в существующее информационное поле, например, добавление в Википедию или тематические проекты, пополнение баз данных и знаний по изучаемому феномену.

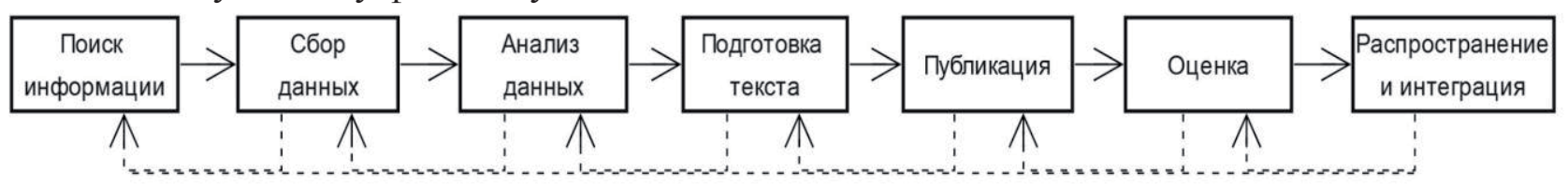

Рисунок 1 - Этапы научного исследования

Отдельное исследование проходит через описанные этапы не линейно, а скорее итеративно. Например, неожиданный результат анализа данных может повлечь за собой сбор новых данных или дополнительный анализ литературы, а подготовка текста - идти параллельно со сбором и анализом данных.

Пример. Текст научной статьи готовится на этапе «Подготовка текста», но с ним связана часть продуктов, полученных на предшествующих этапах. Например, найденные на этапе поиска информации статьи могут быть процитированы в тексте. Зная это, можно подобрать инструмент (менеджер библиографии), который позволит не только сохранять тексть статей, но и в дальнейшем автоматически вставлять циттрования и формировать библиографию. Непосредственно на этапе подготовки текста стоит принимать во внимание последующие этапь. Например, то, в каком издании будет опубликован текст (этап «Публикация») повлияет как на его структуру, так и на особенности содержания. Такие связи существуют между всеми этапами, и заблаговременное их построение экономит время (текст претерпит меньще изменений в процессе публикации) и препятствует потерям информации (сохранённые тексты статей не будут потеряны).

Каждому из этапов присуща деятельность, направленная на производство продукта исследования (рисунок 2).

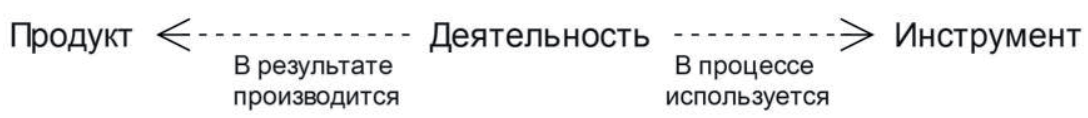

Рисунок 2 - Взаимосвязь деятельности, инструментов и продуктов исследования

Сделан ряд попыток обобщения отдельных направлений исследовательской деятельности. Unsworth [8] предлагает несколько базовых элементов научной деятельности (scholarly primitives): изучение, аннотирование, сравнение, проставление ссылок, измерение, пояснение и представление. Palmer с соавторами [9] расширяют количество базовых элементов и на их основе выделяют несколько форм деятельности, относящихся к работе с информацией (scholarly information activities) - они, как и базовые элементы, междисциплинарны и могут происходить на любом из описанных этапов. Эти виды деятельности распадаются на пять ключевых групп (рисунок 3): поиск, сбор, чтение, написание и взаимодействие.

Пример. При подготовке текста происходит поиск литературы (поиск), подготовка ссылок на источники (сбор), чтение найденных текстов (чтение), собственно подготовка текста (написание) и обсуждение с 
соавторами (взаимодействие). Инструментальное обеспечение исследования должно предусматривать все описанные виды деятельности и получаемые в их результате продукты.

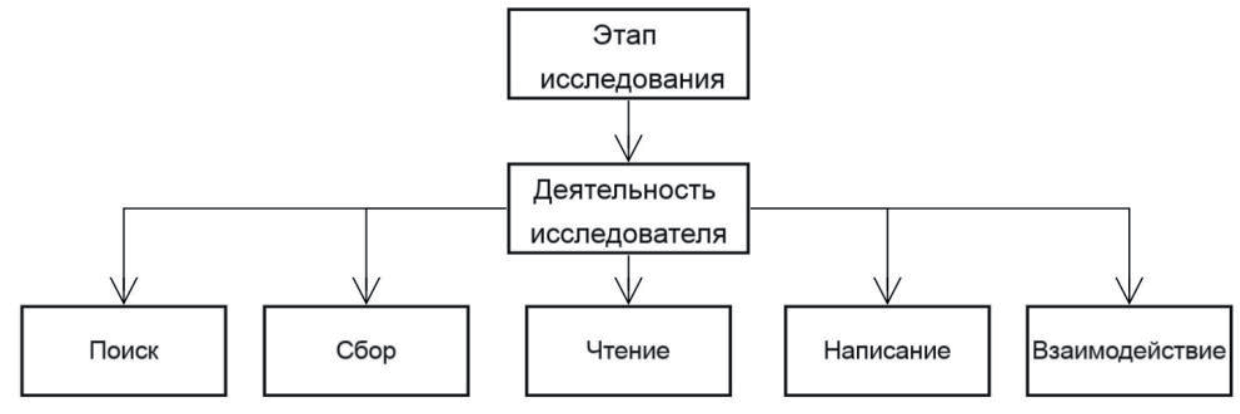

Рисунок 3 - Виды деятельности исследователя

\section{2 Формы продуктов исследования}

Если раньше продуктами исследования считались в основном научные статьи, то сейчас к их числу относятся также массивы данных, экспериментальные программы, протоколы исследований и т.п. Косвенно об изменениях продукта исследования можно судить по предлагаемым «альтернативным метрикам» (altmetrics) оценки научного влияния, в числе которых указываются цитирования в Википедии, добавления в менеджер библиографии, загрузки pdfфайлов, лайки и репосты в социальных сетях, упоминания в популярных научных блогах, комментарии на страницах публикаций и др. [10,11].

О расширении спектра продуктов научного исследования свидетельствует и ряд изменений в политике научных фондов, издателей и производителей инструментов:

- начиная с 2013 года Национальный научный фонд (National Scientific Foundation, NSF) запрашивает информацию от руководителей проектов не только о статьях, но и других продуктах исследований [12];

- Elsevier, один из крупнейших научных издателей, отмечает важность открытия доступа и организации научных данных [13];

- популярная социальная сеть для исследователей ResearchGate присваивает пользователям рейтинг, основанный, в числе прочего, на количестве положительно оценённых сообществом ответов на запросы других исследователей.

Продукты, описанные в примерах, отличаются ещё и по виду - в первых двух случаях речь идёт о данных и контенте, в третьем - о знаниях. Продукт исследования не гомогенен и отличается как на разных этапах исследования, так и внутри одного этапа. Контент понимается как слабоструктурированная информация, данные - отдельные упорядоченные факты, знания - результат интерпретации данных или метаданные (данные о данных). Результатом поиска информации на уровне контента может стать подборка релевантных статей; на уровне данных - сведённые воедино факты по интересующей тематике, выделенные из собранного контента; на уровне знаний - сформулированная гипотеза исследования (выражающая как знания исследователя об области, так и потребность в их увеличении) [14].

После формулировки гипотезы появляется возможность перейти к этапу сбора данных продумать план эксперимента, его материальное выражение - протокол исследования, результатом проведения которого будут необходимые «сырые» данные, а также экспериментальные программы и другие составные части эксперимента.

Полученные данные обрабатываются с помощью программ анализа, и в результате получаются численные выражения следствий выдвинутых гипотез - размеры эффектов и корре- 
ляций, которые подлежат интерпретации. Полученные результаты оформляются в виде визуализаций (графиков, таблиц, схем) и вместе с описанием формируют подготовленный текст, который сопровождается техническими файлами, например, шаблонами оформления или изображениями.

На этапе публикации текст и другие материалы, например данные и код, отправляются в тематические журналы и репозитории и проходят процесс рецензирования, в процессе которого происходит коммуникация с редакторами и рецензентами. После публикации необходимо провести оценку: отследить отдельные упоминания - например, добавления в списки для чтения других исследователей, классические цитирования и т.п., - которые могут сформировать список цитирований и увеличить знания исследователя о восприятии проведённого исследования коллегами.

На этапе распространения и интеграции исследователь может выложить в тематические репозитории отдельные части своего исследования, или дополнительно описать их, или добавить полученные знания, например, в Википедию, в другие тематические проекты.

Обобщённо данный подход представлен на рисунке 4. Обратим внимание, что на большинстве этапов процесс получения итогового продукта идёт от контента к знаниям (получение знания - цель этих этапов); на этапах же сбора данных и оценки влияния - наоборот, отправной точкой становятся знания, а цель этих этапов - накопление данных.

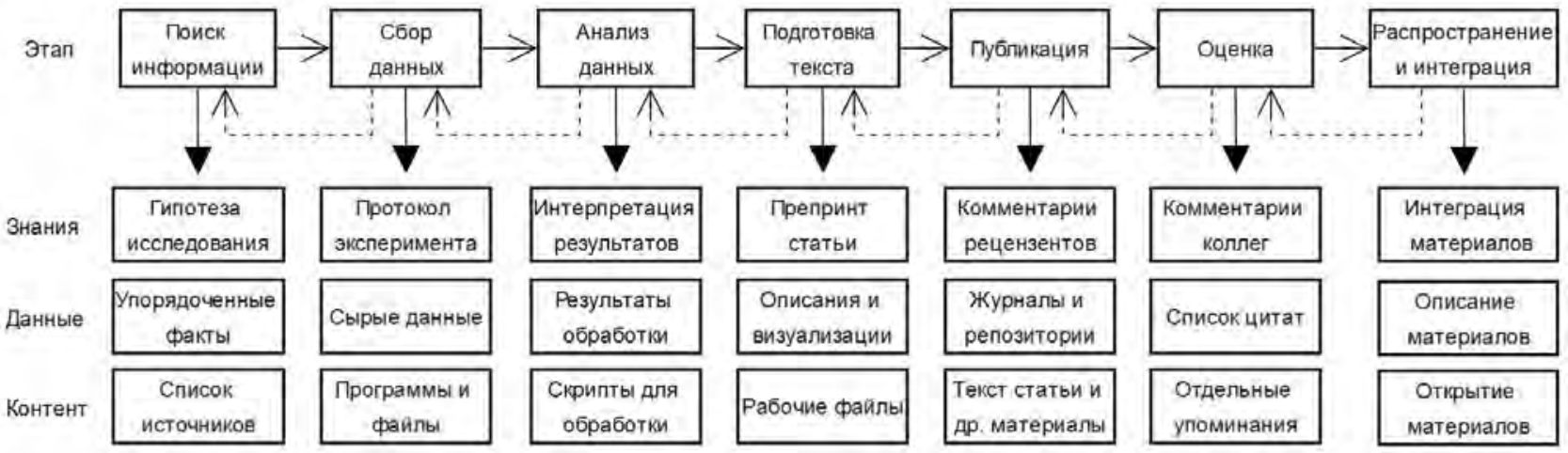

Рисунок 4 - Анализ продукта научного исследования с позиции его разделения на контент, данные и знания

Разделение на данные, знания и контент при этом достаточно условно и необходимо, в первую очередь, для охвата всего спектра продуктов исследования. Если рассматривать основной «физический» продукт этапа как данные, то контент - это те куски, из которых он получился, а знания - это метаданные о процессе и результате его получения. Ценность такого разделения - в экспликации всех продуктов исследования и включении их в явном виде в организационную часть исследования. Не все продукты обязательно должны сохраняться в процессе проведения исследования, но данный подход позволяет принять более обоснованные решение о такой необходимости.

Наиболее часто, когда говорят о результате исследования, речь идёт о данных и, в меньшей степени, о контенте. Так, например, специализированные хранилища данных созданы в первую очередь для контента и данных (материалов для подготовки экспериментов, массивов сырых данных и т.д.), однако добавленные в некоторые инструменты вики-системы и категории делают их пригодными и для работы со знаниями.

Пример. Кроме собственно текста статьи, являющегося основным продуктом на этапе «Подготовка текста», появляются также список источников и «выжимки» из них, схемы и таблиць, результаты обработки данных, информация о вкладе соавторов, идеи о дальнейшем направлении исследований. Первые три можно выявить, акиентируя внимание на то, из чего состоит текст (это контент, связанный с этим этапом), последние два отвечают на вопрос «что мы знаем об этом тексте?» - это знания данного этапа. Эти 
продукты должны учитываться в прочессе исследования - «выжсимки» из источников сохраняться и фиксироваться, таблицы и схемы - сущцествовать в оригинальных форматах (для возможности изменения и последующего использования) и т.д.

\section{3 Доступность продукта исследования}

Важная характеристика продукта исследования - уровень его доступности для других исследователей. Науку можно назвать «экономикой дара» (gift economy), в которой ценность исследования определяется во многом тем, насколько свободно доступны его результаты другим исследователям [15]. Свидетельством важности этого аспекта продукта исследования можно считать увеличение продуктов исследований, находящихся в открытом доступе. Например, количество таких статей возросло с менее чем двадцати тысяч в 2000-м году до почти двухсот тысяч к 2009-му [16], достигнув, таким образом, примерно двадцати процентов от общего количества статей [17] и продолжало расти, составляя к 2014 году уже около 24 процентов [18].

Доступность определяется двумя характеристиками: открытостью и интегрированностью. Открытым продуктом может воспользоваться каждый (обычно он распространяется под открытыми лицензиями). Приватный продукт - тот, который доступен только исследовательской группе и, возможно, организации (либо вообще не выложен в сеть и хранится, например, в частном хранилище, или выложен под частной лицензией). Открытость способствует распространению продукта исследования, но для полноценного распространения продукта недостаточно открыть к нему доступ - он должен находиться там, где потенциальный пользователь сможет его найти, то есть, быть не только открытым, но и интегрированным в информационное поле соответствующей области исследований. Например, массив данных исследования, доступный для скачивания только с сайта научной группы, будет хотя и открытым, но не интегрированным. Тогда как тот же массив, хранящийся под открытой лицензией в тематическом хранилище (например, figshare) - уже и открытый, и интегрированный. Основной критерий отнесения продукта к интегрированным - возможность доступа к нему посредством обращения к общепринятым поисковикам и хранилищам.

И открытость продукта, и его интеграция определяются возможностями инструментов работы с продуктом исследования, о которых речь пойдёт в следующей части. Поэтому при подборе инструментов нужно учитывать план по распространению продукта, фиксирующий точку зрения на аспекты открытости и интегрированности продуктов исследования.

Пример. Открытость текста статьи отчасти обеспечивается публикацией. Интегрированности способствует индексирование в Web of Science или Scopиs. Интегрированность других продуктов, связанных с текстом, например, отдельных результатов обработки данных, зависит от исследовательской области например, в области функциональной магнитно-резонансной томографии существует несколько проектов, предназначенных для интеграции полученньх результатов сканирования головного мозга (ВraіпМар, Neurosynth, NeuroVault).

\section{4 Инструменты для работы с продуктом исследования}

Интернет-ресурсы для исследователей появились в начале 70-х годов [19], а уже через 30 лет сеть Интернет для научной коммуникации используют практически все исследователи [20]. В цифровое пространство переносятся практически все этапы исследовательского процесса: от общения с коллегами (через почтовые агенты и сервисы видеосвязи) до получения данных (например, проведение экспериментов или привлечение участников с помощью онлайновых сервисов) и доступа к опубликованным материалам [21]. К настоящему времени существует несколько сотен различных инструментов для обеспечения научного исследования, большинство из которых созданы специально для учёных, а не адаптированы ими [22]. 
Исследования инструментального обеспечения в основном имеют характер анализа средств научной коммуникации (scholarly communications). Термин «коммуникация» при этом понимается максимально широко - как любая передача информации, поэтому охватывает практически все возможные инструменты, используемые в исследованиях.

Можно выделить несколько десятков видов инструментов, которые используются в процессе работы над продуктом научного исследования (в скобках даны примеры):

- базы и поисковики статей (РИНЦ, Google Scholar, Scopus);

- сервисы рекомендаций (F1000Prime);

- инструменты доступа к литературе (Open Access Button);

- сервисы аннотирования и чтения (Hypothesis, ReadCube);

- инструменты для доступа к протоколу (плану) исследования (myExperiment, protocols.io);

- графические и видео редакторы (Photoshop, Inkscape);

- программы для демонстрации экспериментов (PsychoPy, Presentation);

- онлайн-опросы (Google Forms, SurveyMonkey);

- оффлайновые экспериментальные методики;

- программы для обработки данных (R, SPSS);

- инструменты визуализации данных (Plotly);

- редакторы исходного кода и интегрированные среды разработки (Notepad++, RStudio, PyCharm);

- инструменты совместного авторства (Google Docs, Authorea);

- менеджеры библиографий (Mendeley, Papers);

- предпубликационная оценка (egap, OSF Preregistration);

- инструменты для подбора журнала (SCImago, Elsevier Journal Finder, Jane);

- инструменты публикации (doi, PeerJ, лицензии);

- послепубликационная оценка (PubPeer);

- инструменты отслеживания цитирований (Altmetrics, JCR, Scopus);

- исследовательские профили (ResearchGate, ORCID);

- блоги и микроблоги (Twitter);

- тематические каналы коммуникации (сообщества, списки рассылок);

- публичные хранилища (figshare, Open Science Framework, F1000Posters, arXiv.org);

- тематические базы знаний, например, описания методик и экспериментальных процедур;

- онтологии (An ontology of scientific experiments - EXPO, Mental Functioning Ontology MFO, Cognitive Paradigm Ontology - CogPO).

Эти инструменты используются на разных этапах исследования, предназначены для определённой формы продукта исследования и могут обеспечивать разные уровни его доступности.

Наиболее часто инструменты классифицируются по этапам исследования, на которых они используются (рисунок 5), при этом этапы распространения и интеграции разведены. Такая классификация достаточно условна. С одной стороны, на одном этапе научного исследования могут использоваться инструменты из нескольких групп (например, при написании текста это инструменты совместного авторства, менеджеры библиографии и инструменты визуализации данных). С другой, возможна и обратная ситуация - один инструмент может обслуживать несколько этапов (так, некоторые менеджеры библиографии имеют встроенные модули для хранения статей и работы с текстом).

По форме продукта большинство инструментов предназначены для работы в первую очередь с данными и контентом (их поиска, хранения, организации и нахождения). Однако часть инструментов ориентирована на работу со знаниями, среди них:

- сервисы аннотирования статей; 
- сервисы послепубликационной оценки статей;

- исследовательские профили;

- регистрации протоколов исследования;

- онтологии и вики-подобные системы.

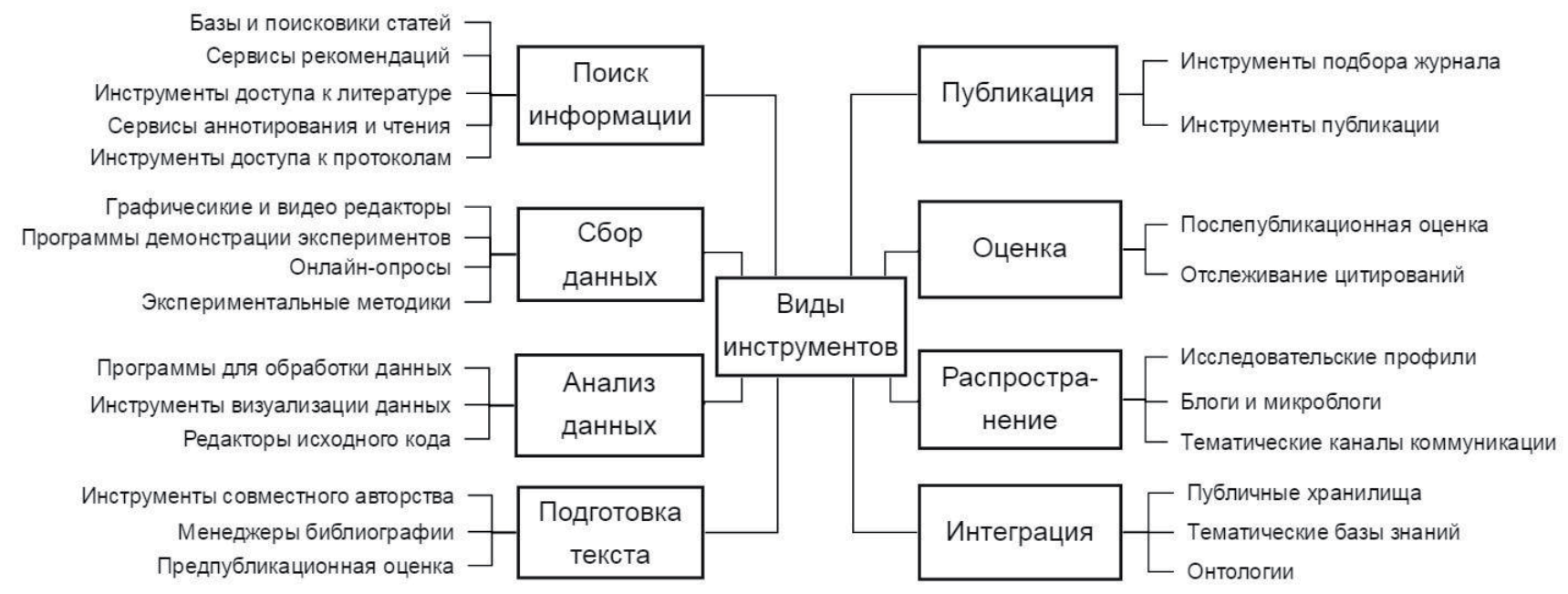

Рисунок 5 - Вариант классификации инструментов работы с продуктом исследования по этапам

Большинство инструментов обеспечивают доступность продукта исследования: либо предусматривают размещение открытой информации, либо обеспечивают эту возможность опционно. Что касается непосредственно распространения информации, инструменты можно разделить на локализованные (предназначенные для работы отдельной научной группы или организации) и интегрирующие (предназначенные для распространения и интеграции информации). Зачастую один и тот же инструмент может обеспечивать и первый, и второй вариант работы. Инструменты, ориентированные на интеграцию, обычно располагают встроенными формами для метаданных, возможностью добавления тегов и системами категорий.

Пример. В процессе подготовки текста используются инструменты для организации списка источников (менеджеры библиографий), для предпубликационной оценки (для редактуры текста), совместного авторства (для подготовки текста), визуализаџии (для графиков и таблии). При этом часть инструментов используется на других этапах: менеджеры библиографий - на этапе поиска публикаций, инструменты визуализации - на этапе обработки данных. Эти инструменты предназначены для работы с информачией в разных формах например, менеджер библиографии в первую очередь предназначен для сбора контента (текстов статей), но многие представители этого класса имеют модули для работы со знаниями (идеями исследователей), например, встроенную возможность добавления заметок к статьям. В частности, менеджеры библиографии могут открывать подготовленные коллекции статей, или обсуждать (в форме комментариев) статьи с другими исследователями.

\section{5 Модель продуктов научного исследования}

Исследование понимается как процесс, состоящий из последовательных итеративных этапов, каждый из которых связан с определённой деятельностью. Результаты исследования представляются в виде конкретных продуктов, которые могут быть соотнесены с этапами, на которых они произведены, и существуют в разных формах. Каждый из продуктов характеризуется доступностью, которая обеспечивается рядом инструментов. В общем виде модель научного исследования представлена на рисунке 6, которая может быть использована следующим образом.

- Для исследовательских коллективов модель может служить основанием для построения своей системы организации получаемых результатов. Зная какие, в каких формах и на ка- 
ком этапе будут получены результаты, исследователи могут подобрать инструментарий, предлагающий как необходимое функциональное обеспечение, так и приемлемый уровень доступности.

- Модель может помочь разработчикам определить место разрабатываемого инструмента в общей структуре инструментального обеспечения и включить в него модули для работы с соответствующими продуктами.

- В зависимости от направленности создаваемых базы или портала знаний модель может быть дополнена онтологиями продуктов исследования и инструментарием специальных ПрО.

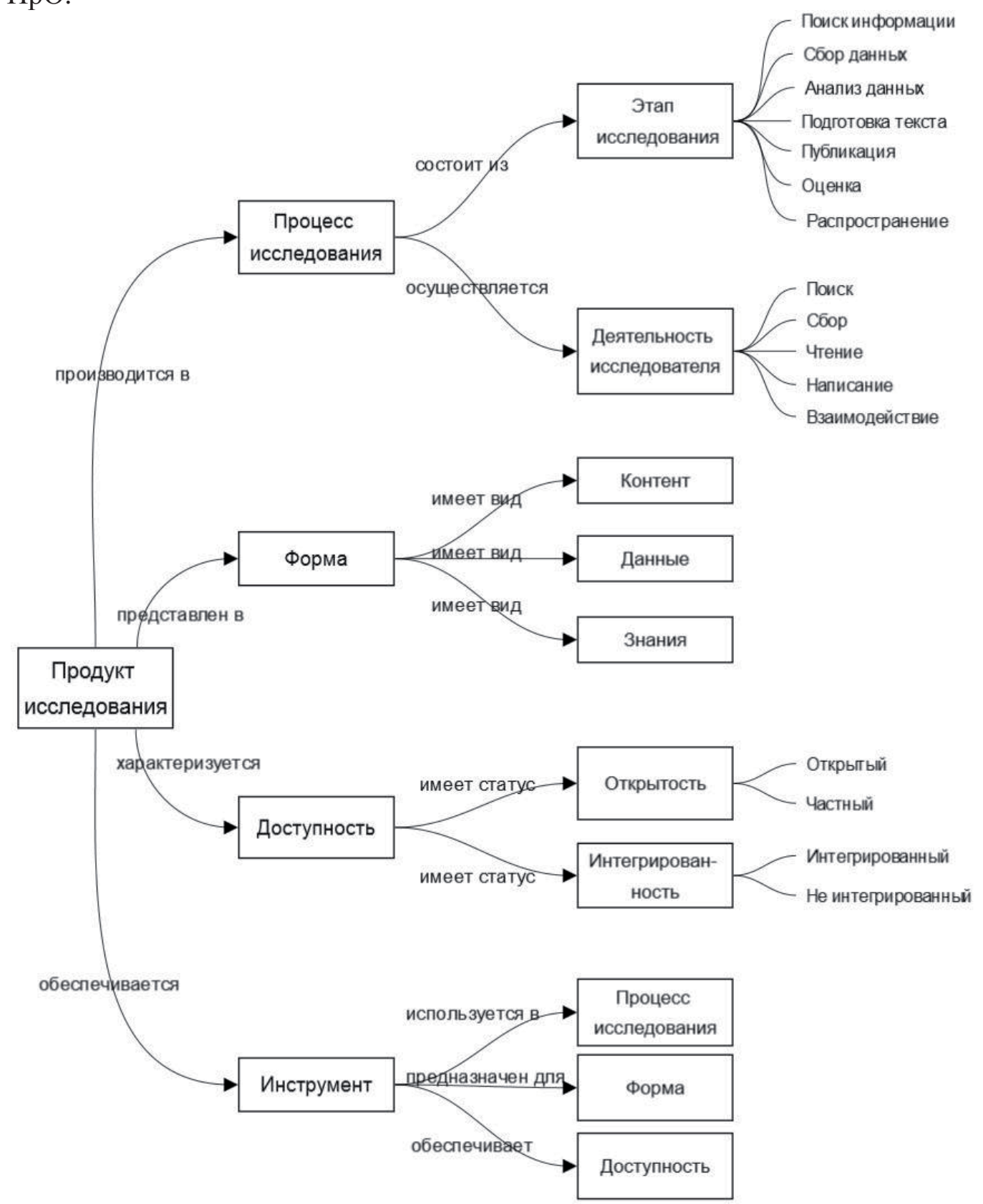

Рисунок 6 - Информационная модель продуктов научного исследования 


\section{Заключение}

Достоинства предложенной модели состоят в том, что она объединяет ряд понятий, характеризующих продукт научного исследования, которые ранее не рассматривались в единой системе и представляет их в форме, которая может быть дополнена и расширена как предметными характеристиками, так и за счёт уточнения общих характеристик продукта исследования.

К недостаткам модели можно отнести слабую детализацию форм продуктов исследования, которые могут быть преодолены расширением модели и её конкретизацией. В статье не были также рассмотрены организационные аспекты исследовательских работ (финансирование, подготовка плана работ и т.д.). Они связаны с продуктами научного исследования и обладают собственным инструментарием. В данной области имеется ряд разработок (например, онтология проекта VIVO [23]), с которыми предложенная информационная модель продуктов научного исследования может быть объединена.

\section{Благодарности}

Автор выражает благодарность Татьяне Альбертовне Гавриловой за ценные комментарии и идеи по усовершенствованию статьи.

\section{Список источников}

[1] Bornmann, L. Growth Rates of Modern Science: A Bibliometric Analysis Based on the Number of Publications and Cited References / L. Bornmann, R. Mutz // J. Assoc. Inf. Sci. Technol. Technol. 2015. Vol. 66, № 11. P. 2215-2222.

[2] Vines, T.H. The availability of research data declines rapidly with article age /T.H. Vines, A.Y.K. Albert, R.L. Andrew, F. Débarre, D.G. Bock, et. al. // Curr. Biol. 2014. Vol. 24, № 1. P. 94-97

[3] Загорулько, Ю.А. Подход к построению порталов научных знаний / Ю.А. Загорулько, О.И. Боровикова// Автометрия. 2008. Т. 44, № 1. С. 100-110.

[4] Загорулько, Г.Б. Разработка онтологии для Интернет-ресурса поддержки принятия решений в слабоформализованных областях / Г.Б. Загорулько // Онтология проектирования. - 2016. - Т. 6, №4(22). - С. 485-500. DOI: 10.18287/2223-9537-2016-6-4-485-500.

[5] Kramer, B. Innovations in scholarly communication - global survey on research tool usage [version 1; referees: 2 approved] / B. Kramer, J. Bosman // F1000Research. 2016. Vol. 5:692. P. 11.

[6] Kubilius, J. A framework for streamlining research workflow in neuroscience and psychology. / J. Kubilius // Front. Neuroinform. 2014. Vol. 7, № January. P. 52.

[7] Nicholas, D. Social media use in the research workflow / D. Nicholas, I. Rowlands // Inf. Serv. Use. 2011. Vol. 31, № November. P. 61-83.

[8] Unsworth, $\boldsymbol{J}$. Scholarly Primitives: what methods do humanities researchers have in common, and how might our tools reflect this? / J. Unsworth // Symposium on Humanities Computing: Formal Methods, Experimental Practice. King's College, London. 2000. Vol. 13. P. 0-5.

[9] Palmer, C.L. Scholarly Information Practices in the Online Environment: Themes from the literature and implications for library service development / C.L. Palmer, L.C. Teffeau, C.M. Pirmann // Scholarly Information Practices in the Online Environment. 2009. 57 p.

[10] Priem, J. Scientometrics 2.0: New metrics of scholarly impact on the social Web / J. Priem, H.A. Piwowar, B.M. Hemminger // First Monday. 2010. Vol. 15, № 7. P. 1-14.

[11] Priem, J. Altmetrics in the wild: Using social media to explore scholarly impact / J. Priem, B.M. Hemminger // arXiv Prepr. arXiv1203.4745. 2012. P. 1-23.

[12] Piwowar, H.A. Value all research products / H.A. Piwowar // Nature. 2013. Vol. 493, № 7431. P. 159.

[13] de Waard, A. Research data management at Elsevier: Supporting networks of data and workflows /A. de Waard // Inf. Serv. Use. 2016. Vol. 36, № 1-2. P. 49-55.

[14] Гаврилова, T.A. Инженерия знаний. Модели и методы. / Т.А. Гаврилова, Д.В. Кудрявцев, Д.И. Муромцев // Учебник. СПб: Лань, 2016. 324 с. 
[15] Bollen, J. A principal component analysis of 39 scientific impact measures / J. Bollen, H. Van de Sompel, A. Hagberg, R. Chute // PLoS One. 2009. Vol. 4, № 6. P. 1-11.

[16] Laakso, M. The development of open access journal publishing from 1993 to 2009 / M. Laakso, P. Welling, H. Bukvova, L. Nyman, B.-C. Björk, T. Hedlund // PLoS One. Public Library of Science, 2011. Vol. 6, № 6. P. e20961.

[17] Björk, B.-C. Open access to the scientific journal literature: situation 2009 / B.-C. Björk, P. Welling, M. Laakso, P. Majlender, T. Hedlund, G. Guðnason // PLoS One. Public Library of Science, 2010. Vol. 5, № 6. P. e11273.

[18] Khabsa, M. The number of scholarly documents on the public web /M. Khabsa, C.L. Giles// PLoS One. 2014. Vol. 9, № 5 .

[19] Kling, $\boldsymbol{R}$. Not just a matter of time: field differences and the shaping of electronic media in supporting scientific communication / R. Kling, G McKim // J. Am. Soc. Inf. Sci. Technol. 2000. Vol. 51, № 14. P. 1306-1320.

[20] Walsh, J.P. Connecting minds: computer-mediated communication and scientific work / J.P. Walsh, S. Kucker, N.G. Maloney, S. Gabbay // J. Am. Soc. Inf. Sci. Technol. 2000. Vol. 51, № 14. P. 1295-1305.

[21] Pearce, N. Digital scholarship considered: how new technologies could transform academic work / N. Pearce, M. Weller, E. Scanlon, S. Kinsley // Educ. 2010. Vol. 16, № 1. P. 33-44.

[22] Pearce, N. A Study of Technology Adoption By Researchers / N. Pearce // Information, Commun. Soc. 2010. Vol. 13, № 8. P. 1191-1206.

[23] Krafft, D.B. VIVO: Enabling National Networking of Scientists / D.B. Kraft, N.A. Cappadona, B. Caruso, J. Corson-Rikert, M. Devare, B.J. Lowe, // Proceedings of the WebSci10: Extending the Frontiers of Society OnLine. 2010.

\title{
INFORMATION MODEL OF SCHOLARLY PRODUCTS
}

\author{
A.M. Begler \\ Saint Petersburg State University, Saint Petersburg, Russia \\ alena.begler@gmail.com
}

\begin{abstract}
Results of the scientific research - scholarly products - are not homogenous. They appear in different forms during different research activities and might be described with several related concepts. First one is the research workflow, which consists of several phases: from searching of the relevant information through data collecting to dissemination of the results. Every phase characterized by the activities, which described by scholarly primitives and the research products are the results of this activities. Second, the research products itself are represented by information of different types: content, data and knowledge. For example, during searching of the relevant information scientist produce several types of information: collection of papers/datasets/etc, several facts, derived from them, and research hypothesis, which express researcher's personal ideas. For producing them modern scientists use research tools (the third concept), which could be distinguished by the information type too, but not always one-by-one: for example, most of the reference management programs are not only storing the papers, but also maintaining the work on the text reading. One more related concept - information availability, reflects openness and discoverability of the scholarly products and might be to the different degree supported by the instruments. We put these four concepts into the information model of the scholarly products, which will be helpful for managing research results.
\end{abstract}

Key words: scholarly information, scholarly products, research lifecycle, research workflow, research tools, research management.

Citation: Begler AM. Information model of the scholarly products. Ontology of designing. 2017; 7(2): 160-171. DOI: 10.18287/2223-9537-2017-7-2-160-171.

\section{References}

[1] Bornmann L., Mutz R. Growth Rates of Modern Science: A Bibliometric Analysis Based on the Number of Publications and Cited References. J. Assoc. Inf. Sci. Technol. Technol. 2015. Vol. 66, № 11. P. 2215-2222. 
[2] Vines TH. The availability of research data declines rapidly with article age. Curr. Biol. 2014. Vol. 24, 1. P. 94-97.

[3] Zagorulko YuA., Borovikova OI. An approach to constructing knowledge portals. Optoelectronics, Instrumentation and Data Processing. Vol. 44. № 1. 2008. P. 75-82.

[4] Zagorulko GB. Development of ontology for intelligent scientific internet resource decision-making support in weakly formalized domains. Ontology of designing. 2016; 6(4): 485-500. DOI: 10.18287/2223-9537-2016-6-4485-500.

[5] Kramer B, Bosman J. Innovations in scholarly communication - global survey on research tool usage [version 1; referees: 2 approved]. F1000Research. 2016. Vol. 5:692. P. 11.

[6] Kubilius $\boldsymbol{J}$. A framework for streamlining research workflow in neuroscience and psychology. Front. Neuroinform. 2014. Vol. 7, № January. P. 52.

[7] Nicholas D, Rowlands I. Social media use in the research workflow. Inf. Serv. Use. 2011. Vol. 31, № November. P. 61-83.

[8] Unsworth J. Scholarly Primitives: what methods do humanities researchers have in common, and how might our tools reflect this? Symposium on Humanities Computing: Formal Methods, Experimental Practice. King's College, London. 2000. Vol. 13. P. 0-5.

[9] Palmer CL, Teffeau LC, Pirmann CM. Scholarly Information Practices in the Online Environment: Themes from the literature and implications for library service development. Scholarly Information Practices in the Online Environment. 2009. 57 p. - http://www.oclc.org/content/dam/research/publications/library/2009/2009-02.pdf.

[10] Priem J., Piwowar HA, Hemminger BM. Scientometrics 2.0: New metrics of scholarly impact on the social Web. First Monday. 2010. Vol. 15, № 7. P. 1-14.

[11] Priem J, Hemminger BM. Altmetrics in the wild: Using social media to explore scholarly impact. arXiv Prepr. arXiv1203.4745. 2012. P. 1-23.

[12] Piwowar HA. Value all research products. Nature. 2013. Vol. 493, № 7431. P. 159.

[13] de Waard A. Research data management at Elsevier: Supporting networks of data and workflows. Inf. Serv. Use. 2016. Vol. 36, № 1-2. P. 49-55.

[14] Gavrilova TA, Kudryavtsev DV, Muromtsev DI. Knowledge engineering. Models and methods [In Russian]. SPb: Lan', 2016. 324 p.

[15] Bollen J, Van de Sompel H, Hagberg A, Chute R. A principal component analysis of 39 scientific impact measures. PLoS One. 2009. Vol. 4, № 6. P. 1-11.

[16] Laakso M, Welling P, Bukvova H, Nyman L, Björk B-C, Hedlund T (2011) The Development of Open Access Journal Publishing from 1993 to 2009. PLoS ONE 6(6): e20961. https://doi.org/10.1371/journal.pone.0020961

[17] Björk B-C, Welling P, Laakso M, Majlender P, Hedlund T, Guðnason G (2010) Open Access to the Scientific Journal Literature: Situation 2009. PLoS ONE 5(6): e11273. https://doi.org/10.1371/journal.pone.0011273

[18] Khabsa M., Giles CL. The number of scholarly documents on the public web. PLoS One. 2014. Vol. 9, № 5.

[19] Kling R, McKim G. Not just a matter of time: field differences and the shaping of electronic media in supporting scientific communication. J. Am. Soc. Inf. Sci. Technol. 2000. Vol. 51, № 14. P. 1306-1320.

[20] Walsh JP, Kucker S, Maloney NG, Gabbay S. Connecting minds: computer-mediated communication and scientific work. J. Am. Soc. Inf. Sci. Technol. 2000. Vol. 51, № 14. P. 1295-1305.

[21] Pearce N, Weller M, Scanlon E, Kinsley S. Digital scholarship considered: how new technologies could transform academic work. Educ. 2010. Vol. 16, № 1. P. 33-44.

[22] Pearce N. A Study of Technology Adoption By Researchers. Information, Commun. Soc. 2010. Vol. 13, № 8. P. 1191-1206.

[23] Krafft D.B., Cappadona NA., Caruso B., Corson-Rikert J., Devare M., Lowe B.J., VIVO: Enabling National Networking of Scientists. Proceedings of the WebSci10: Extending the Frontiers of Society On-Line. 2010.

\section{Сведения об авторах}

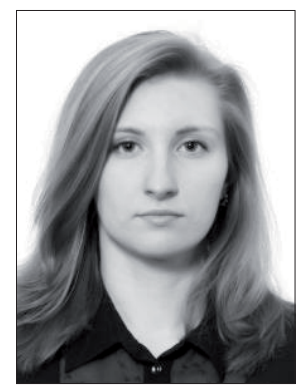

Беглер Алёна Маратовна, 1988 г. рождения. Окончила Санкт-Петербургский государственный университет в 2011 г. С 2014 года - менеджер научной группы, работающей в области когнитивной психологии (факультет психологии СПбГУ). Область научных интересов: организация исследовательского процесса, в особенности - получаемой информации, проектный менеджмент, инженерия знаний.

Alena M. Begler (b. 1988) graduated from the Saint Petersburg State University in 2011. Since 2014 working as a scientific group manager in cognitive psychology field (SPbU Department of Psychology SPbU). Main interests are: research workflow organizing (especially - management of the information products), project management, knowledge engineering. 


\title{
ТЕХНОЛОГИЯ ОПТИМАЛЬНОГО ПРОЕКТИРОВАНИЯ СЛОЖНОСТРУКТУРИРОВАННЫХ СИСТЕМ С РАСПРЕДЕЛЁННЫМИ ПАРАМЕТРАМИ: ПРОГРАММНЫЕ СТРАТЕГИИ ПРИНЯТИЯ РЕШЕНИЙ
}

\author{
Э.Я. Рапопорт, Ю.Э. Плешивцева \\ Институт проблем управления сложными системами РАН, Самара, Россия \\ edgar.rapoport@mail.ru
}

\begin{abstract}
Аннотация
В статье приводятся постановки и предлагаемая технология решения широкого круга задач совместной оптимизации по комплексным критериям эффективности проектных разработок и режимов функционирования сложноструктурированных бесконечномерных детерминированных и не полностью определённых систем с распределёнными параметрами применительно к их описанию операторными уравнениями достаточно общего вида с соответствующими начальными и краевыми условиями. В качестве базовой рассматривается детерминированная задача оптимального проектирования программных управлений режимами функционирования распределённой системы с полным объёмом информации о характеристиках объекта. Предлагаемый метод её решения последовательно распространяется на задачи оптимизации проектных разработок в условиях априори фиксируемых внешних воздействий на объект; на комплексную проблему совместной оптимизации и далее на типичные ситуации оптимального проектирования распределённой системы в условиях интервальной неопределённости её параметрических характеристик и внешних возмущений. Развиваемый подход использует предварительную процедуру параметризации искомых решений, опирающуюся на известные аналитические условия оптимальности; последующую операцию точной редукции к специальным задачам математического программирования; альтернансные свойства их экстремалей, подобные известным результатам теории нелинейных чебышёвских приближений, и фундаментальные закономерности предметной области. Приводится представляющий самостоятельный интерес пример применения полученных результатов общего характера к задаче оптимального проектирования промышленного объекта технологической теплофизики производственного назначения. Предлагаемая интерпретация проблемы оптимального проектирования систем с распределёнными параметрами в виде комплексной задачи совместного поиска соответствующих компонентов векторных пространственно-временных управляющих воздействий и обобщенная на достаточно широкий круг подобных задач технология разработки на этой основе программных стратегий принятия решений являются новыми.
\end{abstract}

Ключевые слова: система с распределёнными параметрами, оптимальное проектирование, полубесконечная оптимизация, альтернансный метод

Цитирование: Рапопорт, Э.Я. Технология оптимального проектирования сложноструктурированных систем с распределёнными параметрами: программные стратегии принятия решений / Э.Я. Рапопорт, Ю.Э. Плешивцева // Онтология проектирования. - 2017. - Т. 7, №2(24). C. 172-190. - DOI: 10.18287/2223-9537-2017-7-2-172-190.

\section{Введение}

Новые возможности решения актуальной проблемы оптимального проектирования технических систем управления появляются при её трактовке в соответствии с современными требованиями системного подхода в качестве задачи совместного поиска проектных разработок и режимов последующего функционирования объекта, обеспечивающих в совокупно- 
сти достижение экстремальной величины априори выбираемого комплексного техникоэкономического критерия оптимальности. Трудности решения подобной задачи существенно увеличиваются с возрастанием сложности структуры объекта и порядка динамических моделей его описания, приобретая принципиальный характер применительно к бесконечномерным системам с распределёнными параметрами (СРП) [1-8].

В целом ряде наиболее характерных для прикладных задач ситуаций подобная совокупная проблема поиска проектных решений и способов организации динамических режимов функционирования СРП может быть сведена к поиску понимаемых в обобщённом смысле соответствующих оптимальных векторных управляющих воздействий (УВ). Их составляющие, относящиеся к проектным разработкам, интерпретируются в своих основных аспектах в роли пространственно-распределённых «статических» УВ, а компоненты, определяющие поведение объекта в динамических режимах - как традиционные, изменяющиеся во временной или пространственно-временной области управления.

Известные условия оптимальности во многих случаях позволяют заведомо установить для типовых моделей СРП базовую программную структуру подобных УВ в форме их явной зависимости от временного и пространственного аргументов с точностью до числа и конкретных значений компонентов конечномерного вектора некоторой определённым образом упорядочиваемой совместной последовательности проектных и режимных параметрических характеристик СРП. Тем самым производится параметризация искомых УВ и последующая точная (в рамках исследуемых моделей) редукция к существенно более простым по сравнению с исходными специальным конечномерным задачам математического программирования, решение которых может быть выполнено применительно к их типичным и некоторым новым постановкам с помощью разработанного авторами конструктивного вычислительного алгоритма («альтернансного» метода), использующего фундаментальные закономерности соответствующей предметной области (ПрО) [8-10]. Подобный подход позволяет реализовать соответствующую программную стратегию оптимального проектирования СРП для достаточно широкого круга ответственных производственных объектов.

Представляющая большой самостоятельный интерес традиционная задача поиска оптимальных программных временных или пространственно-временных УВ в условиях наличия необходимого объёма априорной информации о свойствах объекта может рассматриваться в качестве частной модификации общей задачи оптимального проектирования с заранее фиксируемыми значениями параметрических характеристик СРП.

Ocобое значение в последнем случае приобретает актуальная проблема синтеза оптимальных регуляторов с обратными связями, автоматически реализующими оптимальные режимы функционирования СРП в условиях воздействия на объект внешних и параметрических возмущений (проблема разработки позиционных стратегий управления). Достаточно эффективная процедура её решения в целом ряде типичных ситуаций может быть построена на основе результатов предварительного решения задачи программного управления.

В настоящей статье приводится систематизированное изложение и обобщение предлагаемых авторами конструктивных методов разработки программных стратегий принятия решений для достаточно широкого круга типовых и новых постановок задач оптимального проектирования СРП.

\section{1 Математические модели объектов оптимального проектирования систем с распределёнными параметрами}

Для широкого класса СРП модель поведения управляемой величины $Q$ в зависимости от времени $t$ и пространственных координат $X \in V, X=\left(X_{i}\right), i=\overline{1, m} ; 1 \leq m \leq 3$, в пределах од- 
носвязной области $\overline{\mathrm{V}}$ с кусочно-гладкой границей $\mathrm{S}$ описывается неоднородным скалярным или векторным операторным уравнением

$$
\mathrm{L}_{1}[\mathrm{Q}(X, \mathrm{t}, \mathrm{g}(X), \mathrm{d})]=\mathrm{f}_{1}\left(X, \mathrm{u}_{\mathrm{V}}(X, \mathrm{t}), \mathrm{w}_{1}(X, \mathrm{t})\right), X \in \mathrm{V}, \mathrm{t}>0,
$$

с граничными

$$
\mathrm{L}_{2}[\mathrm{Q}(X, t, g(X), d)]=\mathrm{f}_{2}\left(\mathrm{u}_{\mathrm{s}}(X, t), \mathrm{w}_{2}(X, t)\right), X \in S, t>0
$$

и начальными условиями

$$
\mathrm{L}_{3}\left[\mathrm{Q}(X, \mathrm{t}, \mathrm{g}(X), \mathrm{d}]=\mathrm{f}_{3}(X), X \in \bar{V}, \mathrm{t}=0 .\right.
$$

Здесь $u_{v}(X, t), X \in V \quad$ и $u_{s}(X, t), X \in S$ - зависящие от $X \quad$ и $t$ (пространственновременные) или только от $t$ (сосредоточенные) соответственно внутренние и граничные режимные управляющие воздействия; $\mathrm{g}(\mathrm{X}), \mathrm{X} \in \mathrm{V}$ - пространственно распределённые (статические) управляющие воздействия, в роли которых рассматриваются искомые проектные решения CPП; $\mathbf{w}=\left(\mathrm{Q}(\mathrm{X}, 0), \mathrm{d}, \mathrm{w}_{1}, \mathrm{w}_{2}\right)$ - вектор неопределённых факторов, к которым могут относиться начальное состояние объекта $Q(X, 0)$, неопределённости параметрических характеристик объекта $d$ и внешние возмущающие воздействия $w_{1}$ и $w_{2} ; L_{1}, L_{2}, L_{3}$ - заданные (в общем случае нелинейные) интегро-дифференциальные операторы; $f_{1}(\cdot), f_{2}(\cdot), f_{3}(\cdot)$ - известные скалярные или векторные функции своих аргументов, удовлетворяющие обычным требованиям по их гладкости, и $\mathrm{V}$ - открытая часть области $\bar{V}$, не содержащая её границу.

Краевая задача (1)-(3) может быть решена относительно управляемой величины $Q(X, t) c$ требуемой точностью известными численными или в частных случаях аналитическими методами.

В типичных условиях ограниченности ресурсов на формирование УВ и интервального характера неопределённых факторов будем далее считать, что допустимые значения УВ стеснены на всём протяжении процесса управления при $\mathrm{t} \in[0, \mathrm{~T}]$ ограничениями

$$
\mathrm{u}_{\mathrm{v}}(X, \mathrm{t}) \in \mathrm{U}_{\mathrm{V}}(X, \mathrm{t}) ; \mathrm{u}_{\mathrm{s}}(X, \mathrm{t}) \in \mathrm{U}_{\mathrm{S}}(X, \mathrm{t}), \mathrm{t} \in[0, \mathrm{~T}], \mathrm{g}(X) \in \mathrm{G}(X)
$$

с известными границами заданных множеств $U_{V}, U_{S}, G$, а вся информация о векторе w исчерпывается условиями его принадлежности заданному множеству W его компонентов

$$
\mathrm{w}\left(\mathrm{Q}(\mathrm{X}, 0), \mathrm{d}, \mathrm{w}_{1}(\mathrm{X}, \mathrm{t}), \mathrm{w}_{2}(\mathrm{X}, \mathrm{t})\right) \in \mathrm{W}(\mathrm{X}, \mathrm{t}), \mathrm{t} \in[0, \mathrm{~T}] .
$$

Каждому фиксированному значению $\widetilde{W} \in \mathrm{W}$ в (5) при любом допустимом согласно (4) конкретном наборе управлений $u, u_{s}$ и $g(X)$ отвечает соответствующее пространственновременное распределение $\mathrm{Q}\left(\mathrm{X}, \mathrm{t}, \mathrm{u}_{\mathrm{V}}, \mathrm{u}_{\mathrm{S}}, \mathrm{g}, \tilde{\mathrm{w}}\right)$ («изолированная» траектория [11]) управляемого состояния СРП. Объединение этих состояний по всем допустимым величинам $\mathrm{W} \in \mathrm{W}$ при одних и тех же УВ образует ансамбль траекторий объекта в рассматриваемых условиях ограниченной неопределённости [11]

$$
\mathrm{Q}\left(\mathrm{X}, \mathrm{t}, \mathrm{u}_{\mathrm{v}}, \mathrm{u}_{\mathrm{s}}, \mathrm{g}, \mathrm{T}, \mathrm{W}\right)=\bigcup\left\{\mathrm{Q}\left(\mathrm{X}, \mathrm{t}, \mathrm{u}_{\mathrm{v}}, \mathrm{u}_{\mathrm{s}}, \mathrm{g}, \mathrm{T}, \mathrm{w}: \mathrm{w} \in \mathrm{W}, \mathrm{t} \in[0, \mathrm{~T}]\right)\right\} .
$$

В рамки описания (1)-(6) укладывается ряд наиболее характерных последовательно усложняемых частных ситуаций, на основе которых может быть сформирована технология принятия решений для различных вариантов постановок общей задачи оптимального проектирования СРП.

В качестве типичной базовой модели обычно используется детерминированная модель СРП без учёта неопределённых факторов в (1)-(3) с априори заданным проектным решением 
объекта и искомыми режимными управлениями $u, u_{s}$, выделяющими соответствующую изолированную траекторию из ансамбля (6) при заведомо фиксируемых величинах $\widetilde{\mathbf{w}} \in \mathrm{W}$ и $\widetilde{g} \in \mathrm{G}[1-3,8]$. Подобная модель предусматривает последующее решение частной задачи оптимального проектирования процессов функционирования СРП при полном объёме необходимой информации о характеристиках объекта.

Детерминированная модель СРП (1)-(3) при $\mathrm{w}=\tilde{\mathrm{w}} \in \mathrm{W} ; \mathrm{u}_{\mathrm{v}}=\tilde{\mathrm{u}}_{\mathrm{v}}, \mathrm{u}_{\mathrm{s}}=\tilde{\mathrm{u}}_{\mathrm{s}}$ может быть использована для поиска оптимальных проектных решений $g(X)$ в условиях заранее фиксируемых режимных УВ $\tilde{u}_{v}, \tilde{u}_{s}$, в частности, применительно к статическим режимам работы СРП при $\partial \mathrm{Q} / \partial \mathrm{t}=0$ в (1)-(3).

$\mathrm{B}$ более общей ситуации, требующей организации совместного поиска проектных и режимных решений, следует использовать модель (1)-(3) при $\mathbf{W}=\widetilde{\mathbf{W}}$ с искомыми величинами $u_{v}, u_{\mathrm{s}}$ и $\mathrm{g}$. Фиксация только составляющих $\widetilde{\mathrm{w}}_{1}$ и $\widetilde{\mathrm{w}}_{2}$ в каждой из указанных частных моделей приводит к описанию объекта в условиях интервальной неопределённости начального состояния и параметрических характеристик объекта и отсутствия неопределённых внешних возмущений при $\mathrm{W}=\left(\mathrm{Q}(\mathrm{X}, 0), \mathrm{d}, \widetilde{\mathrm{w}}_{1}, \widetilde{\mathrm{w}}_{2}\right)$.

Аналогичным образом приходим к модели СРП с фиксированными параметрическими характеристиками $\tilde{\mathrm{d}}$ в условиях воздействия множественных возмущений $\mathrm{w}_{1}(\mathrm{t}), \mathrm{w}_{2}(\mathrm{t})$, полагая в (1)-(3) $\mathrm{w}=\left(\mathrm{Q}(\mathrm{X}, 0), \widetilde{d} ; \mathrm{w}_{1}, \mathrm{w}_{2} \in \mathrm{W}\right)[12,13]$.

В двух последних случаях соответствующим образом «сужается» ансамбль траекторий (6).

\section{2 Детерминированная задача оптимального проектирования программных управлений режимами функционирования СРП}

Рассмотрим базовую частную задачу поиска программных оптимальных управлений $u_{v}^{*}, u_{S}^{*}$ применительно к динамической модели СРП (1)-(3) с полным объёмом априорной информации о проектных решениях $\widetilde{g}$, параметрических характеристиках объекта $\widetilde{d}$ и внешних воздействиях $\widetilde{w}_{1}, \widetilde{w}_{2}$ в условиях

$$
\mathbf{W}=\tilde{\mathrm{w}} \in \mathrm{W} ; \mathrm{g}=\widetilde{\mathrm{g}} \in \mathrm{G} .
$$

Искомые управляющие воздействия в большинстве случаев стесняются лишь известными пределами их возможных изменений

$$
\mathrm{u}_{\text {min }} \leq \mathrm{u}(\mathrm{X}, \mathrm{t}) \leq \mathrm{u}_{\max } ; \mathrm{u}_{\mathrm{Smin}} \leq \mathrm{u}_{\mathrm{s}}(\mathrm{X}, \mathrm{t}) \leq \mathrm{u}_{\mathrm{Smax}}, \mathrm{t} \in[0, \mathrm{~T}],
$$

непосредственно характеризующими допустимые множества $U_{V}(X, t)$ и $U_{S}(X, t)$ в (4).

В соответствии с типичными технически реализуемыми условиями по достижению с допустимой погрешностью заданного состояния СРП в конце процесса управления, для большинства представляющих наибольший интерес прикладных задач необходимо обеспечить за время $T$ приближение $\mathrm{Q}(\mathrm{X}, \mathrm{T})$ к требуемому пространственному распределению управляемой величины $\mathrm{Q}^{* *}(\mathrm{X})$ с оцениваемой в равномерной метрике допустимой точностью $\varepsilon>0$ :

$$
\max _{X \in V}\left|Q(X, T)-Q^{* *}(X)\right| \leq \varepsilon \text {. }
$$


Качество процесса управления оценивается в достаточно общем случае по величине $\mathrm{I}_{1}$ максимума по $r$-мерному векторному параметру $\mathrm{y} \in \mathrm{Y} \subset \mathrm{E}^{\mathrm{r}}$ функционала I, являющегося заданной числовой функцией своих аргументов

$$
\mathrm{I}_{1}=\max _{\mathrm{y} \in \mathrm{Y}} \mathrm{I}\left(\mathrm{Q}(\mathrm{X}, \mathrm{t}), \mathrm{u}_{\mathrm{V}}, \mathrm{u}_{\mathrm{S}}, \widetilde{\mathrm{g}}, \widetilde{\mathrm{w}}, \mathrm{T}, \mathrm{y}\right) \rightarrow \min _{\mathrm{u} \in \mathrm{U}_{\mathrm{V}}, \mathrm{u}_{\mathrm{S}} \in \mathrm{U}_{\mathrm{S}}} .
$$

В схему (10) укладываются типичные задачи оптимизации СРП по критериям быстродействия, энергопотребления, минимизации на $\mathrm{V}$ э $X$ отклонений $Q(X, t)$ от $Q^{* *}(X)$ в различных метриках [8, 9].

Исследуемая проблема сводится к определению стесненных ограничениями (8) УВ $u_{v}^{*}, u_{s}^{*}$, которые переводят объект (1)-(3) в условиях (7) из заданного начального в требуемое конечное состояние согласно (9) при минимальном значении критерия оптимальности (10).

В ряде частных случаев данная задача рассматривается в условиях перевода требования (9) в критерий оптимальности вида (10) при $\mathrm{y}=\mathrm{X}$ :

$$
\mathrm{I}_{1}=\max _{\mathrm{X} \in \mathrm{V}}\left|\mathrm{Q}\left(\mathrm{X}, \mathrm{T}, \mathrm{u}_{\mathrm{V}}, \mathrm{u}_{\mathrm{S}}\right)-\mathrm{Q}^{* * *}(\mathrm{X})\right| \rightarrow \min _{\mathrm{u}_{\mathrm{V}}, \mathrm{u}_{\mathrm{S}}}
$$

без ввода дополнительных ограничений на конечное состояние объекта [8, 9].

\section{1 Параметризация управляющих воздействий}

Структура искомых программных управлений $u_{V}^{*}(X, t), u_{S}^{*}(X, t)$ для широкого круга задач оптимизации СРП может быть установлена с использованием известных аналитических условий оптимальности [1-3, 8-10]. Указанным путём во многих случаях может быть получено параметрическое представление этих воздействий $\mathrm{c}$ точностью до вектора $\Delta^{(\mathrm{N})}=\left(\Delta_{\mathrm{i}}^{(\mathrm{N})}\right), \mathrm{i}=\overline{1, \mathrm{~N}}$, определённым образом упорядоченной последовательности конечного числа $\mathrm{N}$ параметров $\Delta_{1}^{(\mathrm{N})}, \Delta_{2}^{(\mathrm{N})}, \ldots, \Delta_{\mathrm{N}}^{(\mathrm{N})}$, непосредственно характеризующих управляющие воздействия в пространственно-временной области их определения (« $\Delta^{(\mathrm{N})}$ параметризация»).

В целом ряде прикладных задач изначально требуется найти управляющие воздействия в заданном классе $\Delta^{(\mathrm{N})}$-параметризуемых функций согласно исходным требованиям, диктуемым техническими возможностями их реализации.

Если непосредственное осуществление операции $\Delta^{(\mathrm{N})}$-параметризации становится затруднительным, то она может быть реализована путём построения конструктивной процедуры отображений на множество $\Omega_{\mathrm{N}}$ допустимых значений $\Delta^{(\mathrm{N})}$ параметров более общей природы, в роли которых выступает, например, применительно к сосредоточенным управляющим воздействиям, набор финишных значений первых $\mathrm{N}$ переменных бесконечной системы сопряженных уравнений принципа максимума Понтрягина при равных нулю конечных значениях остальных её компонент (« $\psi^{(\mathrm{N})}$-параметризация») [10].

\section{2 Редукция к задаче полубесконечной оптимизации}

Интегрирование в аналитической или численной форме уравнений модели объекта (1)(3), (7) с $\Delta^{(\mathrm{N})}$-параметризованными управлениями $u_{v}\left(\Delta^{(\mathrm{N})}, \mathrm{X}, \mathrm{t}\right), \mathrm{u}_{\mathrm{S}}\left(\Delta^{(\mathrm{N})}, \mathrm{X}, \mathrm{t}\right)$ позволяет получить конечное состояние объекта $\mathrm{Q}(\mathrm{X}, \mathrm{T})$ в (9) и значение критерия оптимальности I в 
(10) в форме явных зависимостей $\mathrm{Q}\left(\mathrm{X}, \Delta^{(\mathrm{N})}\right)$ и I $\left(\mathrm{y}, \Delta^{(\mathrm{N})}\right)$ от своих аргументов. При этом минимально достижимые в классе таких управлений значения $\varepsilon_{\min }^{(\mathrm{N})}$ ошибки $\varepsilon$ равномерного приближения $\mathrm{Q}(\mathrm{X}, \mathrm{T})$ к $\mathrm{Q}^{* *}(\mathrm{X})$ в (9)

$$
\varepsilon_{\text {min }}^{(\mathrm{N})}=\min _{\Delta^{(\mathrm{N})} \in \Omega_{\mathrm{N}}}\left\{\max _{\mathrm{X} \in \mathrm{V}}\left|\mathrm{Q}\left(\mathrm{X}, \Delta^{(\mathrm{N})}\right)-\mathrm{Q}^{* *}(\mathrm{X})\right|\right\}
$$

не возрастают (как правило, монотонно убывают) с ростом $\mathrm{N} \in\{\overline{1, \rho}\}$ [9]

$$
\varepsilon_{\min }^{(1)}>\varepsilon_{\min }^{(2)}>\ldots>\varepsilon_{\min }^{(\rho)}=\varepsilon_{\text {inf }} \geq 0,
$$

характеризуя сужающееся к $Q^{* *}(X)$ семейство целевых множеств для $\varepsilon=\varepsilon_{\min }^{(\mathrm{j})}, \mathrm{j}=\overline{1, \rho}$ в (13). Здесь точная нижняя грань $\varepsilon_{\text {inf }}$ достижимых по условию (9) значений $\varepsilon$ в цепочке неравенств (13) оказывается равной минимаксу $\varepsilon_{\min }^{(\rho)}$, где $\rho=\infty$ при $\varepsilon_{\text {inf }}=0$ и $\rho<\infty$ при $\varepsilon_{\text {inf }}>0$ соответственно для управляемых и неуправляемых относительно $\mathrm{Q}^{* *}(\mathrm{X})$ объектов $[1,8,9]$.

В широком классе задач $\Delta^{(\mathrm{N})}$-параметрической оптимизации оптимальные управляющие воздействия $u_{v}^{*}, u_{S}^{*}$ в соответствии с (13) характеризуются минимально возможной для заданного значения $\varepsilon$ в (9) размерностью $\mathrm{N}=\mathrm{N}_{0}$ вектора $\Delta^{(\mathrm{N})}$ по определению минимакса в (12) [8-10]:

$$
\mathrm{N}_{0}=v \forall \varepsilon: \varepsilon_{\min }^{(v)} \leq \varepsilon<\varepsilon_{\min }^{(v-1)}, v \in\{\overline{1, \rho}\}
$$

Отсюда, в частности, следует, что для всех достижимых значений $\varepsilon>0$, удовлетворяющим неравенствам (14), $\Delta^{(\mathrm{N})}$-параметризация оптимальных управлений оказывается конечномерной.

В результате $\Delta^{(\mathrm{N})}$-параметризации производится точная редукция исходной задачи, согласно (9), (10), (12), (13), к задаче полубесконечной оптимизации (ЗПО) на минимум функции $\mathrm{I}_{1}\left(\Delta^{\left(\mathrm{N}_{0}\right)}\right)$ конечного числа переменных $\Delta_{\mathrm{i}}^{\left(\mathrm{N}_{0}\right)}, \mathrm{i}=\overline{1, \mathrm{~N}_{0}}$, с бесконечным числом ограничений, диктуемых требованием (9) для всех $\mathrm{X} \in \mathrm{V}[8,9]$ :

$$
\begin{aligned}
& \mathrm{I}_{1}\left(\Delta^{\left(\mathrm{N}_{0}\right)}\right)=\max _{\mathrm{y} \in \mathrm{Y}} \mathrm{I}\left(\mathrm{Y}, \Delta^{\left(\mathrm{N}_{0}\right)}\right) \rightarrow \min _{\Delta^{\left(\mathrm{N}_{0}\right)} \in \Omega_{\mathrm{N}_{0}}} ; \\
& \Phi_{1}\left(\Delta^{\left(\mathrm{N}_{0}\right)}\right)=\max _{\mathrm{X} \in \mathrm{V}}\left|\mathrm{Q}\left(\mathrm{X}, \Delta^{\left(\mathrm{N}_{0}\right)}\right)-\mathrm{Q}^{*}(\mathrm{X})\right| \leq \varepsilon ; \varepsilon_{\min }^{\left(\mathrm{N}_{0}\right)} \leq \varepsilon<\varepsilon_{\min }^{\left(\mathrm{N}_{0}-1\right)}, \mathrm{N}_{0} \leq \rho .
\end{aligned}
$$

\section{3 Альтернансный метод в задачах параметрической оптимизации СРП}

Решение целого ряда ЗПО вида (15), (16) может быть найдено конструктивным альтернансным методом, распространяющим на ЗПО результаты теории нелинейных чебышёвских приближений $[14,15]$ в условиях некоторых малостеснительных для многих прикладных задач допущений $[8,9]$. Метод базируется на специальных свойствах вектора $\bar{\Delta}^{\left(\mathrm{N}_{0}\right)}$ оптимальных решений ЗПО (15), (16), установленных при указанных допущениях с использованием упрощённых, по сравнению с известными, альтернансных форм необходимых условий экстремума в задачах недифференцируемой оптимизации и дополнительной информации о конфигурации на $\mathrm{V}$ э $\mathrm{X}$ и $\mathrm{Y}$ э у пространственного распределения $\mathrm{Q}\left(\mathrm{X}, \bar{\Delta}^{\left(\mathrm{N}_{0}\right)}\right)$ и $\mathrm{I}\left(\mathrm{y}, \bar{\Delta}^{\left(\mathrm{N}_{0}\right)}\right)$, диктуемой закономерностями ПрО исследуемой конкретной задачи. 
Согласно этим свойствам, одинаковые значения I $\left(y, \bar{\Delta}^{\left(\mathrm{N}_{0}\right)}\right)$, равные $\mathrm{I}_{1}\left(\bar{\Delta}^{\left(\mathrm{N}_{0}\right)}\right)$ в $(10)$, и максимально допустимые отклонения $\left|\mathrm{Q}\left(\mathrm{X}, \bar{\Delta}^{\left(\mathrm{N}_{0}\right)}\right)-\mathrm{Q}^{* *}(\mathrm{X})\right|$, равные $\varepsilon$, достигаются в некоторых точках, соответственно $y_{v}^{0} \in Y, v=\overline{1, R_{y}}$, и $X_{j}^{0} \in V, j=\overline{1, R_{x}}$, суммарное число которых оказывается равным числу всех неизвестных в ЗПО (15), (16), включая $\bar{\Delta}_{\mathrm{i}}^{\left(\mathrm{N}_{0}\right)}, \mathrm{i}=\overline{1, \mathrm{~N}_{0}}$, величину $I_{1}\left(\bar{\Delta}^{\left(\mathrm{N}_{0}\right)}\right)$ и минимакс $\varepsilon_{\min }^{\left(\mathrm{N}_{0}\right)}$, если $\varepsilon=\varepsilon_{\min }^{\left(\mathrm{N}_{0}\right)}$, в соответствии со следующими соотношениями [9]:

$$
\begin{aligned}
& \mathrm{R}_{\mathrm{y}}+\mathrm{R}_{\mathrm{x}}=\mathrm{N}_{0}+1, \text { если } \varepsilon_{\min }^{\left(\mathrm{N}_{0}\right)}<\varepsilon<\widetilde{\varepsilon} ; \\
& \mathrm{R}_{\mathrm{x}}=\mathrm{N}_{0}+1, \text { если } \varepsilon=\varepsilon_{\min }^{\left(\mathrm{N}_{0}\right)} ; \\
& \mathrm{R}_{\mathrm{y}}=\mathrm{N}_{0}+1 \text {, если } \varepsilon \geq \widetilde{\varepsilon},
\end{aligned}
$$

где

$$
\widetilde{\varepsilon}=\Phi_{1}\left(\widetilde{\Delta}^{\left(\mathrm{N}_{0}\right)}\right) ; \widetilde{\Delta}^{\left(\mathrm{N}_{0}\right)}=\arg \inf _{\Delta^{\left(\mathrm{N}_{0}\right)} \in \mathrm{G}_{\mathrm{N}_{0}}} \mathrm{I}_{1}\left(\Delta^{\left(\mathrm{N}_{0}\right)}\right) .
$$

Утверждение (17), дополненное условиями существования экстремума функций $\mathrm{I}\left(\mathrm{y}, \bar{\Delta}^{\left(\mathrm{N}_{0}\right)}\right) \quad$ и $\quad \mathrm{Q}\left(\mathrm{X}, \bar{\Delta}^{(\mathrm{N})}\right)-\mathrm{Q}^{* *}(\mathrm{X}) \quad$ соответственно в $\quad$ точках $\quad \mathrm{y}_{\mathrm{v}_{\mathrm{q}}}^{0} \in \operatorname{int} Y, q=\overline{1, \mathrm{R}_{\mathrm{y} 1}}$, и $X_{j_{p}}^{0} \in \operatorname{int} V, p=\overline{1, R_{X 1}}$, приводит при заданной величине $\varepsilon$ в (16) к определяющей системе равенств

$$
\begin{aligned}
& \mathrm{I}\left(\mathrm{y}_{\mathrm{v}}^{0}, \bar{\Delta}^{\left(\mathrm{N}_{0}\right)}\right)=\mathrm{I}_{1}\left(\bar{\Delta}^{\left(\mathrm{N}_{0}\right)}\right) ; \frac{\partial \mathrm{l}\left(\mathrm{y}_{\mathrm{v}_{\mathrm{q}}}^{0}, \bar{\Delta}^{\left(\mathrm{N}_{0}\right)}\right)}{\partial \mathrm{y}}=0 ; \\
& \mathrm{v}=\overline{1, \mathrm{R}_{\mathrm{y}}} ; \mathrm{q}=\overline{1, \mathrm{R}_{\mathrm{y} 1}} ; \mathrm{R}_{\mathrm{y} 1} \leq \mathrm{R}_{\mathrm{y}} ; \mathrm{y}_{\mathrm{v}_{\mathrm{q}}}^{0} \in\left\{\mathrm{y}_{\mathrm{v}}^{0}\right\} ; \\
& \mathrm{Q}\left(\mathrm{X}{ }_{\mathrm{j}}^{0}, \bar{\Delta}^{\left(\mathrm{N}_{0}\right)}\right)-\mathrm{Q}^{* *}\left(\mathrm{X}_{\mathrm{j}}^{0}\right) \mid=\varepsilon ; \frac{\partial}{\partial \mathrm{X}}\left(\mathrm{Q}\left(\mathrm{X}_{\mathrm{j}_{\mathrm{p}}}^{0}, \bar{\Delta}^{\left(\mathrm{N}_{0}\right)}\right)-\mathrm{Q}^{* *}\left(\mathrm{X}_{\mathrm{j}_{\mathrm{p}}}^{0}\right)\right)=0 ; \\
& \mathrm{j}=\overline{1, \mathrm{R}_{\mathrm{X}}} ; \mathrm{P}=\overline{1, \mathrm{R}_{\mathrm{X} 1}} ; \mathrm{R}_{\mathrm{X} 1} \leq \mathrm{R}_{\mathrm{X}} ; \mathrm{X}_{\mathrm{j}_{\mathrm{p}}}^{0} \in\left\{\mathrm{X}_{\mathrm{j}}^{0}\right\},
\end{aligned}
$$

замкнутой относительно всех искомых параметров.

При наличии дополнительной содержательной информации о конфигурации распределения I $\left(\mathrm{y}, \bar{\Delta}^{\left(\mathrm{N}_{0}\right)}\right)$ на $\mathrm{Y}$ э у и $\mathrm{Q}\left(\mathrm{X}, \bar{\Delta}^{\left(\mathrm{N}_{0}\right)}\right)-\mathrm{Q}^{* *}(\mathrm{X})$ на $\mathrm{V}$ э $\mathrm{X}$ в зависимости от величины $\varepsilon$, позволяющей идентифицировать точки $X{ }_{j}^{0}$ и $y_{v}^{0}$, данные равенства редуцируются к системе $\mathrm{H}+\mathrm{H}_{1}$ уравнений с $\mathrm{H}+\mathrm{H}_{1}$ неизвестными, где $\mathrm{H}=\mathrm{R}_{\mathrm{x}}+\mathrm{R}_{\mathrm{y}}=\mathrm{N}_{0}+1, \mathrm{H}_{1}=r \mathrm{R}_{\mathrm{y} 1}+m \mathrm{R}_{\mathrm{x} 1}$, а в роли неизвестных фигурируют $\mathrm{N}_{0}$ составляющих $\bar{\Delta}_{\mathrm{i}}^{\left(\mathrm{N}_{0}\right)}, \mathrm{i}=\overline{1, \mathrm{~N}_{0}}$, вектора $\bar{\Delta}^{\left(\mathrm{N}_{0}\right)}$; минимакс $\mathrm{I}_{1}\left(\bar{\Delta}^{\left(\mathrm{N}_{0}\right)}\right) ; r \mathrm{R}_{\mathrm{y} 1}$ координат $\mathrm{R}_{\mathrm{y} 1}$ точек $\mathrm{y}_{\mathrm{v}_{\mathrm{q}}}^{0}$ экстремума $\mathrm{I}\left(\mathrm{y}, \bar{\Delta}^{\left(\mathrm{N}_{0}\right)}\right)$ на $\mathrm{Y}$ и $m \mathrm{R}_{\mathrm{X} 1}$ координат $\mathrm{R}_{\mathrm{X} 1}$ точек $X_{j_{p}}^{0}$ экстремума разности $Q\left(X, \bar{\Delta}^{\left(N_{0}\right)}\right)-Q^{* *}(X)$ на $V$.

Подобным образом случай $\varepsilon=\varepsilon_{\min }^{\left(\mathrm{N}_{0}\right)}$ в соответствии с утверждением (18) приводит при $\mathrm{R}_{\mathrm{X}}=\mathrm{N}_{0}+1$ к системе $\mathrm{N}_{0}+m \mathrm{R}_{\mathrm{X} 1}+1$ уравнений (22) с $\mathrm{N}_{0}+m \mathrm{R}_{\mathrm{X} 1}+1$ неизвестными $\bar{\Delta}_{\mathrm{i}}^{\left(\mathrm{N}_{0}\right)}, \mathrm{X}_{\mathrm{j}_{\mathrm{p}}}^{0}$ 
и $\varepsilon_{\min }^{\left(\mathrm{N}_{0}\right)}$, а при $\varepsilon \geq \widetilde{\varepsilon}$ на основании (19) получаем для $\mathrm{R}_{\mathrm{y}}=\mathrm{N}_{0}+1$ систему $\mathrm{N}_{0}+r \mathrm{R}_{\mathrm{y} 1}+1$ уравнений (21) с $\mathrm{N}_{0}+\mathrm{rR}_{\mathrm{y} 1}+1$ неизвестными $\bar{\Delta}_{\mathrm{i}}^{\left(\mathrm{N}_{0}\right)}, \mathrm{y}_{\mathrm{v}_{\mathrm{q}}}^{0}$ и $\mathrm{I}_{1}\left(\bar{\Delta}^{\left(\mathrm{N}_{0}\right)}\right)$.

Для модификации задачи (15), (16) с непрерывно дифференцируемой целевой функцией $\mathrm{I}_{1}\left(\Delta^{\left(\mathrm{N}_{0}\right)}\right)=\mathrm{I}\left(\Delta^{\left(\mathrm{N}_{0}\right)}\right)$, образуемой в частном случае, когда I $\left(\mathrm{y}, \Delta^{\left(\mathrm{N}_{0}\right)}\right)$ не зависит от $y$, только число $R_{X}$ точек $X_{j}^{0}, j=\overline{1, R_{X}}$, становится равным числу искомых значений $\bar{\Delta}_{\mathrm{i}}^{\left(\mathrm{N}_{0}\right)}, \mathrm{i}=\overline{1, \mathrm{~N}_{0}}$ и $\varepsilon_{\min }^{\left(\mathrm{N}_{0}\right)}$ в случае $\varepsilon=\varepsilon_{\min }^{\left(\mathrm{N}_{0}\right)}$, а соответствующая система равенств (17)-(19) упрощается следующим образом:

$$
\begin{aligned}
& \mathrm{R}_{\mathrm{X}}=\mathrm{N}_{0}, \text { если } \varepsilon_{\min }^{\left(\mathrm{N}_{0}\right)}<\varepsilon<\varepsilon_{\min }^{\left(\mathrm{N}_{0}-1\right)} ; \\
& \mathrm{R}_{\mathrm{X}}=\mathrm{N}_{0}+1, \text { если } \varepsilon=\varepsilon_{\min }^{\left(\mathrm{N}_{0}\right)} .
\end{aligned}
$$

В данном случае равенство (23) аналогичным путём приводит к системе $\mathrm{N}_{0}+m \mathrm{R}_{\mathrm{X}}$ уравнений (22) с $N_{0}+m R_{X_{1}}$ неизвестными $\bar{\Delta}_{i}^{\left(N_{0}\right)}, X_{j_{p}}^{0}$ для заданного значения $\varepsilon>\varepsilon_{\min }^{\left(\mathrm{N}_{0}\right)}$, a случай (24) совпадает с (18).

В частном случае (11) задача полубесконечной оптимизации (15), (16) сводится к минимаксной задаче нелинейных чебышёвских приближений

$$
\mathrm{I}_{1}\left(\Delta^{\left(\mathrm{N}_{0}\right)}\right)=\max _{\mathrm{X} \in \mathrm{V}}\left|\mathrm{Q}\left(\mathrm{X}, \Delta^{\left(\mathrm{N}_{0}\right)}\right)-\mathrm{Q}^{* *}(\mathrm{X})\right| \rightarrow \min _{\Delta^{\left(\mathrm{N}_{0}\right)} \in \Omega_{\mathrm{N}_{0}}}
$$

без дополнительных ограничений с последующей редукцией, подобно предыдущим вариантам, равенств (22) в условиях (24) при $\mathrm{I}_{1}\left(\bar{\Delta}^{\left(\mathrm{N}_{0}\right)}\right)=\varepsilon_{\min }^{\left(\mathrm{N}_{0}\right)}$ к расчётной системе уравнений.

Решения относительно указанных неизвестных систем уравнений, конструируемых по специально разработанной вычислительной процедуре в зависимости от величины $\varepsilon$ в (16) на основании равенств (21), (22) [8], содержат искомые решения ЗПО (15), (16) и могут быть найдены стандартными численными методами.

Специфическая проблема редукции соотношений (21), (22) к расчётным системам уравнений во многих случаях может быть решена с привлечением закономерностей ПрО исследуемой задачи управления СРП, позволяющих априори выявить характер поведения на множествах $\mathrm{Y}$ э у и $\mathrm{V}$ э $\mathrm{X}$ функций I $\left(\mathrm{y}, \bar{\Delta}^{\left(\mathrm{N}_{0}\right)}\right)$ и $\mathrm{Q}\left(\mathrm{X}, \bar{\Delta}^{\left(\mathrm{N}_{0}\right)}\right)-\mathrm{Q}^{* *}(\mathrm{X})$ для заданных значений $\varepsilon$ и идентифицировать точки $X_{j}^{0}, y_{v}^{0}$ в (21), (22). В частности, в [9] установлены все возможные варианты формы этих распределений применительно к характерным условиям $\mathrm{Q}^{* *}(\mathrm{X})=\mathrm{const}, \mathrm{Q}(\mathrm{X}, 0)=\mathrm{const}$ при $\varepsilon=\left[\varepsilon_{\mathrm{min}}^{(1)}, \varepsilon_{\mathrm{inf}}\right]$ в базовых задачах оптимального по быстродействию и расходу энергии управления нестационарными температурными полями, описываемыми уравнениями теплопроводности различной пространственной размерности вида (1)-(3) в областях $\mathrm{V}$ канонической формы с типичными внутренними и граничными управляющими воздействиями.

Процедура параметризации УВ, редукция исходной задачи оптимизации к ЗПО и технология её решения альтернансным методом существенно усложняются при использовании векторных УВ (в частности при совокупном применении внутренних и граничных управлений) за счёт возникновения специфической проблемы выстраивания в однозначно фиксируемом порядке конкретной последовательности параметров $\Delta_{\mathrm{i}}^{(\mathrm{N})}, \mathrm{i}=\overline{1, \mathrm{~N}}$ с присвоением каждому из них заранее определяемого номера $i \in\{\overline{1, N}\}$, не меняющегося для всего ряда натуральных значений $N=\overline{1, \rho}$. В зависимости от каждого из формально возможных вариантов 
такой последовательности изменяются величины минимакса в (11), члены цепочки неравенств (13) и размерность $\mathrm{N}_{0}$ искомого решения $\bar{\Delta}^{\left(\mathrm{N}_{0}\right)}$ ЗПО. В типичных для приложений задачах со скалярными внутренними или граничными УВ подобная проблема достаточно просто решается с использованием закономерностей ПрО $[8,9]$. Описываемая схема алгоритмически точного решения краевых задач оптимального управления линейными и нелинейными моделями СРП параболического типа альтернансным методом апробирована при поиске $\Delta^{(\mathrm{N})}-$ и $\psi^{(\mathrm{N})}$-параметризованных сосредоточенных $\quad$ и пространственнораспределённых УВ в задачах оптимизации по ряду базовых технико-экономических критериев применительно к различным процессам технологической теплофизики, в том числе, с учётом фазовых ограничений на температурные и термонапряжённые состояния в процессе управления и других особенностей промышленных технологий $[8,9,16]$.

Предлагаемая технология предварительной параметризации искомых УВ, конечных состояний объекта и критерия оптимальности, последующей процедуры точной редукции к ЗПО, разрешаемой с использованием альтернансных свойств искомых экстремалей и дополнительной информации о закономерностях ПрО, распространяется далее на значительно более широкий круг задач оптимального проектирования детерминированных и не полностью определённых моделей СРП.

Ниже рассматриваются наиболее характерные постановки таких задач, сводимые к ЗПО различного вида, решения которых укладываются в общую схему (15)-(16) альтернансного метода.

\section{3 Детерминированная задача оптимизации проектных решений в условиях заданных режимных управлений}

Данная частная задача оптимального проектирования СРП сводится к поиску статического распределённого управления $\mathrm{g}^{*}(X) \in \mathrm{G}$, которое переводит объект (1)-(5) в условиях фиксированных воздействий $\mathrm{w}=\widetilde{\mathrm{w}} \in \mathrm{W}$ и режимных управлений $\mathrm{u}_{v}=\tilde{u}_{v}, \mathrm{u}_{\mathrm{s}}=\tilde{\mathrm{u}}_{\mathrm{s}}$ в требуемое конечное состояние согласно (9) с минимальным значением критерия оптимальности

$$
\mathrm{I}_{2}=\max _{\mathrm{y} \in \mathrm{Y}} \mathrm{I}\left(\mathrm{Q}(X, \mathrm{t}), \tilde{\mathrm{u}}_{\mathrm{v}}, \tilde{\mathrm{u}}_{\mathrm{s}}, \mathrm{g}(\mathrm{X}), \tilde{\mathrm{w}}, \mathrm{T}, \mathrm{y}\right) \rightarrow \min _{\mathrm{g} \in \mathrm{G}}
$$

вместо (10).

Искомое проектное решение $g^{*}(X)$ может быть найдено по описанной выше общей схеме определения режимных управлений $u_{v}^{*}$ и $u_{s}^{*}$ путём редукции исходной задачи к ЗПО вида (15), (16), разрешаемой теперь уже относительно вектора $\Delta_{\mathrm{g}}^{\left(\mathrm{N}_{\mathrm{g}}\right)}=\left(\Delta_{\mathrm{i}}^{\left(\mathrm{N}_{\mathrm{g}}\right)}\right), \mathrm{i}=\overline{1, \mathrm{~N}_{\mathrm{g}}}$, параметрического представления $\mathrm{g}^{*}(\mathrm{X})$

$$
\begin{aligned}
& \mathrm{I}_{2}\left(\Delta_{\mathrm{g}}^{\left(\mathrm{N}_{\mathrm{g} 0}\right)}\right)=\max _{\mathrm{y} \in \mathrm{Y}} \mathrm{I}\left(\mathrm{y}, \Delta_{\mathrm{g}}^{\left(\mathrm{N}_{\mathrm{g} 0}\right)}\right) \rightarrow \min _{\Delta_{\mathrm{g}}^{\left(\mathrm{N}_{\mathrm{g} 0}\right)} \in \Omega_{\mathrm{g} 0}} ; \\
& \Phi_{2}\left(\Delta_{\mathrm{g}}^{\left(\mathrm{N}_{\mathrm{g} 0}\right)}\right)=\max _{\mathrm{X} \in \mathrm{V}}\left|\mathrm{Q}\left(\mathrm{X}, \Delta_{\mathrm{g}}^{\left(\mathrm{N}_{\mathrm{g} 0}\right)}\right)-\mathrm{Q}^{* *}(\mathrm{X})\right| \leq \varepsilon ; \varepsilon_{\min }^{\left(\mathrm{N}_{\mathrm{g} 0}\right)} \leq \varepsilon<\varepsilon_{\min }^{\left(\mathrm{N}_{\mathrm{g} 0}-1\right)} .
\end{aligned}
$$

Представляющая самостоятельный интерес задача оптимизации проектных решений объекта на стадии его функционирования в стационарном состоянии $\mathrm{Q}_{\mathrm{c}}(\mathrm{X})$ при

$$
\lim _{\mathrm{t} \rightarrow \infty} \partial \mathrm{Q} / \partial \mathrm{t}=0 ; \lim _{\mathrm{t} \rightarrow \infty} \mathrm{u}_{\mathrm{v}}=\text { const; } \lim _{\mathrm{t} \rightarrow \infty} \mathrm{u}_{\mathrm{s}}=\text { const }
$$

во многих случаях сводится к виду (11) 
(29)

$$
\mathrm{I}_{2}=\max _{X \in \mathrm{V}}\left|\mathrm{Q}_{\mathrm{c}}(\mathrm{X}, \mathrm{g}(\mathrm{X}))-\mathrm{Q}^{* *}(\mathrm{X})\right| \rightarrow \min _{\mathrm{g}(\mathrm{X}) \in \mathrm{G}} .
$$

Процедура $\Delta_{\mathrm{g}}^{\left(\mathrm{N}_{\mathrm{g}}\right)}$-параметризации $\mathrm{g}(\mathrm{X})$, аналогичная $\Delta^{(\mathrm{N})}$-параметризации режимных управлений, приводит к параметрическому представлению $g\left(X, \Delta_{g}^{\left(N_{g}\right)}\right), Q_{c}\left(X, \Delta_{g}^{\left(N_{g}\right)}\right)$ и $\mathrm{I}_{2}\left(\Delta_{\mathrm{g}}^{\left(\mathrm{N}_{\mathrm{g}}\right)}\right)$ управляющего воздействия, стационарного состояния СРП и критерия оптимальности.

Последующая редукция к нелинейной задаче чебышёвских приближений вида (25)

$$
\mathrm{I}_{2}\left(\Delta_{\mathrm{g}}^{\left(\mathrm{N}_{\mathrm{g} 0}\right)}\right)=\max _{\mathrm{X} \in \mathrm{V}}\left|\mathrm{Q}_{\mathrm{C}}\left(\mathrm{X}, \Delta_{\mathrm{g}}^{\left(\mathrm{N}_{\mathrm{g} 0}\right)}\right)-\mathrm{Q}^{* *}(\mathrm{X})\right| \rightarrow \min _{\Delta_{\mathrm{g}}^{\left(\mathrm{N}_{\mathrm{g} 0}\right)} \in \Omega_{\mathrm{N}_{\mathrm{g} 0}}}
$$

позволяет найти ее решение $\bar{\Delta}_{\mathrm{g}}^{\left(\mathrm{N}_{\mathrm{g} 0}\right)}$ с требуемой точностью по схеме альтернансного метода.

Специфической особенностью задачи (30) является необходимость попутного определения границ области G в (4), исходя из фундаментальных законов сохранения энергетического или материального баланса в стационарном режиме работы СРП.

\section{4 Детерминированная задача совместной оптимизации проектных решений и режимов функционирования СРП}

Общая детерминированная задача оптимального проектирования СРП (1)-(5) в отличие от рассмотренных в разделах 2 и 3 заключается в совместном определении допустимых проектных решений $g^{*}(X)$ и режимных управлений $u_{v}^{*}$ и $u_{s}^{*}$, обеспечивающих в условиях известного вектора $\mathbf{W}=\widetilde{\mathbf{W}} \in \mathrm{W}$ достижение требуемых согласно (9) конечных состояний объекта (1)-(5) с минимальным значением критерия оптимальности

$$
\mathrm{I}_{3}=\max _{\mathrm{y} \in \mathrm{Y}} \mathrm{I}\left(\mathrm{Q}(\mathrm{X}, \mathrm{t}), \mathrm{u}_{\mathrm{Y}}, \mathrm{u}_{\mathrm{S}}, \mathrm{g}, \widetilde{\mathrm{w}}, \mathrm{T}, \mathrm{y}\right) \rightarrow \min _{\mathrm{u}_{\mathrm{v}}, \mathrm{u}_{\mathrm{s}}, \mathrm{g}}
$$

вместо $\mathrm{I}_{1}, \mathrm{I}_{2}$ в (10), (26) и (29).

Процедура $\Delta^{(\mathrm{N})}$ и $\Delta_{\mathrm{g}}^{\left(\mathrm{N}_{\mathrm{g}}\right)}$-параметризации искомых воздействий $\mathrm{u}_{\mathrm{v}}, \mathrm{u}_{\mathrm{s}}$ и $\mathrm{g}(\mathrm{X})$ соответственно, формирование расширенного вектора параметров повышенной размерности $\Delta_{\Sigma}^{\left(\mathrm{N}+\mathrm{N}_{\mathrm{g}}\right)}=\left(\Delta^{(\mathrm{N})}, \Delta_{\mathrm{g}}^{\left(\mathrm{N}_{\mathrm{g}}\right)}\right)$ и последующее интегрирование уравнений модели объекта (1)-(5) с параметризованными управлениями $\mathrm{u}_{v}\left(\Delta^{(\mathrm{N})}, \mathrm{X}, \mathrm{t}\right), \mathrm{u}_{\mathrm{s}}\left(\Delta^{(\mathrm{N})}, \mathrm{X}, \mathrm{t}\right), \mathrm{g}\left(\mathrm{X}, \Delta^{\left(\mathrm{N}_{\mathrm{g}}\right)}\right)$ приводят к параметрическим зависимостям $\mathrm{Q}\left(\mathrm{X}, \Delta_{\Sigma}^{\left(\mathrm{N}+\mathrm{N}_{\mathrm{g}}\right)}\right), \mathrm{I}\left(\mathrm{y}, \Delta_{\Sigma}^{\left(\mathrm{N}+\mathrm{N}_{\mathrm{g}}\right)}\right)$ конечного состояния объекта и критерия оптимальности (31) от своих аргументов. В результате опять обеспечивается редукция исходной задачи к ЗПО вида (15), (16):

$$
\begin{aligned}
& \mathrm{I}_{3}\left(\Delta_{\Sigma}^{\left(\mathrm{N}_{0}+\mathrm{N}_{\mathrm{g} 0}\right)}\right)=\max _{\mathrm{y} \in \mathrm{Y}} \mathrm{I}\left(\mathrm{Y}, \Delta_{\Sigma}^{\left(\mathrm{N}_{0}+\mathrm{N}_{\mathrm{g} 0}\right)}\right) \rightarrow \min _{\Delta_{\Sigma}^{\left(\mathrm{N}_{0}+\mathrm{N}_{\mathrm{g} 0}\right)} \in \Omega_{\mathrm{N}_{0}+\mathrm{N}_{\mathrm{g}}}} ; \\
& \Phi_{3}\left(\Delta_{\Sigma}^{\left(\mathrm{N}_{0}+\mathrm{N}_{\mathrm{g} 0}\right)}\right)=\max _{\mathrm{X} \in \mathrm{V}}\left|\mathrm{Q}\left(\mathrm{X}, \Delta_{\Sigma}^{\left(\mathrm{N}_{0}+\mathrm{N}_{\mathrm{g} 0}\right)}\right)-\mathrm{Q}^{* * *}(\mathrm{X})\right| \leq \varepsilon ; \varepsilon_{\min }^{\left(\mathrm{N}_{0}+\mathrm{N}_{\mathrm{g} 0}\right)} \leq \varepsilon<\varepsilon_{\min }^{\left(\mathrm{N}_{0}+\mathrm{N}_{\mathrm{g} 0}-1\right)},
\end{aligned}
$$

(или в частных случаях, подобных (11), к ЗПО вида (25), (30)), снова разрешаемой по общей предлагаемой схеме относительно искомой величины $\bar{\Delta}_{\Sigma}^{\left(\mathrm{N}_{0}+\mathrm{N}_{\mathrm{g} 0}\right)}$. Здесь размерность $\mathrm{N}_{0}+\mathrm{N}_{\mathrm{g} 0}$ вектора $\bar{\Delta}_{\Sigma}^{\left(\mathrm{N}_{0}+\mathrm{N}_{\mathrm{g}}\right)}$ опять определяется по правилу аналогичному (14) применительно к по- 
добной (13) цепочке неравенств для величин минимаксов, определяемых согласно (12) с заменой $\mathrm{N}$ на $\mathrm{N}+\mathrm{N}_{\mathrm{g}}$.

Векторный характер искомых управлений приводит к необходимости решения с помощью закономерностей ПрО, указанной в разделе 1 , вспомогательной задачи формирования упорядоченной последовательности компонентов вектора $\Delta_{\Sigma}^{\left(\mathrm{N}+\mathrm{N}_{\mathrm{g}}\right)}=\left(\Delta_{\Sigma 1}^{\left(\mathrm{N}+\mathrm{N}_{\mathrm{g}}\right)}, \Delta_{\Sigma 2}^{\left(\mathrm{N}+\mathrm{N}_{\mathrm{g}}\right)}, \ldots, \Delta_{\Sigma\left(\mathrm{N}+\mathrm{N}_{\mathrm{g}}\right)}^{\left(\mathrm{N}+\mathrm{N}_{\mathrm{g}}\right)}\right.$, число $\mathrm{N}+\mathrm{N}_{\mathrm{g}}$ которых увеличивается с возрастанием $\mathrm{N}$ и $\mathrm{N}_{g}$.

\section{5 Задача оптимизации проектных решений СРП в условиях интервальной неопределённости параметрических характеристик объекта}

Самостоятельный интерес представляет задача оптимального проектирования СРП (1)(6) в характерных условия интервальной неопределённости вектора $\mathrm{b}=\left(\mathrm{d}, \mathrm{Q}_{0}\right)=\left(\mathrm{d}_{\mathrm{j}}, \mathrm{j}=\overline{1, \mathrm{r}} ; \mathrm{Q}_{0}\right) \in \mathrm{W}_{1} \subset \mathrm{W}$ неизменных во времени параметрических характеристик объекта, к которым во многих случаях можно отнести параметрическое представление начального состояния объекта $\mathrm{Q}(\mathrm{X}, 0)=\mathrm{Q}_{0}=$ const $\forall \mathrm{X} \in \mathrm{V}$, где

$$
W_{1}=\left\{d_{j}, Q_{0}: d_{j \min } \leq d_{j} \leq d_{j \max } ; Q_{0 \text { min }} \leq Q_{0} \leq Q_{0 \text { max }}, j=\overline{1, r}\right\} .
$$

При заданных внешних воздействиях $\widetilde{w}_{1}, \widetilde{w}_{2} \in W$ требования вида (9) должны быть выполнены для всех конечных состояний ансамбля (6)

$$
\mathrm{Q}\left(\mathrm{X}, \mathrm{T}, \mathrm{u}_{\mathrm{v}}, \mathrm{u}_{\mathrm{s}}, \mathrm{g}, \mathrm{W}\right)=\bigcup\left\{\mathrm{Q}\left(\mathrm{X}, \mathrm{T}, \mathrm{u}_{\mathrm{v}}, \mathrm{u}_{\mathrm{s}}, \mathrm{g}, \widetilde{\mathrm{w}}_{1}, \widetilde{\mathrm{w}}_{2}, \mathrm{~b} \in \mathrm{W}_{1} \subset \mathrm{W}\right)\right\},
$$

образуемого всеми допустимыми согласно (33) реализациями значений $b$ :

$$
\max _{b \in \mathbb{W}_{1}}\left[\max _{X \in V}\left|Q(X, T, b)-Q^{* *}(X)\right|\right] \leq \varepsilon
$$

В таком случае задача сводится к совместному определению $u_{v}^{*}, u_{s}^{*}, g^{*}$, обеспечивающих при $\mathrm{w}_{1}=\widetilde{\mathrm{w}}_{1}, \mathrm{w}_{2}=\widetilde{\mathrm{w}}_{2}$ перевод ансамбля траекторий (6) в требуемое конечное состояние (35) c минимальным значением функционала качества $\mathrm{I}_{4}$, определяемого по принципу гарантированного результата в аналогичной (10) форме функции максимума:

$$
\mathrm{I}_{4}=\max _{b \in \mathrm{W}_{1}} \mathrm{I}\left(\mathrm{Q}(\mathrm{X}, \mathrm{t}), \mathrm{u}_{\mathrm{v}}, \mathrm{u}_{\mathrm{s}}, \mathrm{g}, \widetilde{\mathrm{w}}_{1}, \widetilde{\mathrm{w}}_{2}, \mathrm{~b}\right) \rightarrow \min _{\mathrm{u}_{v}, u_{s}, g} .
$$

Подобная (27) $\Delta_{\Sigma}^{\left(\mathrm{N}+\mathrm{N}_{\mathrm{g}}\right)}$-параметризация искомых воздействий приводит к редукции исходной задачи (35), (36) к ЗПО вида (32) c $r+1$-мерным векторным параметром $\mathrm{y}=\mathrm{b}$

$$
\begin{aligned}
& \mathrm{I}_{4}\left(\Delta_{\Sigma}^{\left(\mathrm{N}_{0}+\mathrm{N}_{g_{0}}\right)}\right)=\max _{\mathrm{b} \in \mathrm{W}_{1}} \mid\left(\mathrm{b}, \Delta_{\Sigma}^{\left(\mathrm{N}_{0}+\mathrm{N}_{g_{0}}\right)}\right) \rightarrow \min _{\Delta_{\Sigma}^{\left(\mathrm{N}_{0}+\mathrm{N} g_{0}\right)} \in \Omega_{\mathrm{N}_{0}+\mathrm{N}_{g_{0}}}} ; \\
& \Phi_{4}\left(\Delta_{\Sigma}^{\left(\mathrm{N}_{0}+\mathrm{N}_{\mathrm{g}}\right)}\right)=\max _{\mathrm{Z} \in \mathrm{Z}}\left|\mathrm{Q}\left(\mathrm{Z}, \Delta_{\Sigma}^{\left(\mathrm{N}_{0}+\mathrm{N}_{g_{0}}\right)}\right)-\mathrm{Q}^{* * *}(\mathrm{X})\right| \leq \varepsilon ; \varepsilon_{\min }^{\left(\mathrm{N}_{0}+\mathrm{N}_{\mathrm{g}_{0}}\right)} \leq \varepsilon<\varepsilon_{\min }^{\left(\mathrm{N}_{0}+\mathrm{N}_{g_{0}}-1\right)} ; \\
& \mathrm{Z}=(\mathrm{X}, \mathrm{b}) ; \mathrm{Z}=\mathrm{V} \times \mathrm{W}_{1} \in \mathrm{E}^{\mathrm{m}+\mathrm{r}+1}
\end{aligned}
$$

и континуумом ограничений, рассматриваемых на расширенном по сравнению с (32) множестве $Z$ элементов $Z \in Z$, включающем наряду с пространственными переменными $X \in V$ допустимые значения вектора $b \in W_{1}$. 
Возникающая при этом дополнительная проблема выявления характера распределения $\mathrm{Q}\left(\mathrm{Z}, \bar{\Delta}_{\Sigma}^{\left(\mathrm{N}_{0}+\mathrm{N}_{\mathrm{g} 0}\right)}\right)-\mathrm{Q}^{* *}(\mathrm{X})$ на множестве $\mathrm{Z}$ в целях редукции системы равенств вида (21), (22) к расчётным системам уравнений альтернансного метода во многих случаях может быть решена на основе свойств результирующих состояний СРП для детерминированных состояний объекта и фундаментальных закономерностей ПрО [8-10, 16]. В наиболее характерных ситуациях точки $z_{j}^{0}=\left(x_{j}^{0}, b_{j}^{0}\right), j=\overline{1, R_{x}}$ в которых выполняются подобно (22) равенства $\left|\mathrm{Q}\left(\mathrm{Z}_{\mathrm{j}}^{0}, \bar{\Delta}_{\Sigma}^{\left(\mathrm{N}_{0}+\mathrm{N}_{\mathrm{g} 0}\right)}\right)-\mathrm{Q}^{* *}\left(\mathrm{X}_{\mathrm{j}}^{0}\right)\right|=\varepsilon$, содержат комбинации предельно допустимых значений $\mathrm{d}_{\mathrm{j}}$ на границах множества $\mathrm{W}_{1}$.

\section{6 Программная реализация обратных связей в задачах оптимизации режимов функционирования СРП при наличии внешних возмущений}

В типичных условиях воздействия на реальный объект с заданными проектными решениями $\mathrm{g}=\widetilde{\mathrm{g}} \in \mathrm{G}$ в (4) и заранее фиксируемыми параметрическими характеристиками $\mathrm{d}=\widetilde{\mathrm{d}} \in \mathrm{W}_{1}$, множественных возмущений $\mathrm{w}_{1}, \mathrm{w}_{2} \in \mathrm{W}$ в (5), вся информация о которых исчерпывается заданием их граничных значений в пределах допустимой области $\mathrm{W}$ их изменения, возникает задача поиска оптимальных программных управлений $u_{V}^{*}, u_{S}^{*}$ ансамблем траекторий

$$
\mathrm{Q}\left(\mathrm{X}, \mathrm{t}, \mathrm{u}_{\mathrm{v}}, \mathrm{u}_{\mathrm{s}}, \widetilde{\mathrm{g}}, \mathrm{T}, \widetilde{\mathrm{d}}, \mathrm{W}\right)=\bigcup\left\{\mathrm{Q}\left(\mathrm{X}, \mathrm{t}, \mathrm{u}_{\mathrm{v}}, \mathrm{u}_{\mathrm{s}}, \widetilde{\mathrm{g}}, \mathrm{T}, \widetilde{\mathrm{d}}, \mathrm{Q}_{0}, \mathrm{w}_{1}, \mathrm{w}_{2} \in \mathrm{W}\right)\right\}, \mathrm{t} \in[0, \mathrm{~T}] .
$$

Эта задача может быть записана в подобной (35), (36) форме

$$
\begin{aligned}
& \max _{\mathrm{Q}_{0}, \mathrm{w}_{1}, \mathrm{w}_{2} \in \mathrm{W}}\left[\max _{X \in \mathrm{V}}\left|\mathrm{Q}\left(X, T, \mathrm{Q}_{0}, \mathrm{w}_{1}, \mathrm{w}_{2}\right)-\mathrm{Q}^{* *}(\mathrm{X})\right|\right] \leq \varepsilon ; \\
& \mathrm{I}_{5}=\max _{\mathrm{Q}_{0}, \mathrm{w}_{1}, \mathrm{w}_{2} \in \mathrm{W}} \mathrm{I}\left(\mathrm{Q}(\mathrm{X}, \mathrm{t}), \mathrm{u}_{\mathrm{v}}, \mathrm{u}_{\mathrm{s}}, \widetilde{\mathrm{g}}, \tilde{\mathrm{d}}, \mathrm{Q}_{0}, \mathrm{w}_{1}, \mathrm{w}_{2}\right) \rightarrow \min _{\mathrm{u}_{\mathrm{v}}, \mathrm{u}_{\mathrm{s}}}
\end{aligned}
$$

с последующей редукцией после параметризации искомых управлений к ЗПО вида (37):

$$
\begin{aligned}
& \mathrm{I}_{5}\left(\Delta_{\Sigma}^{\left(\mathrm{N}_{0}\right)}\right)=\max _{\mathrm{Q}_{0}, \mathrm{w}_{1}, \mathrm{w}_{2} \in \mathrm{W}} \mathrm{I}\left(\Delta_{\Sigma}^{\left(\mathrm{N}_{0}\right)}, \mathrm{Q}_{0}, \mathrm{~W}_{1}, \mathrm{~W}_{2}\right) \rightarrow \min _{\Delta_{\Sigma}^{\left(\mathrm{N}_{0}\right)} \in \Omega_{\mathrm{N}_{0}}} \\
& \Phi_{5}\left(\Delta_{\Sigma}^{\left(\mathrm{N}_{0}\right)}\right)=\max _{\mathrm{Z}_{1} \in \mathrm{Z}_{1}}\left|\mathrm{Q}\left(\mathrm{Z}_{1}, \Delta_{\Sigma}^{\left(\mathrm{N}_{0}\right)}\right)-\mathrm{Q}^{* *}(\mathrm{X})\right| \leq \varepsilon ; \varepsilon_{\min }^{\left(\mathrm{N}_{0}\right)} \leq \varepsilon<\varepsilon_{\min }^{\left(\mathrm{N}_{0}-1\right)}, \\
& \mathrm{Z}_{1}=\left(\mathrm{X}, \mathrm{Q}_{0}, \mathrm{~W}_{1}, \mathrm{~W}_{2}\right) ; \mathrm{Z}_{1}=\mathrm{V} \times \mathrm{W}_{2} ; \mathrm{W}_{2}=\left(\mathrm{Q}_{0}, \mathrm{~W}_{1}, \mathrm{~W}_{2}\right) \in \mathrm{W} .
\end{aligned}
$$

Описанная стратегия программного управления по принципу гарантированного результата ансамблем траекторий СРП, порождаемым всеми допустимыми реализациями учитываемых возмущающих воздействий, заведомо приводит к существенным потерям по минимизируемому критерию качества по сравнению с замкнутыми системами автоматического управления, обеспечивающими требуемый уровень подавления возмущений. Однако проблема синтеза соответствующих оптимальных автоматических регуляторов отличается известными затруднениями принципиального характера $[11,13]$.

Возможный путь их преодоления состоит в переходе от трудноразрешимой задачи построения в явной форме алгоритма оптимального управления с обратными связями к поиску реализующей искомый закон регулирования последовательности программных оптимальных управлений $u_{k k}^{*}(X, t), u_{s k}^{*}(X, t), t \in\left[t_{k}, T\right], 0 \leq t_{k}<T$, для каждого из которых начальное на ин- 
тервале $\left[t_{k}, T\right]$ состояние ансамбля $\left.\mathrm{Q}\left(\mathrm{X}, \mathrm{t}, \mathrm{u}, \mathrm{u}_{\mathrm{s}}, \widetilde{\mathrm{g}}, \mathrm{T}, \tilde{\mathrm{d}}, \mathrm{W}\right)\right|_{\mathrm{t}=\mathrm{t}_{\mathrm{k}}}$ в (38) вычисляется в заранее фиксируемые на временной сетке с заданным периодом квантования $\mathrm{h}$ моменты времени $t_{k}=k h, k=\overline{0, \sigma-1}, \sigma h=T$ по сигналам $\mathrm{Q}_{\mathrm{u}}\left(\mathrm{X}, \mathrm{t}_{\mathrm{k}}\right)$ измерения текущего состояния объекта $\mathrm{c}$ помощью известных способов его наблюдения [12, 13].

В таком случае исходная минимаксная задача оптимизации (39), (40), сформулированная на всём временном промежутке [0,T], сводится к последовательно разрешаемым на интервалах $\left[t_{k}, T\right]$ э $t, k=\overline{0, \sigma-1}$, задачам вида (39), (40) программного управления «суживающимися» по мере возрастания $t_{k}$ ансамблями траекторий $\mathrm{Q}\left(\mathrm{X}, \mathrm{t}, \mathrm{u}_{\mathrm{k}}, \mathrm{u}_{\mathrm{sk}}, \widetilde{\mathrm{g}}, \mathrm{T}, \widetilde{\mathrm{d}}, \mathrm{w}_{1 \mathrm{k}}, \mathrm{w}_{2 \mathrm{k}}\right)$; $\mathrm{w}_{1 \mathrm{k}}, \mathrm{w}_{2 \mathrm{k}} \in \mathrm{W}, \mathrm{t} \in\left[\mathrm{t}_{\mathrm{k}}, \mathrm{T}\right]$, с наблюдаемыми начальными состояниями $\mathrm{Q}_{\mathrm{u}}\left(\mathrm{X}, \mathrm{t}_{\mathrm{k}}\right)$. При этом искомые оптимальные управления $u_{v}^{*}, u_{S}^{*}$ на всём протяжении процесса управления компонуются из участков изменения $u_{k}^{*}, u_{s k}^{*}$ на промежутках $\left[t_{k}, t_{k}+h\right]$ :

$$
\begin{aligned}
& u^{*}=\left\{u_{k}^{*}, t \in\left[t_{k}, t_{k}+h\right]\right\} ; u^{*}=\left(u_{v}^{*}, u_{s}^{*}\right) ; u_{k}^{*}=\left(u_{k}^{*}, u_{S k}^{*}\right) ; \\
& t_{k}=k h ; k=\overline{0, \sigma-1} ; \sigma h=T .
\end{aligned}
$$

Построение $u_{k}^{*}$ в каждой позиции $\left(t_{k}, Q_{u}\left(X, t_{k}\right)\right)$ по существу формирует закон управления с обратной связью $u_{k}^{* *}\left(t_{k}, Q_{u}\left(X, t_{k}\right)\right.$. Если вычисление $u_{k}^{*}$ производится за время, не превышающее достаточно малого периода квантования $\mathrm{h}$, то этот закон реализуется с шагом $\mathrm{h}$ :

$$
u_{k}^{*}\left(t_{k}+h\right)=u_{k}^{* *}\left(t_{k}, Q_{u}\left(X, t_{k}\right)\right)
$$

обеспечивая тем самым последовательную отработку алгоритма (42) в реальном времени. При этом в условиях $\mathrm{h} \rightarrow 0$ получаем в соответствии с (42), (43):

$$
\mathrm{U}^{*}=\mathrm{U}^{* *}\left(\tau, \mathrm{Q}_{\mathrm{u}}(\mathrm{X}, \tau)\right) \forall \tau \in(0, \mathrm{~T}) .
$$

Дальнейшая процедура $\Delta_{\Sigma}^{\left(\mathrm{N}_{\mathrm{k}}\right)}-$ и $\Delta_{\Sigma}^{(\mathrm{N})}$ - параметризации искомых управлений $\mathrm{u}_{\mathrm{k}}^{*}$ и $\mathrm{u}^{*}$ соответственно приводит по предлагаемой общей методологии к последовательному решению с шагом h ЗПО вида (41)

$$
\begin{aligned}
& \mathrm{I}_{1 \mathrm{k}}\left(\Delta_{\Sigma}^{\left(\mathrm{N}_{\mathrm{k} 0}\right)}, \mathrm{t}_{\mathrm{k}}\right)=\max _{\mathrm{Q}_{0}, \mathrm{w}_{1 \mathrm{k}}, \mathrm{w}_{2 \mathrm{k}}} \mathrm{I}_{\mathrm{k}}\left(\Delta_{\Sigma}^{\left(\mathrm{N}_{\mathrm{k} 0}\right)}, \mathrm{Q}_{0}, \mathrm{t}_{\mathrm{k}}, \mathrm{W}_{1 \mathrm{k}}, \mathrm{W}_{2 \mathrm{k}}\right) \rightarrow \min _{\Delta_{\Sigma}^{\left(\mathrm{N}_{0}\right)} \in \Omega_{\mathrm{N}_{\mathrm{k} 0}}} ; \\
& \Phi_{1 \mathrm{k}}\left(\Delta_{\Sigma}^{\left(\mathrm{N}_{\mathrm{k} 0}\right)}, \mathrm{t}_{\mathrm{k}}\right)=\max _{\mathrm{Z}_{\mathrm{k}} \in \mathrm{Z}_{1 \mathrm{k}}}\left|\mathrm{Q}\left(\mathrm{Z}_{1 \mathrm{k}}, \Delta_{\Sigma}^{\left(\mathrm{N}_{0 \mathrm{k}}\right)}, \mathrm{t}_{\mathrm{k}}\right)-\mathrm{Q}^{* *}(\mathrm{X})\right| \leq \varepsilon^{(\mathrm{k})} ; \\
& \mathrm{k}=\overline{0, \sigma-1} ; \mathrm{Z}_{1 \mathrm{k}}=\left(\mathrm{X}, \mathrm{Q}_{0}, \mathrm{~W}_{1 \mathrm{k}}, \mathrm{W}_{2 \mathrm{k}}\right) ; \mathrm{Z}_{1 \mathrm{k}}=\mathrm{V} \times \mathrm{W}_{2 \mathrm{k}} ; \mathrm{W}_{2 \mathrm{k}}=\left(\mathrm{Q}_{0}, \mathrm{~W}_{1 \mathrm{k}}, \mathrm{W}_{2 \mathrm{k}}\right) \in \mathrm{W}
\end{aligned}
$$

с зависящим от $t_{k}$ континуумом ограничений $\Phi_{1 k}\left(\Delta_{\Sigma}^{\left(\mathrm{N}_{k 0}\right)}, t_{k}\right)$ на конечное состояние объекта при последовательном уменьшении влияния на него интегрального эффекта воздействия возмущений $\mathrm{w}_{1 k}, \mathrm{w}_{2 \mathrm{k}} \in \mathrm{W}_{2 \mathrm{k}}$, на промежутках $\mathrm{t} \in\left[\mathrm{t}_{\mathrm{k}}, \mathrm{T}\right]$ при $\mathrm{t}_{\mathrm{k}} \rightarrow \mathrm{T}$. Здесь явные зависимости $\mathrm{Q}\left(\mathrm{z}_{1 \mathrm{k}}, \Delta_{\Sigma}^{\left(\mathrm{N}_{\mathrm{k} 0}\right)}, \mathrm{t}_{\mathrm{k}}\right)$ и $\mathrm{I}_{\mathrm{k}}\left(\Delta_{\Sigma}^{\left(\mathrm{N}_{\mathrm{k} 0}\right)}, \mathrm{Q}_{0}, \mathrm{t}_{\mathrm{k}}, \mathrm{w}_{1 \mathrm{k}}, \mathrm{w}_{2 \mathrm{k}}\right)$ от своих аргументов должны быть получены после интегрирования уравнений модели объекта (1)-(3) с измеряемым начальным состоянием $\mathrm{Q}\left(\mathrm{X}, \mathrm{t}_{\mathrm{k}}\right)=\mathrm{Q}_{\mathrm{u}}\left(\mathrm{X}, \mathrm{t}_{\mathrm{k}}\right)$ вместо (3) при $\Delta^{\left(\mathrm{N}_{\mathrm{k}}\right)}$-параметризованном управлении $\mathrm{u}_{\mathrm{k}}^{*}$. В соответствии с требованием (39) ошибки равномерного приближения $\varepsilon^{(\mathrm{k})}, \mathrm{k}=\overline{0, \sigma-1}$, в (45) должны выбираться таким образом, чтобы обеспечить выполнение соотношения $\varepsilon^{(\sigma-1)} \leq \varepsilon$. По найден- 
ным оптимальным значениям $\bar{\Delta}_{\Sigma}^{\left(\mathrm{N}_{\mathrm{k} 0}\right)}$ вычисляются и реализуются на объекте, согласно алгоритму (42), параметры $\bar{\Delta}_{\Sigma}^{\left(\mathrm{N}_{0}\right)}$ оптимальных управлений $u_{\mathrm{V}}^{*}$ и $\mathrm{u}_{\mathrm{S}}^{*}$.

\section{7 Оптимальное проектирование объектов технологической теплофизики}

В качестве примера, представляющего самостоятельный интерес, рассмотрим детерминированную задачу оптимального проектирования установки градиентного индукционного нагрева (ИНУ) цилиндрических слитков из алюминиевых сплавов перед последующей операцией прессования в ответственных технологических комплексах обработки металла давлением [16].

Температурное поле $\mathrm{Q}\left(\mathrm{x}_{1}, \mathrm{x}_{2}\right)$ в процессе индукционного нагрева описывается в зависимости от времени $t \in[0, T]$, радиальной $x_{1}$ и продольной $x_{2}$ координат в области $\mathrm{V}=\left(\mathrm{x}_{1} \in[0,1], \mathrm{x}_{2} \in[0,1]\right)$ двумерным неоднородным уравнением теплопроводности в относительных единицах вида (1) с начальными и граничными условиями (2), (3) третьего рода и заданным законом пространственного распределения внутренних источников тепла, определяемым известным решением уравнений Максвелла для электромагнитного поля индуктора.

Градиентный нагрев слитка с положительным перепадом конечной температуры $\mathrm{Q}\left(\mathrm{x}_{1}, \mathrm{x}_{2}, \mathrm{~T}\right)$ по его длине $\mathrm{x}_{2}$ в направлении прессования, который обеспечивает существенное повышение производительности пресса, осуществляется в ИНУ двухсекционного исполнения (рисунок 1) [16]. В качестве внутренних сосредоточенных УВ $u_{v}(t)$ используются суммарные удельные мощности внутреннего тепловыделения в обеих секциях $\mathrm{v}_{\mathrm{v}}(\mathrm{t})=\left(\mathrm{v}_{1}(\mathrm{t}), \mathrm{v}_{2}(\mathrm{t})\right)$, подчиненные ограничениям вида (8):
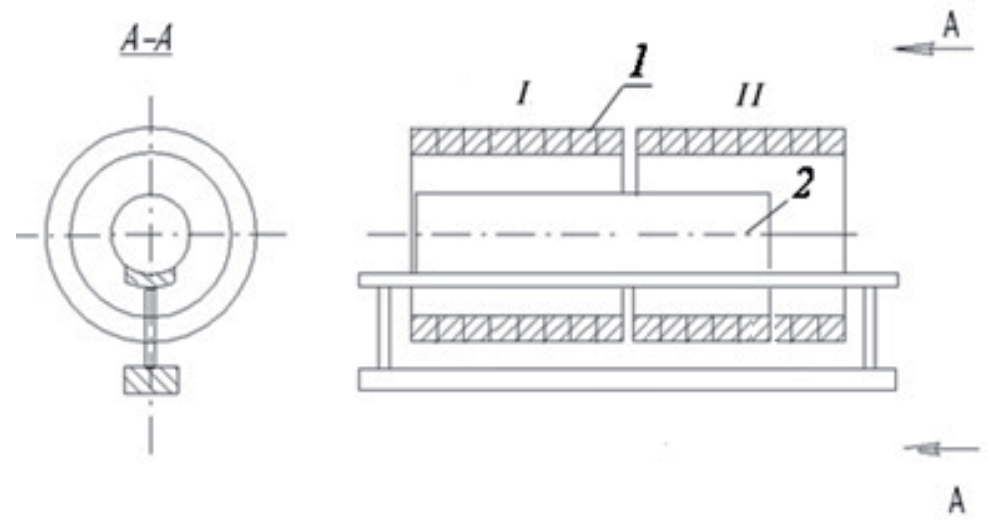

Рисунок 1 - Конструкция двухсекционной ИНУ периодического действия: 1- секция индуктора; 2- заготовка

$$
0 \leq \mathrm{u}_{1}(\mathrm{t}) \leq \mathrm{u}_{\mathrm{v}_{\text {max }}} ; 0 \leq \mathrm{u}_{2}(\mathrm{t}) \leq \mathrm{u}_{2 \text { max }} .
$$

В роли искомых базовых проектных решений ИНУ заданной двухсекционной конструкции будем рассматривать максимальные мощности секций $u_{1 \text { max }}$ и $u_{2 \text { max }}$, стесняющие возможные пределы изменения режимов УВ в (46), и, следовательно, в данном случае будем иметь для $g(X)$ в (4):

$$
g(X)=\left(u_{1 \text { max }}, u_{2 \text { max }}\right)=\text { const. }
$$

В качестве критерия оптимальности в условиях заданной длительности процесса нагрева фиксируемой требуемой производительностью технологического комплекса «ИНУ-пресс» 
принимается, подобно (11), достижимая точность равномерного приближения конечного температурного состояния к его заданному неравномерному распределению перед последующим прессованием слитка:

$$
I^{*}=\max _{x_{1}, x_{2} \in[0,1]}\left|Q\left(x_{1}, x_{2}, T, u_{v_{1}}(t), u_{v_{2}}(t), u_{v_{1 m a x}}, u_{v_{2} \max }\right)-Q^{* *}\left(x_{1}, x_{2}\right)\right| \rightarrow \min _{u_{v}, g} .
$$

Требования к градиентному характеру нагрева слитка приводят к заданию $\mathrm{Q}^{* *}\left(\mathrm{x}_{1}, \mathrm{x}_{2}\right)$ в форме кусочно-линейной функции продольной координаты

$$
\mathrm{Q}^{* *}\left(\mathrm{x}_{1}, \mathrm{x}_{2}\right)=\left\{\begin{array}{l}
\mathrm{Q}_{*}^{(1)}, 0 \leq \mathrm{x}_{2} \leq \mathrm{x}_{2}^{*} ; \\
\mathrm{Q}_{*}^{(1)}+\frac{\mathrm{Q}_{*}^{(2)}-\mathrm{Q}_{*}^{(1)}}{1-\mathrm{x}_{2}^{*}}\left(\mathrm{x}_{2}-\mathrm{x}_{2}^{*}\right), \mathrm{x}_{2}^{*} \leq \mathrm{x}_{2} \leq 1
\end{array}\right.
$$

с известными постоянными $\mathrm{Q}_{*}^{(1)}, \mathrm{Q}_{*}^{(2)}, \mathrm{x}_{2}^{*}$.

Проблема теперь сводится к совместному поиску оптимальных управлений $u_{1}^{*}(t), u_{2}^{*}(t)$,

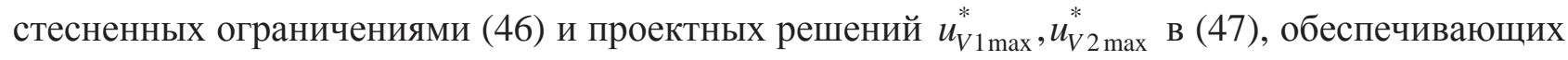
в совокупности достижение минимального значения критерия оптимальности (48).

Данная задача является частным случаем общей детерминированной задачи (31) оптимального проектирования СРП с целевой функцией вида (11).

Известные условия оптимальности в форме принципа максимума Понтрягина заведомо определяют $u_{v_{1}}^{*}(t)$ и $u_{v_{2}}^{*}(t)$ в виде кусочно-постоянных функций времени, попеременно принимающих только свои предельно допустимые значения согласно (46) $[1,8,9]$. Тем самым обеспечивается $\Delta^{(\mathrm{N})}$-параметризация искомых УВ с точностью до числа $\mathrm{N}$ и длительностей $\Delta_{\mathrm{i}}^{(\mathrm{N})}, \mathrm{i}=\overline{1, \mathrm{~N}}$, интервалов их постоянства на протяжении процесса управления в условиях свободы выбора $T$ из условий достижения минимальной величины критерия оптимальности (48). В наиболее просто реализуемых режимах работы ИНУ с синхронным управлением во времени обеими секциями один и тот же вектор $\Delta^{(\mathrm{N})}$ характеризует одновременно оба воздействия $u_{1}^{*}$ и $u_{2}^{*}$.

При $\mathrm{T}=\sum_{\mathrm{i}=1}^{\mathrm{N}} \Delta_{\mathrm{i}}^{(\mathrm{N})}$ для простейшего по условиям технической реализации изначально задаваемого режима работы ИНУ с неизменной во времени максимальной мощностью обеих секций будем иметь $\mathrm{N}_{0}=1$, и вектор $\Delta^{\left(\mathrm{N}_{0}\right)}=\Delta_{1}^{(1)}$ тем самым определён с точностью до длительности $\mathrm{T}=\Delta_{1}^{(1)}$ процесса управления.

$\Delta_{\mathrm{g}}^{\left(\mathrm{N}_{\mathrm{g} 0}\right)}$-параметризация искомых проектных решений $\mathrm{g}(\mathrm{X})$ в данном случае непосредственно описывается их заданием в форме (47) при $\mathrm{N}_{\mathrm{g} 0}=2$ :

$$
\Delta_{\mathrm{g}}^{\left(\mathrm{N}_{\mathrm{g} 0}\right)}=\Delta_{\mathrm{g}}^{(2)}=\left(\Delta_{\mathrm{g} 1}^{(2)}, \Delta_{\mathrm{g} 2}^{(2)}\right) ; \Delta_{\mathrm{g} 1}^{(2)}=\mathrm{u}_{1 \text { max }} ; \Delta_{\mathrm{g} 2}^{(2)}=\mathrm{u}_{22 \max } \cdot
$$

В итоге получаем для совместного вектора искомых параметров $\Delta_{\Sigma}^{\left(\mathrm{N}_{0}+\mathrm{N}_{\mathrm{g} 0}\right)}$ :

$$
\mathrm{N}_{0}+\mathrm{N}_{\mathrm{g} 0}=3 ; \Delta_{\Sigma}^{\left(\mathrm{N}_{0}+\mathrm{N}_{\mathrm{g} 0}\right)}=\Delta_{\Sigma}^{(3)}=\left(\Delta_{1}^{(1)}, \mathrm{u}_{1 \max }, \mathrm{u}_{2 \max }\right)
$$


Интегрирование (в аналитической или численной форме) уравнений модели при параметризованных управлениях $u_{v}\left(t, \Delta_{1}^{(1)}\right), g(X)=\left(u_{1 \max }, u_{2 \max }\right)$ с последующей подстановкой результатов в (48) обеспечивают редукцию к задаче вида (32) без ограничений с целевой функцией, подобной (25) и (30):

$$
\begin{aligned}
& \mathrm{I}_{1}^{*}\left(\Delta_{\Sigma}^{\left(\mathrm{N}_{0}+\mathrm{N}_{\mathrm{g} 0}\right)}\right)=\max _{\mathrm{x}_{1}, \mathrm{x}_{2} \in[0,1]}\left|\mathrm{Q}\left(\mathrm{X}_{1}, \mathrm{X}_{2}, \Delta_{\Sigma}^{\left(\mathrm{N}_{0}+\mathrm{N}_{\mathrm{g} 0}\right)}\right)-\mathrm{Q}^{* *}\left(\mathrm{X}_{1}, \mathrm{X}_{2}\right)\right|= \\
& =\max _{x_{1}, x_{2} \in[0,1]}\left|Q\left(x_{1}, x_{2}, \Delta_{1}^{(1)}, u_{v_{1} \max }, u_{v_{2} \max }\right)-Q^{* * *}\left(x_{1}, x_{2}\right)\right| \rightarrow \underset{\Delta_{1}^{(1)}, u_{V_{1} \max }, u_{v_{2} \max }}{\min } .
\end{aligned}
$$

Альтернансные свойства искомых решений $\bar{\Delta}_{\Sigma}^{\left(\mathrm{N}_{0}+\mathrm{N}_{\mathrm{g} 0}\right)}=\left(\bar{\Delta}_{1}^{(1)}, \mathrm{q}_{1 \mathrm{max}}, \mathrm{q}_{2 \mathrm{max}}\right)$, определяемые в рассматриваемом случае подобно задаче (25) базовыми соотношениями (22), (24) (где надо заменить $\bar{\Delta}^{\left(\mathrm{N}_{0}\right)}$ на $\left.\bar{\Delta}_{\Sigma}^{\left(\mathrm{N}_{0}+\mathrm{N}_{\mathrm{g} 0}\right)}\right)$, приводят с использованием известных закономерностей конечного пространственного температурного распределения $[8,9,16]$ к следующей системе шести уравнений альтернансного метода, разрешаемой известными способами относительно всех шести искомых параметров оптимального процесса нагрева $\bar{\Delta}_{1}^{(1)}, \overline{\mathrm{u}}_{1 \max }, \overline{\mathrm{u}}_{2 \max }$ $\varepsilon_{\min }^{(3)}=\min _{1}^{*}, \mathrm{X}_{4}^{0}=\left(\mathrm{X}_{1 \mathrm{e}}^{0}, \mathrm{x}_{2 \mathrm{e}}^{0}\right)$ включая выступающие в роли промежуточных неизвестных координаты точки экстремума $X_{4}^{0}$ :

$$
\begin{aligned}
& \mathrm{Q}\left(\mathrm{X}_{1}^{0}, \bar{\Delta}_{1}^{(1)}, \overline{\mathrm{u}}_{1 \max }, \overline{\mathrm{u}}_{2 \max }\right)-\mathrm{Q}^{* *}\left(\mathrm{X}_{1}^{0}\right)=-\varepsilon_{\min }^{(3)} \text {; } \\
& \mathrm{Q}\left(\mathrm{X}_{2}^{0}, \bar{\Delta}_{1}^{(1)}, \overline{\mathrm{u}}_{1 \max }, \overline{\mathrm{u}}_{2 \max }\right)-\mathrm{Q}^{* *}\left(\mathrm{X}_{2}^{0}\right)=-\varepsilon_{\min }^{(3)} \text {; } \\
& \mathrm{Q}\left(\mathrm{X}_{3}^{0}, \bar{\Delta}_{1}^{(1)}, \overline{\mathrm{u}}_{\mathrm{V}_{\max }}, \overline{\mathrm{u}}_{2 \max }\right)-\mathrm{Q}^{* *}\left(\mathrm{X}_{3}^{0}\right)=-\varepsilon_{\min }^{(3)} \text {; } \\
& \mathrm{Q}\left(\mathrm{X}_{4}^{0}, \bar{\Delta}_{1}^{(1)}, \overline{\mathrm{u}}_{1 \max }, \overline{\mathrm{u}}_{2 \max }\right)-\mathrm{Q}^{* *}\left(\mathrm{X}_{4}^{0}\right)=\varepsilon_{\min }^{(3)} \text {; } \\
& \frac{\partial}{\partial \mathbf{X}_{1}}\left(\mathrm{Q}\left(\mathrm{X}_{4}^{0}, \bar{\Delta}_{1}^{(1)}, \overline{\mathrm{u}}_{1 \mathrm{max}}, \overline{\mathrm{u}}_{2 \max }\right)-\mathrm{Q}^{* *}\left(\mathrm{X}_{4}^{0}\right)\right)=0 \\
& \frac{\partial}{\partial \mathbf{X}_{2}}\left(\mathrm{Q}\left(\mathbf{X}_{4}^{0}, \bar{\Delta}_{1}^{(1)}, \overline{\mathbf{u}}_{1 \max }, \overline{\mathbf{u}}_{v_{\text {max }}}\right)-\mathrm{Q}^{* *}\left(\mathbf{X}_{4}^{0}\right)\right)=0 \text {; } \\
& X_{j}^{0}=\left(x_{1 j}^{0}, x_{2 j}^{0}\right), j=1,4 ; x_{1 j}^{0} \in\left\{x_{1}: 0 \leq x_{1} \leq 1\right\} ; x_{2 j}^{0} \in\left\{x_{2}: 0 \leq x_{2} \leq 1\right\} ; \\
& \mathrm{X}_{1}^{0}=(0,0) ; \mathrm{X}_{2}^{0}=(0,1) ; \mathrm{X}_{3}^{0}=(1,1) ; \mathrm{X}_{4}^{0}=\left(\mathrm{X}_{\mathrm{le}}^{0}, \mathrm{X}_{2 \mathrm{e}}^{0}\right) \text {; } \\
& 0<\mathrm{x}_{1 \mathrm{e}}^{0}<1 ; 0<\mathrm{x}_{2 \mathrm{e}}^{0}<1 \text {. }
\end{aligned}
$$

Некоторые расчётные результаты, полученные при нагреве цилиндрических слитков из алюминиевых сплавов Д16 (АА2024) в ИНУ двухсекционного исполнения при $\mathrm{T}=215^{\circ} \mathrm{C}$, приведены на рисунке 2.

\section{Заключение}

Предлагается технология построения программной стратегии принятия решений применительно к широкому кругу задач оптимального проектирования сложноструктурированных систем с распределёнными параметрами, трактуемых как комплексная проблема поиска проектных решений и последующих режимов функционирования объекта, которые обеспечивают в совокупности достижение экстремальных значений совместного критерия качества.

Развиваемая в статье методология базируется на предварительных процедурах параметризации УВ, операции точной редукции исходной задачи к специальным задачам математического программирования и конструктивных способах их последующего решения с исполь- 
зованием альтернансных свойств искомых экстремалей и фундаментальных закономерностей конкретной ПрО.

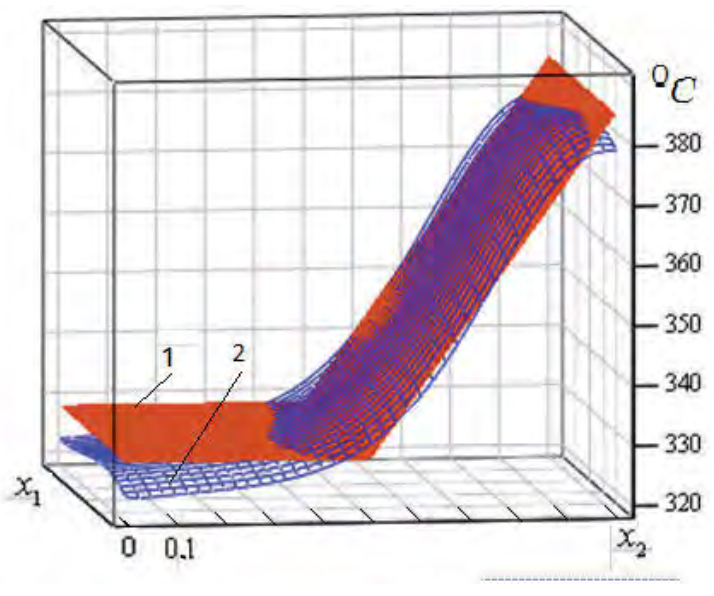

$a$

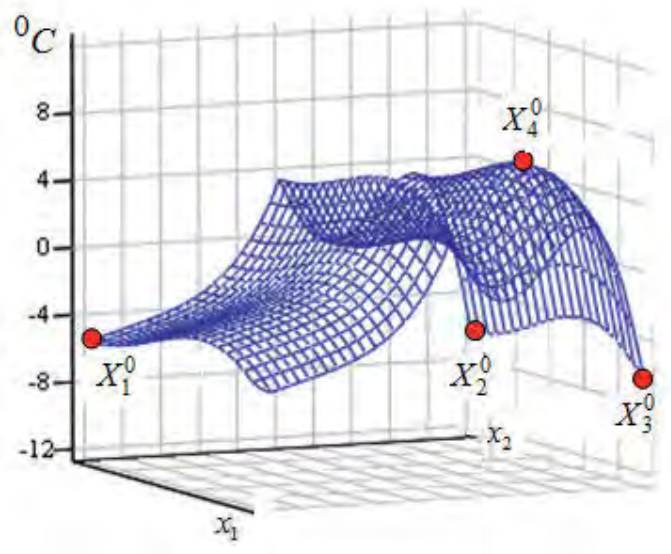

Рисунок 2 - Заданное (1) и полученное (2) температурные распределения по объёму заготовки в процессе нагрева в ИНУ $(a)$, отклонение полученного температурного распределения от заданного по объему (в), радиусу (2) и длине (б) заготовки для

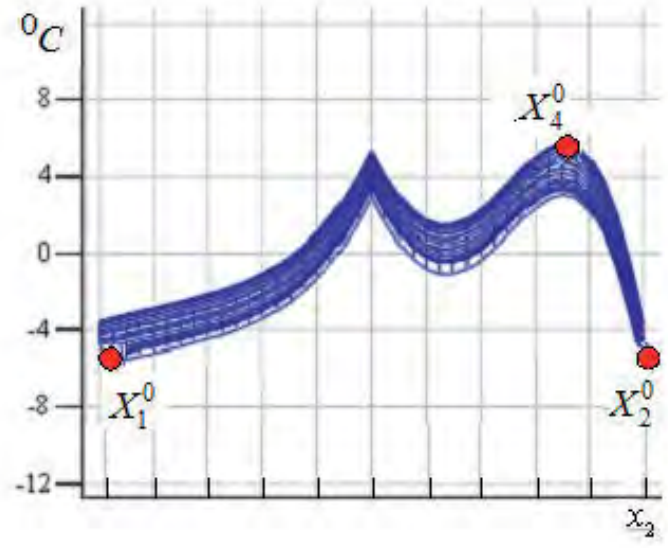

$\sigma$

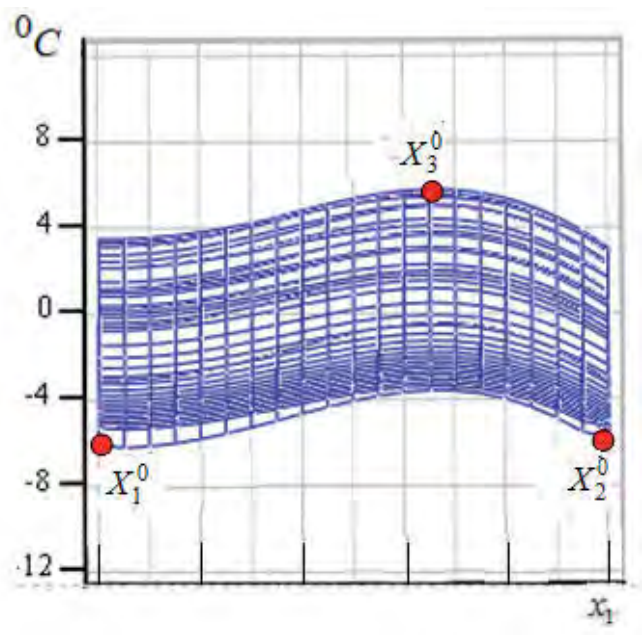

2

\section{Список источников}

[1] Бутковский, А.Г. Теория оптимального управления системами с распределенными параметрами / А.Г. Бутковский. - М.: Наука, 1965. - 474 с.

[2] Бутковский, $\boldsymbol{A . \Gamma . ~ М е т о д ы ~ у п р а в л е н и я ~ с и с т е м а м и ~ с ~ р а с п р е д е л е н н ы м и ~ п а р а м е т р а м и / ~ А . Г . ~ Б у т к о в с к и и ̆ . ~ - ~ М . : ~}$ Наука, 1975. - 568 с.

[3] Бутковский, А.Г. Оптимальное управление нагревом металла / А.Г. Бутковский, С.А. Малый, Ю.Н. Андреев. - М.: Металлургия, 1972. - 440 с.

[4] Лионс, Ж-Л. Оптимальное управление системами, описываемыми уравнениями с частными производными / Ж.-Л. Лионс. - М.: Мир, 1972. - 414 с.

[5] Лурье, К.A. Оптимальное управление в задачах математической физики / К.А. Лурье. - М.: Наука, 1975. $478 \mathrm{c.}$

[6] Сиразетдинов, T.К. Оптимизация систем с распределенными параметрами / Т.К. Сиразетдинов. - М.: Наука, 1977. - 480 с. 
[7] Фурсиков, А.B. Оптимальное управление распределенными системами. Теория и приложения / А.В. Фурсиков. - Новосибирск: Научная книга, 1999. - 350 с.

[8] Panonopm,Э.Я. Оптимальное управление системами с распределенными параметрами / Э.Я. Рапопорт. М.: Высшая школа, 2009. - 677 с.

[9] Panonopm,Э.Я. Альтернансный метод в прикладных задачах оптимизации / Э.Я. Рапопорт. - М.: Наука, 2000. - $336 \mathrm{c}$.

[10] Плешивцева, Ю.Э. Метод последовательной параметризации управляющих воздействий в краевых задачах оптимального управления системами с распределенными параметрами / Ю.Э. Плешивцева, Э.Я. Рапопорт // Известия РАН. Теория и системы управления. - 2009. - №3. - С. 22-33.

[11] Куржанский, А.Б. Управление и наблюдение в условиях неопределенности / А.Б. Куржанский. - М.: Наука, 1977. - 392 с.

[12] Габасов, Р. Оптимальное управление и наблюдение в реальном времени / Р. Габасов, Ф.М. Кириллова // Известия РАН. Теория и системы управления. - 2006. - №3. - С. 90-111.

[13] Габасов, P. Реализация в реальном времени оптимальных обратных связей по выходу для линейных систем в условиях неопределенности / Р. Габасов, Ф.М. Кириллова, Т.И. Песецкая // Известия РАН. Теория и системы управления. - 2005. - №4. - С. 44-56.

[14] Коллати,Л. Теория приближений. Чебышевские приближения / Л. Колатц, В. Крабс. - М.: Наука, 1978. $271 \mathrm{c.}$

[15] Карлин, С. Чебышевские системы и их применение в анализе и статистике / С. Карлин, В. Стадден. - М.: Наука, 1976. - 568 с.

[16] Panonopm,Э.Я. Оптимальное управление температурными режимами индукционного нагрева / Э.Я. Рапопорт, Ю.Э. Плешивцева. - М.: Наука, 2012. - 309 с.

\title{
TECHNOLOGY OF OPTIMAL DESIGNING OF COMPLEX STRUCTURED SYSTEMS WITH DISTRIBUTED PARAMETERS: PROGRAM STRATEGY OF DECISION MAKING
}

\author{
E.Ya. Rapoport, Yu.E. Pleshivtseva \\ Institute of C ontrol of Complex Systems, RAS, Samara, Russia \\ edgar.rapoport@mail.ru
}

\begin{abstract}
This article describes the statements and suggests constructive technology for solving the wide class of problems of joint optimization of design and operating modes for complex structured, dimensionless, not well-determined systems with distributed parameters (SDP). The optimization problems are formulated with respect to complex efficiency criteria. SDP are described with operator equations of the general form with the appropriate initial and boundary conditions. As a basic problem, the deterministic problem of optimal design is considered to find program control of operational modes for distributed system with well-determined object characteristics. Suggested method for the problem solution is extended to the optimization problems for design solutions under conditions of a priori known external inputs on the object. The method is also applied to the complex problem of the joint optimization and to the typical cases of optimal design of the distributed system under conditions of interval uncertainty of the system's parametric characteristics and external disturbances. Developed approach uses: the procedure of subsequent parameterization of problems' solutions that is based on well-known analytical conditions of optimality; precise reduction to the special problems of mathematical programming; alternance properties of extremum solutions that are similar to well-known results of theory of nonlinear Chebyshev's approximations; and fundamental regularities of the subject domain. The given example of an application of the received results to the optimal design problem solution for industrial object of the technological thermal physics is of a special interest. The formulation of the problem of distributed parameters system design as a complex problem of joint determination of the vector space-time control actions is new. The approach for development of program strategies of the decision making that can be extended to the considered problems class is also new.
\end{abstract}

Key words distributed parameters system, optimal design, semi-infinite optimization, alternance method. 
Citation: Rapoprt EYa, Pleshivtseva YuE. Technology of optimal designing of complex structured systems with distributed parameters: Program strategy of decision making. Ontology of designing. 2017; 7(2): 172-190. DOI: 10.18287/2223-9537-2017-7-2-172-190.

\section{References}

[1] Butkovsky AG. Theory of optimal control of systems with distributed parameters [In Russian]. - Moscow: Nauka; 1965.

[2] Butkovsky AG. Methods of control of systems with distributed parameters [In Russian]. - Moscow: Nauka; 1975.

[3] Butkovsky AG. Optimal control of metal heating металла [In Russian]. - Moscow: Nauka; 1972.

[4] Lions Zh.-L. Optimal control of systems described by equations in partial derivatives [In Russian]. - Moscow: Mir; 1972.

[5] LurjeKA Optimal control in problems of mathematical physics [In Russian]. - Moscow: Nauka; 1972.

[6] SirazetdinovTK. Optimization of distributed parameters systems [In Russian]. - Moscow: Nauka; 1977.

[7] Fursikov AV. Optimal control of distributed systems. Theory and applications [In Russian]. - Moscow: Nauchnaya kniga; 1999.

[8] Rapoport EYa Optimal control of systems with distributed parameters [In Russian]. - Moscow: Vysshaya shkola; 2009.

[9] RapoportEYa Alternance method in applied optimization problems [In Russian]. - Moscow: Nauka; 2000.

[10] Pleshivtseva YuE., Rapoport EYa. Method of subsequent parameterization of controlling actions in boundary problems of optimal control of systems with distributed parameters [In Russian]. Izvestiya RAN. Theory and control systems 2009; №3, P. 22-33.

[11] Kurzhanskyj AB. Control and observation under conditions of uncertainty [In Russian]. - Moscow: Nauka; 1977.

[12] Gabasov R., Kirillova F. Optimal control and observation in real time. Izvestiya RAN. Theory and control systems 2006; №3, P. 90-111.

[13] Gabasov R., Kirillova F. Real time implementation of optimal output' feed backs in linear systems under conditions of uncertainty. Izvestiya RAN. Theory and control systems 2005; №5, P. 44-56.

[14] Kollats L., Krabs V. Theory of approximations. Chebyshevs' approximations [In Russian]. - Moscow: Nauka; 1978.

[15] Karlin S., Stadden V. Chebyshevs' systems and their application in analysis and statistics [In Russian]. - Moscow: Nauka; 1976.

[16] RapoportE., Pleshivtseva Yu. Optimal control of temperature modes of induction heating [In Russian]. - Moscow: Nauka, 2012.

\section{Сведения об авторах}

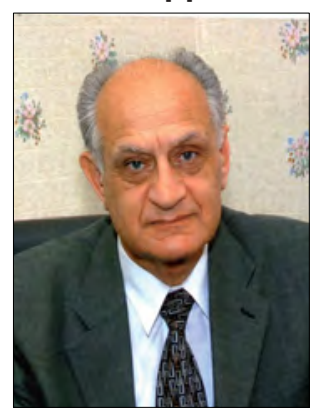

Рапопорт Эдгар Яковлевич, 1936 г. рождения. Окончил Куйбышевский индустриальный институт в 1959 г., д.т.н. (1984). Профессор кафедры «Автоматика и управление в технических системах» Самарского государственного технического университета, заслуженный деятель науки и техники РФ. В списке научных трудов 8 монографий и более 350 работ в области теории моделирования и оптимального управления системами с распределёнными параметрами.

Edgar Yakovevich Rapoport (b. 1936) graduated from the Kuibushev Polytechnic Institute (Kuibyshev-city) in 1959, Doctor of Science (1984). Hi is Professor at Samara State Technical University (Department of automatics and control in technical systems), Honoured Worker of science and technology of Russian Federation. He has 8 monographs and more than 350 scientific publications in the field of simulation and optimal control of systems with distributed parameters.

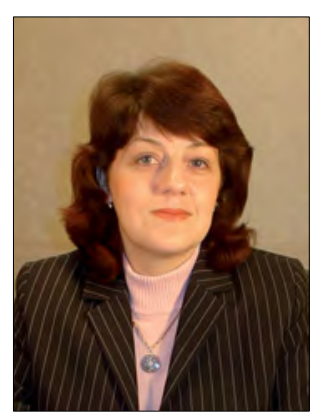

Плешивцева Юлия Эдгаровна, 1965 г. рождения. Окончила Куйбышевский политехнический институт им. В.В. Куйбышева в 1987 г., д.т.н. (2009). Профессор кафедры «Управление и системный анализ теплоэнергетических и социотехнических комплексов» Самарского государственного технического университета, почетный работник высшего образования РФ. В списке научных трудов 3 монографии и более 150 публикаций в области теории оптимального управления системами с распределёнными параметрами.

Yuliya Edgarovna Pleshivtseva (b. 1965) graduated from the Kuibushev Polytechnic Institute (Kuibyshev-city) in 1987, Doctor of Science (2009). She is Professor at Samara State Technical University (Department of Control and System Analysis in Heat-and-Power Engineering), coauthor of 3 monographs and more than 150 publications in the field of theory of optimal control of systems with distributed parameters. 


\title{
УДК 629.7
}

\section{ПРОЕКТИРОВАНИЕ СИЛОВЫХ АДДИТИВНЫХ КОНСТРУКЦИЙ: TЕОРЕТИЧЕСКИЕ ОСНОВЫ}

\author{
В.А. Комаров \\ Самарский национальный исследовательский университет имени академика С.П. Королева, Самара, Россия \\ vkomarov@ssau.ru
}

\begin{abstract}
Аннотация
Рассматриваются взаимосвязи и взаимное влияние в триаде основных инженерных понятий - материал, технология, конструкция. Обсуждаются возможности аддитивных технологий по созданию новых конструкций и материалов. Ставится и решается задача об оптимальном распределении материала в теле с переменной плотностью. Предлагается алгоритм топологической оптимизации силовых конструкций из материала с переменной плотностью по критерию минимума массы. Основные механические свойства гипотетического материала предполагаются пропорциональными его плотности. Рассматриваются ограничения по прочности и жёсткости при одном и нескольких случаях нагружения. Обсуждается и уточняется содержание понятий «оптимальная» и «рациональная» конструкция. Предлагается объективный критерий «силовой фактор», который учитывает величину и протяжённость действия внутренних усилий, для сравнения весового совершенства различных вариантов конструкций и оценки их близости к теоретически оптимальному решению. Приводятся примеры проектирования плоских и пространственных конструкций. Как одно из возможных направлений дальнейшего развития аддитивных технологий формулируются задачи по разработке новых материалов и способов создания структур с переменной плотностью.
\end{abstract}

Ключевые слова: аддитивные технологии, проектирование, конструкция, прочность, жёсткость, материал, оптимизация, тело переменной плотности.

Цитирование: Комаров, В.А. Проектирование силовых аддитивных конструкций: теоретические основы / В.А. Комаров // Онтология проектирования. - 2017. - Т. 7, №2(24). - С. 191-206. - DOI: 10.18287/2223-9537-2017-7-2-191-206.

\section{Введение}

Значительную часть искусственной окружающей среды составляют конструкции различного назначения. При разработке многих из них предъявляются требования достаточной прочности и жёсткости при минимуме массы. На протяжении всего развития цивилизации решение таких задач находится в триаде: материал - технология - конструкция. В зависимости от важности или новизны одного из этих компонентов устанавливается классификация конструкций: каменные, металлические, литые, клёпаные, сварные, тонкостенные и другие. Каждый из этих классов конструкций имеет свои специфические методы проектирования.

Совсем недавно появился принципиально новый метод создания конструкций - аддитивные технологии $[1,2]$. Возможности этого метода и отдельных направлений его развития многообразны и сулят большой технический эффект [3]. Метод позволяет изготавливать конструкции из самых различных материалов: полимеров, металлов, керамики и всевозможных комбинаций из них [4]. Стало возможным производство монолитных конструкций с внутренними полостями, стержневыми структурами внутри оболочек и т.д.

Возникает вопрос: как наилучшим образом использовать эти новые возможности? На первых порах практическое внедрение аддитивных технологий началось с повторного изготовления существующих конструкций сложных форм, которые были ранее спроектированы 
по традиционным методам из изотропных материалов. Этот подход позволяет отказаться от таких сложных производственных технологий, как штамповка, литьё, сварка, механическая обработка и т.п. Однако, при этом новые технологические возможности аддитивных методов не используются в полной мере. Одна из причин такого положения состоит в том, что быстрое развитие аддитивных технологий не обеспечено должным образом теоретическими разработками. Здесь уместно напомнить известное положение из диалектики: «Теория без практики мертва, но теория указывает путь практике».

Возможности аддитивных технологий позволяют задуматься о создании сверхлёгких конструкций практически без оглядки на технологические ограничения. Поэтому, с точки зрения теории, интересно знать, как выглядит предел совершенства конструкций в каждом конкретном случае: при заданных геометрических ограничениях на размещение конструкции, условиях закрепления и действующих внешних воздействиях.

\section{1 Задачи проектирования конструкций}

В проектировании конструкций полезно выделить два основных вида деятельности: выбор структуры конструкции и выбор рациональных размеров её элементов. Первый вид проектной деятельности в русскоязычной литературе называется «выбор силовой схемы». Второй вид называется «параметрическая оптимизация» в связи с удобством формализации задач в терминах нелинейного математического программирования. Силовая схема предопределяет пути передачи сил. Назначение рациональных размеров элементов конструкции направлено на обеспечение условий прочности и жёсткости при минимальном расходе материала. В последнее время первый вид деятельности под влиянием зарубежной технической литературы стал обозначаться термином topology optimization - «топологическая оптимизация», а второй вид - sizing - «образмеривание».

В проектировании силовых конструкций до последнего времени существенную роль играет накопленный опыт и инженерная интуиция. Особенно это касается выбора структуры. Практика выработала два принципа проектирования, направленных на создание эффективных конструкций.

Первыци приниип - передача усилий по кратчайшим путям. Иногда это очень просто. Например, на рисунке 1а показана передача сосредоточенной силы $P$ к заделке. Оптимальное решение - стержень, соединяющий точки А и В. При изменении направления силы $P$ на некоторый угол, например, на 90, выбор оптимальной структуры не очевиден.

a)

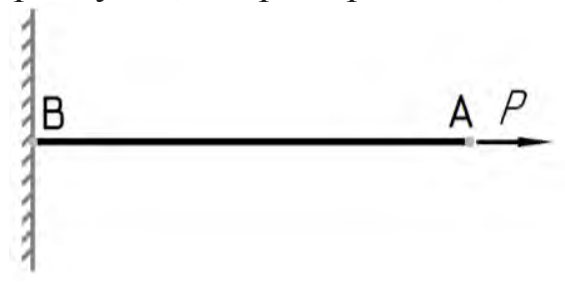

б)

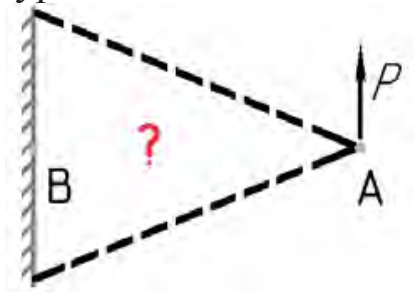

Рисунок 1 - Варианты передачи усилий в конструкции

Ещё сложнее принять решение, например, в трёхмерной задаче о выборе конструкции крыла малого удлинения. На рисунке 2 показаны два варианта решения по ориентации лонжеронов и стрингерного набора. В варианте а) прослеживается интуитивное стремление конструктора передавать воздушную нагрузку по кратчайшим путям, но при этом в длинных задних лонжеронах из-за малой строительной высоты будут возникать большие усилия в поясах. Не исключено, что схема б), которая организует передачу воздушной нагрузки через зоны крыла с большой строительной высотой, окажется более выгодной в весовом отноше- 
нии. Выбор оптимальной конструкции в этой задаче требует применения специальных методов [5].

Второй принцип, существенный для многих случаев нагружения, - эффективная работа одних и тех же элементов конструкции при различных вариантах нагружения. Второй принцип можно упрощённо сформулировать как обеспечение «взаимопомощи» элементов друг другу. Его хорошо иллюстрирует известная задача Разани [6]. Ферма, показанная на рисунке 3 , нагружается либо силой $P_{1}$, либо силой $P_{2}$.

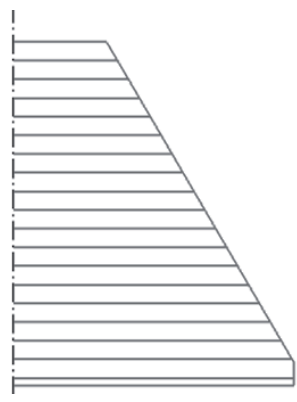

а) интуитивное решение

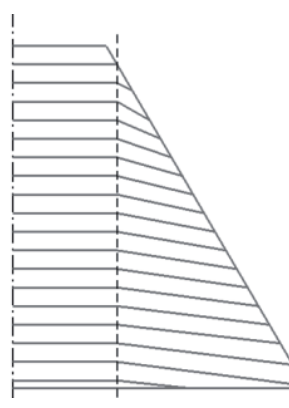

б) альтернативное решение

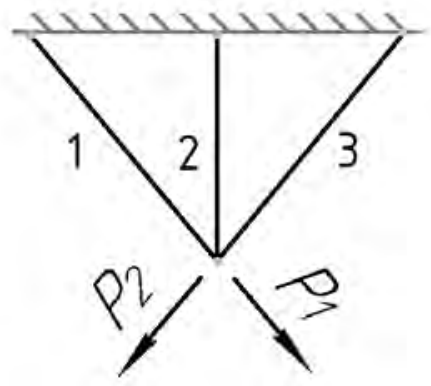

Рисунок 3 - Трёхстержневая ферма

Рисунок 2 - Крыло малого удлинения

Равнопрочная статически определимая ферма с двумя стержням 1 и 3 имеет массу боль-
чем статически неопределимая неравнопрочная ферма со стержнем 2, который работает в обоих случаях нагружения и частично разгружает стержни 1 и 3 в каждом из случаев нагружения. Решение подобных задач на практике также требует применения специальных методов оптимизации.

\section{2 Конструкции с минимальной потенциальной энергией деформации}

Для демонстрации некоторых теоретических идей и методов поиска идеальных конструкций, которые могут быть эффективными в сочетании с практически неограниченными возможностями аддитивных технологий, рассмотрим задачу о проектировании конструкции с минимальной потенциальной энергией деформации [7-10]. Будем считать, что конструкция выполнена из ограниченного по массе $m$ количества гипотетического материала с линейной зависимостью модуля упругости $E$ и допускаемого напряжения $[\sigma]$ от плотности $\rho$ :

$$
\begin{gathered}
E=\rho \cdot \bar{E}, \\
{[\sigma]=\bar{\sigma} \cdot \rho,}
\end{gathered}
$$

где $\bar{E}$ и $\bar{\sigma}$ - модуль упругости и допускаемое напряжение при единичной плотности. Назначим некоторое количество материала $m$ для создания 3D-модели, границы которой определяются заданными геометрическими ограничениями. Разобьём модель сеткой конечных твердотельных элементов. Предположим, что все исходные конечные элементы имеют одинаковые значения плотности и модуля упругости:

$$
\begin{gathered}
\rho_{0 i}=\frac{m}{V}, \\
E_{0 i}=\rho_{0 i} \cdot \bar{E},
\end{gathered}
$$

где индекс 0 - начальное распределение материала, $i$ - номер элемента, $V$ - общий объём всех конечных элементов. 
Рассматриваемую задачу оптимизации можно сформулировать как поиск конструкции с минимальной потенциальной энергией деформации при условии постоянства массы:

$$
U \rightarrow \min \text { при } m=\text { const }
$$

Плотности конечных элементов будем рассматривать в качестве проектных переменных:

$$
x_{i}=\rho_{i}, i=1 \ldots n,
$$

где $n$ - номер элемента в конечно-элементной модели. Проектные переменные $\rho_{i}$ ограничены условием сохранения массы конструкции:

$$
\sum_{i=1}^{n} \rho_{i} \cdot V_{i}=m
$$

где $V_{i}$ - объем і-го элемента.

Начальное (равномерное) распределение плотности материала в конструкции вычислим через объём конечных элементов:

$$
\rho_{0 i}=m \cdot\left(\sum_{i=1}^{n} V_{i}\right)^{-1}
$$

Рассчитаем напряженно-деформированное состояние (НДС) этой конструкции и вычислим эквивалентные напряжения в каждом элементе по удельной потенциальной энергии деформации:

$$
\sigma_{\text {eqv }}^{2}=\sigma_{1}^{2}+\sigma_{2}^{2}+\sigma_{3}^{2}-2 \mu \cdot\left(\sigma_{1} \cdot \sigma_{2}+\sigma_{2} \cdot \sigma_{3}+\sigma_{3} \cdot \sigma_{1}\right)
$$

Предполагая размеры элементов достаточно малыми, потенциальную энергию деформации 3D-модели запишем в следующем виде:

$$
U=\sum_{i=1}^{n} \frac{\sigma_{e q v_{0 i}}^{2}}{2 \cdot \rho_{i} \cdot \bar{E}} \cdot V_{i}
$$

При вычислениях по (10) предположим также, что напряжения в конечных элементах остаются неизменныли, в то время как плотности $\rho_{i}$ могут изменяться.

Воспользуемся методом множителей Лагранжа для поиска оптимальных значений плотностей элементов с учётом указанных допущений. Запишем следующую систему уравнений:

$$
\left\{\begin{array}{l}
\frac{\partial U}{\partial \rho_{i}}+\lambda \cdot \frac{\partial \varphi}{\partial \rho_{i}}=0,(i=1,2, \ldots, n) \\
\varphi\left(\rho_{i}\right)=\sum_{i=1}^{n} \rho_{i} \cdot V_{i}-m=0
\end{array}\right.
$$

где $\lambda$ - множитель Лагранжа, $\varphi$ - вспомогательная функция, отражающая постоянство массы (7).

С учётом (10) получим:

$$
\left\{\begin{array}{l}
-\frac{\sigma_{e q v_{0 i}}^{2} \cdot V_{i}}{2 \cdot \bar{E} \cdot \rho_{i}^{2}}+\lambda \cdot V_{i}=0,(i=1,2, \ldots, n) \\
\sum_{i=1}^{n} \rho_{i} \cdot V_{i}-m=0
\end{array}\right.
$$


Откуда

$$
\rho_{1 i}=\frac{\sigma_{e q v_{0 i}}}{\sqrt{2 \cdot \bar{E} \cdot \lambda}}
$$

Учитывая условие постоянства массы при изменении плотностей (7), новое распределение значений плотностей для каждого элемента запишем в следующем виде:

$$
\rho_{1 i}=\sigma_{e q v_{0 i}} \cdot \frac{m}{\sum_{i=1}^{n} \sigma_{e q v_{0 i}} \cdot V_{i}},
$$

где $\rho_{1 i}$ - новое распределение плотностей, используемое в дальнейшем в итерационном процессе вместо исходного $\rho_{0 i}$. Отметим простой физический смысл (14): новые плотности элементов назначаются пропорционально действующяи в них напряжениям.

Новым плотностям соответствует потенциальная энергия деформации $U_{0-1}$, определяемая напряжениями $\sigma_{e q v_{0 i}}$. Потенциальная энергия $U_{0-1}$ вычисляется путём подстановки плотностей $\rho_{1 i}$ в (10). Начальное значение потенциальной энергии $U_{0}$ конструкции определяется напряжениями $\sigma_{e q v_{0 i}}$ и плотностями элементов $\rho_{0 i}$. Новое значение потенциальной энергии $U_{0-1}$, исходя из смысла решения оптимизационной задачи (11), будет меньше, чем исходное:

$$
U_{0}>U_{0-1}
$$

При этом 3D-модель с распределением плотностей $\rho_{0 i}$ даёт распределение напряжений $\sigma_{0 i}$, которое соответствует условиям равновесия и совместности деформаций. В теле переменной плотности (ТПП) с другим распределением плотностей $\rho_{1 i}$ распределение напряжений $\sigma_{0 i}$ удовлетворяет только условиям равновесия, но не совместности деформаций.

Распределению плотностей элементов $\rho_{1 i}$ соответствует распределение напряжений $\sigma_{1 i}$ и потенциальная энергия $U_{1}$. В соответствии с принципом минимума энергии деформации:

$$
U_{0-1}>U_{1}
$$

Перерасчёт плотностей элементов по (14) и НДС, при котором новое распределение плотностей используется в качестве исходных данных, приводит к последовательности конструкций с потенциальными энергиями

$$
U_{0}>U_{0-1}>U_{1}>U_{1-2}>U_{2}>\ldots
$$

Эта монотонно убывающая последовательность ограничена снизу, поскольку энергия деформации величина положительная. Снижение значения потенциальной энергии до минимально возможного значения представляет собой поиск такого распределения плотностей, при котором конструкция обладает максимальной жёсткостью.

В процессе оптимизации плотности некоторых элементов могут обратиться в ноль, т.е. произойдёт вырождение элементов. При этом интенсивность деформации в невырожденных элементах становится одинаковой. Рассмотрим расчёт интенсивности деформаций в каждом элементе $i$ на $j+1$ итерации с учётом новых плотностей, определённых по (14):

$$
\varepsilon_{j+1 i}=\frac{\sigma_{e q v_{j+1 i}} \cdot \sum_{i=1}^{n} \sigma_{e q v_{j i}} \cdot V_{i}}{m \cdot \sigma_{e q v_{j i}} \cdot \bar{E}}
$$


Когда напряжения $\sigma_{e q v_{j+1 i}}$ становятся достаточно близкими к напряжениям $\sigma_{e q v_{j i}}$, процесс сходится, и получается следующее выражение, характеризующее интенсивность деформаций во всех невырожденных элементах:

$$
\varepsilon_{j+1 i}=\frac{\sum_{i=1}^{n} \sigma_{e q v_{j i}} \cdot V_{i}}{m \cdot \bar{E}} \approx \text { const }
$$

Основное расчётное соотношение итерационного алгоритма (14) при достижении сходимости, т.е. достаточно малых изменений напряжений и плотностей в каждом элементе, можно записать следующим образом, опустив номера соседних итераций:

$$
\frac{\sigma_{e q v_{i}}}{\rho_{i}}=\frac{\sum_{i=1}^{n} \sigma_{e q v_{i}} \cdot V_{i}}{m} \approx \text { const }
$$

Откуда следует, что найденное решение представляет собой равнонапряжённое в смысле гипотезы (2) ТПП.

ТПП с минимальной энергией деформации обладает ещё одним интересным и полезным для практических приложений свойством. Выберем из последовательности (17) «промежуточные» значения энергии

$$
U_{0-1}>U_{1-2}>\ldots,
$$

которые также образуют сходящуюся последовательность. Запишем выражения для этих величин, используя подстановку (14) в (10):

$$
\frac{1}{2 \cdot \bar{E} \cdot m} \cdot\left(\sum_{i=1}^{n} \sigma_{e q v_{0 i}} \cdot V_{i}\right)^{2}>\frac{1}{2 \cdot \bar{E} \cdot m} \cdot\left(\sum_{i=1}^{n} \sigma_{e q v_{1 i}} \cdot V_{i}\right)^{2}>\ldots
$$

Обозначим переменную величину $\sum_{i=1}^{n} \sigma_{e q v_{j i}} \cdot V_{i}$ в последовательности (22) символом $G_{j}$, где индекс обозначает номер итерации, и будем называть её силовым фактором, следуя [11] - «load carring factor» (LCF).

B процессе оптимизации эти величины также образуют сходящуюся последовательность $\mathrm{G}_{0}>\mathrm{G}_{1}>\ldots$. достигают минимума в конструкции с минимальной энергией деформации.

Если не использовать конечно-элементных представлений, то величину силового фактора можно определить в общем виде как интеграл от эквивалентных напряжений по объёму конструкции:

$$
G=\int_{V} \sigma_{e q v} \cdot d V
$$

Силовой фактор учитывает одновременно величину внутренних усилий в конструкции и протяжённость их действия.

В простейшем случае стержневой конструкции (фермы) силовой фактор - это сумма модулей усилий $N$ в стержнях, умноженных на их длину $l$

$$
G_{\text {truss }}=\sum_{i=1}^{n}\left|N_{i}\right| \cdot l_{i}
$$

Через силовой фактор и допускаемое напряжение [ $\sigma$ ] может быть вычислен теоретически необходимый объём $\mathrm{V}_{\text {т }}$ стержневой или тонкостенной конструкции из традиционного материала или теоретически необходимая масса $m_{\text {т }}$ конструкции из материала переменной плотности со свойством (2) 


$$
\begin{gathered}
V_{T}=\frac{G}{[\sigma]} \\
m_{T}=\frac{G}{\bar{\sigma}}
\end{gathered}
$$

Чем меньше силовой фактор, тем конструкция легче. Вычислительные эксперименты показывают, что силовой фактор стержневых и тонкостенных безмоментных конструкций определяется в основном силовой схемой и относительно слабо зависит от распределения материала. Силовой фактор трёхмерных конструкций из материала с переменной плотностью сильно зависит от распределения плотностей и может уменьшаться на десятки процентов в процессе оптимизации. Интегральная величина - силовой фактор - позволяет построить эффективные, так называемые «весовые формулы», для прогнозирования и сравнения массы конструкций на ранних стадиях проектирования [12].

\section{3 Топологическая оптимизация пространственных конструкций}

На основе рассмотренного решения задачи о минимизации потенциальной энергии конструкции переменной плотности может быть предложен следующий эвристический алгоритм топологической оптимизации с учётом ограничений по прочности при многих случаях нагружения.

1) В геометрические ограничения конструкции вписывается непрерывная упругая среда, разбиваемая сеткой конечных элементов типа “solid".

2) Задаются начальное распределение материала во всех элементах конструкции и минимально допустимая величина плотности элементов $\rho_{\min }$. Удобно использовать $\rho_{0 i}=$ const $>\rho_{\min }$, здесь индекс 0 - номер итерации. $\rho_{\min }$ может быть достаточно малой величиной, необходимой только для вычисления НДС ТПП.

3) Вычисляется масса конструкции

$$
m_{0}=\sum_{i=1}^{n} \rho_{0 i} \cdot V_{i}
$$

4) Назначаются механические характеристики элементов.

5) Вычисляются по (1) и (2) удельные характеристики прочности $\bar{\sigma}$ и упругости $\bar{E}$ предполагаемого к использованию конструкционного материала.

6) Выполняется расчёт НДС трёхмерного тела на заданные внешние нагрузки.

7) Вычисляются эквивалентные напряжения по определенной теории прочности, например по Мизесу, в каждом элементе для каждого случая нагружения

$$
\sigma_{\text {eqv }}^{2}=\sqrt{\sigma_{1}^{2}+\sigma_{2}^{2}+\sigma_{3}^{2}-\left(\sigma_{1} \cdot \sigma_{2}+\sigma_{2} \cdot \sigma_{3}+\sigma_{3} \cdot \sigma_{1}\right)}
$$

и выбираются максимальные $\sigma_{e q v_{\max } j}$, где $j$ - номер итерации, $i$ - номер элемента.

8) Вычисляется интегральная характеристика - силовой фактор G

$$
G_{j}=\sum_{i=1}^{n} \sigma_{e q v_{\max j i}} \cdot V_{i}
$$

9) Определяются избытки прочности каждого элемента

$$
\eta_{j i}=\frac{\rho_{j i} \cdot \bar{\sigma}}{\sigma_{e q v_{\max } j i}}
$$


Значения $\eta_{j i}<1$ означают недостаточную прочность $i$-го элемента.

10) Выбирается минимальное значение $\eta_{j \min }$ по всем элементам, и пропорциональным изменением плотностей всех элементов определяется потребная по прочности масса конструкции:

$$
m_{j}^{*}=\frac{m_{j}}{\eta_{j \min }}
$$

11) Вычисляются новые плотности элементов

$$
\rho_{j+1 i}=\frac{\sigma_{e q v_{\max } j i}}{\bar{\sigma}}
$$

12) Если $\rho_{j+1 i}<\rho_{\min _{i}}$, то принимается $\rho_{j+1 i}=\rho_{\min _{i}}$

13) Вычисляется масса конструкции $m_{1}$ с плотностями элементов $\rho_{1 i}\left(\rho_{j+1 i}\right.$ и масса $m_{j}$ в итерационном процессе):

$$
m_{1}=\sum_{i=1}^{n} \rho_{1 i} \cdot V_{i}
$$

Эту величину можно назвать массой «условно равнопрочной конструкции», так как назначение новых плотностей по (31) вызывает изменение НДС конструкции и отклонение $\eta_{\text {j min }}$ от единицы.

14) Вычисляются новые модули упругости и допускаемые напряжения элементов

$$
\begin{gathered}
E_{j+1 i}=\rho_{j+1 i} \cdot \bar{E} \\
{[\sigma]_{j+1 i}=\rho_{j+1 i} \cdot \bar{\sigma}}
\end{gathered}
$$

Далее весь расчёт повторяется с п. 6 до стабилизации. Для контроля целесообразно использовать условие $1-\eta_{\min } \leq \varepsilon$, где $\varepsilon$ - малая заданная величина, например 0,01 в инженерных расчётах. Сходимость данного процесса наблюдается через 10-30 итераций в зависимости от особенностей задач.

В завершение оптимизации анализируется распределение материала и главных напряжений внутри заданных геометрических ограничений проектируемой конструкции. По результатам анализа предлагается несколько вариантов конструкций, которые могут быть изготовлены традиционными способами или с использованием аддитивных технологий. Сравнение силового и весового совершенства вариантов целесообразно делать с использованием критериев (29), (30), (31) и (33).

Приведенный алгоритм описан с подробностью, которая позволяет использовать его для реализации новой проектной парадигмы concurrent design - «технология точного попадания», описанной в [9]. Обращаем внимание на использование в нём новых для конструктора понятий «силовой фактор», «условно равнопрочная конструкция» и включение в проектный итерационный процесс известного понятия «избыток прочности» из традиционных поверочных расчётов в технологии эволюционного проектирования. Все эти три понятия имеют чёткое математическое описание. Наблюдение за изменениями указанных критериев в итерационном процессе обладает, по нашему мнению, большим обучающим потенциалом. 


\section{4 Примеры}

\section{1 Проектирование балки}

В качестве простого примера проектирования и изготовления аддитивной конструкции рассматривалась балка, предназначенная для испытаний на трёхточечный изгиб. Геометрические ограничения балки, условия опирания, нагрузка и схема разбиения на элементы даны на рисунке 4. Здесь же показано распределение плотности материала на второй итерации. Оптимальное по критерию равнопрочности распределение плотности материала в ТПП показано на рисунке 5.

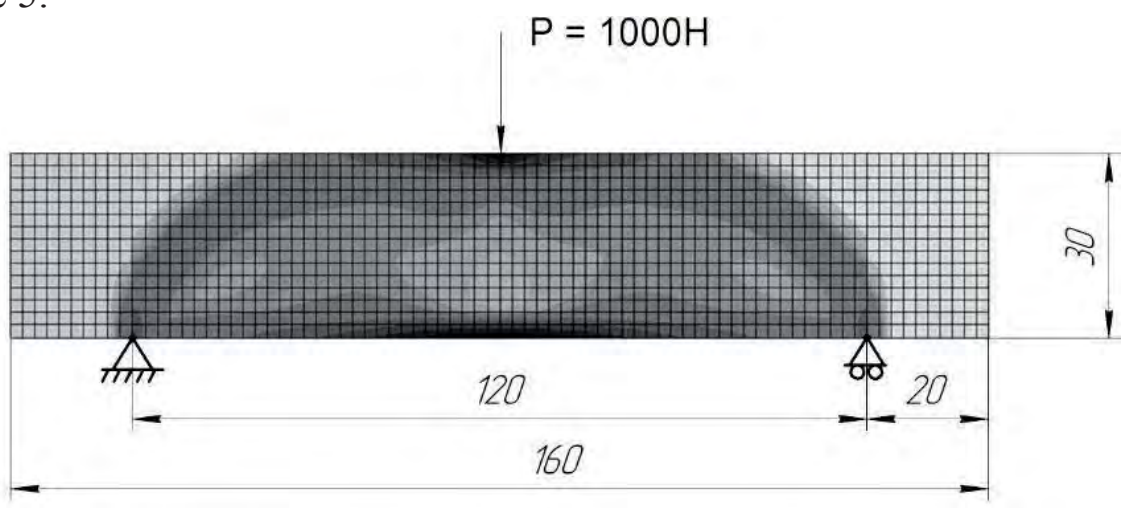

Рисунок 4 - Плоская проектная задача

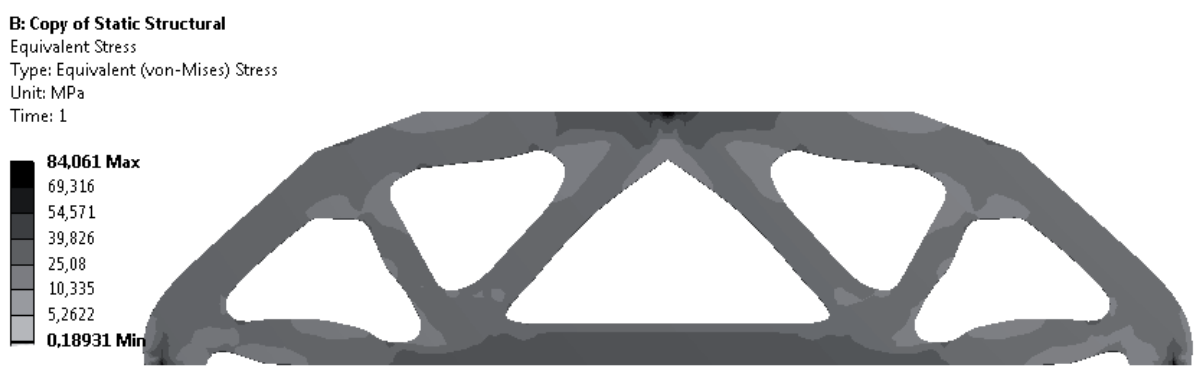

Рисунок 5 - Распределение плотностей в элементах конструкции

Структура конструкции, показанная на рисунке 6, может быть выбрана по этой информации как рациональная. Силовой фактор этой фермы имеет величину $G_{\text {truss }}=0,221 \times 10^{6} \mathrm{H}$. мм, что превышает показатель равнопрочного ТПП $\mathrm{G}_{\text {тпп }}=0,201 \times 10^{6}$ на величину $\Delta=9 \%$.

На рисунке 6 показано испытание спроектированной и изготовленной аддитивной конструкции на трёхточечный изгиб до разрушения.

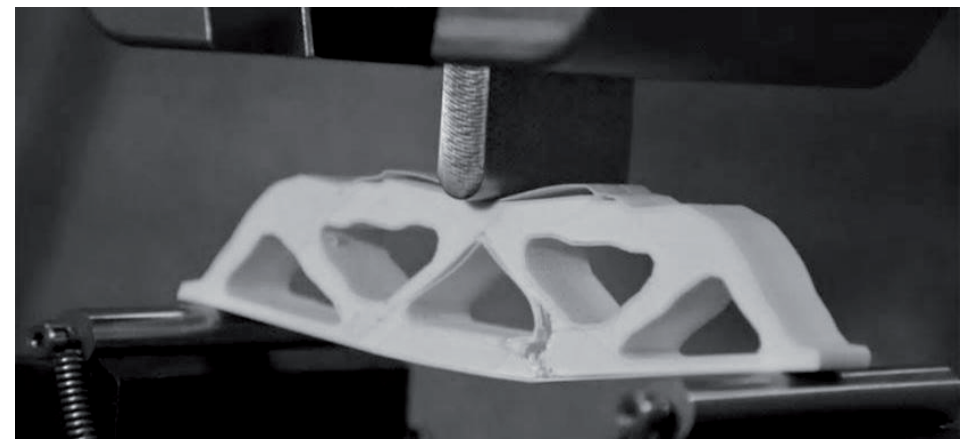

Рисунок 6 - Испытание рациональной аддитивной конструкции 
Одновременно с фермой в едином технологическом процессе изготавливались стандартные образцы для снятия механических характеристик материала. Расчёт по методу конечных элементов разрушающего усилия для фермы по этим характеристикам дал удовлетворительное совпадение результатов.

Рассмотренная топологическая оптимизация плоских конструкций в настоящее время достаточно проработана для использования на практике. Другие возможные модели ТПП, отличные от (1) и (2), и интересные примеры решений можно найти в $[13,14]$.

Замечания о терминах.

В рассмотренном примере для описания эффективности проектов на вербальном уровне использованы термины «оптимальная» и «рациональная» конструкции. На наш взгляд, термин «оптимальная» уместно использовать когда чётко определены: целевая функция, проектные переменные и ограничения. Термин «рациональная» используется в современной технической литературе широко и не однозначно. Довольно часто он используется при принятии проектных решений с учётом многих факторов и многих параметров, которые хотелось бы получить в виде max или min. То есть речь идёт о компромиссах или множестве Парето, даже если оно и не упоминается ни в какой форме. В ряде случаев «рациональным» решением объявляется выбор из двух-трёх возможных вариантов. Поэтому в каждом конкретном тексте этот термин требует пояснений. Лучше всего, когда есть оценка предела совершенства, полученная из упрощённой или идеализированной задачи. Тогда о степени «рациональности» технического решения можно судить по "penalty» - уступке или недобору эффективности проекта по сравнению с идеалом. Например, коэффициент полезного действия тепловой машины определяет её эффективность по сравнению с идеальным циклом Карно. В нашем примере - это отношение реальной массы конструкции к теоретической, вычисляемой через силовой фактор.

\section{2 Проектирование пространственной конструкции}

Рассмотрим пример проектирования пространственной конструкции для передачи крутящего момента $\mathrm{M}=10^{8} \mathrm{H} \cdot$ мм внутри прямоугольного параллелепипеда ABCDEFGH, с размерами $\mathrm{AB}=100, \mathrm{AD}=500, \mathrm{AE}=1000$ (см. рисунок 7).

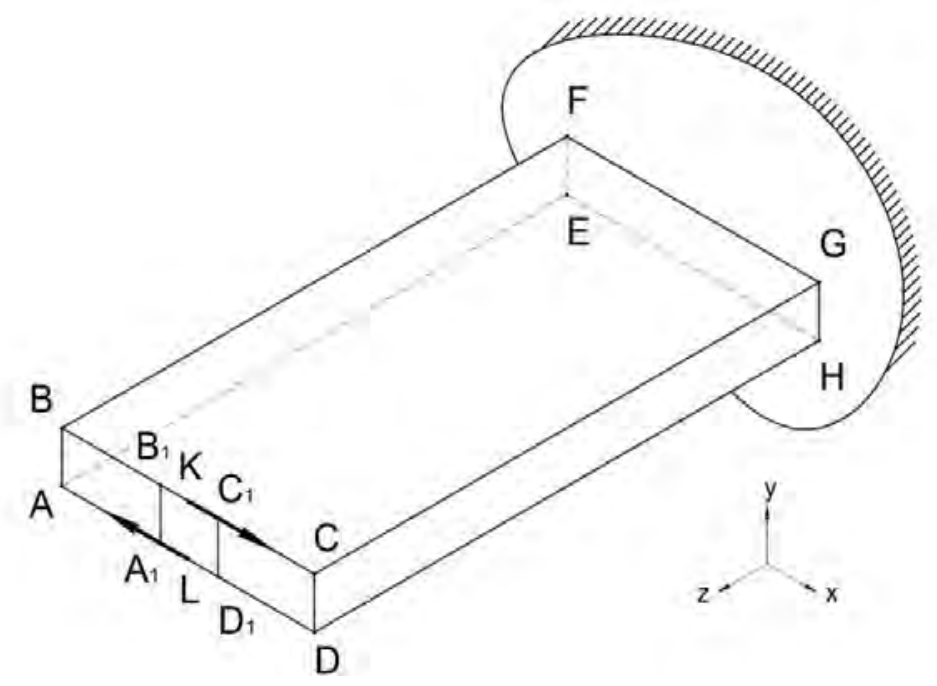

Рисунок 7 - Пространственная проектная задача

Нагрузка считается приложенной в виде пары сил в точках K и L. Закрепление конструкции возможно на площадке EFGH. Эта задача моделирует с определёнными упрощениями проектирование неподвижной части крыла самолёта с изменяемой стреловидностью. На ри- 
сунке 8 штриховкой выделена та часть крыла, в которой можно разместить силовую конструкцию. Вопрос в том, какой она должна быть?

В качестве простых решений задачи могут быть предложены интуитивно два варианта конструкций: а) круговая замкнутая цилиндрическая оболочка с поперечным сечением, вписанным в квадрат $\mathrm{A}_{1} \mathrm{~B}_{1} \mathrm{C}_{1} \mathrm{D}_{1}$ и б) цилиндрическая оболочка с тем же прямоугольным поперечным сечением.

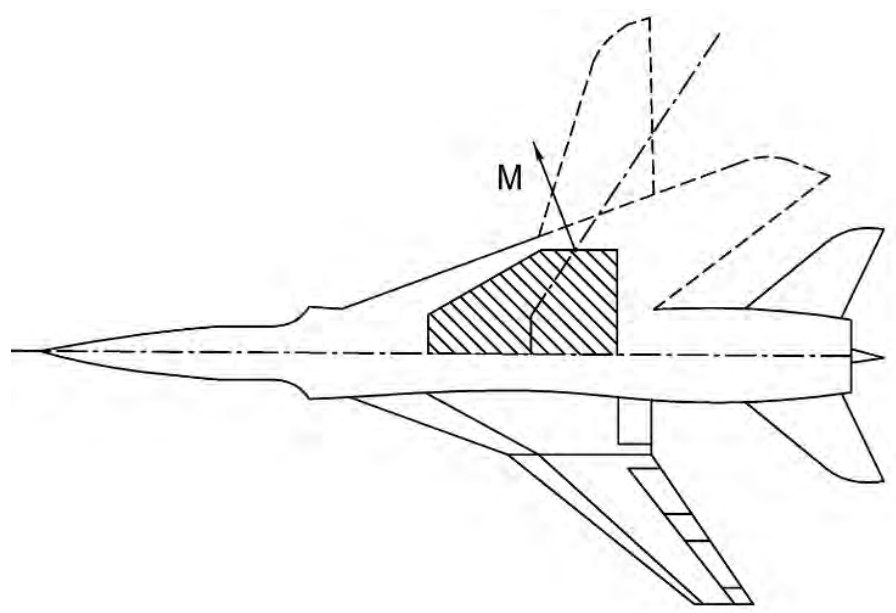

Рисунок 8 - Фрагмент силовой конструкции крыла с изменяемой стреловидностью

При расчёте касательных напряжений в этих оболочках по формуле Бредта и эквивалентных напряжений по Мизесу $\sigma_{\text {eqv }}=\tau \sqrt{3}$ получается одинаковая (!) величина силового фактора оболочек $G=3,46 \cdot 10^{9} \mathrm{H} \cdot$ мм для вариантов (а) и (б). Крутящий момент М в виде пары сил $\mathrm{P}=\mathrm{M} / \mathrm{KL}$ может быть передан на круговую оболочку с помощью кольца диаметром $\mathrm{d}=\mathrm{KL}$, материал которого работает с переменными усилиями в одноосном состоянии. Дополнительный силовой фактор кольца в задаче имеет величину $\Delta G=\frac{1}{4} M \cdot \pi=0,079 \cdot 10^{9} \mathrm{H} \cdot$ мм. В итоге силовой фактор конструкции в виде цилиндрической оболочки с кольцом (шпангоутом) на свободном торце составляет $G_{\text {круг }}=3,54 \cdot 10^{9} \mathrm{H} \cdot$ мм.

Соответственно крутящий момент в виде пары сил может быть передан на квадратную тонкостенную оболочку с помощью торцевой стенки и пары стержней $\mathrm{B}_{1} \mathrm{C}_{1}$ и $\mathrm{A}_{1} \mathrm{D}_{1} \mathrm{c}$ дополнительным силовым фактором $\Delta G=0,137 \cdot 10^{9} \mathrm{H} \cdot$ мм. Силовой фактор квадратной конструкции с зашивкой на свободном торце составляет $G_{\kappa в}=3,60 \cdot 10^{9} \mathrm{H} \cdot$ мм.

Таким образом, при одинаковых габаритах конструкция с квадратным сечением неожиданно оказывается несколько менее эффективной, чем круговая, несмотря на большую «световую площадь». Разница составляет менее $2 \%$, и поэтому имеет только теоретический интеpec.

Аналогичные расчёты тонкостенных конструкций с прямоугольными поперечными сечениями с соотношениями «ширины к высоте» $\mathrm{B}_{1} \mathrm{C}_{1} / \mathrm{CD}=2 / 1$ и $\mathrm{BC} / \mathrm{CD}=5 / 1$ дают величины силового фактора конструкций соответственно $G_{2 / 1}=2,79 \cdot 10^{9}$ и $G_{5 / 1}=2,41 \cdot 10^{9} \mathrm{H} \cdot$ мм.

Эти результаты также представляются несколько неожиданными - увеличение «световой площади» прямоугольных поперечных сечений в 2 и 5 раз даёт уменьшение силового фактора по сравнению с круговой и квадратной конструкцией только на 22,5\% и 33\% соответственно. В подобных ситуациях перебора вариантов конструкций представляет интерес знание предела совершенства - идеального решения. 
С этой целью рассмотрим решение обсуждаемой модельной задачи с использованием алгоритма топологической оптимизации (раздел 3, пункты алгоритма 1-14). В качестве исходных данных использовались следующие характеристики конструкционного материала: плотность $\rho=2,8$ г $/ \mathrm{cm}^{3}$, модуль упругости $\mathrm{E}=70000$ МПа, коэффициент Пуассона $\mu=0,3$, допускаемое напряжение $[\sigma]=300$ МПа. Эквивалентные напряжения вычислялись по критерию Мизеса. Расчёты НДС выполнялись в системе ANSYS с использованием кубических элементов Solid-65 с размером ребра 0,1CD. Узлы на плоскости EFGH считались закреплёнными по трём степеням свободы.

На рисунке 9 показано равнопрочное в смысле (20) распределение материала в конструкции. Эти картины отчётливо показывают, как крутящий момент в виде пары сил на первых $10 \%$ оси Z конструкции трансформируется через массивную часть в полую конструкцию переменного (увеличивающегося) сечения. Начиная примерно с $80 \%$ оси Z, передача крутящего момента в оптимальной конструкции постепенно перестраивается в бимоментную структуру у заделки.

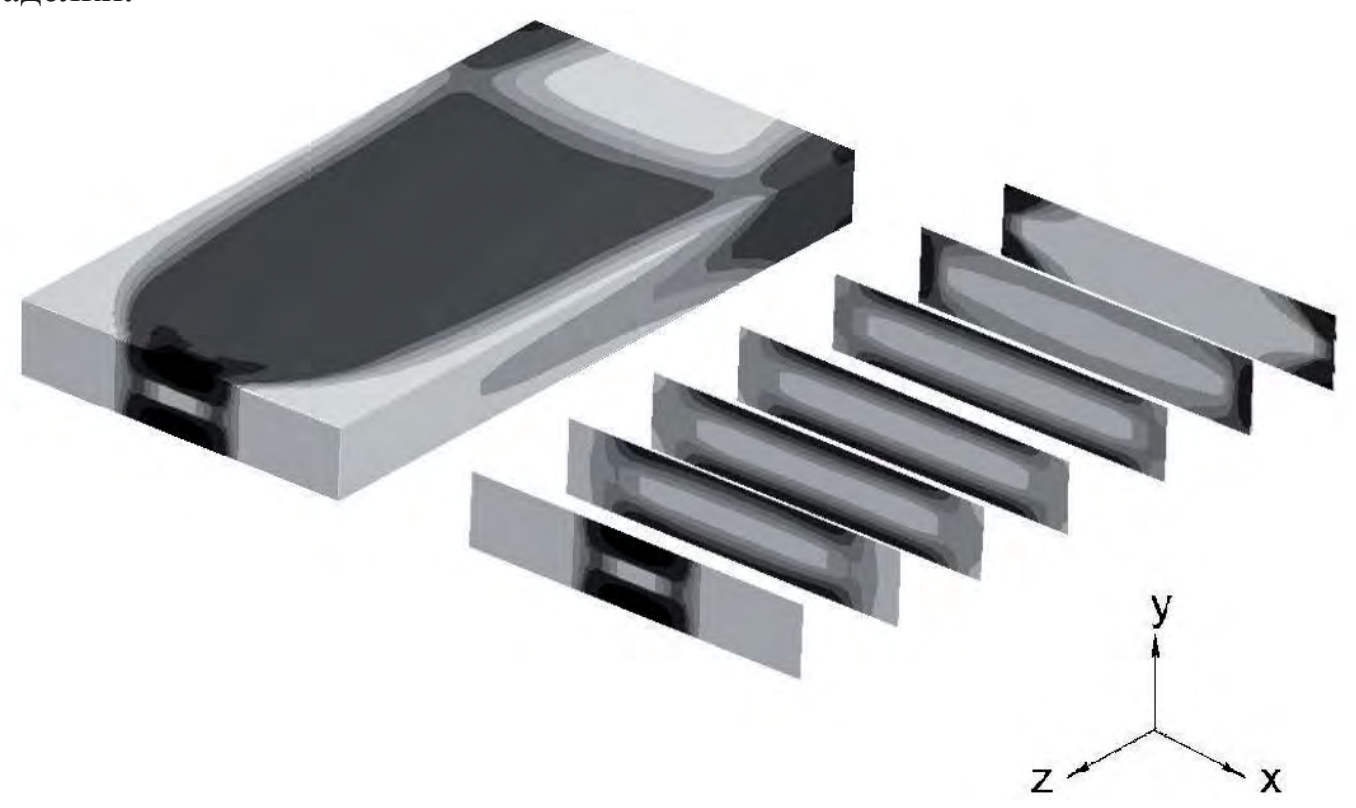

Рисунок 9 - Оптимальное распределение материала

В качестве начального распределения плотностей принималась постоянная величина во всех элементах. На рисунках 10, 11, и 12 показано по итерациям $j$ : изменение силового фактора G; массы условно-равнопрочной конструкции $m_{j}$ и массы потребной по прочности $m_{j}^{*}$; минимального коэффициента избытка прочности $\eta_{j m i n}$.

Потребность в довольно большом числе итераций объясняется «сильной» статической неопределимостью ТПП, представленного набором элементов. Изменение плотности и связанного с ней модуля упругости каждого элемента вызывает изменение распределения напряжений во всех остальных.

Величина силового фактора в полученной идеальной конструкции меньше, чем во всех рассмотренных интуитивно-рациональных конструкциях, таблица 1. Это означает её превосходство по способу передачи усилий.

Таблица 1 - Величины силового фактора

\begin{tabular}{|c|c|c|c|c|}
\hline$G_{\text {квадрат, }}, \mathrm{H} \cdot$ мм & $G_{\text {круг }}, \mathrm{H} \cdot$ мм & $G_{\text {прямуг.2 } / 1}, \mathrm{H} \cdot$ мм & $G_{\text {прямоуг.5 } / 1, \mathrm{H} \cdot \mathrm{MM}}$ & $G_{\text {opt }}, \mathrm{H} \cdot \mathrm{MM}$ \\
\hline $3,60 \cdot 10^{9}$ & $3,54 \cdot 10^{9}$ & $2,79 \cdot 10^{9}$ & $2,41 \cdot 10^{9}$ & $2,29 \cdot 10^{9}$ \\
\hline
\end{tabular}




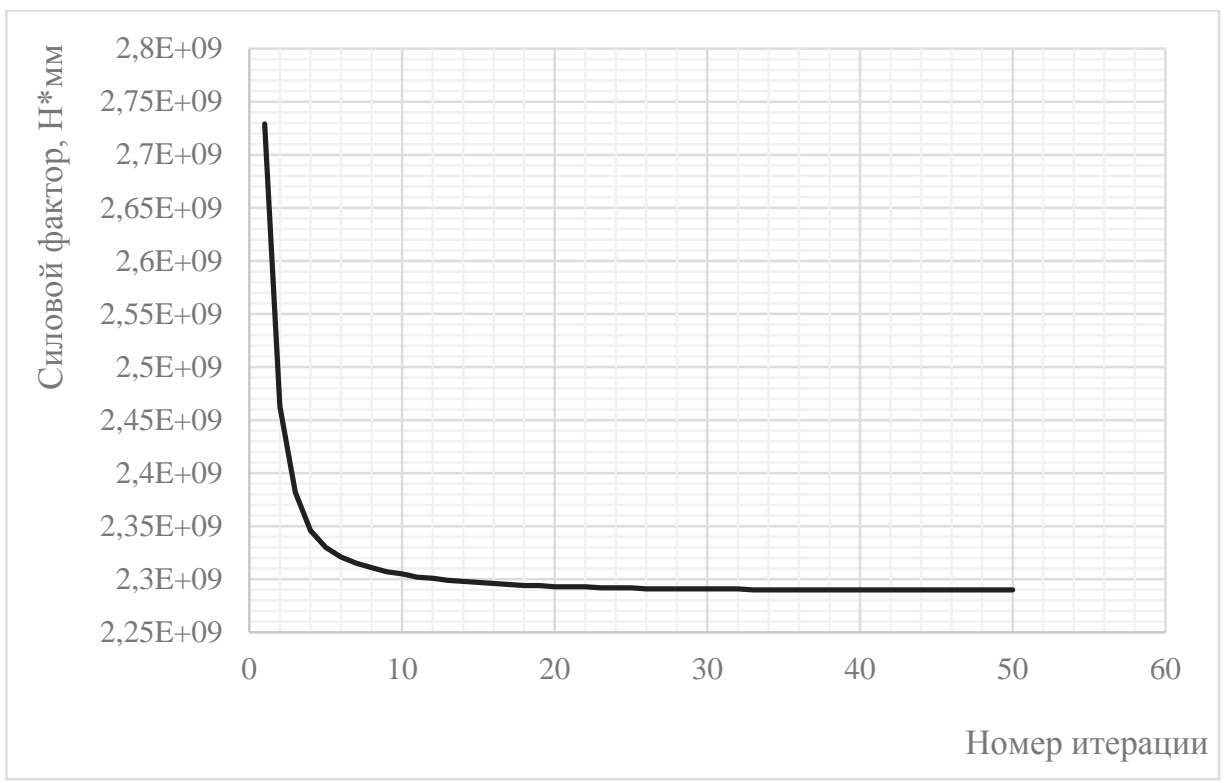

Рисунок 11 - Изменение силового фактора в процессе оптимизации

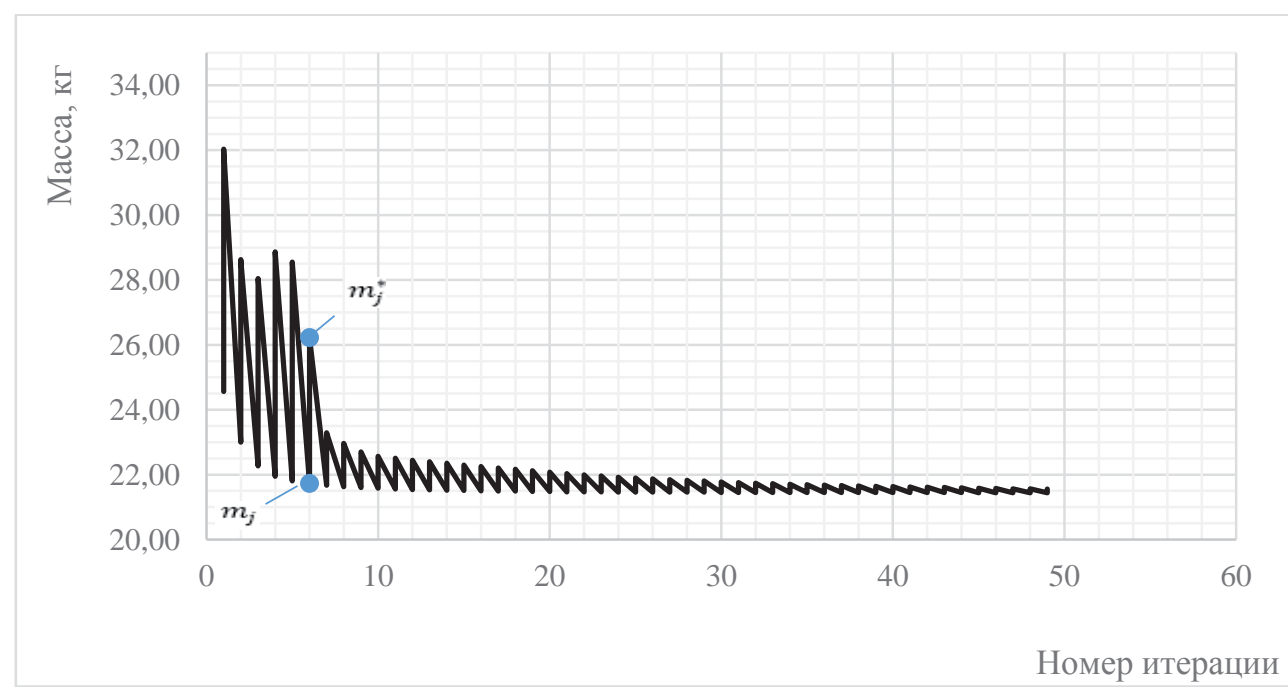

Рисунок 12 - Изменение масс в процессе оптимизации

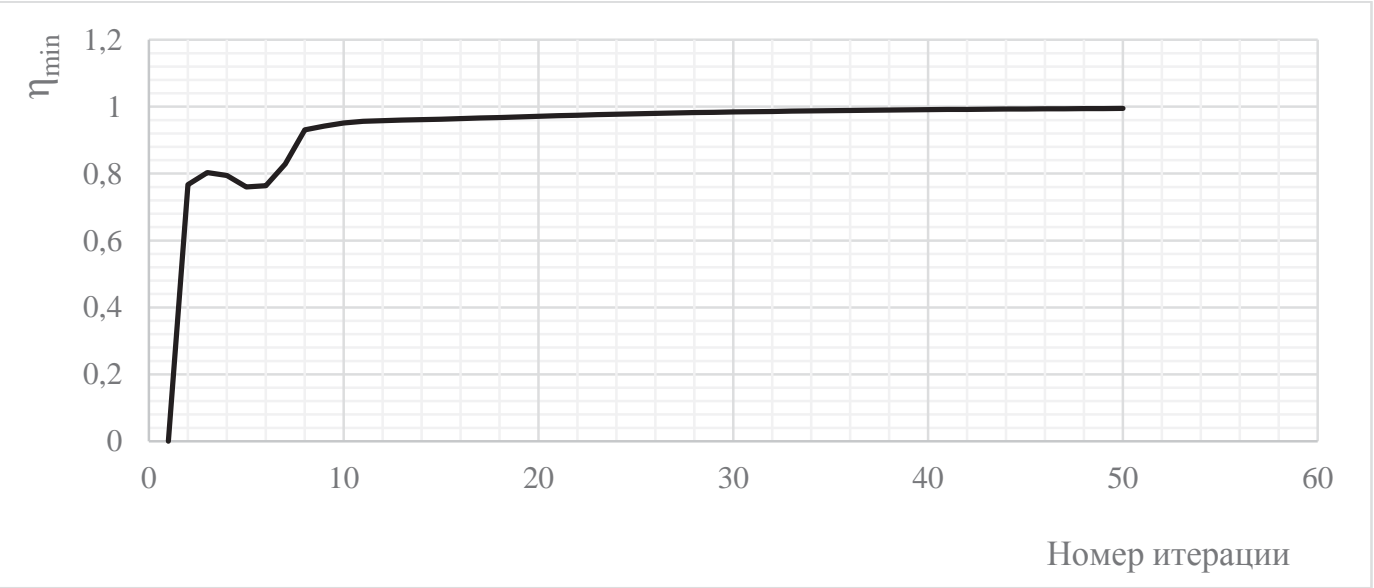

Рисунок 13 - Изменение минимального коэффициента избытка прочности в процессе оптимизации 


\section{Заключение}

Рассмотренный алгоритм проектирования и примеры показывают, что оптимизационная модель в виде ТПП позволяет решать две самостоятельные задачи. Во-первых, получать количественную оценку предела совершенства конструкции с точки зрения минимизации её массы. Во-вторых, показать её рациональную силовую схему.

Пример с трёхмерной конструкцией показывает насколько перспективна разработка аддитивных технологий, которые позволяли бы создавать изотропные и анизотропные материалы переменной плотности в широком диапазоне её значений. На микроуровне это могли бы быть рациональные стержневые или ячеистые структуры с возможностями задавать поперечные сечения стержней и толщины ячеек. Заметим, что примерно так устроены такие совершенные конструкции, как кости животных, особенно птиц.

\section{Благодарности}

Работа выполнена при поддержке Министерства науки и образования Российской Федерации в рамках реализации программы повышения конкурентоспособности Самарского университета среди ведущих образовательных и научных центров на 2013-2020 годы.

Автор благодарит А.А. Павлова и С.А. Павлову за подготовку иллюстративных материалов.

\section{Список источников}

[1] Лычев, С.А. Моделирование аддитивных технологий: геометрический подход / С.А. Лычев, А.В. Манжиров, Е.В. Мурашкин // Сборник докладов II Международной конференции «Аддитивные технологии: настоящее и будущее» Москва, 16 марта 2016 г., ВИАМ. - 2016. - С.18.

[2] Промахов, В.B. О возможности получения аддитивных керамических структур с использованием метода послойного наплавления. / В.В. Промахов, И.А. Жуков, С.А. Ворожцов, М.В. Шевченко, Б.Г. Третьяков, А.С. Жуков, А.Б. Ворожцов // Сборник докладов II Международной конференции «Аддитивные технологии: настоящее и будущее» Москва, 16 марта 2016 г., ВИАМ. - 2016. - С.12.

[3] Tapacoвa, T.B. Возможности технологий аддитивного производства для изготовления машиностроительной продукции различного назначения / Т.В. Тарасова, А.А. Филатова // Сборник докладов II Международной конференции «Аддитивные технологии: настоящее и будущее» Москва, 16 марта 2016 г., ВИАМ. 2016. - С.32.

[4] Горбовеи, М.A. Усталостные свойства жаропрочных материалов, полученных методом SLM. / M.A. Горбовец, А.Г. Евгенов, И.А. Ходинев, М.И. Лукьянова // Сборник докладов II Международной конференции «Аддитивные технологии: настоящее и будущее» Москва, 16 марта 2016 г., ВИАМ. - 2016. - С.8.

[5] Машиностроение. Энциклопедия. Том IV-21. Самолеты и вертолеты. Книга 2. Проектирование, конструкции и системы самолетов и вертолетов. Под общей редакцией А.М. Матвеенко - М.: Машиностроение, 2004. - 592 c.

[6] Разани, P. Поведение равнопрочной конструкции и её отношение к конструкции минимального веса / Р. Разани // Ракетная техника и космонавтика. - 1965. - Том 3. - №12. - С.35-39.

[7] Комаров, А.А. Основы проектирования силовых конструкций / А.А. Комаров. - Куйбышевское книжное издательство, 1965. - 88 с.

[8] Комаров, В.A. Проектирование силовых схем авиационных конструкций / В.А. Комаров // Актуальные проблемы авиационной науки и техники. - М.: Машиностроение, 1984. - с. 114-129.

[9] Комаров, В.A. Точное проектирование / В.А. Комаров // Онтология проектирования. №3(5), 2012. - с.8-23.

[10] Komarov, V.A. Aircraft design using variable density model / V.A. Komarov, A.V. Boldyrev, A.S. Kuznetsov, M.Yu. Lapteva // Aircraft Engineering and Aerospace Technology, Vo. 84, No. 3, 2012, pp. 162-171.

[11] Vasiliev V.V. Optimal Design: Theory and Applications to Materials and Structures / V.V. Vasiliev, Z. Gurdal // CRC Press, 1999. - 320 p.

[12] Комаров, В.А. Весовой анализ авиационных конструкций: теоретические основы / В.А. Комаров // Общероссийский научно-технический журнал «Полет», 2000 (№1). - С.31-39. 
[13] Bendsoe, M.P. Topology Optimization: Theory, Methods and Applications / M.P. Bendsoe, O. Sigmund. - New York: Springer, 2003.- $271 \mathrm{p}$

[14] Bendsoe, M.P. Material interpolation schemes in topology optimization / M.P.Bendsoe, O. Sigmund // Archive of Applied Mechanics. - 1999. - Volume 69, Issue 9-10, p. 635-654.

\title{
THEORETICAL BASIS FOR DESIGN OF LOAD-BEARING STRUCTURES PRODUCED USING ADDITIVE TECHNOLOGIES
}

\author{
V.A. Komarov \\ Samara National Research University named after academician S.P. Korolev, Samara, Russia \\ vkomarov@ssau.ru
}

\begin{abstract}
The article discusses interconnections and mutual influence between the triad of the basic engineering concepts - material, technology and structure. The possibilities for development of load-bearing structures, produced using additive technologies are analyzed. The task of optimal material distribution within a body of variable density is formulated and solved. An algorithm for topologic optimization of load-bearing structures, produced form a material of variable density, based on the criteria of minimal mass is proposed. General mechanical properties of the hypothetical material are assumed to be proportional to its density. The limitations of strength and rigidity are considered for single and multiple loading cases. The content of the concepts "optimal" and "rational" structure is discussed and refined. The "force factor" objective criterion, which takes into account the magnitude and extent of the action of internal forces, is proposed as a measure of comparison of the weight perfection of various designs and to estimate the design proximity to a theoretically optimal solution. The article presents examples of two and three dimensional structures. Future goals are formulated to develop new materials and methods for creating structures with variable density as one of the possible directions for the further development of additive technologies.
\end{abstract}

Key words: additive technologies, design, structure, strength, stiffness, material, optimization, the body of variable density.

Citation: Komarov VA. Theoretical basis for design of load-bearing structures produced using additive technologies. Ontology of designing, 2017; 7(2): 191-206. DOI: 10.18287/2223-9537-2017-7-2-191-206.

\section{Acknowledgment}

The work was carried out with the support of the Russian Ministry of Science and Education under the realization of the programme of increase of the competitiveness of the Samara University amongst the leading educational and scientific centers for 2013-2020. The author thanks AA. Pavlova and S.A. Pavlov for preparing illustrative materials.

\section{References}

[1] Lychev SA, Manzhirov AV, Murashkin EV. Modeling of additive technologies: geometric approach [In Russian]. Proceedings of the II International Conference "Additive Technologies: Present and Future" Moscow, March 16, 2016, VIAM. 2016. - p.18

[2] Promakhov VV, Zhukov IA, Vorozhtsov SA, Shevchenko MV, Tretyakov BG, Zhukov AS, Vorozhtsov AB. On the possibility of obtaining additive ceramic structures using the layer-by-layer melting technique [In Russian]. Proceedings of the II International Conference "Additive Technologies: Present and Future" Moscow, March 16, 2016, VIAM. 2016. - p.12

[3] Tarasova TV, Filatova AA Possibilities of additive production technologies for the manufacture of machinebuilding products for various purposes [In Russian]. Proceedings of the II International Conference "Additive Technologies: Present and Future" Moscow, March 16, 2016, VIAM. 2016. - p.32.

[4] Gorbovets MA, Evgenov AG, Khodinev IA, Lukyanova MI. Fatigue properties of high-temperature materials obtained by the SLM method [In Russian]. Proceedings of the II International Conference "Additive Technologies: Present and Future" Moscow, March 16, 2016, VIAM. 2016. - p.8. 
[5] Mechanical engineering. Encyclopedia. Volume IV-21. Aircraft and helicopters. Book 2. Design, construction and systems of aircraft and helicopters. General edition A.M. Matveenko - Moscow: Mechanical Engineering, 2004. 592 p.

[6] Razani R. Behavior of Fully-stressed Structures and Its Relation to the Design of Minimal Weight structure [In Russian]. Rocket Technology and Astronautics. - 1965. - Volume 3. - № 12. - p.35-39.

[7] Komarov AA. Fundamentals of load-bearing structures design [In Russian]. Kuibyshev book publishing house, 1965. - 88 p.

[8] Komarov VA. Design of aircraft load-bearing structures [In Russian]. Actual problems of aviation science and technology. - M.: Mechanical Engineering, 1984. - p. 114-129.

[9] Komarov VA. Concurrent Design [In Russian]. Ontology of Designing. 2012; 3(5): 8-23.

[10] Komarov VA, Boldyrev AV, Kuznetsov AS, Lapteva MYu. Aircraft design using variable density model, Aircraft Engineering and Aerospace Technology, Vo. 84, No. 3, 2012, pp. 162-171.

[11] Vasiliev VV, Gurdal Z. Optimal Design: Theory and Applications to Materials and Structures. CRC Press, 1999. $320 \mathrm{p}$.

[12] Komarov VA. Weight Analysis of Aviation Structures: Theoretical Foundations [In Russian]. All-Russian Scientific and Technical Journal "Polet", 2000 (№1). - p.31-39.

[13] Bendsoe MP, Sigmund O. Topology Optimization: Theory, Methods and Applications. - New York: Springer, 2003.- $271 \mathrm{p}$

[14] Bendsoe MP, Sigmund O. Material interpolation schemes in topology optimization. Archive of Applied Mechanics. - 1999. - Volume 69, Issue 9-10, p. 635-654.

\section{Сведения об авторе}

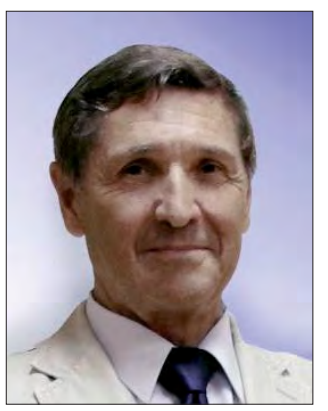

Комаров Валерий Андреевич, 1941 г. рождения. Окончил Куйбышевский авиационный институт в 1964 году, доктор технических наук (1976), профессор (1978), заведующий кафедрой конструкции и проектирования летательных аппаратов Самарского университета, член Российской академии инженерных наук и постоянный участник Европейского семинара в области обучению проектированию авиационных конструкций (EWADE). Является автором более 250 научных работ, в том числе 5 книг. Область научных интересов - механика, теория и практика проектирования самолетов.

Valeriy A. Komarov (b. 1941). He graduated from Kuibyshev Aviation Institute in 1964, received his Doctor of Technical Sciences Degree in 1976, Full Professor Degree in 1978. He is Head of Aircraft Design Department at Samara University, Member of Russian Academy of Engineering Sciences and regular participant of the European Workshop on Aircraft Design Education (EWADE). He is the author of over 250 scientific papers including 5 books. His research interest is mechanics, theory and practice of aircraft structural design. 


\title{
ОЦЕНКА ДЛИНЫ ОБУЧАЮЩЕЙ ПОСЛЕДОВАТЕЛЬНОСТИ В ЗАДАЧЕ РАСПОЗНАВАНИЯ ОБРАЗОВ (БИОИНДИКАЦИЯ)
}

\author{
Г.С. Розенберг \\ Институт экологии Волжского бассейна РАН, Тольятти, Россия \\ genarozenberg@yandex.ru
}

\begin{abstract}
Аннотация
Задача создания системы распознавания образов распадается на ряд подзадач: формализации предметной области, формирования обучающей выборки, обучения системы распознавания, снижения размерности пространства признаков, собственно задача распознавания (по степени сходства распознаваемого объекта с обучающей выборкой), контроля качества распознавания, адаптации, обратной задачи распознавания, кластерного и конструктивного анализа, когнитивного анализа. В статье рассматривается формализация одной из подзадач (формирования обучающей выборки). С помощью предложенной вероятностной модели сделан вывод о «почти линейной зависимости» длины обучающей последовательности и размерности пространства признаков. Получена оценка длины обучающей последовательности для реалистичных значений параметров модели.
\end{abstract}

Ключевые слова: биоиндикачия, распознавание образов, длина обучающей последовательности, случайная величина, решающее правило.

Цитирование: Розенберг, Г.С. Оценка длины обучающей последовательности в задаче распознавания образов (биоиндикация) / Г.С. Розенберг // Онтология проектирования. - 2017. - Т. 7, №2(24). - C. 207-215. - DOI: 10.18287/2223-9537-2017-7-2-207-215.

\section{Введение}

Содержательная (а не формальная) постановка задачи распознавания образов появилась в конце 50-х годов прошлого века и заключалась в том, чтобы построить систему, способную обучаться классификации ситуаций так же, как это делают живые существа. Такое широкое понимание проблемы привело к возникновению различных направлений этих исследований $[1,2]:$ одни считали главным построение модели восприятия [3], другие видели проблему в том, чтобы используя априорные сведения о свойствах образов, найти такое их описание, при котором отыскание принципа классификации не составляет труда [4], третьи понимали задачу распознавания образов как задачу минимизации риска в специальном классе решающих правил [5], четвёртые определяли распознавание образов как процесс, не требующий точного описания и основанный на обнаружении сходства объектов [6-8], а в работе [9] в деятельности по распознаванию образов находят сходство с процессом проектирования объектов. Эта «пестрота» подходов к задаче распознавания образов объясняется тем, что разные авторы различно представляют как само понятие «образ», так и процесс распознавания. Главным препятствием, стоящим на пути исследователей в этой обширной области, является отсутствие чёткого понимания того, какие процессы происходят при обучении человека.

Распознавание представляет собой информационный процесс, реализуемый некоторым преобразователем информации (системой распознавания), имеющим вход и выход. На вход системы подаётся информация о том, какими признаками обладают предъявляемые объекты; на выходе системы отображается информация о том, к каким классам (обобщённым образам) 
отнесены распознаваемые объекты. При создании и эксплуатации автоматизированной системы распознавания образов решается ряд задач.

Рассмотрим кратко основные из них [10]:

1) задача формализации предметной области (задача кодирования);

2) задача формирования обучающей выборки (база данных, содержащая описание объектов в пространстве признаков, дополненная информацией о принадлежности этих объектов к определенным классам распознавания);

3) задача обучения системы распознавания (обучающая выборка используется для формирования обобщенных образов классов распознавания);

4) задача снижения размерности пространства признаков (после обучения системы распознавания можно определить для каждого признака его ценность для решения задачи распознавания; наименее ценные признаки могут быть удалены из системы признаков; затем система распознавания должна быть обучена заново и этот процесс может повторяться, т.е. быть итерационным);

5) собственно задача распознавания (по степени сходства распознаваемого объекта с обучающей выборкой);

6) задача контроля качества распознавания (адекватность; определение фактической средней вероятности ошибки по всем классам распознавания, а также вероятности ошибки при отнесении распознаваемого объекта к определённому классу; результаты распознавания должны интерпретироваться с учётом имеющейся информации о качестве распознавания);

7) задача адаптации (если контроль качества распознавания неудовлетворителен, то необходимо переформатировать распознаваемую и обучающую выборки, вновь решить задачу обучения системы распознавания, стремясь повысить адекватность классификации распознаваемых объектов);

8) обратная задача распознавания (для данного класса распознавания системой устанавливается, какие признаки наиболее характерны для объектов данного класса);

9) задачи кластерного и конструктивного анализа (результатом кластерного анализа является классификация объектов по кластерам);

10) задача когнитивного анализа (анализ пространства признаков по сходству классов распознавания: наличие одного признака у разных классов распознавания или различных признаков у разных классов вносит определённый [интерпретируемый] вклад в их сходство и различие).

В данной статье более подробно формализуется и решается задача 2.

\section{1 Оценка длины обучающей последовательности}

Задача распознавания образов «с учителем» может быть сформулирована в следующем виде: пусть для $l$ объектов $\left\{o_{i}\right\}(i=\overline{1, l}$; назовем их обучающчей последовательностью) известна принадлежность каждого объекта к одному из классов $\left\{A_{j}\right\}$, где $j=\overline{1, k}$. Каждый объект задается набором $N$ признаков и, следовательно, может быть представлен точкой в $N$-мерном пространстве признаков $X$. В пространстве $X$ строится разделяющая функция (решающее правило) $\Phi$ таким образом, чтобы она разбила $X$ на $k$ непересекающихся подпространств $\left\{X_{j}\right\}$, в каждом из которых будут находиться объекты $\left\{o_{i}\right\}$ только одного класса; например, для $k=2$ имеем: $\Phi\left(o_{i}\right) \geq 0$ для $o_{i} \in A_{1}$ и $\Phi\left(o_{i}\right)<0$ для $o_{i} \in A_{2}$, где $A_{1} \cup A_{2}, A_{1} \cap A_{2}=\varnothing$. Следует ожидать, что качество решающего правила, представляющее собой степень соответствия построенного разбиения пространства $X$ на подпространства $\left\{X_{j}\right\}$ действительным классам $\left\{A_{j}\right\}$, 
должно быть функцией длины обучающей последовательности $l$, размерности пространства признаков $N$ и числа классов обучения $k$, то есть $\Phi=\Phi\left(l, N, k, o_{i}\right)$.

Существует мнение, что в качестве исходных признаков необходимо задавать всё, что только можно заподозрить в информативности. С другой стороны, замечено, что при практическом решении задач распознавания образов увеличение размерности пространства признаков не только не улучшает качество распознавания, но, в некоторых случаях, ухудшает его $[11,12]$. Отсюда возникает задача оценки длины обучающей последовательности в зависимости от размерности пространства признаков $N$. Приводимое в литературе решение этой задачи $[13,14]$ содержит лишь грубую оценку, что не позволяет воспользоваться полученными результатами во многих теоретических и прикладных работах. Данная статья ставит своей целью получение более точных формул решения этой задачи.

Пусть $Z$ - некоторая случайная величина, элементарными событиями которой являются $\left\{Z_{i}\right\}$ с вероятностями $\left\{p_{i}\right\}$, и пусть $S$ - некоторая совокупность элементарных событий из множества $\left\{Z_{i}\right\}$. Пусть предстоит провести $l$ независимых повторных наблюдений над случайной величиной $Z$. Через $v_{A}^{(l)}=n_{A} / l$ обозначим частоту появления события $A \in S$ для некоторой выборки длиной $l$, где $n_{A}-$ число элементов выборки, принадлежащих $A$. Классическая теорема Бернулли утверждает, что при фиксированном событии $A$ последовательность $v_{A}^{(l)}$ подчиняется биномиальному закону распределения и сходится к $p(A)$ по вероятности при $l \rightarrow \infty$, то есть для любого $\varepsilon>0$ справедливо

$$
P\left\{\left|v_{A}^{(l)}-P(A)\right|>\varepsilon\right\} \underset{l \rightarrow \infty}{\longrightarrow} 0 .
$$

Обозначим через $Y(l)=\sup _{A \in S}\left|v_{A}^{(l)}-P(A)\right|$ случайную величину, представляющую собой наибольшее уклонение частоты $v_{A}^{(l)}$ от вероятности события $A$ по всем $A \in S$. Если для любого $\varepsilon>0$ справедливо

$$
P\{Y(l)>\varepsilon\} \underset{l \rightarrow \infty}{\longrightarrow} 0,
$$

то можно говорить о равномерной сходимости частоты к своей вероятности [15-17]. Событие $Y(l)>\varepsilon$ произойдет в том случае, если хотя бы для одного из событий $A_{j} \in S$, где $j=\overline{1, T}(T-$ число наблюдений за реализацией $Z$ в классе $\left.A_{j}\right)$, будет справедливо $\left|v_{A}^{(l)}-P(A)\right|>\varepsilon$. Так как наблюдения над $Z$ проводятся независимо друг от друга, используя теорему о сложении вероятностей $[15,17]$, получим:

$$
P\{Y(l)>\varepsilon\} \leq \sum_{j=1}^{T} P\left\{v_{A}^{(l)}-P\left(A_{j}\right) \mid>\varepsilon\right\} \leq T \cdot P\left\{\left|v_{A}^{(l)}-P(A)\right|>\varepsilon\right\},
$$

где в качестве $A$ взято событие, для которого $P\left\{\left|v_{A}^{(l)}-P\left(A_{j}\right)\right|>\varepsilon\right\}$ принимает наибольшее значение. Учитывая, что $v_{A}^{(l)}$ подчиняется биномиальному закону распределения, при больших $l$ справедлива следующая оценка [6]:

$$
P\left\{\left|v_{A}^{(l)}-P(A)\right|>\varepsilon\right\} \leq \sqrt{\frac{2 P(A)[1-P(A)]}{\pi l \varepsilon^{2}} \exp \left(-\frac{l \varepsilon^{2}}{2 P(A)[1-P(A)]}\right) .}
$$

Подставляя эту оценку в (1) и полагая $P(A)=P$, получим: 


$$
P\{Y(l)>\varepsilon\} \leq T \cdot \sqrt{\frac{2 P(1-P)}{\pi l \varepsilon^{2}}} \exp \left(-\frac{l \varepsilon^{2}}{2 P(1-P)}\right) .
$$

Если потребовать, чтобы вероятность $P\{Y(l)>\varepsilon\}$ не превосходила некоторого достаточно малого $\delta$, то из уравнения

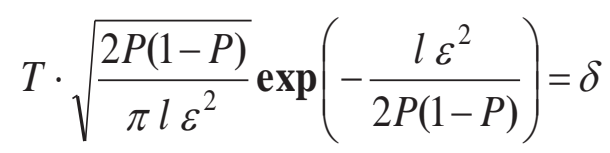

при заданных $P$ и $\varepsilon$ можно определить $l$.

Проведённые выше рассуждения справедливы для фиксированного конечного $T$. Если представить $T$ как некоторую функцию длины выборки $l$ и размерности пространства элементарных событий $N$, то есть $T=T(l, N)$, то условие равномерной сходимости (1) будет выполнено только в том случае, если $T=T(l, N)$ будет возрастать с ростом $l$ медленнее, чем $\exp \left(\frac{l \varepsilon^{2}}{2 P(1-P)}\right)$

В общем случае, если не учитывать вид решающего правила, число всевозможных классификаций $l$ объектов на $k$ классов будет $k^{l}$, так как каждый объект может принадлежать любому из $k$ классов. Однако в зависимости от положения объектов в пространстве $X$ и от вида решающего правила $\Phi$, число классификаций может быть значительно меньше. В ряде работ $[18,19]$ получена точная формула числа линейных дихотомий, то есть числа разбиения совокупности объектов на два класса с помощью гиперплоскостей:

$$
T_{2}(l, N)=\left\{\begin{array}{c}
2^{l}, \quad \text { для } N \geq l, \\
2 \cdot \sum_{i=0}^{N} C_{l-1}^{i}, \quad \text { для } N<l .
\end{array}\right.
$$

Таким образом, подставляя в (2) значение $T=T_{2}(l, N)$ для $l>N$, получим

$$
\sum_{i=1}^{N} C_{l-1}^{i}=\frac{\delta \varepsilon}{2} \sqrt{\frac{\pi l}{2 P(1-P)}} \exp \left(\frac{l \varepsilon^{2}}{2 P(1-P)}\right) .
$$

Следовательно, с вероятностью $1-\delta$ можно утверждать, что частота правильной классификации $N$-мерных объектов с помощью гиперплоскости будет отличаться от вероятности $P$ не более, чем на $\varepsilon>0$, если длина обучающей последовательности $l>l_{0}$, где $l_{0}$ является решением уравнения (3). Анализ этой связи позволяет сделать вывод о «почти линейной зависимости» длины обучающей последовательности и размерности пространства признаков. Например, для $\delta=0,2, P=0,95, \varepsilon=0,2$ и $N<250$ можно считать $l \approx 6 N$.

В работе [20] показано, что необходимость в разделении объектов кусочно-линейными поверхностями возникает лишь в самых сложных случаях взаимного расположения этих объектов и, следовательно, полученная оценка является завышенной. Это подтверждает результаты вычислительных экспериментов по редукции длины обучающей последовательности, представленные в $[21,22]$. Таким образом, в зависимости от степени «компактности» распознаваемых объектов в пространстве признаков в практической работе можно пользоваться числом $l$ из интервала $3 N \leq l \leq 6 N$ (для указанных выше $\delta, P$ и $\varepsilon$ ). 


\section{2 Экологическая интерпретация (биоиндикация)}

Содержание принципа антропоцентризма исторически менялось, исходя из понимания сущности человека (в рамках гуманитарных представлений различных философских школ и учений). Однако он всегда находился «во главе угла» в отношении истории развития нормирования (наложение граничных условий [нормативов] как на само воздействие, так и на факторы среды, отражающие и воздействие, и отклики экосистем): значительно ранее прочих были установлены нормативы приемлемых именно для человека условий среды (прежде всего, производственной). Тем самым было положено начало работам в области санитарногигиенического нормирования. Однако человек - не самый чувствительный из биологических видов и принцип «защищён человек - защищены и экосистемы», вообще говоря, неверен. Таким образом, экологическое нормирование является ключевой проблемой в формировании экологической безопасности.

В принципе, можно изучать фактор среды, проводя его прямые измерения-анализы. Однако, если для некоторых факторов эти измерения сравнительно просты (например, высота над уровнем моря или интенсивность $\gamma$-излучения), то для других представляют значительную сложность (например, засолённость почвы) или просто невозможны при однократном наблюдении (увлажнение почвы или изменяющаяся со временем концентрация некоторого загрязнителя в водной среде). Кроме того, как бы мы ни снижали уровень отрицательного воздействия, например, на водные массы, инструментальными методами невозможно контролировать присутствие всех загрязнителей. Занятие это очень трудоёмкое и финансово крайне затратное. Нужен постоянный контроль качества водной среды, а его может обеспечить только гидробиологический мониторинг. С другой стороны, связь между живыми организмами и факторами среды носит случайный характер и для её изучения необходимо привлекать вероятностное моделирование и строгие статистические методы.

Экологическое нормирование не является подменой санитарно-гигиеническому нормированию, а, в определённом смысле, дополняет его, ужесточая применяемые стандарты. Например, экологическая индикация может дать сведения о степени и характере загрязнения, распределении загрязнения в водоёме, возможном состоянии водной экосистемы в сезонном масштабе. Из этого следует, что вода, качество которой согласно экологическому контролю признано неудовлетворительным, вряд ли может использоваться для питьевых или хозяйственных целей, но экологически доброкачественная вода не всегда может быть признана пригодной с точки зрения здравоохранения. В последнем случае необходимы специфические микробиологические, токсикологические и химические тесты.

Одной из основ экологического нормирования является задача распознавания факторов среды по видам или группам видов-индикаторов (например, по растительности - геоботаническая индикация, фитоиндикация), актуальность которой не вызывает сомнений. Правда, почти полвека тому назад, крупнейшие фитоценологи отмечали наметившийся «застой» в количественных методах: «Как ни странно, но задачи фитоиндикации, вероятностные по своей природе, до сих пор решаются в основном без использования каких-либо статистических методов» [23, с. 141] и «Репрезентативные выборки, где независимо учитываются объект индикации и индикаторы, и строго статистические методы обработки данных - единственно возможный путь дальнейшего прогресса фитоиндиакационных исследований» [24, с. 1342]. С сожалением приходится констатировать, что ситуация за это время в биоиндикационных исследованиях кардинально не поменялась [25].

Среди причин объективного характера сложившейся ситуации можно назвать тот факт, что экосистемам, чаще всего, свойственен континуум по факторам среды и, следовательно, деление фактора на градации - процесс условный. В какой-то степени, нам «нельзя говорить о наилучшем (или оптимальном) решении, что, наоборот, характерно для задач в замкнутой 
форме... При решении задач в открытой форме используются не только (и не столько) верифицируемые знания, главное для которых - их доказуемая истинность, но и аксиологические ценностно ориентированные знания» [26, с. 13]. Это обосновывает применение онтологического подхода $[27,28]$ в задачах распознавания образов, а проведённая выше оптимизация длины обучающей последовательности позволяет «объективизировать» процесс биоиндикации (в частности, геоботанической индикации факторов среды по растительности [29, 30]).

\section{Заключение}

Следует признать, что как и любая сложная система (сложный процесс), в которой участвует Человек [31], процесс биоиндикации (распознавания образов) не может быть полностью формализован без учёта онтологических принципов. Включение Человека со всем багажом его знаний в систему распознавания образов «на равных» (замена субъектобъектных отношений на субъект-субъектные [26]) способно придать задаче биоиндикации большую концептуализацию предметной области. «Однако "знать", это ещё не значит "делать". Поэтому приходится придерживаться ещё и этического рационализма, согласно которому в основе поведения людей лежит (или должно лежать) рациональное начало; соответственно, знание о том, как необходимо поступать, является в данном случае достаточным условием нормативного поведения (выделено автором - Г.Р.)» [26, с. 15]. В качестве «рационального начала» в этой статье и выступает оценка длины обучающей последовательности в процедуре распознавания образов.

\section{Благодарности}

Автор благодарен профессорам Б.С. Флейшману, В.А. Виттиху и С.В. Смирнову за обсуждение некоторых проблем системологии. Работа выполнена при частичной финансовой поддержке Российского гуманитарного научного фонда (грант 16-13-63004-Самара) и Российского фонда фундаментальных исследований (грант 17-44-630113).

\section{Список источников}

[1] Сойфер, В.A. Методы компьютерной обработки изображений / В.А. Сойфер (ред.). - М.: Физматлит, 2001. $-784 \mathrm{c.}$

[2] Донской, В.И. Алгоритмические модели обучения классификации: обоснование, сравнение, выбор / Донской В.И. - Симферополь: ДИАЙПИ, 2014. - 228 с.

[3] Сочивко, В.П. Электронные опознающие устройства / Сочивко В.П. - М.: Энергия, 1964. - 56 с. (Сер.: Библиотека по автоматике. Вып. 91).

[4] Трапезников, В.A. Кибернетика и автоматическое управление / В.А. Трапезников // Вестн. АН СССР. 1962. - № 5. - C. 33-42.

[5] Вапник, В.Н. Теория распознавания образов (теоретические проблемы обучения) / В.Н. Вапник, А.Я. Червоненкис. - М.: Наука, 1974. - 416 с.

[6] Бонгард, М.M. Моделирование процесса узнавания на цифровой счетной машине / М.М. Бонгард // Биофизика. - 1961. - Т. 4, № 2. - С. 17-23.

[7] Браверман, Э.М. О методе потенциальных функций / Э.М. Браверман // Автоматика и телемеханика. 1965. - Т. 26, № 12. - С. 2205-2213.

[8] Айзерман, М.A. Теоретические основы метода потенциальных функций в задаче об обучении автоматов разделению входных ситуаций на классы / М.А. Айзерман, Э.М. Браверман, Л.И. Розоноэр // Автоматика и телемеханика. - 1964. - Т. 25, № 6. - С. 917-936.

[9] Боргест, Н.M. Распознавание образов при создании артефактов как метафора и как прикладные технологии онтологии проектирования / Н.М. Боргест // Онтология проектирования. - 2015. - Т.5, №1(15). - С. 1929. 
[10] Симанков, В.C. Адаптивное управление сложными системами на основе теории распознавания образов / В.С. Симанков, Е.В. Луценко - Краснодар: Кубан. гос. технол. ун-т, 1999. - 318 с.

[11] Харкевич, $\boldsymbol{A}$.A. О выборе признаков при машинном опознании / А.А. Харкевич // Изв. АН СССР, сер. техн. киберн. - 1963. - № 2. - С. 3-9.

[12] Kanal, L.N. On dimensionality and sample size in statistical pattern classification / L.N. Kanal, B. Chandrasekaran // Pattern Recognition. - 1971. - V. 3, No. 3. - P. 225-234.

[13] Вапник, В.Н. Об одном классе алгоритмов обучения распознаванию образов / В.Н. Вапник, А.Я. Червоненкис // Автоматика и телемеханика - 1964. - Т. 25, № 6. - С. 937-945.

[14] Бабу, К.Ч. О применении потенциальной функции Башкирова, Бравермана и Мучника для выделения информативных признаков при распознавании образов / К.Ч. Бабу , С.Н. Калра // Автоматика и телемеханика. - 1972. - Т. 33, № 12. - С. 105-107.

[15] Смирнов, Н.В. О приближении плотностей распределения случайных величин / Н.В. Смирнов // Уч. зап. МГПИ им. В.П. Потемкина. - 1951. - Т. 16, вып. III. - С. 69-96.

[16] Слуцкий, E.E. Избранные труды: Теория вероятностей. Математическая статистика / Е.Е. Слуцкий - М.: Изд-во АН СССР, 1960. - 291 с.

[17] Вапник, В.Н. О равномерной сходимости частот появления событий к их вероятностям / В.Н. Вапник, А.Я. Червоненкис // Докл. АН СССР (ДАН), - 1968. - Т. 181, вып. 4. - С. 781-784.

[18] Нильсон, Н. Обучающие машины / Н. Нильсон. - М.: Мир, 1967. - 180 с.

[19] Флейшман, Б.С. Элементы теории потенциальной эффективности сложных систем / Б.С. Флейшман. - М.: Сов. радио, 1971. - 224 с.

[20] Лецкий, Э.К. Оптимизация решений при проектировании сетей сбора данных / Э.К. Лецкий // Вестн. МИИТ. - 1998. - Вып. 1. - С. 125-130.

[21] Розенберг, Г.С. Опыт приложения теории распознавания образов для оценки засоления почв по растительности / Г.С. Розенберг, Б.М. Миркин , С.Ю. Рудерман // Экология. - 1972. - № 6. - С. 31-34.

[22] Розенберг, Г.С. Редукция числа признаков и эффективность оценки почв по растительности при использовании методов распознавания образов / Г.С. Розенберг // Количественные методы анализа растительности: Материалы IV Всесоюз. совещания по проблеме «Применение количественных методов в анализе структуры растительности». - Уфа: БФАН СССР, 1974. - С. 36-39.

[23] Василевич, В.И. Второе совещание «Применение количественных методов при изучении структуры растительности» (Тарту, 1969) / В.И. Василевич // Ботан. журн. - 1970. - Т. 55, № 1. - С. 140-142.

[24] Миркин, Б.М. Рецензия «Теоретические вопросы фитоиндикации» / Б.М. Миркин // Ботан. журн. - 1972. Т. 57, № 12. - С. 1342-1344.

[25] Шитиков, В.К. Количественная гидроэкология: методы, критерии, решения: в 2-х кн. / В.К. Шитиков, Г.С. Розенберг, Т.Д. Зинченко. - М.: Наука, 2005. - Кн. 1. 281 с.; Кн. 2. 337 с.

[26] Bummux, B.A. Парадигма ограниченной рациональности принятия решений: препринт / В.А. Виттих - Самара: ИПУСС РАН, 2009. - 26 с.

[27] Смирнов, С.B. Онтологический анализ предметных областей моделирования / С.В. Смирнов // Известия Самарского НЦ РАН. - 2001. - Т. 3, № 1. - С. 62-70.

[28] Смирнов, С.В. Онтологическое моделирование в ситуационном управлении / С.В. Смирнов // Онтология проектирования. - 2012. - № 2 (4). - С. 16-24.

[29] Розенберг, Г.С. Модели в фитоценологии / Г.С. Розенберг. - М.: Наука, 1984. - 240 с.

[30] Розенберг, Г.С. Введение в теоретическую экологию: в 2-х т.; изд. 2-е, исправ. и дополненное / Г.С. Розенберг. - Тольятти: Кассандра, 2013. - Т. 1. 565 с.; Т. 2. 445 с.

[31] Розенберг, Г.С. О простых, сложных и суперсложных системах / Розенберг Г.С. // Четверта міжнародна науково-практична конференція «Відкриті еволюціо-нуючі системи» (20-21 травня 2016 р.). Збірник праць: Частина 1. - Ніжин: ВНЗ ВП НУБіП України НАI, 2016. - С. 228-233. 


\title{
THE ESTIMATE OF THE LENGTH OF THE TRAINING SEQUENCE IN THE TASK OF PATTERN RECOGNITION (BIOINDICATION)
}

\author{
G.S. Rozenberg \\ Institute of Ecology of the Volga River Basin of the RAS, Togliatti, Russia \\ genarozenberg@yandex.ru
}

\begin{abstract}
Development of a pattern recognition system is comprised from a number of subtasks: formalization of the subject area, formation of a training sample, training of the recognition system, reduction of the dimensionality of the feature space, the problem of pattern recognition itself (based on the degree of similarity of the detected object with the training sample), recognition quality control, adaptation, inverse problem recognition, cluster and structural analysis, cognitive analysis. The article considers the formalization one of the subtasks (formation of the training sample). The conclusion about the "almost linear dependence" of the length of the training sequences and the dimensionality of the feature space is made using the proposed probabilistic model. Evaluation of the length of the training sequences for realistic values of model parameters is performed.
\end{abstract}

Key words: bioindication, pattern recognition, the length of training sequences, a random variable, the decision rule.

Citation: Rozenberg GS. Estimation of the length of the training sequence in the problem of pattern recognition (bioindication). Ontology of designing. 2017; 7(2): 207-215. DOI: 10.18287/2223-9537-2017-7-2-207-215.

\section{References}

[1] Soifer VA. Methods of Computer Image Processing [In Russian]. - Moscow: Fizmatlit, 2001. - 784 p.

[2] Donskoy VI. Algorithmic models of learning classification: rationale, comparison, selection [In Russian]. - Simferopol: DIP, 2014. - 228 p.

[3] Sochivko VP. Electronic Identifying Device [In Russian]. - Moscow: Energiya, 1964. - 56 p.

[4] Trapeznikov VA. Cybernetics and automatic control [In Russian]. Vestn. Acad. of Sci. of the USSR. - 1962. No. 5. - P. 33-42.

[5] Vapnik VN., Chervonenkis AYa. Theory of Pattern Recognition (Theoretical Problems of Studying) [In Russian]. - Moscow: Nauka, 1974. - 416 p

[6] Bongard MM. Modeling of the recognition process on the digital counting machine [In Russian]. Biophysics. 1961. - V. 4, No. 2. - P. 17-23.

[7] Braverman EM. On the method of potential functions [In Russian]. Automation and Remote Control. - 1965. V. 26, No. 12. - P. 2205-2213.

[8] Aizerman MA., Braverman EM., Rozonoer LI. Theoretical fundamentals of the method of potential functions in the problem of teaching machines to split the input into classes of situations [In Russian]. Automation and Remote Control. - 1964. - V. 25, No. 6. - P. 917-936.

[9] Borgest NM. Pattern recognition in designing artifacts is as metaphor and as an applied technology of ontology of designing [In Russian] Ontology of designing. 2015; 5(1): 19-29.

[10] Simankov VS., Lutsenko EV. Adaptive Control of Complex Systems Based on the Theory of Pattern Recognition [In Russian]. - Krasnodar: Kuban. Tekhnol. Univ, 1999. - 318 p.

[11] Kharkevich AA. On the choice of features for machine identification [In Russian]. Izv. Acad. of Sci. of the USSR, ser. tech. cybern. - 1963. - No. 2. - P. 3-9.

[12] Kanal LN., Chandrasekaran B. On dimensionality and sample size in statistical pattern classification. Pattern Recogn. - 1971. - V. 3, No. 3. - P. 225-234.

[13] Vapnik VN., Chervonenkis AYa. On a class of algorithms for pattern recognition learning [In Russian]. Automation and Remote Control. - 1964. - V. 25, No. 6. - P. 937-945.

[14] Babu KCh, Kalra SN. About the use of potential functions Bashkirov, Braverman and Muchnik for the selection of informative features in pattern recognition [In Russian]. Automation and Remote Control. - 1972. - V. 33, No. 12. - P. 105-107. 
[15] Smirnov N.V. On the approximation of densities of distribution of random variables [In Russian]. Scientific Notes VP. Potemkin's MGPI. - 1951. - V. 16, No. III. - P. 69-96.

[16] Slutsky EE. Selected Works: Theory of Probability. Mathematical Statistics [In Russian]. - Moscow: Acad. of Sci. of the USSR Press, 1960. - 291 p.

[17] Vapnik VN., Chervonenkis AYa. On uniform convergence of frequencies of occurrence of events to their likely professions [In Russian]. Dokl. Acad. of Sci. of the USSR (DAS). - 1968. - V. 181, No. 4. - P. 781-784.

[18] Nilsson N. Learning Machines: Foundations of Trainable Pattern-Classifying Systems. - N. Y.: McGraw-Hill, 1965. $137 \mathrm{p}$.

[19] Fleishman BS. Elements of the Theory of Potential Effectiveness of Complex Systems [In Russian]. - Moscow: Sov. Radio, 1971. - 224 p.

[20] Lecky EK. Optimization solutions for the design of network data collection [In Russian]. - Vestn. MIIT. - 1998. V. 1. - P. 125-130.

[21] Rozenberg GS., Mirkin BM., Ruderman YuS. Experience in applications of the theory of pattern recognition for the evaluation of soil salinity on vegetation [In Russian]. - Rus. J. Ecology. - 1972. - No. 6. - P. 31-34.

[22] Rozenberg GS. Reduction of the number of features and the evaluation of the soils for vegetation, when using methods of pattern recognition [In Russian]. - Quantitative Methods of Vegetation Analysis: Proceedings of the IV All-Union Conference "The Application of Quantitative Methods in the Analysis of Vegetation Structure". Ufa: Acad. of Sci. of the USSR Press, 1974. - P. 36-39.

[23] Vasilevich VI. Second meeting "Application of quantitative methods in the study of the research Institute structure of vegetation" (Tartu, 1969) [In Russian]. - Bot. J. - 1970. - V. 55, No. 1. - P. 140-142.

[24] Mirkin BM. The Review "Theoretical Issues of Phytoindication" [In Russian]. - Bot. J. - 1972. - V. 57, No. 12. P. 1342-1344.

[25] Shitikov VK., Rozenberg GS., Zinchenko TD. Quantitative Hydroecology: Methods, Criteria, Solutions: $2^{\text {nd }}$ Vol. [In Russian]. - Moscow: Nauka, 2005. - V. 1. 281 p.; V. 2. 337 p.

[26] Vittikh VA. Paradigm of Bounded Rationality Decision-Making: Preprint [In Russian]. - Samara: ICSCS RAS Press, 2009. - 26 p.

[27] Smirnov SV. Ontological analysis of the subject areas modeling [In Russian]. - Izv. Samar. SC RAS. - 2001. V. 3, No. 1. - P. 62-70.

[28] Smirnov SV. Ontological modeling in situational management [In Russian]. - Ontology of Designing. - 2012. No. 2 (4). - P. 16-24.

[29] Rozenberg GS. Models in Phytocenology [In Russian]. - Moscow: Nauka, 1984. - 240 p.

[30] Rozenberg GS. Introduction to Theoretical Ecology. $2^{\text {nd }}$ Vol., Ed. $2^{\text {nd }}$. [In Russian]. - Toglyatti: Kassandra, 2013. V. 1. 565 p.; V. 2. 445 p.

[31] Rozenberg GS. On simple, complex, and supercomplex systems [In Russian]. - Fourth International ScientificPractical Conference "Open Evaluation Systems" (May 20-21, 2016). Proceedings: Part 1. - Nizhyn (Ukraine): Univ. Press, 2016. - P. 228-233.

\section{Сведения об авторе}

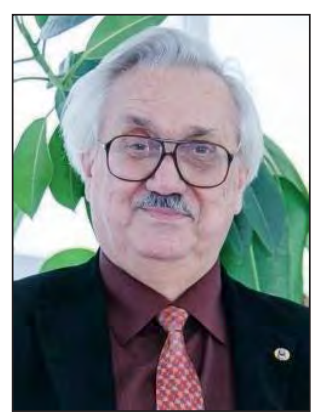

Розенберг Геннадий Самуилович, 1949 г. рождения. Окончил Башкирский государственный университет (физико-математический и биологический факультеты) в 1966 г. Д.б.н., профессор, член-корреспондент РАН (с 2000 г.). Директор Института экологии Волжского бассейна РАН (с1989 г.). Опубликовал более 800 научных работ (в том числе более 60 монографий) в области теоретической экологии и моделирования экосистем.

Gennady S. Rozenberg (b. 1949). Graduated from the Bashkir State University (physicmathematical and biological faculties; 1966). Doctor of biological Sciences, Professor, corresponding member of the Russian Academy of Sciences (2000). Director of the Institute of Ecology of the Volga River Basin of the RAS (1989). He has published more than 800 scientific papers (including more than 60 monographs) in the field of theoretical ecology and ecosystem modeling. 


\title{
УДК 681.3.01.016:006:354
}

\section{ОНТОЛОГИИ УПРАВЛЕНИЯ КАЧЕСТВОМ КОМПЬЮТЕРНЫХ ДАННЫХ ОБ ИЗДЕЛИИ}

\section{П.С. Шильников}

Московский государственный технический университет имени Н.Э. Баумана (национальный исследовательский университет), Москва, Россия Peter.Shilnikov@gmail.com

\begin{abstract}
Аннотация
Расширение применения компьютерной техники в информационном сопровождении жизненного цикла изделия приводит к возникновению и росту проблем, вызванных зависимостью компьютерных документов от средств интерпретации этих документов. В статье рассмотрено качество компьютерных данных об изделии (PDQ - Product Data Quality). Под качеством данных об изделии понимается отсутствие дефектов качества данных. Дефект качества данных - это существование таких особенностей компьютерной модели изделия, которые препятствуют последующей обработке модели. Под управлением качеством компьютерных данных понимается чередование транзакций обнаружения и устранения дефектов качества данных. Сформулированы основные задачи, решение которых будет способствовать повышению качества компьютерных данных конструкторской документации об изделии. Для повышения качества компьютерных данных об изделии необходимо определить влияние дефектов качества данных на выполнение последующей обработки компьютерных документов, взаимосвязь дефектов качества данных, последовательность транзакций управления качеством данных и возможность искажения модели изделия в результате устранения дефектов качества данных. Новым в статье является комплексный подход к задаче управления качеством компьютерных данных об изделии. При формулировании задач использовались публикации, результаты, полученные по итогам НИОКР, а также опыт многолетней эксплуатации и усовершенствования программных модулей проверки, исправления и импорта файлов в CAD-системы APM Studio.
\end{abstract}

Ключевые слова: качество, компьютерные данные, качество данных об изделии, системы автоматизации проектирования, управление качеством данных.

Цитирование: Шильников, П.С. Онтологии управления качеством компьютерных данных об изделии / П.С. Шильников // Онтология проектирования. - 2017. - Т. 7, №2(24). - С. 216-226. - DOI: 10.18287/2223-9537-2017-7-2-216-226.

\section{Введение}

Исследуемая проблема возникла в процессе выполнения научно-исследовательской и опытно-конструкторской работы [1], базируется на опыте многолетней эксплуатации и усовершенствования программных модулей проверки, исправления и импорта файлов в CADсистемы APM Studio [2], а также на результатах исследований [3, 4], включая стандарт [5].

Документы являются средствами, необходимыми на всех этапах жизненного цикла изделия (ЖЦИ). Можно ли считать документ моделью? Рассмотрим понятие «модель». Модель это объект, дающий ответы на вопросы о другом, моделируемом объекте [6]. На различных этапах ЖЦИ используются многообразные модели изделия:

- рабочий чертёж или CAD-модель содержит данные об устройстве изделия и его свойствах;

- технологический процесс (в любой форме: в виде заполненных бумажных бланков, в виде записей в базе данных и т.д.) содержит сведения об изготовлении изделия; 
- инструкция по эксплуатации (как в бумажной форме, так и в форме ИЭТР - интерактивных электронных технических руководств) содержит информацию об использовании изделия; и т.д.

Модель, имеющая уникальное обозначение и прошедшая некоторую последовательность согласований и утверждений, является документом. При документировании процессов ЖЦИ большое значение имеют утверждённые подлинники документов.

В настоящее время в процессах ЖЦИ возрастает роль компьютерной документации [7]. Особенностью компьютерного документа является то, что если бумажный документ доступен для непосредственной интерпретации человеком, интерпретация компьютерного документа требует аппаратных и программных компьютерных средств. При переходе на повсеместное применение компьютерных документов возникают три проблемы, требующие решения: интеграция компьютерных данных, обеспечение качества компьютерных данных и обеспечение долгосрочного хранения компьютерных данных. Поскольку все три проблемы имеют общий источник, проблемы взаимосвязаны [8]. Схематически такая связь показана на рисунке 1.

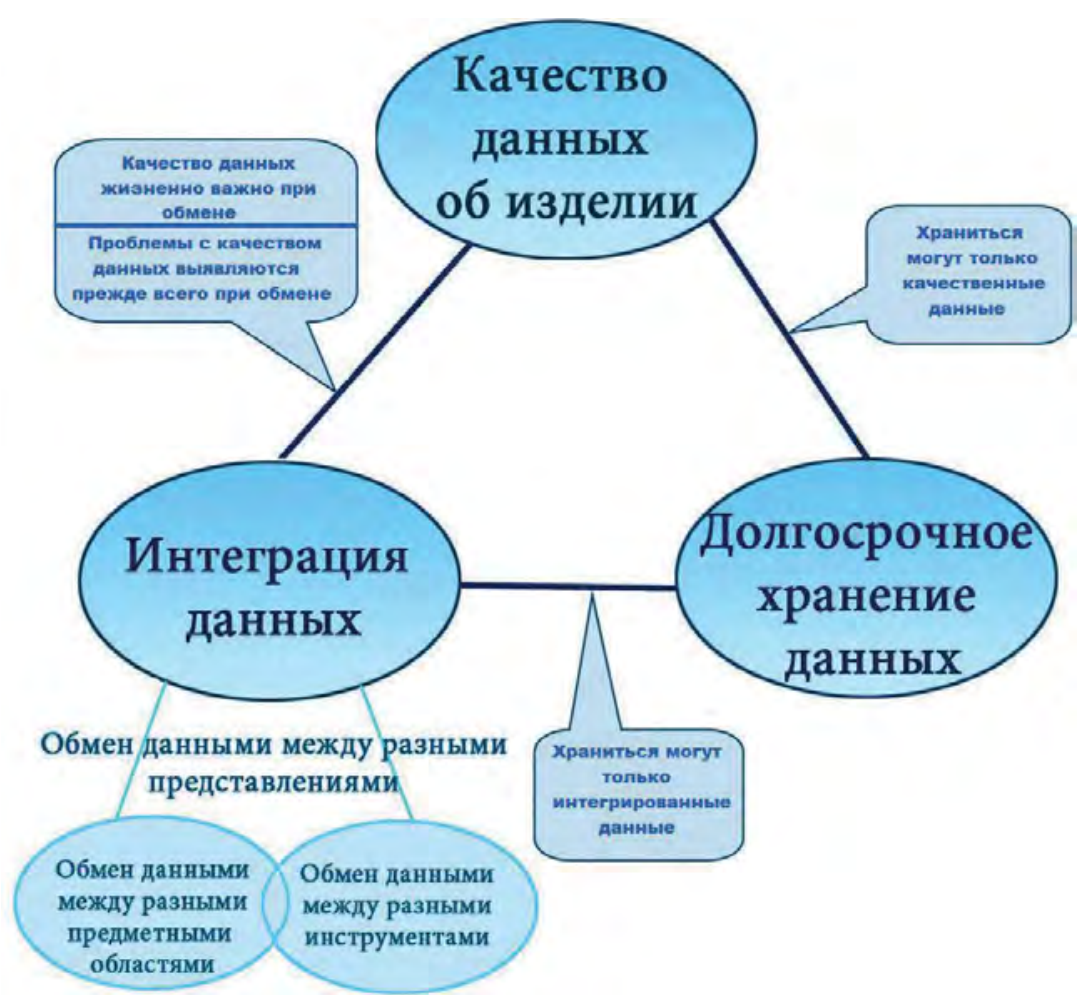

Рисунок 1 - Проблемы, вызванные зависимостью компьютерного документа от средств интерпретации

\section{1 Качество данных об изделии (PDQ - Product Data Quality)}

Под критерием качества данных понимается отсутствие соответствующего дефекта качества данных. Дефект качества компьютерных данных - это такое свойство компьютерного документа, которое затрудняет использование документа для последующей его обработки компьютерными средствами [8].

Компьютерный документ, имеющий неприемлемое качество содержащихся в нём данных, непригоден для полноценного последующего использования. Затраты на обеспечение качества данных компьютерного документа составляют около $20 \%$ всех затрат на создание документа [6]. Качество данных компьютерного документа может влиять на качество модели изделия и через него - на качество самого изделия, как это показано на рисунке 2. Возможно 
и непосредственное влияние качества данных на качество изделия [9]. Следовательно, любое усовершенствование процессов управления качеством данных приводит к существенному сокращению затрат.

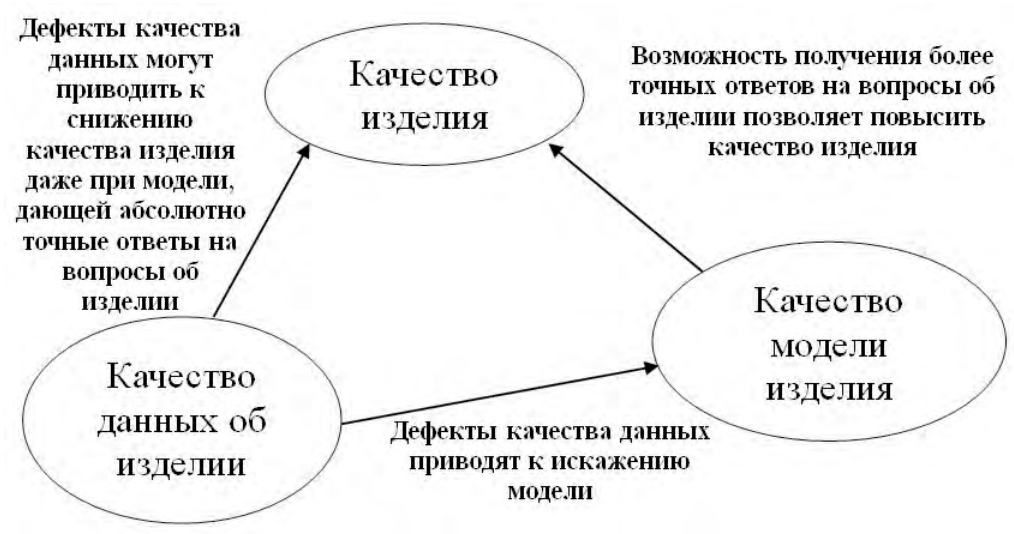

Рисунок 2 - Связь качества изделия, качества модели изделия и качества данных об изделии [9]

Если компьютерный документ утверждён, а тем более - защищён электронной подписью, изменение компьютерного документа с целью устранения дефектов качества данных, превращается в серьёзную проблему:

- в любом случае внесение изменений в компьютерный документ может привести к искажению содержания документа;

- защищённый документ может изменяться только уполномоченными участниками процесса.

Вследствие вышесказанного, качество данных компьютерного документа должно быть обеспечено до его утверждения.

Для различных предметных областей разработаны наборы критериев качества данных для содержащих данные об изделии компьютерных документов. Критерии качества данных приведены в документах VDA4955, ISO/PAS 26183 SASIG PDQ, ISO 10303-59 PDQS.

Рассмотрим дефекты качества данных для механических CAD-систем. Такие модели содержат данные о структуре изделия, геометрические и топологические данные, определяющие форму изделия, и др.

Процесс управления качеством данных представляет собой последовательность действий по обнаружению и устранению дефектов качества данных. Под управлением качеством данных об изделии (PDQM Product Data Quality Management) будем понимать согласованную совокупность работ:

- $\quad$ по проверке качества данных об изделии, $C=\left\{c_{i}\right\}$;

- по устранению выявленных дефектов качества, $H=\left\{h_{i}\right\}$;

- по регистрации выполненных действий, $R=\left\{r_{i}\right\}$.

Здесь индекс $i$ соответствует некоторому $i$-му критерию качества.

Тогда деятельность по управлению качеством данных можно представить как:

$A_{P D Q M}=\{C, H, R\}$ или $A_{P D Q M}=\left\{c_{\mathrm{i}}, h_{\mathrm{j}}, r_{\mathrm{k}}\right\}$.

Опыт выполнения работ по управлению качеством электронных данных показывает высокую затратность и низкую надёжность процессов «лечения» (healing) CAD-моделей, т.е. устранения обнаруженных дефектов качества. В настоящее время существует большое число программных инструментов для обнаружения и устранения дефектов качества данных в CAD-моделях. Такие инструменты реализованы одним из нижеперечисленных способов:

- модули, входящие в штатную комплектацию CAD-систем, иногда эти модули автоматически запускаются при импорте сторонних моделей изделия в CAD-систему, в некото- 
рых случаях эти же или другие модули могут быть запущены по требованию пользователя;

- дополнительные (optional) модули CAD-систем (Q-Checker для CATIA v5 и др.);

- независимые программные продукты (CADFix, CADDoctor, TransVidia и др.).

Часто работа этих программных инструментов выполняется автоматически, и пользователь не может влиять на их работу. Иногда у пользователя есть возможность управлять работой инструментов, задавая:

- набор выполняемых действий;

- допустимые значения свойств модели (например, наименьшие допустимые длины крошечных рёбер, наименьшие допустимые ширины узких граней, допустимые расстояния от точек, которые должны лежать на поверхностях, до поверхностей и т.д.);

- последовательность выполняемых действий.

\section{2 Задачи организации процесса управления качеством данных}

\section{1 Определение значимости дефектов качества в зависимости от предполагаемого дальнейшего использования документа}

Можно привести следующие примеры различной значимости дефектов.

- Наличие разрывов второй производной (кривизны) в геометрических моделях нежелательно для фрагментов изделий, взаимодействующих с потоками жидкости и газа (трубопроводы, внешние обводы воздушных и водных транспортных средств и т.д.) и изделий, подвергающихся скоростной обработке резанием. В геометрических моделях, используемых для построения конечно-элементной модели, этот критерий несущественен.

- Наличие в геометрической модели «крошечных» рёбер приводит к искажению конечноэлементной модели, как это показано на рисунке 3, и к созданию неэффективных траекторий режущего инструмента. При изготовлении изделия посредством аддитивных технологий, когда форма детали аппроксимируется большим числом мелких элементов, данный дефект несущественен.

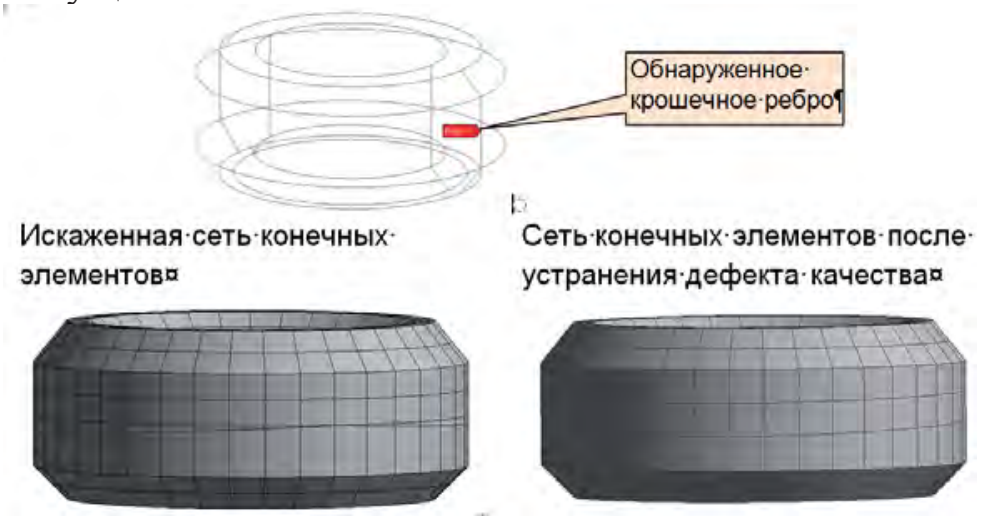

Рисунок 3 - Влияние наличия крошечного ребра на конечно-элементную модель

Создание методик, однозначно, точно и полно определяющих значимость всех дефектов качества данных, имеет огромную практическую значимость, т.к., с одной стороны, позволит не тратить ресурсы на обнаружение и устранение несущественных дефектов качества данных, и, с другой стороны, не даст пропустить обнаружение и устранение таких дефектов качества, наличие которых может вызвать серьёзные трудности при выполнении работ.

Из вышеприведённых примеров становится очевидным, что определение «значимости» или «критичности» дефектов качества данных CAD-модели имеет смысл только в том слу- 
чае, если учитывается то, для решения каких задач будет в последующем использоваться CAD-модель.

Решение задачи требует классификации дефектов качества данных с построением онтологии. За основу могут быть взяты система кодирования критериев качества из ISO/PAS 26183 SASIG PDQ (построенная на принципах фасетной классификации Ранганатана) [5] и концептуальные схемы данных из ISO 10303-59 PDQS. Также должна быть построена онтология задач. По результатам сопоставления этих онтологий могут быть созданы методики. Очевидно, сопоставление разнородных онтологий станет нелегкой задачей.

\section{2 Определение взаимосвязей дефектов качества}

По этому вопросу можно привести некоторые соображения, сделанные на основе «здравого смысла». Взаимосвязи дефектов качества можно рассматривать в статическом и динамическом аспектах.

В статическом аспекте можно привести следующие примеры: наличие крошечных рёбер, крошечных и узких граней взаимосвязаны. Крошечная грань всегда имеет контур, состоящий из крошечных рёбер. Некоторые узкие грани являются крошечными гранями, это зависит от соотношения допустимых значений, применяемых при обнаружении этих дефектов качества данных. Часто узкие грани имеют в контурах крошечные рёбра.

В динамическом аспекте необходимо учитывать то, что устранение некоторых дефектов качества данных может привести как к исчезновению некоторых других дефектов качества данных (например, удаление крошечной грани повлечет удаление крошечных рёбер, образующих контур удалённой крошечной грани), так и к появлению новых дефектов качества данных (например, удаление крошечного ребра связано с заменой крошечного ребра одной вершиной, при этом велика вероятность того, что новая вершина будет находиться на неприемлемом расстоянии от смежных рёбер и граней).

\section{3 Определение последовательности действий по обнаружению и устранению дефектов качества данных}

В общем случае эта последовательность может быть итерационной. Исходными данными здесь должны служить выявленные в динамическом аспекте взаимосвязи дефектов качества.

Прежде всего, последовательность выполняемых действий имеет смысл в том случае, если управление качеством данных включает не только обнаружение дефектов качества данных, но и устранение обнаруженных дефектов. Также можно упорядочить выполняемые проверки по степени значимости дефектов качества. В этом случае при обнаружении критических («фатальных») дефектов качества данных дальнейшие проверки не имеют смысла, и их можно прекратить.

\section{4 Создание компьютерной поддержки процесса управления качеством данных}

В настоящее время процедура управления качеством данных осуществляется следующим образом: проверяемая CAD-модель импортируется в систему проверки (модуль CADсистемы или независимый программный продукт), после чего выполняется проверка и создается отчёт об обнаруженных дефектах качества данных. В некоторых случаях для дефекта качества данных указывается обозначение (идентификатор) фрагмента модели, в котором обнаружен дефект качества данных (например, при импорте обменного файла STEP в NX в протоколе импорта содержатся номера записей в обменном файле). Отметим, что:

- во всех известных случаях, кроме постпроцессора STEP CAD-системы APM Studio, проверяется не исходная CAD-модель, а модель, полученная в результате импорта 
исходной модели в систему проверки; при импорте возможны искажения исходной модели и (или) появление новых дефектов качества;

- отчёт об обнаруженных дефектах качества данных создаётся в виде текстового файла, предназначенного для чтения человеком, а не для программной обработки;

- в некоторых системах пользователь может видеть обнаруженные дефекты качества данных на экране.

В последние годы возникли следующие предпосылки:

- в проекте долгосрочного хранения компьютерных данных об изделии LOTAR International устанавливается, что данные должны сохраняться в формате ISO 10303 STEP;

- в одном из основных Прикладных протоколов STEP, которые будут использоваться в проекте LOTAR International, Протоколе ISO 10303-242, появилась возможность представлять данные об обнаруженных дефектах качества непосредственно в CADмодели. На рисунке 4 показан пример представления такого дефекта.

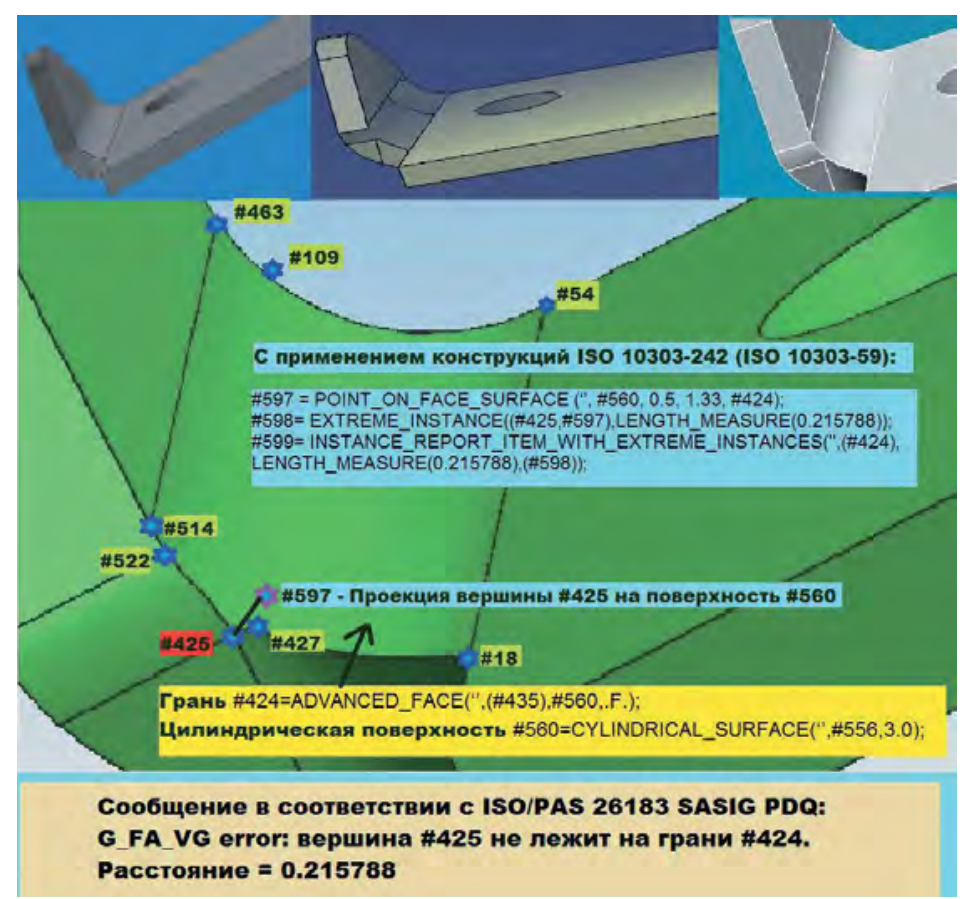

Рисунок 4 - Представление обнаруженного дефекта качества данных на основе онтологии ISO 10303-242 [8]

Первая из предпосылок приводит к тому, что если утверждённым компьютерным документом (подлинником или «источником мастер-данных») является файл в формате ISO 10303 STEP, то и дефекты качества данных разумнее обнаруживать в файле STEP, а не в его отображении, полученном в результате импорта в систему проверки. Тем более, что в файле, соответствующем Протоколу ISO 10303-242, можно хранить не только модель изделия, но и результаты проверки качества данных модели.

Перспективным является хранение информации не только об обнаруженном дефекте качества, но и о выполненных процедурах исправления дефекта [10].

Следует учитывать, что:

- устранение дефектов качества может привести как к появлению новых дефектов качества, иногда - более серьёзных, так и к искажению модели; 
- новые дефекты качества данных и искажения модели могут возникнуть не непосредственно при устранении дефекта качества данных, а в ходе последующей обработки модели.

Полезной была бы возможность вернуться к предыдущему состоянию модели (то, что аналогично «откату» в базах данных). Для реализации данной возможности требуется доработка Протокола ISO 10303-242.

\section{5 Разработка методики проверки искажения CAD-моделей}

Качество модели изделия, т.е. её полнота, точность и достоверность, и качество данных об изделии взаимосвязаны. Требуется исследование этой взаимосвязи.

Искажения CAD-модели, ведущие к понижению её достоверности, и, следовательно, к снижению качества модели, могут возникать как в результате конвертации (преобразования) модели, так и в результате устранения дефектов качества данных модели.

\subsection{1 Искажение в результате конвертации}

На рисунке 5 показано изменение модели простейшего изделия в результате передачи модели из одной CAD-системы в другую CAD-систему. Единственный неаналитический элемент в рассматриваемом случае - кромка отверстия в цилиндре, получаемая в результате пересечения двух цилиндров. В общем случае (когда радиусы цилиндров не равны) линия пересечения двух цилиндров является кривой четвёртого порядка. Из рисунка 5 видно, что в одной из систем контур отверстия состоит из двух рёбер, в другой - из четырёх рёбер.

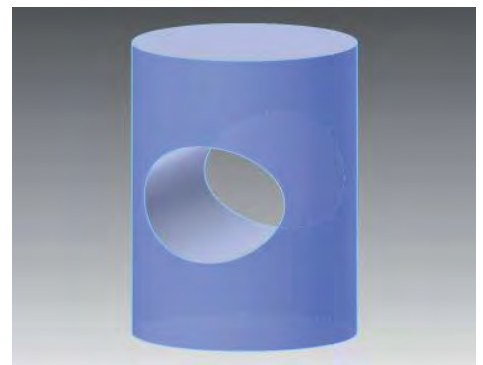

a

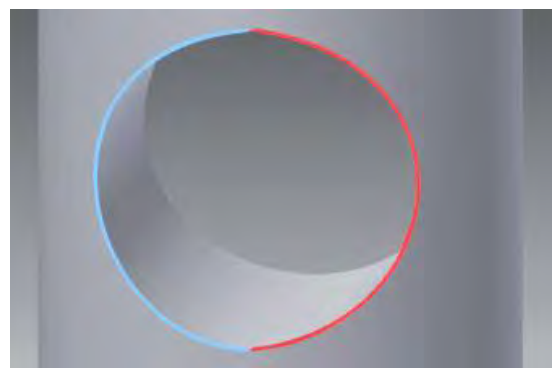

6

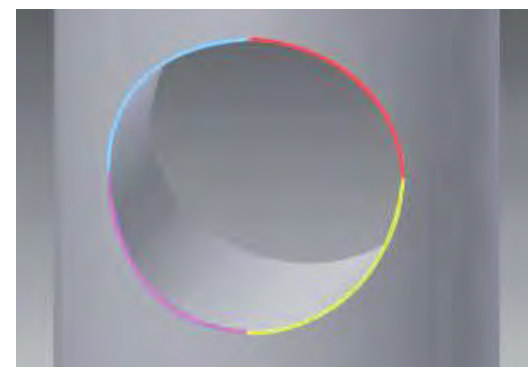

B

a) - общий вид модели, б) - контур грани в исходной CAD-системе состоит из двух рёбер,

в) - контур грани после импорта в принимающую CAD-систему состоит из четырёх рёбер

Рисунок 5 - Представление линии пересечения двух цилиндров в различных CAD-системах

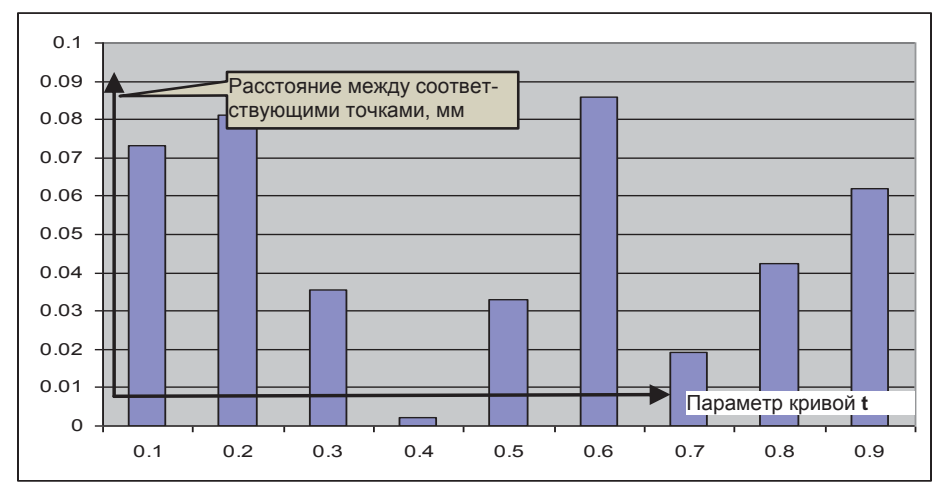

Рисунок 6 - Расстояния между соответствующими точками (мм) на линиях пересечения двух цилиндров в различных CAD-системах (параметр кривой $t$ )
На рисунке 6 показаны различия положений (абсолютные) соответствующих точек на кривой в двух моделях изделия в различных CAD-системах. При равных значениях параметра $t$ отличия в положениях точек на кривой пересечения в разных моделях составляют до 0.1 мм (менее $1 \%$ от габаритных размеров). 


\subsection{2 Искажение в результате устранения дефекта качества данных}

На рисунке 7 показана модель изделия, содержащая дефект качества. При загрузке модели не удаётся построить одну из граней. В протоколе импорта появляется запись: «!Ошибка - Сбой CRTOBY - Код ошибки: 15116 - Не-манифолд вершина \#207» и подобные записи ещё о трёх вершинах. В компьютерной геометрии понятие «манифолд» имеет формальное определение [11]. К не-манифолд элементам относятся такие элементы, как стенки нулевой толщины, отверстия нулевого диаметра, самопересекающиеся кривые и поверхности, контуры кривых, касающиеся в одной точке или пересекающиеся. С математической точки зрения такие элементы вызывают трудности при их обработке штатными алгоритмами. С практической точки зрения такие элементы не могут существовать в вещественных объектах, в данном случае в детали, изготовленной по CAD-модели. На рисунке 8 показаны не-манифолд вершины, вызвавшие проблемы при построении изображения модели.

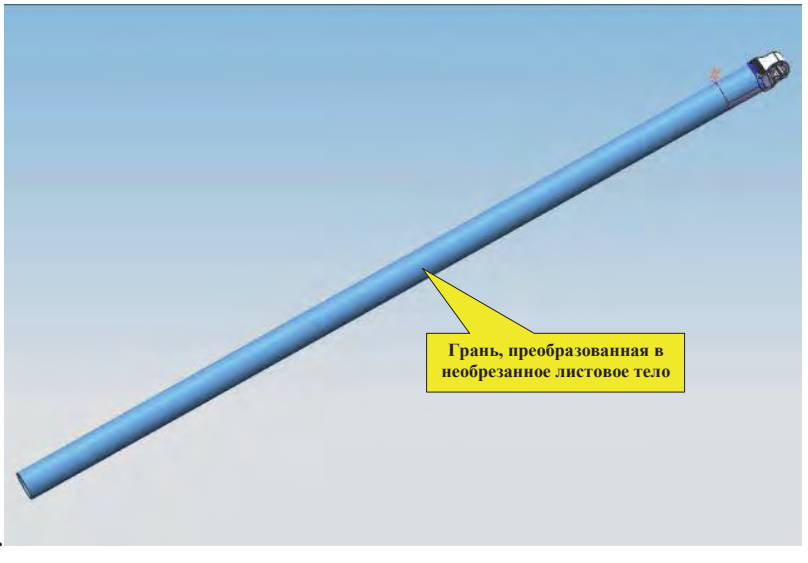

Рисунок 7 - Модель изделия, содержащая дефект качества

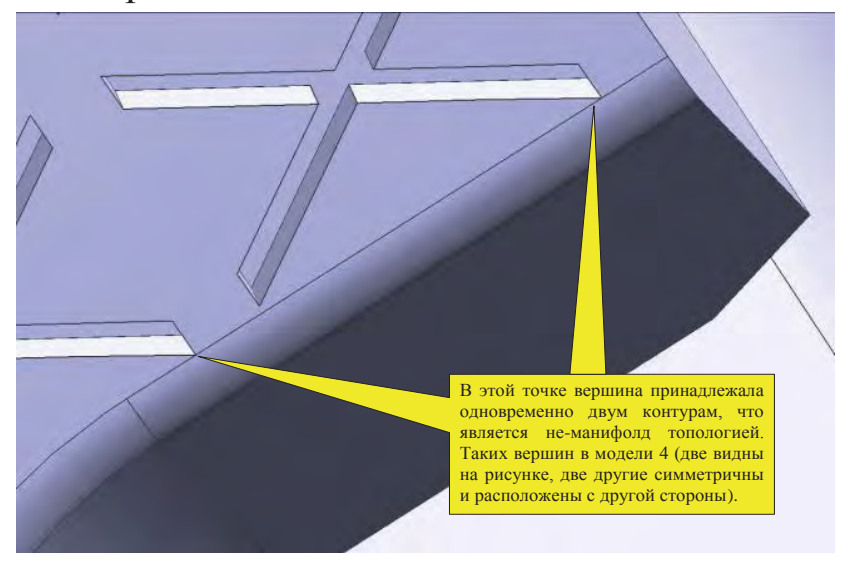

Рисунок 8 - Не-манифолд вершины

При исправлении твердотельной модели штатным модулем обнаружения и устранения дефектов качества, автоматически запускаемым при импорте сторонних моделей в одну из CAD-систем, обнаруженная проблема устраняется за счёт того, что пересекающийся внутренний контур отдаляется от внешнего контура (см. рисунок 9). В этом примере попытка повышение качества данных об изделии приводит к снижению качества модели изделия.

Проверка искажения модели геометрической формы изделия осуществляется сравнением модели с некоторой мастер-моделью (эталонной моделью). Мастер-моделями могут быть (в порядке возрастания полноты С):

1) Поверочные геометрические свойства (GVP - Geometric Validation Properties) - центр масс, площадь поверхности и объём изделия. Набор этих свойств может рассматриваться как одна из моделей изделия. Такая модель не даёт ответов на вопросы о геометрической форме изделия. Тем не менее, на ограниченный набор вопросов модель даёт ответы. Сравнение поверочных геометрических свойств с эталонными значениями позволяет сделать вывод о степени искажения модели геометрической формы изделия. Следует учитывать, что в общем случае для вычисления поверочных геометрических свойств используются численные алгоритмы, работающие с некоторой погрешностью, и для достаточно крупных изделий даже при очевидном искажении формы отклонения значений свойств могут быть сопоставимы с погрешностью численного алгоритма. Онтологии конструкторских протоколов STEP позволяют передавать с моделью поверочные геометрические свойства. Совпадение значений поверочных геометрических свойств является необходимым, но не достаточным условием отсутствия искажений геометрической модели. Пре- и 
постпроцессоры STEP CAD-систем NX и CATIA v5 обеспечивают экспорт и импорт поверочных геометрических свойств.

2) Облако точек (Point Cloud) - некоторые характерные точки модели, которые должны принадлежать рёбрам или граням. Облако точек также может рассматриваться как одна из

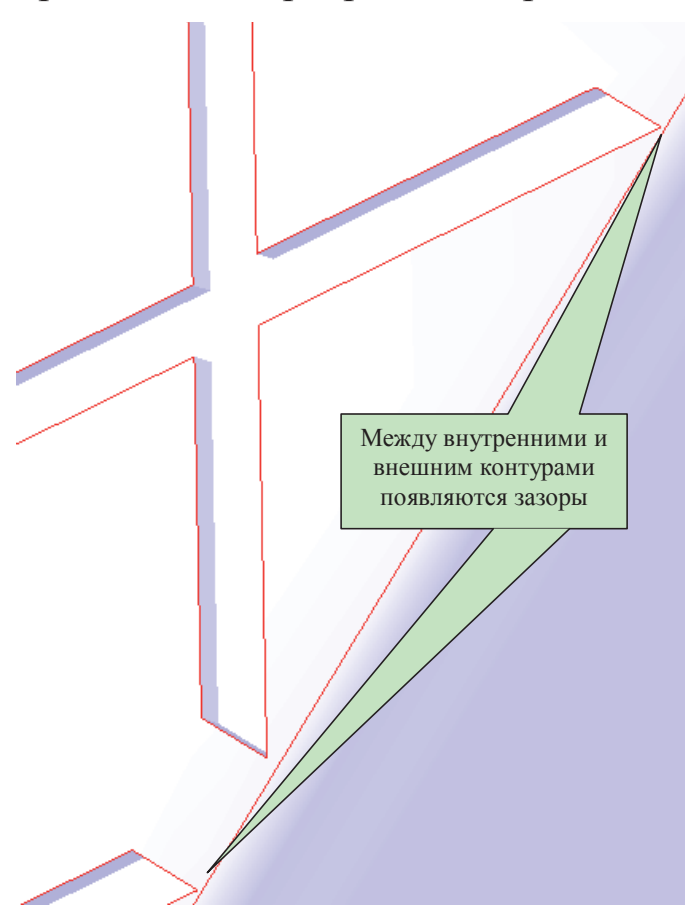

Рисунок 9 - Модель после исправления (устранения обнаруженного дефекта качества данных) моделей изделий, обеспечивающая ограниченный набор ответов на вопросы о геометрической форме изделия. Онтологии, позволяющие представлять облака точек, также имеются в конструкторских протоколах STEP. Проверка посредством облака обеспечивает более достоверное свидетельство отсутствия искажения модели. Надёжность такой проверки зависит от того, насколько верно подобрано множество поверочных точек.

3) Полная геометрическая модель формы, рассматриваемая в качестве эталонной. Проверка может выполняться, например, совмещением двух моделей формы или выполнением булевых операций над моделями. При этом следует учитывать то, что, по крайней мере, одна из моделей прошла процесс конвертации и могла быть искажена в ходе этого процесса. Онтологии поверочных геометрических свойств и облака точек просты, и вероятность искажения построенных по этим онтологиям моделей мала. В случае сравнения полных моделей имеются две модели, имеющие равную сложность и в равной мере подверженные искажениям.

Каждый из способов поиска искажений, основанный на мастер-моделях определённой разновидности, имеет свои достоинства и недостатки. В практической деятельности разумно применять сочетание этих способов.

Общее направление исследований обуславливает то, что рассматриваться должны искажения модели в результате устранения дефектов качества. Одна из задач, требующих решения - разделение искажений модели в результате конвертации от искажений в результате устранения дефектов качества.

\section{Заключение}

На основе опыта практической работы сформулированы задачи, решение которых позволит повысить результативность процесса обеспечения качества данных об изделии и снижение затрат, связанных с этим процессом. Отдельные положения содержатся в [12]. На основе существующих знаний могут быть созданы практические рекомендации.

Задача проверки искажения формы CAD-моделей может быть решена с помощью уже существующих на рынке программных продуктов. Необходимы практические рекомендации по внедрению такой проверки в процессы деловой деятельности [10]. В настоящее временя отсутствуют нормативные документы и программные продукты, обеспечивающие решение задачи записи протокола процедуры «лечения» CAD-модели формы изделия.

Онтологии, содержащиеся в прикладных протоколах стандарта ISO 10303 STEP, могут служить инструментом, используемым при решении упомянутых задач. Перспективным 
представляется освоение функциональных возможностей прикладного протокола АР242 STEP, которые предназначены для управления качеством данных об изделии.

\title{
Список источников
}

[1] Погосян, М.A. Повышение эффективности контроля качества геометрических данных электронных моделей изделий / М.А. Погосян, В.А. Злыгарев, Д.Ю. Стрелец, В.Н. Юрин, Б.В. Ященко // Технологические системы №3 (52) 2010. С. 20-22.

[2] Прокопов, В.C. APM Structure3D - новые возможности прочностного анализа / В.С. Прокопов, В.В. Шелофаст // САПР и графика, №11 2004. - С. 70-72.

[3] Марка, Д.А.. Методология структурного анализа и проектирования SADT. / Д.А. Марка, К. МакГоуэн // М: Эксмо-Пресс, 1999 - 243 с.

[4] Суханова, $\boldsymbol{A}$. "Отдавая должное инвестициям, технологиям и партнерам по внедрению PLM, мы осознавали, что главное условие успеха - увлеченность наших сотрудников" Интервью И.Ф. Гумерова, А.В. Пуртова и В.А. Авдеева / А. Суханова, Ю. Суханов // СAD/CAM/CAE Observer, № 1(85) 2014 С. 8-28.

[5] ISO/PAS 26183:2006. SASIG Product data quality guidelines for the global automotive industry.

[6] Thomas C. Redman. Data Quality: The Field Guide. Digital Press, 2001 - 241 p.

[7] Колчин, А.Ф. Управление жизненным циклом продукции / А.Ф. Колчин, М.В. Овсянников, А.Ф. Стрекалов, С.В. Сумароков // - М.: Анахарсис, 2002. - 304 с.

[8] Ренев, С.A. Треугольник ДеЛаПорта-Шильникова: Проблемы и решение / С.А. Ренев, Е.Л. Столярова, П.С. Шильников // САПР и графика, №1 2016. - С. 26-31.

[9] Ренев, $\boldsymbol{C}$. . Шерстяной треугольник качества / С.А. Ренев, Е.Л. Столярова, П.С. Шильников // САПР и графика, №7 2015. - С. 72-74.

[10] Shilnikov, $\boldsymbol{P}$. The ontology of healing of product shape data for ISO 10303 STEP. Proceedings of the 5th International Workshop on System \& Concurrent Engineering for Space Applications (SECESA). Lisbon, Portugal, 2012. - http://www.congrexprojects.com/docs/12c12_docs/0935-shilnikov.pdf?sfvrsn=2.

[11] Шильников, П.С. Представление данных КИП / П.С. Шильников. - 150 с. - http://www.plmconsulting.ru/pdf/cip_data_2009-11-01.pdf.

[12] Колчин, $\boldsymbol{A . \Phi . ~ П р е д с т а в л е н и е ~ м о д е л и ~ з н а н и и ̆ ~ с п е ц и а л и с т а ~ н а ~ о с н о в е ~ о н т о л о г и ч е с к о г о ~ п о д х о д а ~ / ~ А . Ф . ~ К о л - ~}$ чин, Н.В. Елисеева // Автоматизация. Современные технологии, №4, 2009. - С. 36-38.

\section{ONTOLOGIES OF PRODUCT DATA QUALITY MANAGEMENT}

\author{
P.S. Shilnikov \\ Bauman Moscow State Technical University (National Research University of Technology), Moscow, Russia \\ Peter.Shilnikov@gmail.com
}

\begin{abstract}
While expanding of computer usage for Product Life-cycle information support, several challenges, caused by dependence of computer documents upon interpretation means, are arising. Product data quality (PDQ) challenge is discussed in this article. Absence of data quality defects is assumed as product data quality. Data quality defect, in turn, is existence of some computer product model features, impeding downstream handling of product model. Product data quality management is assumed as interleaved transactions for detection and healing of data quality defects. The principal tasks that facilitate improvement of product design documents' quality were outlined. Computer product data quality is considered as one of aspects of quality of product design documents represented in computer form. The following tasks have to be solved for data quality improvement: detect influence of data quality defects on downstream handling of computer documents, detect interrelationship of data quality defects, set sequence of product data quality transactions, detect product shape model deviation arisen due to model healing. The new integrated approach to product data quality issues is implemented. Free accessible materials and also results of R\&D [1] and experience of multy-year exploitation and permanent improvement of APM Studio modules for quality check, healing and import of CAD models [2] were considered while outlining of principal tasks mentioned in this article.
\end{abstract}


Keywords: quality, computer data, product data quality, data quality management, computer aided design.

Citation: Shilnikov PS. Ontologies of product data quality management. Ontology of designing, 2017; 7(2): 216-226. DOI: 10.18287/2223-9537-2017-7-2-216-226.

\section{References}

[1] Pogosyan MA, Zlygarev VA, Strelets DY, Yurin VN, Yatshenko BV. Increasing the effectiveness of quality check of geometric data of electronic products models [In Russian]. Technological systems №3 (52) 2010. P. 20-22.

[2] Prokopov VS, Shelofast VV. Automated workstation Structure3D - new strength analysis capabilities [In Russian]. - M: CAD \& Graphics, №11. 2004. - p.70-72.

[3] Marca DA, McGowan C. SADT: structured analysis and design technique. McGraw-Hill Book Co., Inc.: New York, NY. 1988.

[4] Sukhanova A., Sukhanov Y. "Paying tribute to investments, technologies and partners for the implementation of PLM, we realized that the main condition for success is the enthusiasm of our employees" Interview with I.F. Gumerov, A.V. Purtov and V.A. Avdeev (OJSC KAMAZ) [In Russian]. CAD/CAM/CAE Observer, № 1(85) 2014. - P.8-28.

[5] ISO/PAS 26183:2006. SASIG Product data quality guidelines for the global automotive industry.

[6] Thomas C. Redman. Data Quality: The Field Guide. Digital Press, 2001 - 241 p.

[7] Kolchin AF, Ovsyannikov MV, Strekalov AF, Sumarokov SV. Product Life Cycle Management. [In Russian] M.: Anakharsis, 2002. 304 p.

[8] Renev S, Stolyarova C, Shilnikov P. The Triangle of DeLaPorte-Shilnikov: Challenges and Solution [In Russian]. CAD \& Graphics, №1 2016 г. p. 26-31.

[9] Renev S, Stolyarova C, Shilnikov P. Woolen Triangle Quality [In Russian]. CAD \& Graphics, №7, 2015, p.7274.

[10] Shilnikov $\boldsymbol{P}$. The ontology of healing of product shape data for ISO 10303 STEP. Proceedings of the 5th International Workshop on System \& Concurrent Engineering for Space Applications (SECESA). Lisbon, Portugal, 2012. - http://www.congrexprojects.com/docs/12c12_docs/0935-shilnikov.pdf?sfvrsn=2.

[11] Shilnikov P Representation of CIM data [In Russian]. - http://www.plm-consulting.ru/pdf/cip_data_2009-1101.pdf.

[12] Kolchin AF, Eliseeva NV. Specialist knowledge model presentation on the basis of ontological approach [In Russian]. Automation. Modern Technology, №4, 2009, p.36-38.

\section{Сведения об авторе}

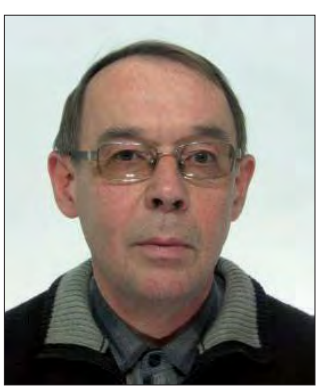

Шильников Петр Станиславович, 1956 г. рождения. Окончил Московское высшее техническое училище им. Н.Э. Баумана в 1979 г., к.т.н. (2006). Доцент кафедры компьютерных систем автоматизации производства Московского государственного технического университета им. Н.Э. Баумана (национального исследовательского университета). Специализируется по обмену данными между CAD-системами с применением формата ISO 10303 STEP и по качеству данных об изделии. В списке научных трудов около 50 работ в области автоматизации проектирования.

Peter Stanislavovich Shilnikov (b.1956) graduated from the Moscow Higher Technical School named after N.E. Bauman (Moscow) in 1979, PhD (2006). Hi is Associate Professor at Bauman Moscow State Technical University (Department of computer integrated manufacturing). His activity is focused on inter-CAD data exchange, primarily with ISO 10303 STEP format and on product data quality. Hi is co-author of about 50 scientific articles and abstracts in the field of CAD. 


\title{
Коммюнике Онтологического Саммита 2017 - ИСКУССТВЕННЫЙ ИНТЕЛЛЕКТ, МАШИННОЕ ОБУЧЕНИЕ, ЛОГИЧЕСКИЙ ВЫВОД И ОНТОЛОГИИ ${ }^{1}$
}

\author{
Kenneth Baclawski ${ }^{\mathrm{a}}$, Mike Bennett ${ }^{\mathrm{b}}$, Gary Berg-Cross ${ }^{\mathrm{c}}$, Donna Fritzsche ${ }^{\mathrm{d}}$, \\ Todd Schneider $^{\mathrm{e}}$, Ravi Sharma ${ }^{\mathrm{f}}$, Ram D. Sriram ${ }^{\mathrm{g}}$ and Andrea Westerninen ${ }^{\mathrm{h}}$ \\ ${ }^{a}$ Northeastern University, Boston, MA USA \\ ${ }^{\mathrm{b}}$ Hypercube Limited, London, UK \\ ${ }^{\mathrm{c}}$ RDA US Advisory Group, Troy, NY USA \\ ${ }^{\mathrm{d}}$ Hummingbird Design, Chicago, IL USA \\ ${ }^{\mathrm{e}}$ Engineering Semantics, Fairfax, VA USA \\ ${ }^{\mathrm{f}}$ Senior Enterprise Architect, Elk Grove, CA USA \\ ${ }^{\mathrm{g}}$ National Institute of Standards \& Technology, Gaithersburg, MD USA \\ ${ }^{\mathrm{h}}$ Nine Points Solutions
}

\begin{abstract}
Аннотация
Существует множество связей между искусственным интеллектом, обучением, логическим выводом и онтологиями. Онтологический саммит 2017 года был посвящён исследованию, выявлению и формулированию отношений между этими областями. Для популяризации онтологической науки в рамках сессий онтологического саммита 2017был выделен диалоговый инструментарий, что способствовало обсуждению и обмену знаниями между заинтересованными лицами. Результаты подтверждаются примерами из различных областей. Материал представлен в форме коммюнике, который дополнен ссылками на источники в Интернете.
\end{abstract}

Ключевые слова: искусственный интеллект, машинное обучение, логический вывод, онтологии.

\section{1 Введение}

В настоящее время наблюдается всё более широкое распространение элементов искусственного интеллекта (ИИ), который включает интеллектуальное поведение, работу с большими объёмами данных, обучение и адаптацию в вычислительных системах. Три из наиболее значимых технологии ИИ - это наличие всё большего количества данных (Big Data); быстро снижающаяся стоимость хранения и обработки данных; достижения в методах и средствах машинного обучения (MO) [1]. Это позволяет использовать сложные методы МО, которые требуют обработки больших объёмов данных, чтобы быть эффективными. Приложения МО «усилили распознавание речи Android, а также реализовали описанную в сериале Star Trek возможность мгновенного перевода в Skype. Google строит автомобили с автоматическим управлением, а компьютерные системы могут самостоятельно обучаться идентифицировать видео с кошками. Роботы-собаки ведут себя как настоящие» [2]. Стоит отметить, что, хотя они могут быть очень полезными, они являются типами так называемых «узких ИИ» технологий. Приложения ИИ позволяют компьютерам решать конкретные проблемы, такие как распознавание изображений, или выполнять логический вывод, которые не охватывают весь спектр свойственных человеку способностей к самоуправлению, познанию, называемых «общим ИИ» [1].

На Онтологическом саммите 2017 были предприняты попытки исследовать способы использования методов ИИ в области МО, рассуждений и онтологии для их взаимного улучшения. Эти области приложений были разделены на три направления (секции), но вскоре стало ясно, что разные секции имеют существенное пересечение друг с другом, и в каждой секции наблюдалось значительное разнообразие. «Обучение необходимо для приобретения знаний, однако логический вывод показывает нам лучшие способы хранения и доступа к знаниям. Таким образом, обучение и логический вывод являются взаимодополняющими, и их следует изучать совместно» [3].

\footnotetext{
${ }^{1}$ ONTOLOGY SUMMIT 2017 COMMUNIQU'E - AI, LEARNING, REASONING AND ONTOLOGIES https://s3.amazonaws.com/ontologforum/OntologySummit2017/Communique/OntologySummit2017Communique_v8.pdf.

Рекомендуемое цитирование в русскоязычной литературе:

Коммюнике Онтологического Саммита 2017 - Искусственный интеллект, машинное обучение, логический вывод и онтологии / Kenneth Baclawski, Mike Bennett, Gary Berg-Cross, Donna Fritzsche, Todd Schneider, Ravi Sharma, Ram D. Sriram and Andrea Westerninen. Пер. с англ. М.Д. Коровина // Онтология проектирования. - 2017. - Т. 7, №2(24). - C. 227-238. - DOI: 10.18287/2223-9537-2017-7-2-227-238.
} 
Было также отмечено, что термины «логический вывод» и «обучение» имеют много толкований. На онтологическом саммите было решено понятие «обучение» ограничить машинным обучением, поскольку контекст темы саммита был ИИ. Тем не менее, обучение человека также в определённой степени изучалось в рамках саммита, особенно в аспектах, касающихся МО. Термин «логический вывод» не должен ограничиваться формальными логическими рассуждениями. В целом на саммите было установлено, что классификация многих методов, определение передовых практик и выявление синергизма между технологиями МО, логическим выводом и онтологиями выявили три ключевые проблемы для использования отношений между ними.

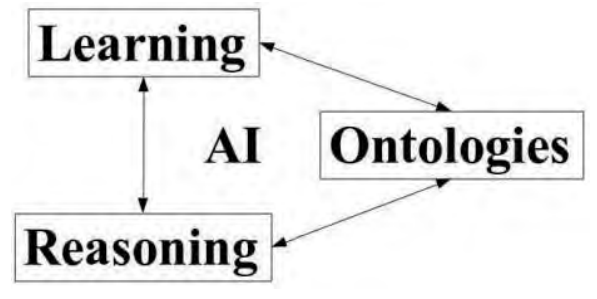

Рисунок 1 - Логотип Ontology Summit 2017
Саммит 2017 года рассмотрел современное состояние дел по основным темам ИИ: обучение, логический вывод и онтологии согласно трём направлениям, приведённым в разделе 2. Каждое направление было посвящено отношениям между двумя из трёх тем ИИ, отражённым на логотипе Ontology Summit 2017 и представленным на рисунке 1. Отношения между обучением и логическим выводом косвенным образом затрагивались онтологиями. Некоторые из основ темы саммита онтологий представлены в разделе 3 . В разделе 4 приводится обзор некоторых возможностей и проблем взаимоотношений между обучением, логическим выводом и онтологиями. Коммюнике заканчивается выводом и благодарностями.

\section{2 Содержание}

\section{1 Обзорная сессия}

Обзорная сессия открыла саммит презентацией, в которой были представлены многие темы, расширенные в последующих презентациях и дискуссиях. В частности, в обзоре представлена диаграмма онтологического обучения (Ontology Learning Layer Cake), показанная на рисунке 2, которая использовалась в качестве объединяющего элемента для всех направлений. Самый высокий уровень представляет логику и аксиомы, расположенные ниже слои содержат схемы, отношения, иерархии понятий и синонимы. Термины находятся на нижнем уровне. Диаграмма представляет собой основу для описания процесса извлечения знаний. Примером может служить процесс создания иерархии понятий, которая может быть изображена графами, представляющими отношения между элементами, как показано в [4,5].

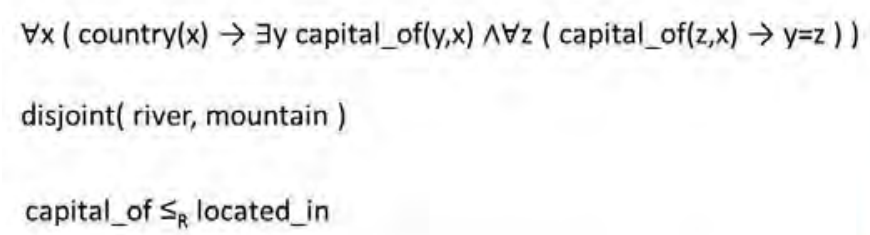

Рисунок 2 - Диаграмма онтологического обучения [4]

\section{2 Направление А: Применение МО для выделения знаний и улучшения онтологий}

По этому направлению рассматривалось одно из самых узких мест в ИИ; а именно, как создать достаточные знания о мире для действительно интеллектуального агента. До сих пор базы знаний и онтологии для таких 
целей создавались в значительной степени «вручную», что является малоэффективным с точки зрения потребных затрат времени и ресурсов. По этому направлению изучалось использование автоматизации и различных подходов МО для извлечения знаний и улучшения онтологий, в том числе их наполнения [6]. Ниже приведены некоторые из основных положений, представленных в этом направлении.

- Узкие места из-за сложности онтологического проектирования остаются проблемой, но были разработаны и могут быть использованы различные подходы и инструменты МО, в том числе статистические, лингвистические и биоподобные, которые могут быть использованы для того чтобы:

1) извлекать информацию и структурированные знания из различных источников для облегчения разработки и поддержки онтологий $[4,7,8]$;

2) фильтровать «шумные» данные для дальнейшего улучшения качества разработанных онтологий $[9,10]$;

3) гармонизировать онтологии для управления зависимостью от особенностей наборов данных [5];

4) осуществлять перевод лингвистических реализаций объектов онтологии с одного языка на другой [4].

- Диаграмма онтологического обучения обеспечивает концептуальную основу для обсуждения того, какие типы знаний строятся. Результаты, полученные автоматическими системами, как разработанные таксономии, сравниваются с лучшими имеющимися иерархиями, построенными вручную [11].

- Был достигнут значительный прогресс в области контролируемого обучения. Теперь этот подход применим за рамками сильно ограниченных доменов. Интересно, что есть также дополнительные перспективы от полу- и неконтролируемых подходов к обучению, которые предлагают возможность избежать проблем, связанных с потребностью в большом количестве учебных наборов данных. Однако, может потребоваться контроль учебных наборов, как это было в случае с обучением языку Never-Ending (NELL), а также совокупным использованием знаний для поддержки обучения [7].

- В некоторых областях, таких как биомедицинские, есть достаточные условия для получения новых знаний, используя МО, качественные онтологии и множество предметных данных о генах и функциях клеток [12].

\section{3 Направление В: Использование фоновых знаний для улучшения результатов МО}

Задача этого направления - выявить проблемы и возможности в «использовании фоновых знаний для улучшения результатов МО», роли онтологий и сопоставимых ресурсов для достижения этой цели и требований для онтологий, которые могут быть использованы этими способами. Пять докладчиков представили примеры и идеи, имело место оживлённое обсуждение рассмотренных вопросов и идей. В рамках заседаний были представлены семантические, синтаксические и контекстуальные аспекты МО с использованием онтологий. Были обсуждены пересечения с темами других направлений, а также общие идеи и возможности [13]. Ниже приведены некоторые из основных моментов презентаций и обсуждений в рамках этой секции.

- Фоновые знания полезны для того, чтобы сделать результаты МО понятными [1], а также имитировать человеческие качества настроения и интуиции $[14,15]$.

- Существует ошеломляющее множество вариантов моделей и их комбинаций [13].

- Фоновые знания могут улучшить качество результатов МО, используя методы логического вывода для выбора моделей обучения и подготовки данных обучения и тестирования (сокращение больших, шумных наборов данных до управляемых, сфокусированных) [15,16,17].

- Онтологии, используемые для улучшения результатов НЛП, не обязательно должны быть такими же онтологиями, как те, которые синтезируются инструментами НЛП. Действительно, они могут быть даже иного типа [13]. Задача состоит в том, чтобы обеспечить совместимость онтологий, чтобы можно было итеративно улучшить одну и ту же онтологию, используя эти два действия.

- Контекст важен для устранения неоднозначных терминов [18].

- Сочетание онтологического проектирования с МО может улучшить поддержку принятия решений, в том числе улучшить качество решений, сделать более понятным причины принятия решения и адаптировать процесс принятия решений к изменяющимся условиям [19].

- Бизнес-онтология финансовой индустрии (Financial Industry Business Ontology - FIBO) и корпоративные таксономии могут помочь извлечь и интегрировать информацию из хранилищ данных, оперативной памяти и обеспечить коммуникацию на естественном языке [18].

- Важно не только иметь возможность извлекать графы знаний из многоязычного текста, но также обеспечивать многоязычность онтологии [4].

\section{4 Направление C: Использование онтологий для логического вывода и обратно}

Цель направления С состояла в том, чтобы обсудить методы, разработанные для рассуждений с использованием онтологических основ. Любой интеллектуальный агент имеет четыре основных компонента [20]:

1) датчики, которые принимают внешние сигналы в различных формах; 
2) знания, которые проявляются в различных формах (например, качественные, количественные и сочетания обоих);

3) механизмы вывода, которые делают заключения о мире, учитывая входные данные и используя знания;

4) исполнительные механизмы, которые выполняют различные действия (например, физические и умственные).

Однако, человеческий интеллект также требует наличия:

1) механизмов обратной связи, включая способность изучать новое поведение [19,21];

2) анализа эмоций и настроений $[14,15]$;

3) социального поведения [15].

Ниже приводятся некоторые из основных моментов презентаций и обсуждений в рамках этого направления.

- Онтологии составляют ядро для представления знаний, которое используется соответствующей стратегией вывода [22].

- Существует много форм стратегий вывода (например, обратный и прямой выводы, нестрогие рассуждения, удовлетворение ограничениям, доказательство теорем) [22].

- Постепенно добавляя аксиомы (выбор окружения), можно улучшить скорость вывода [22].

- Важными являются также другие механизмы вывода, в том числе вероятностные [5,9] и использующие аналогии [23].

- Онтологическая совместимость варьируется от минимальной выразительности (например, таксономии) на синтаксическом уровне до промежуточного (например, тезауруса) на структурном уровне с различными уровнями выразительности (например, концептуальных моделей и логической теории) и до самого высокого семантического уровня с логикой первого порядка. См. Рисунок 2.

- Инструменты и технологии также следуют этой последовательности [22].

- Логический вывод может помочь устранить неопределённость терминов в перекрывающихся предметных областях (ПрО) [24].

- Онтологии могут помочь в открытии научных знаний и могут помочь автоматизировать процесс открытий. Анализ рабочего процесса может помочь понять результаты процесса научных открытий [21].

- Примеры проектирования, применимые для вывода онтологии, касаются структурных стратегий, требований и вариантов использования, включая требования к экосистеме [23].

- Онтологии могут быть использованы для нахождения аналогов в биологической области для решения проблем в инженерной области [23].

\section{3 Истоки}

Первые успехи с машинным переводом, а также машинное «обучение» с использованием статистических методов, предполагали, что некоторый прогресс может быть сделан субсимвольным образом, т. е. без конкретных представлений о знаниях [25], в более поздних методах ИИ также преобладает субсимвольное МО. Однако субсимвольное МО обычно работает, решая проблемы классификации или регрессии на неинтерпретированных исходных данных. Системы, разработанные для решения этих проблем, можно сказать, «учатся» в смысле оптимизации набора параметров модели для повышения производительности с течением времени. Называя это «обучением», мы представляем процесс как когнитивный и мысленный, но, как правило, он не имеет никакого сходства с тем, как люди думают, учатся и понимают или как в онтологиях представляют знания.

Самые распространенные новейшие методы ИИ используют биоподобные нейронные сетевые архитектуры наряду с оптимизацией и статистическими подходами. Помимо того, что они являются субсимвольными, эти методы работают «снизу вверх» от данных, таких как текст и изображения. Взаимодействие с текстом и изображениями, подобными этому, сильно отличается от гораздо более широкого, биологически подобного опыта взаимодействия с миром. Хотя есть некоторые интеллектуальные системы, такие как самоуправляемые автомобили, которые имеют ограниченный диапазон таких взаимодействий, а обучение таких устройств в процессе работы представляется рискованным. Пример этого см. в [26].

К настоящему времени ИИ ещё не овладел более широкими формами обучения и понимания, которые происходят из реального опыта. Некоторые считают, что такое обучение на основе реального опыта требует начинать создание системы с когнитивного ядра, а затем последовательно разрабатывать более сложные когнитивные модели [7]. Социальный аспект реального, воплощённого опыта включает в себя изучение общих знаний от других интеллектуальных агентов, а также их информационные продукты, такие как текст, данные и физические действия. В то время как приобретение знаний о доменах и методов рассуждений домена продолжают улучшаться, оказалось, что очень сложно «закодировать» на машинах или реализовать обучение снизу вверх без каких-либо базовых знаний. Некоторая автоматизация необходима для решения основных проблем 
узких мест, которые могут помочь со здравым смыслом и рассуждениями, например, с ежедневными выводами, которые делают люди [1].

Методы МО являются в основном статистическими, поэтому включение неопределённости в онтологии было бы полезно при использовании МО в онтологиях. Ниже приведены два метода интеграции вероятности с семантикой.

- Статистическое реляционное обучение. Общий ИИ должен иметь дело как с реляционной структурой, так и с неопределённостью. Конкретное напрвление работы, посвящённое комбинации вероятностных моделей с логикой описания, известна как вероятностная семантика [27].

- Вероятностная мягкая логика (Probabilistic Soft Logic - PSL) - это система MO для разработки вероятностных моделей. PSL использует логические правила первого порядка как язык шаблонов для графических моделей над случайными величинами с мягкими значениями истинности из интервала $[0,1]$. Основополагающая математическая структура поддерживает чрезвычайно эффективную задачу непрерывной оптимизации вывода, которая может быть эффективно решена. PSL включает в себя способность логически обосновывать как атрибуты сущности, так и отношения между сущностями, а также онтологические ограничения. На практике с использованием PSL получены новые результаты во многих областях, охватывающих НЛП, анализ социальных сетей и компьютерное зрение. С помощью PSL проблемы с извлечением больших графов знаний с миллионами случайных величин могут быть решены на порядок быстрее, чем позволяют существующие подходы [5].

В дополнение к неопределённостям логического вывода есть много областей, разработка которых необходима для достижения того, чтобы ИИ стал универсальным и «здравым» (надёжным). На рисунке 3 показаны эти компоненты и их взаимодействие друг с другом.

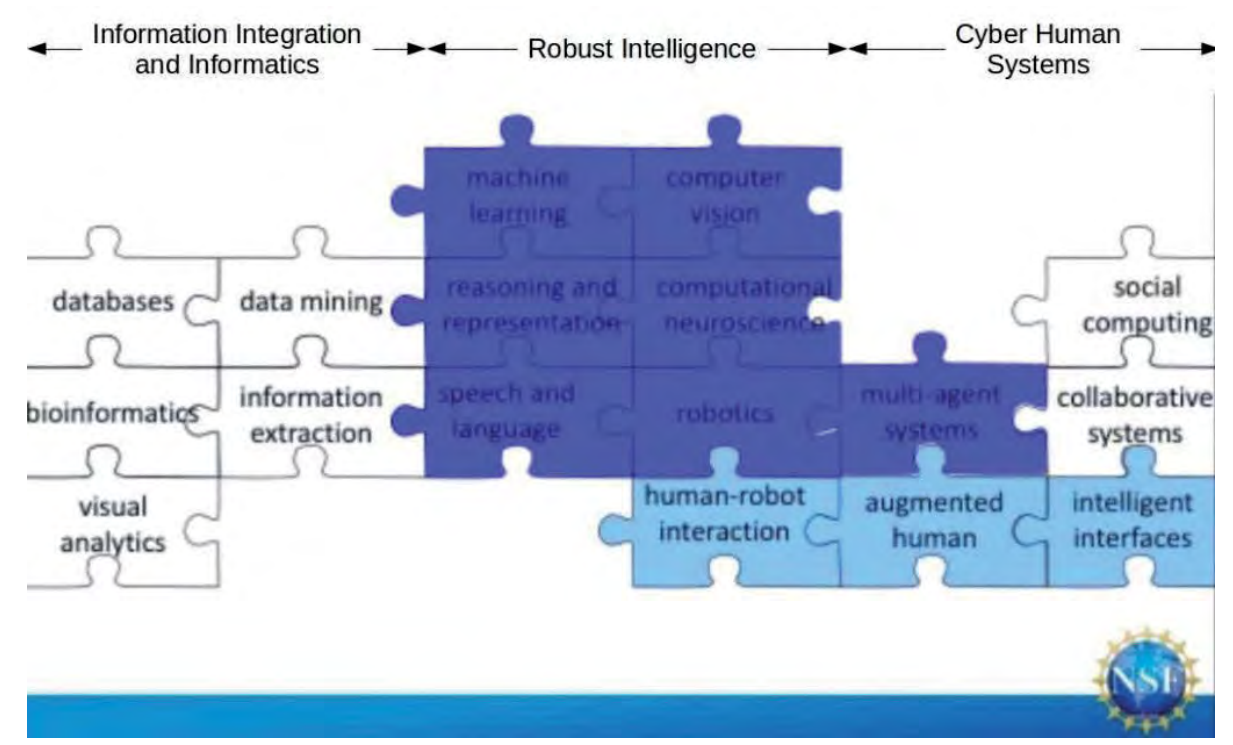

Рисунок 3 - Компоненты ИИ и их взаимодействие [1]

\section{4 Возможности и проблемы}

Разработано множество методов, которые используют обучение, логический вывод и исследования. Этот раздел представляет собой попытку показать некоторые проблемы и возможности взаимоотношений между обучением, рассуждениями и онтологиями.

\section{1 Прогнозы на основе биологической организации}

Клетка, наряду с системами тела, обычно моделируется с использованием уровней организации, каждая из которых может быть представлена с помощью онтологии - от молекулярного и биохимического уровня до клеточного и тканевого уровня, до органов и уровня систем органов и до уровня биосферы. Биологическую организацию часто называют «иерархией», но она не является иерархией в онтологическом смысле. Функционирование уровней организации, т.е. связь между уровнями, является формой рассуждения, называемой «биологическим выводом». Примером может служить карта генотипа-фенотипа [28]. МО, основанное на онтологиях, таких как ручная кураторская онтология гена (Gene Ontology - GO) [29], является основным методом биологи- 
ческого вывода из генов в их белковые продукты. Это МО использует обширные данные измерений сетевых связей между генами и их белковыми продуктами. GO структурирована для определения этих сетевых отношений с помощью трёх доменов GO: биологический процесс, клеточный компонент и молекулярная функция [12].

Биологический вывод использует знания о совместном выражении генов и взаимодействиях вида белокбелок, чтобы создать сеть сродства генов на основе данных. Процесс выравнивания определяет, какие термины данных являются новыми и какие повторяют существующие знания в GO. В совокупности это знание может быть сведено к онтологической иерархии и согласовано с GO, предлагая имена для новых терминов, основанных на данных. Большинство, около 60\%, отношений, найденных при деривации данных для клеточных компонентов, уже находятся в сотовой компоненте GO, но только около $25 \%$ полученных терминов для биологического процесса и молекулярной функции уже были найдены в GO, что указывает на то, что многие потенциально полезные знания могут быть обнаружены с использованием таких методов [12,30].

Не в каждой ПрО достигнуто единство мнений в выборе базовых онтологий, как в BioMedical. Аналогичным образом, не каждая ПрО имеет единый подход к уровням организации. Таким образом, возможность достижения успеха в применении онтологий в других ПрО - это открытая исследовательская проблема.

\section{2 Идентификация контекста}

Некоторые слова имеют много разных интерпретаций, которые сильно зависят от контекста [18]. Например, в контексте финансовой дискуссии, касающейся акций, термин «медведь», скорее всего, относится к отношениям инвесторов или фондовому рынку, а не к типам млекопитающих. Контекст имеет основополагающее значение для интерпретации, однако трудно формализовать понятие контекста. Возможно, лучшая позиция заключается в том, чтобы согласиться с Пэтом Хейсом [31] в том, что нет единого понятия контекста. По своей сути контекст включает понятия о том, что некоторые предложения, скажем, «есть много медведей на фондовом рынке», можно интерпретировать, что они истинны или ложны в контексте. Утверждение: «На фондовом рынке много белых медведей», скорее всего, ложно из-за контекста. То, что мы понимаем под контекстом, может зависеть от самого контекста. Важную работу в этой области провели Джон МакКарти и Пэт Хейс в их оценке ситуации [31], а также Баррилл, Перри и Девлин, которые разработали теорию ситуации [32], позднее формализованную как онтологию, выраженную на языке онтологии (OWL) [33,34]. Теория ситуаций очень популярна во многих областях, особенно в военных и бизнес-областях.

Хотя ситуационная теория во многих случаях является эффективной формализацией контекста, она не полностью определяет понятие контекста. Задача состоит в том, чтобы разработать эффективное формальное понятие контекста, которое может использоваться, чтобы устранить интерпретацию слов в человеческом дискурсе и потенциально привести к тому, что называется «пониманием». И оценка ситуации, и теория ситуации поддерживают процессы рассуждений. Теория ситуаций особенно универсальна в этом отношении, что позволяет использовать многие формы рассуждений [35].

\section{3 «Когнитивные леса»}

МО прошло долгий путь с момента определения Артуром Самуэлем в 1959 году как подраздела компьютерной науки, который даёт «компьютерам возможность учиться, не будучи явно запрограммированными» [36]. В этом смысле МО стало намного более выполнимым, с появлением всё более и более богатых наборов данных. Однако, как отмечено в разделе 3, мы всё ещё далеки от достижения МО без какого-либо явного программирования. Современные рабочие процессы МО часто включают рутинные задачи для: оценки проблем, исследования и предварительной обработки данных и обучения модели, а затем тестирования и развёртывания, которые все контролируются людьми. Тем не менее, некоторые применения МО стали более познавательными, контекстуальными и целостными, отходя от традиционных подходов к обучению «снизу-вверх». Для достижения этих функций требуется более интеллектуальная обработка и дополнительные знания.

Например, знания необходимы для обработки двусмысленности слов, таких как «реп», которые имеют несколько смыслов. Один смысл «реп» - это инструмент для записи, но также небольшое ограждение для удобства удержания детей или животных в зависимости от контекста. Но контекст здесь не является статистическим. Понимание предложения со словом «реп» в нём часто требует знания о реальном мире. Один из способов обеспечить такое знание - начать со «стартового» или «зачаточного» знания, которое можно расширить, применяя методы ИИ, такие как МО. Пример зачаточного знания для интеллектуального процесса был проиллюстрирован в системе NELL для одной важной когнитивной задачи: чтение [7]. Входы в NELL включают:

- первоначальную онтологию, определяющую сотни категорий (например, человек, спортивная команда, фрукты, эмоции) и отношения (например, играет в команде (спортсмен, спортивная команда), играет на инструменте (музыкант, инструмент)), о котором должна была прочитать NELL;

- 10 или 15 экземпляров зародышей знаний в каждой категории или отношении. 
Формирование начальных знаний в этом случае упрощалось за счёт уточнения тематики чтения NELL, например, чтение о спорте или музыке.

Более общий вопрос - это онтологическая основа достаточных знаний, необходимых автономному, интеллектуальному агенту, который наблюдает и действует в среде направленным образом для достижения целей. Это остаётся нерешённым вопросом. В настоящее время нет единой архитектуры, техники или инструмента для разработки интеллектуального агента даже для относительно простых информационных агентов, например, предусмотренных в первоначальной попытке реализации DAML [37]. Однако подходы изобилуют такими дисциплинами, как когнитивное развитие, когнитивная наука, робототехника развития и ИИ. Например, SOAR $[38,39,40]$ и ACT-R [41], а также BDI-архитектуры [42,43,44].

Но в подобных агентных системах трудно использовать такие вещи, как разнообразная пространственновременная информация, включая количественные и качественные оценки в рамках одного аналитического контекста за подходящий период времени. Тем не менее, в рамках аналитического процесса понимания ситуации, люди легко интегрируют как количественные, так и качественные оценки информации, чтобы прийти к выводам, и это происходит раньше чем люди, например, учатся читать. То есть, формирование начальных знаний для чего-то типа NELL, похоже, является частью процесса человеческого развития. Как это происходит? Представляется разумным предположить, что необходима некоторая степень врождённой структуры для развития когнитивной системы и соответствующих знаний для некоторых общих вещей как части опыта агента. Такое когнитивное развитие, особенно в контексте общего ИИ, иногда обсуждается в терминах ранних «когнитивных лесов» с основным набором когнитивных способностей, обеспечивающих временную структуру, позволяющую организовывать более общие знания и обучение во время прогрессивного развития в более богатую систему познавательных навыков.

В когнитивной науке знание воспринимается как основной результат процесса понимания: взаимодействуя с окружающей средой, интеллектуальные агенты способны интерпретировать и представлять факты об окружающем мире, соответствующим образом действуя, чтобы сохранять себя и преследовать конкретные цели $[45,46]$. Представление знаний является необходимым шагом для общения, но знание может быть представлено надлежащим образом только в той мере, в какой явления реального мира ранее были представлены людям, а именно через когнитивные структуры. Такие «когнитивные леса» могут быть определены как стартовый набор: тип динамического строительного блока.

К сожалению, в настоящее время в большинстве доменов нет принятых стартовых наборов, а также отсутствует общая теория относительно того, что такое стартовый набор или каковы первые узнаваемые компоненты знания и рассуждения. Например, гипотеза когнитивной лингвистики [47] предполагает, что, вероятно, общий человеческий опыт с миром прост и ограничен. Учитывая это, основная часть понимания основана на восприятии и действии. Эта основная семантика представлена в том, что некоторые называют «схемами изображений», которые действуют как метафорические рамки и когнитивные строительные блоки. Кандидаты на то, какие схемы изображений могут включать такие знакомые понятия онтологического основания, как: объекты, процесс и отношения часть-целое, движение, полный-пустой, контейнер, блокировка, поверхность, путь, связь, коллекция, слияние, масштабирование и выживание [48]. Некоторые работы по разработке шаблонов онтологий и справочные онтологии использовали эти понятия, такие как работа по сдерживанию, движению и пути.

Это ставит ряд проблем.

- К Каковы кандидаты на набор знаний, которые могли бы обеспечить адекватные когнитивные леса? Различные возможности обсуждались выше.

- В рамках «когнитивных лесов» интеллектуальный агент должен представлять соответствующие знания, чтобы они были доступны и пригодны для достижения цели агента. Как получаются мета-знания об обучении?

- В рамках «когнитивных лесов» интеллектуальные агенты нуждаются в механизмах контроля, чтобы найти соответствующие части знаний в конкретных контекстах. Как это учат и какие знания участвуют?

- Как агент может распознавать существующие шаблоны и объекты, даже с частичным и/или шумным вводом?

- Как агент может определить, к каким существующим категориям принадлежит шаблон, и насколько он соответствует этим категориям?

- Предсказание ближайшего будущего является важным требованием для понимания ситуации агентами. Даже если некоторый образ был распознан, как его можно проецировать в будущее?

- Как можно изучать и классифицировать новые модели и сущности?

- Как агент может выбрать соответствующую информацию на уровне ввода, а также во время обучения и познания?

- Как агент может изучать новые навыки, как умственные, так и физические? 


\section{4 Согласование онтологий}

В настоящее время нет единого мнения об онтологиях для обработки разнородной информации в эпоху Big Data. Несмотря на многочисленные усилия по созданию онтологий ПрО, словари и онтологии различных источников данных обычно не совместимы. Общие методы для объединения и согласования онтологий включают такие средства, как PROMPT, алгоритм полуавтоматического слияния и согласования онтологий [49]. Но, как было отмечено на онтологических саммитах и связанных с ними сессиях, есть проблемы в согласовании онтологий с различными предположениями и концепциями [50,51]. Известна работа по интеграции онтологий, в рамках которой с успехом были разработаны алгоритмы и эвристики, позволившие сделать такие вычисления выполнимыми [52]. Однако, как отмечено в [53], эффективное использование онтологических формализмов (т.е. правил и аксиом) как часть процесса интеграции остаётся открытым вопросом.

Одна из проблем, затрудняющих процесс интеграции онтологии, заключается в том, что в рамках процесса нам необходимо понять взаимосвязь между структурами знаний (классами и свойствами) и данными экземпляра в целевых онтологиях. Существующие методы согласования онтологий и выравнивания очень ограничены. Они находят сходства, эквивалентности и отношения подчинения между двумя (или более) онтологиями, которые должны, по крайней мере, быть синтаксически и схематически интегрированы, иметь схожий объём и контекст и быть более выразительными, чем OWL. Напротив, семантическая интеграция между существующими областями, например, гидрология, её онтологии и схемы, требует также перевода между онтологическими языками и более строгой спецификации семантики в каждой онтологии. В настоящее время это можно сделать только путём ручной интеграции онтологий, но использование подходящей эталонной онтологии может помочь автоматизировать это [51]. Кроме того, интеграция должна обладать способностью использовать семантику онтологии для моделирования взаимосвязей между интегрируемыми онтологиями и создания последовательной и единообразно интегрированной или согласованной онтологии.

Другим постоянным источником проблем при сопоставлении частей онтологий является то, что онтология спроектирована на основе определённых фоновых знаний (аксиоматизированных или нет) с определённой целью и в определённом контексте (явном или неявном). Контекст онтологии может включать в себя опыт онтологов, которые разработали онтологию, их предпочтение конкретным онтологиям верхнего уровня, предметным словарям, шаблонам проектирования онтологии или исходным данным для моделирования. Они могут не быть частью спецификации онтологии, что делает их недоступными для инструментов объединения или сопоставлений объектов/отношений. Это отсутствие фоновых знаний и контекста может привести к двусмысленности [54].

Одним из примеров попытки решения проблем, связанных с онтологией, является Проект по оценке согласованности онтологий (Ontology Alignment Evaluation Initiative - OAEI), который проводит конкурсы на согласование онтологий [55]. В ОАЕІ предлагается сложный набор онтологий и источников данных, которые должны быть сопоставлены. Например, предложено сопоставить анатомию взрослой мыши (2744 класса) с тезаурусом Национального института рака (3304 класса), который описывает анатомию человека. Как и в других случаях используются систематические серии эталонных тестов. Задача серии тестов заключалась в определении областей, в которых каждый алгоритм согласования является сильным или слабым.

\section{5 Идентификация и выделение графра знаний}

Реальность больших данных позволяет запрашивать массивные хранилища потенциально взаимосвязанных фактов. К сожалению, как отмечалось на предыдущих онтологических саммитах [56,50], представление этой информации в форме, позволяющей сделать её полезным знанием, является серьёзной проблемой.

Одна интересная задача - преобразовать исходный материал (обычно естественный текст) в форму графа знаний. Граф знаний - это структура, где сущности - это узлы графа, категории - это метки слов, связанные с каждым узлом, а отношения - это направленные рёбра между узлами. Таким образом, граф знаний представляет собой одну упрощённую версию онтологии и нечто менее формальное, чем концептуальные графики J.Sowa. Такие усилия по созданию даже этой простой структуры требуют разрешения идентификации объектов и связей с сущностями. Существует степень неопределённости и шума в таких отношениях, а также необходимость вывести недостающую информацию и определить, какие факты-кандидаты должны быть включены в граф знаний как часть процесса идентификации. Один из подходов заключается в следующем:

1) назначить рейтинги уверенности в необходимости выделения каждому факту-кандидату,

2) идентифицировать связанные сущности,

3) установить онтологические ограничения.

Этот подход основан на вероятностной мягкой логике (PSL), недавно введённого метода вероятностного моделирования, который легко масштабируется до миллионов фактов, как было продемонстрировано с выделением из проекта NELL более одного миллиона фактов-кандидатов и 70 тысяч онтологических отношений [57]. 
Тем не менее, остаётся открытой проблема для расширения итеративных методов извлечения знаний и обучения таких систем, как NELL, для систем, которые способны изучать, сохранять и использовать знания на протяжении всей жизни. Богатые возможности для продвижения в ИИ, как отмечает Silver, «лежат в локусе машинного обучения и представления знаний; в частности, методы консолидации знаний обеспечат понимание того, как наилучшим образом представлять знания для использования в будущем обучении и логическом выводе» [58].

\section{6 Процессы}

Должно быть очевидно, что обучение, логический вывод и онтологии происходят в составе более крупных процессов, где они и отношения между ними образуют этапы. J.Sowa предложил: «Для интеллектуальных систем когнитивный цикл более фундаментален, чем любое конкретное описание или алгоритм». Затем он пришел к выводу, что «интегрируя восприятие, обучение, рассуждение и действие, цикл может активизировать исследования и разработки ИИ» [59]. Несколько презентаций саммита подчеркнули важность процессов, которые в основном были в форме своего рода «петель обратной связи» $[9,12,19,21,60]$. Будем называть такие петли, как «когнитивные циклы». «Существует много примеров когнитивных циклов, в которых происходят обучение, рассуждение и онтологии. Процесс научных открытий является примером с длинной историей [21]. Многие виды деятельности можно рассматривать как циклы принятия решений, в которых каждая итерация улучшает понимание и осознание путём поиска новых знаний, а также путём отказа от некоторых предыдущих знаний $[19,24]$.

В прошлом одна итерация когнитивного цикла, такого как научный процесс, могла занять десятилетия. Сегодня когнитивные циклы происходят быстрее, гораздо больше данных необходимо обрабатывать, а данные стали сложнее. Изучение, логический вывод и онтологии, а также отношения между ними, которые являются предметом этого саммита, могут играть важную роль в когнитивных циклах. На предыдущих саммитах также рассматривались когнитивные циклы, включая работу с огромным количеством данных [61], которые поступают от большого числа сенсоров [62] и требуют большого количества шагов, которые должны взаимодействовать [63].

Сочетание обучения, логического вывода и онтологий в рамках когнитивных циклов имеет потенциальные преимущества, но обычно это практически не используется. В той степени, в которой такие процессы автоматизированы вообще, они, как правило, являются узкоспециальными и неформальными. Чтобы автоматизировать процесс научных открытий, необходимо использовать НЛП для извлечения материалов и методов, используемых в экспериментах [64], т.е. процесса экспериментальных действий и генерируемых научных гипотез [21]. Ещё одним требованием является выявление источников, т.е. происхождение фактов и знаний. Задача состоит в том, чтобы разработать необходимые онтологии, стандартизировать их, сформулировать передовые практики и убедить сообщества использовать их. В некоторых случаях, таких как PROV-O для происхождения [65] и OPMW [66] для рабочего процесса научных открытий, онтологии были стандартизированы, но другие требования когнитивного цикла менее продвинуты. Ни один из них не используется широко, и лучшие практики только начинают появляться [19,21].

Существует много способов, которыми обучение, логический вывод и онтологии можно объединить друг с другом в когнитивный цикл. Обучение и логический вывод являются основополагающими для каждого из этапов когнитивного цикла, а также перехода от одного шага к другому. Онтологии могут использоваться для организации этих вычислительных процессов. Такие методы могут использоваться для обработки поступающей информации, которые используют онтологию для организации данных, а также помогают в разработке этих онтологий. Онтологии могут предоставить дополнительную пользу, помогая сделать данные и их обработку более понятными. Онтологии могут служить основой для объяснения результатов, что помогает повысить доверие к системе. Обучение и логический вывод можно также использовать на метауровне для оптимизации когнитивного цикла, выявления и устранения проблем. В совокупности онтологии, обучение и логический вывод могут быть использованы для обеспечения того, чтобы компоненты системы взаимодействовали друг с другом в соответствии с планами разработчиков.

\section{7 Критика и фрлуктуации}

Хорошо известно, что история исследований ИИ является историей подъёмов и спадов [67]. Подразделы ИИ, а также область ИИ в целом, существенно изменялись, и существует риск того, что это произойдет снова. Область онтологии также не защищена от этого риска. Хотя некоторые ПрО, такие как биомедицина, используют онтологии в значительной степени и очень успешно, в других ПрО, таких как производство, не было достигнуто значительных успехов в использовании онтологий, несмотря на то, что их было разработано множество [68]. ИИ настолько быстро развивается во многих секторах экономики, что сообщество начинает сомне- 
ваться в том, стоит ли инвестировать в технологии ИИ [69]. Одним из возможных способов смягчения риска является серьёзное отношение к критике и реакция на неё, а не игнорирование или высмеивание замечаний, как это зачастую было в прошлом [70].

Область Big Data в целом критиковалась в течение нескольких лет [71], и МО подвергается некоторым из тех же критических замечаний. Обучение и логический вывод улучшают связь между онтологиями, но не могут полностью решить проблему согласования, они могут только помочь.

1) Понимание результатов по-прежнему имеет важное значение. Действительно, сильный ИИ, думающий как человек, в конечном счёте, сталкивается с проблемой представления и использования знаний, доступных людям. Этот вопрос широко обсуждался в разделах 4.2, 4.3 и 4.6, и многие презентации на саммите подчёркивали важность этого вопроса и предлагали методы борьбы с ним путём укрепления связей между МО и онтологиями $[14,15,19,21]$.

2) Результаты МО чаще всего представляют собой совокупность корреляций. Одна общая ошибка - это предположение, что корреляция автоматически подразумевает причинность. Понимание результатов МО в человеческих терминах может помочь устранить, по крайней мере, наиболее неправдоподобные примеры вывода причинности из корреляции.

3) Часто бывает недостаточно согласованности и совместимости данных, используемых в приложениях с большими данными. Совместимость была темой онтологического Саммита 2016 [63] и обсуждалась в разделах 2.4, 4.4 и 4.6.

4) Общей критикой приложений Big Data является то, что задаваемые вопросы часто слишком неточны. Другими словами, существует разрыв между запросами, выполняемыми с данными, и интерпретацией результатов. Точность - одна из целей онтологий. Улучшение отношений между онтологиями и МО позволило бы обеспечить последовательность и понятность интерпретаций.

Проблема разочарования в области онтологии в некоторых ПрО, таких как производство, потенциально может быть решена путём создания «основной» инициативы в каждой ПрО, аналогичной открытой биологической онтологии (Open Biological Ontolog - OBO) [72], которая стала очень успешной в биомедицинском сообществе. Тем не менее, это открытая проблема: можно ли повторить успех ОВО в других ПрО [68].

\section{5 Заключение}

На онтологическом саммите 2017 года был рассмотрен широкий круг вопросов, возможностей, проблем и будущих перспектив взаимосвязи между обучением, логическим выводом и онтологиями в контексте ИИ. Мы узнали, что можно выявить полезные знания о биологической организации, используя МO и GO, но вопрос, возможно ли достижение этого в других областях, остаётся открытым. Мы обсудили проблему определения понятия контекста, которая остаётся нерешённой, хотя в некоторых областях был достигнут определённый прогресс. Мы отметили, что ИИ вообще и МО в частности не возможны без каких-либо фоновых знаний. Мы рассмотрели множество проблем в развитии «когнитивных лесов» для таких знаний. Мы пересмотрели вопрос об интероперабельности, который был рассмотрен на прошлогоднем саммите, но по конкретной проблеме согласования онтологии, и решили, что это серьёзная открытая проблема, хотя по ней имеется прогресс. Мы рассмотрели проблему извлечения графов знаний из исходного материала для использования в МО и в логическом выводе и отметили возможности для достижения ИИ с использованием методов итеративного извлечения знаний и обучения для общих приложений ИИ. Мы исследовали процессы, которые организуют приложения обучения, логического вывода и онтологий, а также отношения между ними; мы предложили возможности для решения многих важных проблем, связанных с общими приложениями ИИ. Мы показали, как можно в некоторой степени учесть некоторые из недавних критических замечаний о MO и Big Data путём укрепления связей между обучением, логическим выводом и онтологиями. Мы также рассмотрели вопрос о том, как можно решить проблему принятия методов онтологического проектирования в областях, в которых не достигнуто заметных успехов.

\section{6 Благодарности}

В настоящем коммюнике упомянуты некоторые коммерческие программные системы. Такое упоминание не предполагает рекомендации или одобрения со стороны Национального института стандартов и технологий (NIST) или организаций авторов или составителей этого коммюнике; а это также не означает, что упомянутые продукты являются наилучшими. Кроме того, любые мнения, выводы или рекомендации, выраженные в этом материале, являются мнениями авторов и не обязательно отражают взгляды NIST или любых других поддерживающих правительственных или корпоративных организаций США. 


\section{Список источников}

[1] H. Wactlar. Robust intelligence research at NSF. In Ontology Summit 2017: AI, Learning, Reasoning, and Ontologies, 16 May 2017. s3.amazonaws.com/ontologforum/OntologySummit2017/Symposium/SymposiumRecording--HowardWactlar 20170516.mp4.

[2] R. McMillan. AI has arrived and that really worries the world's brightest minds, 16 January 2015. www.wired.com/2015/01/ai-arrived-reallyworries-worlds-brightest-minds/.

[3] D. Silver, Q. Yang, and L. Li. Lifelong machine learning systems: Beyond learning algorithms. In AAAI Spring Symposium: Lifelong Machine Learning, volume 13, 2013. Ontology Summit 2017

[4] P. Buitelaar. Ontology learning - some advances. In Ontology Summit 2017: AI, Learning, Reasoning, and Ontologies, 1 March 2017. s3.amazonaws.com/ontologforum/OntologySummit2017/Overview/OntologyLearning-SomeAdvances--PaulBuitelaar 20170301.pdf.

[5] L. Getoor. Combining statistics and semantics to turn data into knowledge. In Ontology Summit 2017: AI, Learning, Reasoning, and Ontologies, 19 April 2017. s3.amazonaws.com/ontologforum/OntologySummit2017/TrackC/Turning-Data-into-Knowledge--LiseGetoor 20170419.pdf.

[6] G. Berg-Cross. Track A Summary Report: Using automation and machine learning to extract knowledge and improve ontologies. In Ontology Summit 2017: AI, Learning, Reasoning, and Ontologies, 15 May 2017.

s3.amazonaws.com/ontologforum/OntologySummit2017/Symposium/Symposium-TrackA-Synthesis-GaryBergCross20170515.pdf, s3.amazonaws.com/ontologforum/OntologySummit2017/Symposium/SymposiumRecording--GaryBergCross 20170515.mp4.

[7] E. Hruschka. Never-Ending Learning approach for populating and extending an ontology. In Ontology Summit 2017: AI, Learning, Reasoning, and Ontologies, 8 March 2017. s3.amazonaws.com/ontologforum/OntologySummit2017/TrackA/OverviewOfNELLEstevamHruschka 20170308.pdf.

[8] F. Corcoglioniti. Frame-based ontology population from text with PIKES. In Ontology Summit 2017: AI, Learning, Reasoning, and Ontologies, 5 April 2017.

s3.amazonaws.com/ontologforum/OntologySummit2017/TrackA/OntologyFromTextWithPIKESFrancescoCorcoglioniti20170405.pdf.

[9] J. Aasman. Cognitive probability graphs need an ontology. In Ontology Summit 2017: AI, Learning, Reasoning, and Ontologies, 22 March 2017. s3.amazonaws.com/ontologforum/OntologySummit2017/TrackC/ProbabilityGraphsAndOntologies--JansAasman 20170322.pptx.

[10] E. Pafilis. EXTRACT 2.0: interactive extraction of environmental and biomedical contextual information. In Ontology Summit 2017: AI, Learning, Reasoning, and Ontologies, 5 April 2017. s3.amazonaws.com/ontologforum/OntologySummit2017/TrackA/EXTRACTEvangelosPafilis 20170405.pdf.

[11] P. Buitelaar, P. Cimiano, and B. Magnini, editors. Ontology Learning from Text: Methods, Evaluation and Applications. IOS Press, 2005.

[12] M. Yu. Inferring the hierarchical structure and function of a cell from millions of biological measurements. In Ontology Summit 2017: AI, Learning, Reasoning, and Ontologies, 5 April 2017. s3.amazonaws.com/ontologforum/OntologySummit2017/TrackA/InferringCellHierarchyMichaelYu 20170405.pdf.

[13] M. Bennett and A. Westerinen. Track B Summary Report: Using background knowledge to improve machine learning results. In Ontology Summit 2017: AI, Learning, Reasoning, and Ontologies, 15 May 2017.

s3.amazonaws.com/ontologforum/OntologySummit2017/Symposium/Symposium-TrackBSummary-MGB-20171115.pdf,

s3.amazonaws.com/ontologforum/OntologySummit2017/Symposium/SymposiumRecording--MikeBennett 20170515.mp4.

[14] V. Presutti. Semantic Web machine reading with FRED. In Ontology Summit 2017: AI, Learning, Reasoning, and Ontologies, 8 March 2017. content.iospress.com/articles/semantic-web/sw240.

[15] S. Davidson. The Investigator's Toolkit deriving immediate, actionable insights from unstructured data. In Ontology Summit 2017: AI, Learning, Reasoning, and Ontologies, 15 March 2017. s3.amazonaws.com/ontologforum/OntologySummit2017/TrackB/TrackB-PSONIFY Ontology-Summit-Davidson-20170315.pdf.

[16] C. Falk. The Meaning-Based Machine Learning Project. In Ontology Summit 2017: AI, Learning, Reasoning, and Ontologies, 12 April 2017. s3.amazonaws.com/ontologforum/OntologySummit2017/TrackB/TrackB-2 Meaning-Based+Machine+Learning-CourtneyFalk 20170412.pdf.

[17] T. Erekhinskaya. Converting text into FIBO-aligned semantic triples. In Ontology Summit 2017: AI, Learning, Reasoning, and Ontologies, 12 April 2017. s3.amazonaws.com/ontologforum/OntologySummit2017/TrackB/TrackB-2 Erekhinskaya Tatiana--20170412.pdf.

[18] B. Bell and E. Kendall. Leveraging FIBO with semantic analysis to perform on-boarding, KYC and CDD. In Ontology Summit 2017: AI, Learning, Reasoning, and Ontologies, 12 April 2017. s3.amazonaws.com/ontologforum/OntologySummit2017/TrackB/TrackB-2Ontology Summit 2017Cognitive+Computing+brings+FIBO+Alive 20170412-Bell.pdf.

[19] K. Baclawski. Toward combining ontologies and machine learning for improving decision making, 15 March 2017. Track B Session 1.

[20] D. Fritzsche and R.D. Sriram. Track C Summary Report: Ontologies and Reasoning. In Ontology Summit 2017: AI, Learning, Reasoning, and Ontologies, 15 May 2017. s3.amazonaws.com/ontologforum/OntologySummit2017/Symposium/SymposiumRecording--RamDSriram.mp4.

[21] Y. Gil. Reasoning about scientific knowledge with workflow constraints: Towards automated discovery from data repositories, April 2017. s3.amazonaws.com/ontologforum/OntologySummit2017/TrackC/Gil-AutomatedDiscovery-OntologySummit2017 20170419.pdf.

[22] E. Kuksa. Reasoning with ontologies in Ontohub. In Ontology Summit 2017: AI, Learning, Reasoning, and Ontologies, 22 March 2017. s3.amazonaws.com/ontologforum/OntologySummit2017/TrackC/ReasoningInOntohub--EugenKuksa 20170322.pdf.

[23] S. Rugaber. Applications of ontologies to biologically inspired design. In Ontology Summit 2017: AI, Learning, Reasoning, and Ontologies, 19 April 2017. s3.amazonaws.com/ontologforum/OntologySummit2017/TrackC/BiologicallyInspiredDesign--SpencerRugaber 20170419.pdf.

[24] P. Hitzler. On the roles of logical axiomatizations for ontologies. In Ontology Summit 2017: AI, Learning, Reasoning, and Ontologies, 22 March 2017. s3.amazonaws.com/ontologforum/OntologySummit2017/TrackC/RolesOfLogicForOntologies--PascalHitzler 20170322.pdf.

[25] P. Koehn. Statistical machine translation. Cambridge University Press, Cambridge, UK, 2010.

[26] K. Baclawski, K. Gross, E.S. Chan, D. Gawlick, A. Ghoneimy, and Z.H. Liu. Self-adaptive dynamic decision making processes. In IEEE Conference on Cognitive and Computational Aspects of Situation Management, 2017. cogsima2017.ieeecogsima.org/files/2016/01/1570328101Baclawski.pdf.

[27] S. Pileggi. Probabilistic semantics. Procedia Computer Science, 80:1834-1845, 2016.

[28] The genotype-phenotype distinction, 2017. en.wikipedia.org/wiki/Genotype-phenotype distinction.

[29] The Gene Ontology Consortium. The Gene Ontology project in 2008. Nucleic Acids Research, 36 (Database issue):D440-4, January 2008. doi:10.1093/nar/gkm883. PMC 2238979Freely accessible. PMID 17984083.

[30] T. Hahmann, S. Stephen, and B. Broderic. Domain reference ontologies vs. domain ontologies: What's the difference? Lessons from the water domain, 10 November 2016. s3.amazonaws.com/ontologforum/DomainVocabularies/DomainReferenceOntologies--Hahmann-StephenBroderic 20161110.pdf.

[31] P. Hayes. Contexts in context. In AAAI Fall Symposium, 1997.

[32] K. Devlin. Logic and Information. Cambridge University Press, Cambridge, U.K., 1991. 
[33] K. Baclawski, M. Malczewski, M. Kokar, J. Letkowski, and C. Matheus. Formalization of situation awareness. In Eleventh OOPSLA Workshop on Behavioral Semantics, pages 1-15, Seattle,WA, November 42002.

[34] M. Kokar, C. Matheus, and K. Baclawski. Ontology-based situation awareness. Information Fusion, 10(1):83-98, 2009.

[35] K. Baclawski, E.S. Chan, D. Gawlick, A. Ghoneimy, K. Gross, Z.H. Liu, and X. Zhang. Framework for ontology-driven decision making. Applied Ontology, 2017. to appear.

[36] A. Samuel. Some studies in machine learning using the game of checkers. IBM Journal of Research and Development, pages 535-554, 1959.

[37] DARPA Agent Markup Language homepage, 2006. www.daml.org/.

[38] J. Laird, A. Newell, and P. Rosenbloom. SOAR: an architecture for general intelligence. Artificial Intelligence, 33(1):1-64, September 1987.

[39] A. Newell. Unified Theories of Cognition. Harvard University Press, Cambridge, MA, USA, 1990.

[40] R. Wray and J. Laird. An architectural approach to ensuring consistency in hierarchical execution. J. Artificial Intelligence Research, 19:355398, 2003.

[41] J. Anderson and C. Lebiere. The atomic components of thought. Lawrence Erlbaum Associates, Hillsdale, NJ, 1998. ISBN 0-8058-2817-6.

[42] M. Georgeff and A. Lansky. Procedural knowledge. Technical Report Technical Report 411, Artificial Intelligence Center, SRI International, 1987. Ontology Summit 2017

[43] M. Huber. A BDI-theoretic mobile agent architecture. In AGENTS '99. Proceedings of the Third Annual Conference on Autonomous Agents, pages 236-243, Seattle, WA, USA, May 1999. ACM.

[44] A. Rao and M. Georgeff. Modeling rational agents within a BDI-architecture. In R. Fikes and E. Sandewall, editors, Proceedings of Knowledge Representation and Reasoning (KR\&R-91), pages 473-484. Morgan Kaufmann Publishers, San Mateo, CA, 1991.

[45] U. Neisser. From direct perception to conceptual structure. In Concepts and Conceptual development, pages 11-24. Cambridge University Press, Cambridge, 1987.

[46] L. Albertazzi. Which semantics? In L. Albertazzi, editor, Meaning and cognition. A multidisciplinary approach, pages 1-24. Benjamins Publishing Company, Amsterdam, 2000.

[47] M. Johnson. The Body in the Mind: The Bodily Basis of Meaning, Imagination, and Reason. The University of Chicago Press, 1990.

[48] A. Oltramari. An introduction to hybrid semantics: the role of cognition in semantic resources. In Modeling, Learning, and Processing of Text Technological Data Structures, pages 97-109. Springer Berlin Heidelberg, 2011.

[49] N. Noy and M. Musen. PROMPT: algorithm and tool for automated intelligence. In AAAI-2000, Austin, TX, 2000.

[50] Ontology Summit 2012: Ontology for Big Systems, 2012. ontologforum.org.org/index.php/OntologySummit2012.

[51] S. Stephen and T. Hahmann. Semantic alignment of the Groundwater Markup Language with the Reference Hydro Ontology HyFO, 2016. ontologforum.s3.amazonaws.com/OntologySummit2016/2016-02-25ＧeoSciences/Stephen-Ontology\%20Summit\%20Talk\%202016-03-31 v5.pdf.

[52] J. Euzenat. An api for ontology alignment. In International Semantic Web Conference. Springer Berlin Heidelberg, 2004.

[53] O. Udrea, L. Getoor, and R. Miller. Leveraging data and structure in ontology integration. In Proc. 2007 ACM SIGMOD International Conference on Management of Data. ACM, 2007.

[54] P. Shvaiko and J. Euzenat. Ten challenges for ontology matching. In Proc. 7th International Conference on Ontologies, Databases, and Applications of Semantics (ODBASE), pages 1163-1181, Monterey, Mexico, 2008. Springer-Verlag. Lecture Notes in Computer Science; On the Move to Meaningful Internet Systems: OTM 2008.

[55] Ontology Alignment Evaluation Initiative, 2016. oaei.ontologymatching.org/2016/.

[56] Ontology Summit 2009: Toward Ontology-based Standards, 2009. ontologforum.org.org/index.php/OntologySummit2009.

[57] J. Pujara, H. Miao, L. Getoor, and W. Cohen. Knowledge graph identification. Lecture Notes in Computer Science, 8218:542-557, 2013. repository.cmu.edu/cgi/viewcontent.cgi?article=1021\&context=machine learning.

[58] D. Silver. Lifelong machine learning and reasoning. In I. Robinson, J. Webber, and E. Eifrem, editors, Graph Databases, pages 75-78. O'Reilly Media, 2013.

[59] J. Sowa. Why has AI failed? And how can it succeed?, May 10 2015. www.jfsowa.com/talks/micai.pdf.

[60] A. Oltramari. From machines that learn to machines that know: the role of ontologies in machine intelligence. In Ontology Summit 2017: AI, Learning, Reasoning, and Ontologies, 8 March 2017.

s3.amazonaws.com/ontologforum/OntologySummit2017/TrackA/RolesOfOntologiesInMachineIntelligence-lessandroOltramari20170308.pdf.

[61] Ontology Summit 2014: Big Data and Semantic Web Meet Applied Ontology, 2014. ontologforum.org.org/index.php/OntologySummit2014.

[62] Ontology Summit 2015: Internet of Things: Toward Smart Networked Systems and Societies, 2015. ontologforum.org.org/index.php/OntologySummit2015.

[63] Ontology Summit 2016: Framing the Conversation: Ontologies within Semantic Interoperability Ecosystems, 2016. ontologforum.org/index.php/OntologySummit2016.

[64] K. Baclawski, R. Futrelle, N. Fridman, and M. Pescitelli. Database techniques for biological materials \& methods. In First Int. Conf. Intell. Sys. Molecular Biology, pages 21-28, 1993.

[65] PROV Ontology (PROV-O), April 30 2013. http://www.w3.org/TR/2013/REC-prov-o-20130430/.

[66] Open Provenance Model for Workflows (OPMW), December 22 2014. http://www.opmw.org/model/OPMW 20141222.

[67] History of Artificial Intelligence, 2017. en.wikipedia.org/wiki/History of artificialOntology Summit 2017 intelligence.

[68] B. Smith. The Industry Foundry Ontology. In Ontology Summit 2017: AI, Learning, Reasoning, and Ontologies, 15 May 2017. s3.amazonaws.com/ontologforum/OntologySummit2017/Symposium/SymposiumRecording--BarrySmith 20170515.mp4.

[69] Artificial intelligence software is booming. But why now?, 19 September 2016. www.nytimes.com/2016/09/19/technology/artificialintelligence-software-is-booming-but-why-now.html.

[70] Critiques of Artificial Intelligence, 2017. en.wikipedia.org/wiki/History of artificial intelligence\#Critiques from across campus.

[71] G. Marcus and E. Davis. Eight (no, nine!) problems with Big Data. NY Times, 6 April 2014. www.nytimes.com/2014/04/07/opinion/eight-nonine-problems-with-big-data.html.

[72] Open Biological Ontologies website, 2003. obo.sourceforge.net. 


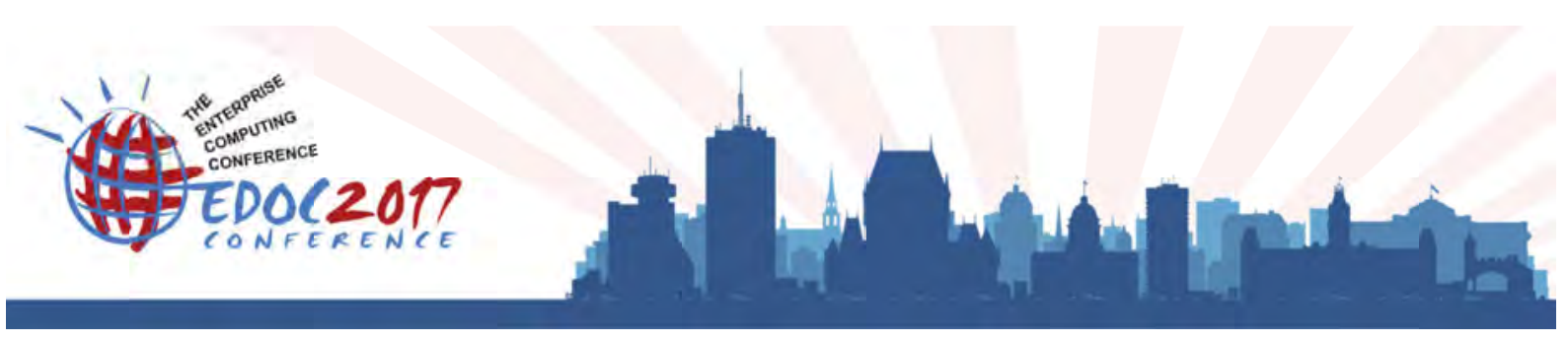

\title{
9th International Workshop \\ on Vocabularies, Ontologies and Rules for the Enterprise \\ Co-located with the 21th IEEE International EDOC Conference - The Enterprise Computing Conference 10th-13th October, 2017, Quebéc-City, Canada
}

\author{
About \\ the evaluation of such systems and approaches. \\ oriented architectures) that enable collaboration between heterogeneous enterprise systems. \\ rules in the enterprise system development lifecycle. \\ - Vocabularies and Ontologies for the enterprise:

\section{Topics} \\ - Ontology-driven representation of products, services, functionalities, design, processes; \\ - Ontologies for the know-how; \\ - Ontologies for corporate knowledge; \\ - U pper-level ontologies in the enterprise; \\ - Adaptation of ontologies for companies and organizations; \\ - Conceptual and Enterprise Modeling: \\ - Ontological F oundations for Conceptual M odeling and M eta-modeling \\ - Ontology D esign and maintenance for Conceptual M odel Integration; \\ - Ontology-Based Conceptual Modeling Tools and Environments \\ - Ontologies and Enterprise Architecture Modeling \\ - Ontologies and Business Process M odeling \\ - Ontologies and Goal-Based M odeling \\ - Ontologies and Business Rules \\ - Enterprise Semantic Interoperability: \\ - Web services, Semantic services \\ - Composition and Modularity \\ - Merging, M apping and Alignment \\ - Ontology Language Interoperability \\ - Open linked data publishing and exploitation \\ - Knowledge management: \\ - Ontology and ontological methodologies in knowledge management and integration; \\ - Ontology engineering, learning and maintenance; \\ - Ontology integration and alignment; \\ - Ontology effectiveness and evaluation; \\ - Case studies and Evaluation: \\ - Practical issues in using ontologies in enterprises \\ - Real cases of successful/unsuccessful use of ontology in business \\ - From legacy systems to the new ontology-driven systems
}

The VORTE series of workshops is devoted to the topics of vocabularies, ontologies and rules in the context of enterprise systems. Examples of topics covered by VORTE include the development and adaptation of foundational, business and domain ontologies for the enterprise, the use of ontologies and rules in all aspects of enterprise modeling such as business process management and services, the enhancement of rules and services with formal semantics, and

From the enterprise system development perspective, research topics are focused on relations of process modeling and execution languages with business ontologies and rules, and on how business ontologies and rules used in enterprise models are further propagated into technologies (e.g., linked data and semantic web) and architectures (e.g., service-

The workshop also welcomes experience reports and empirical studies that are reporting on the use of ontologies and

http://edoc2017.ca/vorte-2017/ 


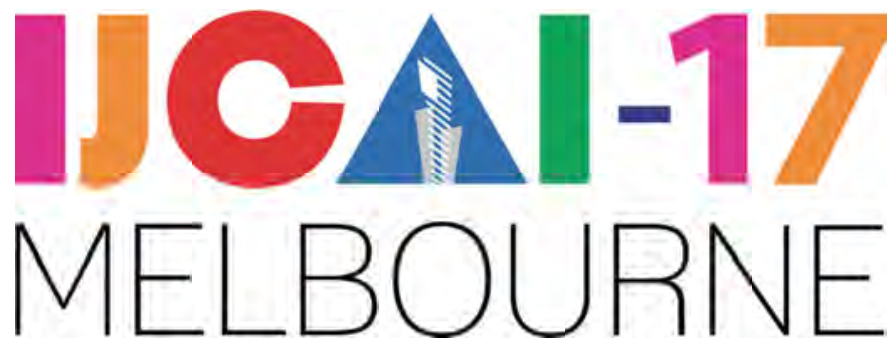

IJCAI is the International Joint Conference on Artificial Intelligence, the main international gathering of researchers in AI. IJCAI were held biennially in odd-numbered years since 1969. Starting with 2016, IJCAI will be held annually. IJCAI is sponsored jointly by IJCAI and the national AI societie(s) of the host nation(s). The 26th International Joint Conference on Artificial Intelligence will be held in Melbourne, Australia in August 2017.

\section{JCAI 2017 Accepted Workshops}

- Fourth International Workshop on Theory and Applications of Formal Argumentation

- Fifth International Workshop on Graph Structures for Knowledge Representation and Reasoning

- $\quad$ Third International Workshop on Social Influence Analysis (Soclnf 2017)

- Workshop on Computer Games

- Workshop on Language Sense on Computer

- Workshop on Learning in the Presence of Class Imbalance and Concept Drift

- Impedance Matching in Cognitive Partnerships

- Evaluating General-Purpose Al (EGPAl2017)

- The 3rd workshop on Algorithmic Game Theory

- Workshop on Linked Democracy: Artificial Intelligence for Democratic Innovation

- Workshop on Cognitive Knowledge Acquisition and Applications (Cognitum 2017)

- Third Workshop on Conflict Resolution in Decision Making (COREDEMA 2017)

- Explainable Al

- Workshop on Al Applications in E-Commerce

- Workshop on Linguistic and Cognitive Approaches to Dialogue Agents (LaCATODA)

- Workshop on Knowledge Discovery in Healthcare II: Towards Learning Healthcare Systems

- Ninth International Workshop on Modelling and Reasoning in Context (MRC 2017)

- Workshop on Artificial Intelligence and Constraint Programming

http://ijcai-17.org/index.html

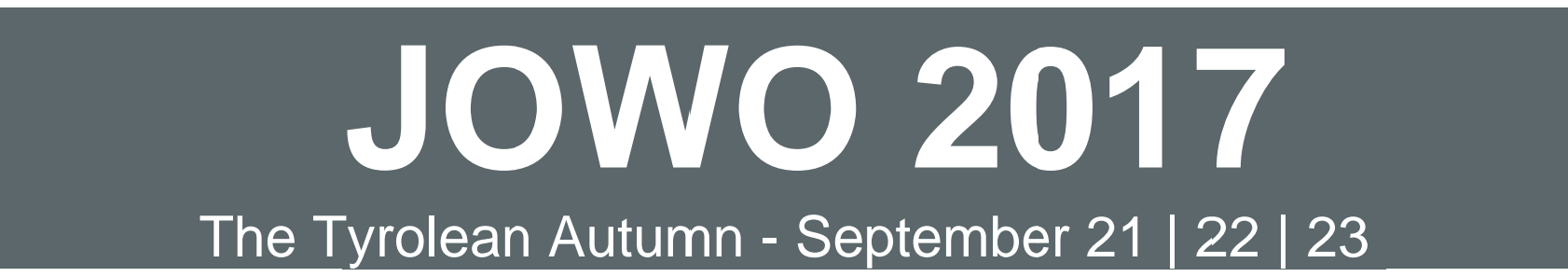

The Joint Ontology Workshops take place at the Free University of Bozen-Bolzano, Italy.

This third edition of the Joint Ontology Workshops (JOWO-17) includes the following workshops.

CREOL:

DAO:

DEW:

EPINON:

FOMI:

FOUST II:

ISD3:

ODLS:

SHAPES 4.0:

WINKS:

Contextual Representation of Objects and Events in Language

Data meets Applied Ontology

Ontology Evaluation \& Debugging

Epistemology in Ontologies

Formal Ontology Meets Industry

2nd Workshop on Foundational Ontology

The 3rd Image Schema Day

Ontologies and Data in Life Sciences

Workshop on Interaction-Based Knowledge Sharing

In addition to workshops, JOWO will be hosting joint panel sessions and keynotes, as well as meetings of IAOA Special Interest Groups and Technical Committees.

$$
\text { http://www.iaoa.org/jowo/2017/ }
$$




\section{Индекс 29151}

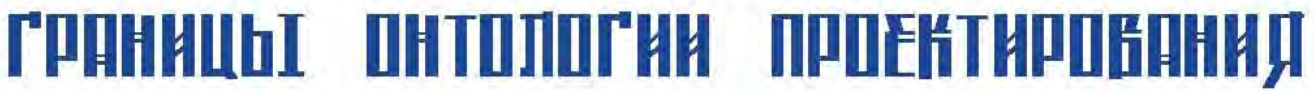

О границах научно-образовательной дисциплины

«Онтология проектирования» читайте в 1-ом номере журнала за 2017 г.

статью профессора Самарского университета Боргеста Н.М.

Онтолоеия проектирования рассматривает сущностные вопросы проектирования в различных предметных областях (ПрО): исследует объект проектирования, создавая его семантические модели, исследует субъект проектирования, как актора в различных ролях, и саму быстроменяющуюся среду проектирования, влияющую на формирование критериев объекта и субъекта.

\section{ФОКУС ИССЛЕДОВАНИЙ}

Инжинирине онтолоеий - разработка и применение методов и инструментов, позволяющих создавать онтологии, с акцентом на онтологии ПрО в проектировании любых объектов и систем. Ожидаемый результат - разработка языков, методов и средств формализации.

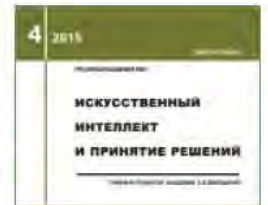

Методы и технолоеии принятия решений - методы, технологии и системы поддержки принятия решений в условиях субъективной и объективной неопределённости и многокритериальности.

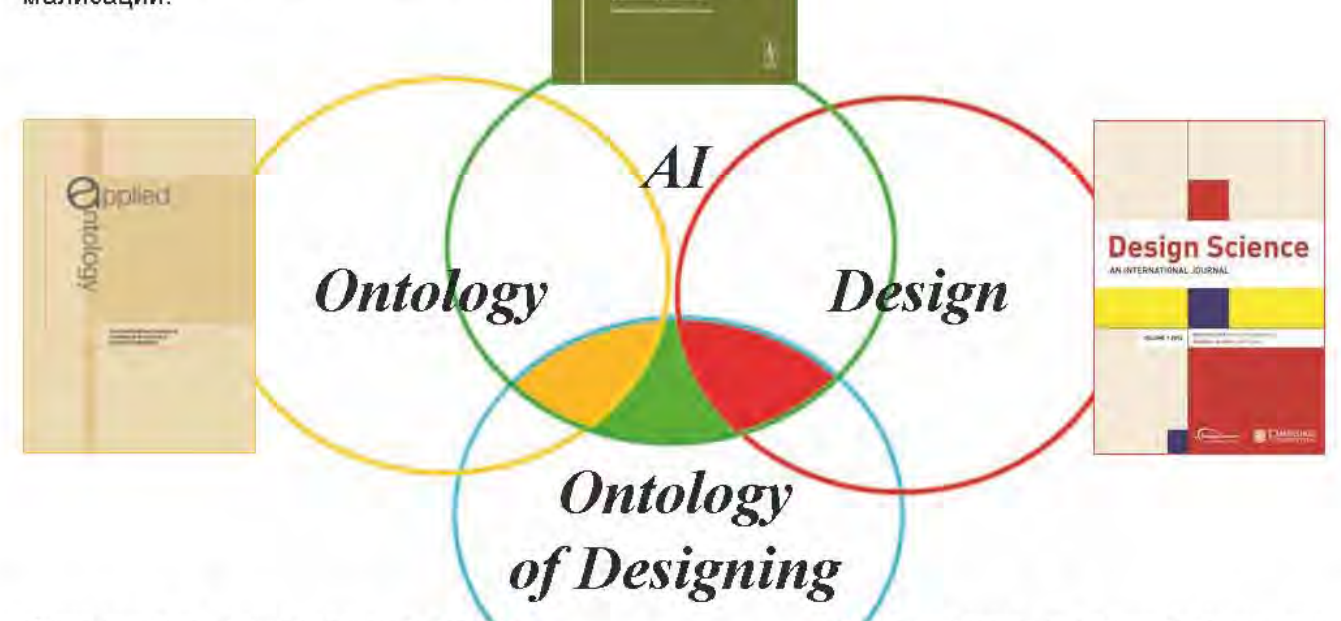

Прикладные онтолоеии проектирования - разработка онтологий в различных ПрО. Особое внимание сущностным вопросам, постановкам проектных и оптимизационных задач (проектная область, проектные переменные, критерии...), чёткой формулировке используемых понятий, критериев, методов. Ожидаемый резульmam - формализация Про и решаемых в ней задач на семантическом, концептуальном уровне $c$ позиций технических и естественнонаучных дисциплин.

философские и психолоаические аспекты проектирования - кон-

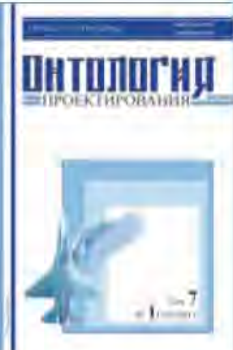
структивные решения, которые можно будет конвертировать в формализмы как для разрабатываемых интеллектуальных помощников, так и при решении конкретных проектных и управленческих задач. Ожидаемый резульmam - адаптация гуманитарных знаний (философских, психологических, лингвистических) к их формализации в задачах проектирования, выработка конструктивных методов моделирования будущего.

\section{Продолжается подписка}

на журнал «Онтология проектирования» на 2017 год. Подробности на сайте журнала.

Ontologists and designers of all countries and subject areas, join us!

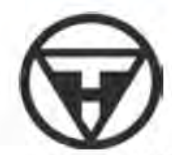

Издательство "Новая техника" - Publisher «New Engineering» Ltd Россия, 443010, Самара, ул.Фрунзе 145 - 145, Frunze Str., Samara, 443010, Russia 